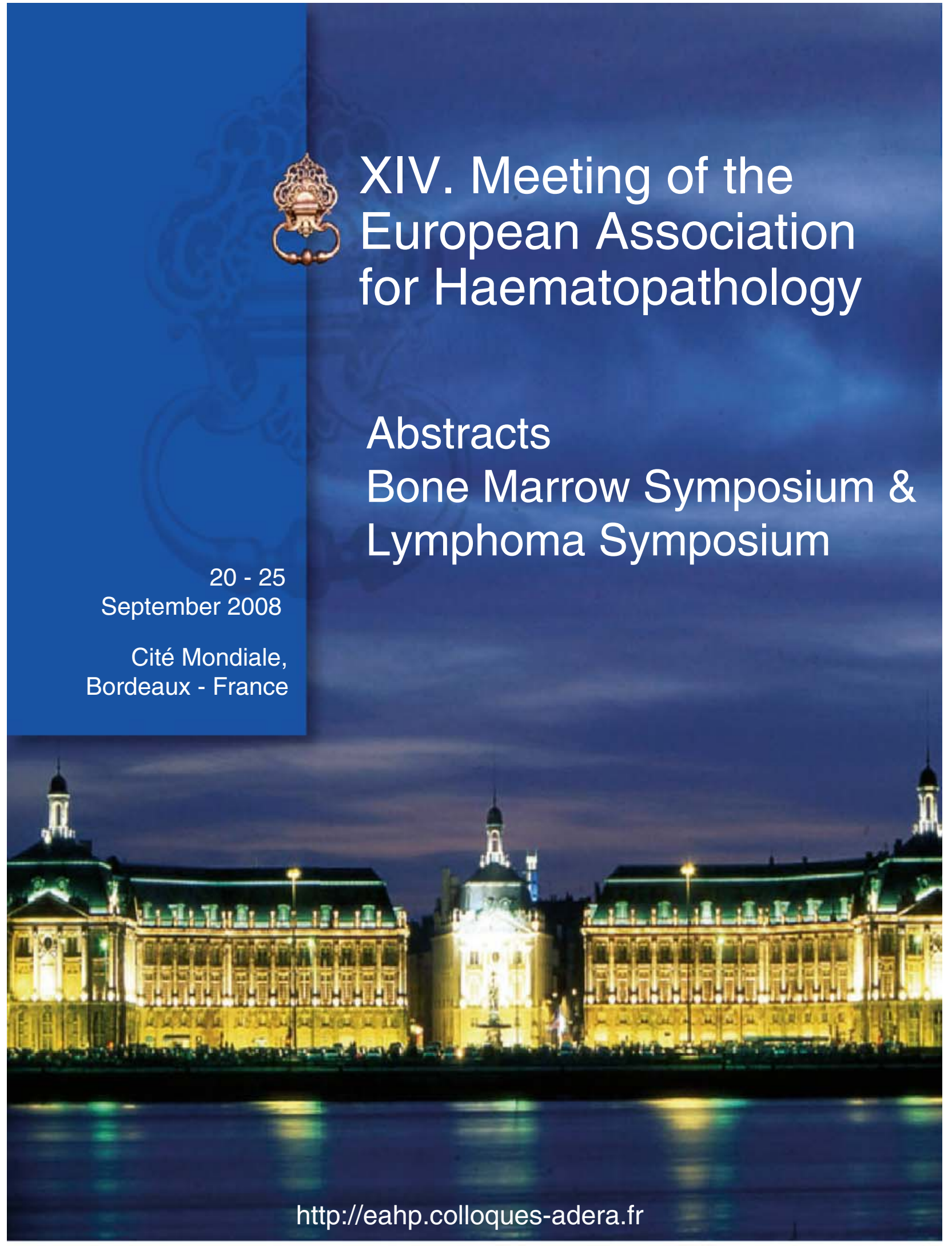




\section{International Scientific Committee}

- Andreas Chott

- Antoine de Mascarel

- Ahmet Dogan

- Martin Hansmann

- Daniel Jones

- Hans Kreipe

- Steven Pals

- Stefano Pileri

- Miguel Piris

- Anna Porwit

- Leticia Quintanilla-Fend

\section{Local Organizing Committee}

- Antoine de Mascarel

- Marie Parrens

- Jean-Philippe Merlio

- Béatrice Vergier

- Isabelle Soubeyran

\section{Lymphoma Workshop Panel:}

- Philip Kluin

- Daphne de Jong

- Antoine de Mascarel

- Eric Hsi

- Yaso Natkunam

- German Ott

- Stefano Pileri

- Leticia Quintanilla-Fend

\section{Bone Marrow Workshop Panel}

- Vito Franco

- Hans Kreipe

- Attilio Orazi

- Anna Porwit

- Jon van der Walt 


\section{Bone Marrow Symposium 21 September 2008}

\section{BMS 1}

\section{DIFFERENTIAL EXPRESSION OF BMI-1 IN VARIOUS TYPES OF MYELODYSPLASTIC SYNDROME (MDS) AND WITH IMPLICATION OF ITS ROLE IN PATHOGENESIS}

\author{
Kai Zhang, MD and J. Shi, MD and Fan Lin MD, Ph.D.
}

Department of Laboratory Medicine and Pathology, Geisinger Medical Center, Danville, PA

Background: The Bmi-1 gene is a putative oncogene and is essential and indispensable for the regulation of self-renewal of normal and leukemic stem cells. In this study, we investigated Bmi-1 expression on bone marrow core biopsy samples from various types of MDS to assess its possible role in MDS.

Design: Using an EnVision-HRP detection kit (Dako), we evaluated the expression of Bmi-1 in 60 cases of bone marrow biopsy specimens using monoclonal anti-Bmi-1 antibody. These included 10 cases for each of following 6 groups according the updated WHO classification: Group 1(G1)- benign bone marrow, Group 2 (G2)-MDS-RA (refractory anemia), Group 3(G3)-MDS-RARS (RA ringed sideroblast), Group 4 (G4)MDS-EB1 (excess blast type-1), Group 5(G5) MDS-EB2 (excess type-2), and Group 6 (G6) MDS-AML (acute myeloid leukemia). The staining intensity was graded as weak, moderate, or strong. The types and percentage of Bmi-1 positive cells (erythroid precursors and immature myeloid cells) was counted manually. Cytoplasmic and/or nuclear staining for Bmi-1 was regarded a positive result.

Results: In G1 (benign marrow), megakaryocytes and occasional primarily large erythroblasts and early myeloid precursors showed mild to moderate cytoplasmic and/or nuclear staining. Approximately 5\% of small rubricytes and metarubricytes were weakly positive. In G2 (MDSRA)and G3 (MDS-RARS), Bmi-1+ rubricytes and metarubricytes were significantly increased up to $20 \%$; and $80 \%$ respectively, which were associated with increased staining intensity with strong nuclear staining. In G4, G5 and G6, the majority of recognizable erythroid precursors and megakayrocytes were found to be strongly positive for Bmi- 1 . There was a significant difference among these three groups in terms of Bmi-1+ immature myeloid cells or blasts, with the number of Bmi-1+ cells of $8 \%$ in G4, 15\% in G5, and over 30\% in G6, respectively. Importantly, all Bmi$1+$ positive cells showed dominantly strong nuclear staining signal instead of the cytoplasmic staining.

Conclusion: Increased abnormal hematopoietic populations of either erythroid precursors or immature myeloid /blasts among various types of MDS are correlated with 1) the overexpression of Bmi-1 and 2) the switching of Bmi-1 expression from the cytoplasmic staining to nuclear staining, which suggests that Bmi-1 may play an important role in the pathogenesis of MDS.

\section{BMS 2}

\section{Evolution of the Accelerated and Blastic Phases of Chronic Myeloid Leukemia: Molecular, Cytogenetic, Flowcytometric and Electron Microscopic Studies}

Amira M Khorshed, MD.; Amira H Soliman, MD.; Nahed Abdel Wahab, MD.; Sherine Ibrahim, MD.; Khaled A. Emara, MD.; Reham Rashed, Tarek Mansour, MD.*; Naglaa Kholousy, MD.**; Fayek M. Ghaleb, MD. ***

Departments of Clinical Pathology and Cancer Biology*, National Cancer Institute, Cairo University and Immunogenetic Department, National Research Center** and Clinical Pathology Department, Ophthalmology Research Institute***

Background: Chronic Myeloid Leukemia is a clonal disease that results from an acquired genetic change in a pluripotential hemopoietic stem cell. Molecular abnormalities and mutations usually accompany the accelerated and blastic crisis phases of CML.

Aim of Work: This study was conducted to explore the possible ultrastructural, molecular cytogenetic, apoptotic and morphological abnormalities that may contribute to the progression of chronic phase to accelerated and blastic crisis phases in CML patients, for focusing on high risk patients to justify accurate lines of treatment.

Subjects and Methods: The study included thirty CML patients newly diagnosed and under treatment presenting to the Medical Oncology department of the National Cancer Institute, Cairo University, and ten age-matched subjects as a control group. CD95 (FAS) and P53 were studied by flowcytometry, BCR/ABL gene was studied at the cytogenetic and molecular level by RT PCR and ultrastructural apoptotic changes were studied by EM in PB samples. Also FISH was performed on few selected cases where conventional cytogenetics was not informative.

Results: Mean level of P53\% was highly increased in the accelerated $\& /$ or blastic crisis phase and the chronic phase compared with controls $(p=0.04)$. Mean level of CD95\% expression was higher when measured on the whole cell population in the (accelerated \&/or blastic crisis) compared with chronic phase and controls $(\mathrm{p}=0.14)$. By selecting CD34+ve cells, lower levels of CD95\% expression were found in the (accelerated \&/or blastic crisis phase) compared with the levels expressed on the whole cell population in the same phase. Cases were divided according to follow up into Group 1: 16/30 $(53.3 \%)$ chronic phase cases that remained chronic during treatment. 
Group 2: $12 / 30(40 \%)$ chronic phase cases that developed an accelerated or blastic crisis during treatment then returned to the chronic phase. Group 3: 2/30 (6.6\%) chronic phase cases that developed an accelerated or blastic crisis phase then died. Mean P53 $\%$ levels showed no statistically significant difference between the groups of CML ( $p=0.85)$. Higher levels of CD95\% in CD34+ve cells were expressed in Group 1 compared with Group 2 and Group 3 and were statistically insignificant $(p=0.85)$. Mean levels of CD95\% expression were higher when measured on the whole cell population in Group 2 than Group 1 and Group $3(p=0.45)$. Mean level of P53 \% in the treated cases was higher compared to newly diagnosed cases (before treatment) showing a statistically significant difference $(\mathrm{p}=0.01)$. Higher mean levels of CD95\% on whole cell population, and on CD34+ve selected cells were detected after treatment $(p=0.30, p=0.83)$. The mean levels of $\mathrm{P} 53 \%$ and CD95\% were higher in BCR/ABL fusion gene positive cases than BCR/ABL fusion gene negative cases but didn't reach significant levels respectively $(\mathrm{p}=0.21, \mathrm{p}=0.62)$.

Conclusion: a) P53\% and CD95\% levels expression in the accelerated and blastic crisis phases of CML patients were higher than those in the chronic phase. b) comparative studies for the apoptotic markers with cytogenetic analysis and RT PCR techniques revealed higher levels of $\mathrm{P} 53$ and $\mathrm{CD} 95$ in $\mathrm{BCR} / \mathrm{ABL}$ positive cases than BCR/ABL negative cases c) P53 and CD95 levels were higher in treated cases than newly diagnosed cases.

\section{BMS 3}

\section{SCLEROSING MESENTERITIS: A RARE MIMIC OF MYELOPROLIFERATIVE DISORDERS.}

\section{George Fedoriw, Nelofar Shafi}

Department of Pathology and Laboratory Medicine, Yale University School of Medicine, New Haven, CT, USA

Department of Pathology and Laboratory Medicine, Veterans Affairs Connecticut Healthcare System, West Haven, CT, USA

Systemic and localized non-neoplastic conditions can significantly influence the bone marrow microenvironment, inducing changes mimicking clonal neoplastic disorders. In most cases, the diagnostic dilemma is quickly resolved and disease etiology elucidated as presenting symptoms, laboratory and histopathologic features appropriately guide the clinical course. The purpose of this report is to present the laboratory and clinical data of a patient with sclerosing mesenteritis, a rare non-neoplastic fibrosing condition associated with features of myeloproliferative disorders. Our patient is a 70 year old man who presented with shortness of breath, drenching night sweats, lower extremity edema, abdominal discomfort, and recent $20 \mathrm{lb}$ weight loss. His past medical history was significant for prostatic adenocarcinoma treated with surgery and radiation, umbilical hernia repair, and hypertension. Clinical exam revealed hepatosplenomegaly and no palpable lymphadenopathy. Laboratory findings showed a microcytic anemia with peripheral blood smear examination revealing significant anisopoikilocytosis characterized by dacrocytosis and polychormasia, and marked leukocytosis. CT scan confirmed hepatosplenomegaly and showed nonspecific changes including "streaking" of the mesenteric soft tissue; changes initially suspected to be involvement by lymphoproliferative disorder. Subsequent bone marrow evaluation revealed a markedly hypercellular marrow with panmyelosis, left shifted myeloid maturation, and reticulin fibrosis.
No increase in myeloid blasts was present morphologically or flow cytometrically. Cytogenetic and molecular analysis demonstrated a normal karyotype and no evidence of $\mathrm{BCR} / \mathrm{ABL}$ translocation or JAK-2 mutation. Progressive radiologic changes of the intra-abdominal soft tissue prompted a mesenteric biopsy with histologically confirmed features of sclerosing mesenteritis. Treatment with prednisone and tamoxifen was initiated with rapid and significant improvement in symptoms, laboratory values and bone marrow histology. This report highlights the clinical, laboratory and bone marrow histopathologic changes in a patient with sclerosing mesenteritis presenting as a myeloproliferative disorder. The full range of available diagnostic modalities should be employed to establish the correct diagnosis and initiate timely treatment.

\section{$\underline{\text { BMS } 4}$}

\section{DISCORDANT IMMUNOPHENOTYPIC PROFILES IN ACUTE MYELOID LEUKEMIA INVOLVING BONE MARROW AND SKIN (LEUKEMIA CUTIS)}

Reena Sachdev, MD ${ }^{1}$, Tracy I. George, $\mathrm{MD}^{1}$, and *Uma N. Sundram, MD, $\mathrm{PhD}^{1,2}$

Departments of Pathology ${ }^{1}$ and Dermatology ${ }^{2}$, Stanford University Medical Center, Stanford, California, USA

Background: Leukemia cutis (LC) occurs in less than 5\% cases of acute leukemia, and its occurrence may constitute progression of disease. The parameters governing occurrence of LC are poorly understood.

Purpose: We investigated expression patterns of certain homing receptors via immunohistochemistry in skin lesions involved by acute myeloid leukemia (AML), to determine if skin homing plays a role in extramedullary involvement. Ten paired cases of skin and bone marrow biopsies from patients with LC and 15 bone marrow biopsies from patients without LC were studied with antibodies directed against CD34, cutaneous lymphocyte antigen (CLA), CD62-L (Lselectin), CD54 (ICAM-1), CD29 ( $\beta 1$ integrin subunit), and CD183. Results: CLA was expressed in all cases of leukemia (both marrow and skin involvement), whereas CD54 was consistently negative. CD34 was expressed in 5/10 biopsies of marrow infiltrates with LC and 3/10 biopsies of matching skin infiltrates; in marrows without LC, $8 / 15$ were positive. CD62-L was expressed in $4 / 10$ biopsies of marrow infiltrates with LC and 6/10 biopsies of matching skin infiltrates; in marrows without LC, only $2 / 15$ were positive. CD29 was expressed in $1 / 10$ biopsies of marrow infiltrates with $\mathrm{LC}$ and $4 / 10$ biopsies of matching skin infiltrates; in marrows without LC, only $1 / 15$ were positive. CD183 was expressed in $1 / 10$ biopsies of marrow infiltrates with LC and 4/10 biopsies of matching skin infiltrates; in marrows without LC, CD183 was entirely negative

Conclusions: Our results indicate that CLA and CD54 expression in bone marrow may not be necessary or sufficient for involvement of skin in AML. The gain of CD62-L, CD29, and CD183 expression in bone marrow and skin infiltrates in $\mathrm{LC}$, relative to bone marrow infiltrates of cases without LC, suggests a potential role for these markers in AML homing to skin. 


\section{BMS 5}

\section{CLINICAL CORRELATES AND PROGNOSTIC VALUE OF BONE MARROW HISTOLOGICAL PARAMETERS IN PRIMARY MYELODYSPLASTIC SYNDROMES}

* Emanuela Boveri, ${ }^{1}$ Matteo Giovanni Della Porta, ${ }^{2}$ Luca Malcovati, ${ }^{2}$ Alessandro Castello, ${ }^{1}$ Erica Travaglino, ${ }^{1}$ Barbara Burroni, ${ }^{1}$ Marco Lucioni, ${ }^{1}$ Marco Paulli, ${ }^{1}$ Cristiana Pascutto, ${ }^{2}$ Francesco Passamonti, ${ }^{2}$ Elisa Rumi, ${ }^{2}$ Luca Arcaini, ${ }^{2}$ Rosangela Invernizzi, ${ }^{3}$ Umberto Magrini, ${ }^{1}$ Mario Lazzarino, ${ }^{2}$ and Mario Cazzola. ${ }^{2}$

${ }^{1}$ Department of Surgical Pathology, ${ }^{2}$ Department of Hematology and ${ }^{3}$ Department of Internal Medicine and Medical Oncology, Fondazione IRCCS Policlinico San Matteo, University of Pavia, Italy.

Background: In myelodysplastic syndromes (MDS), bone marrow (BM) histology allows the evaluation of parameters, which are complementary to the informations obtained by marrow smear and karyotype.

Purpose of the study is to determine the clinical correlates and prognostic value of BM histological parameters in 301 consecutive primary MDS patients diagnosed according to WHO criteria between 2000 and 2006.

Results: By univariable statistical analysis, the following histological variables affect overall survival (OS) and leukemia-free survival (LFS): age-related cellularity (OS HR 1.008, $P=.005$; LFS HR 1.011, $P=.003$ ), percentage of CD34+ cells (OS HR 1.017, $P<.001$; LFS HR 1.028, $P<.001$ ), presence of BM CD34+ cell clusters (OS HR 2.87, $P<.001$; LFS HR 3.89, $P<.001$ ), grade 2-3 BM fibrosis (OS HR 4.39, $P<.001$; LFS HR 3.48, $P<.001$ ). In multivariable analysis, grade 2-3 BM fibrosis and $\mathrm{BM} \mathrm{CD} 34+$ cell clusters have an independent negative impact on OS $(P<.001$ and $P=.019$, respectively $)$ and LFS $(P<.001$ and $P=.004$, respectively). Grade $2-3 \mathrm{BM}$ fibrosis (17\% of cases) is associated with multilineage dysplasia $(P=.001)$, high transfusion need $(P<.001)$, poor risk cytogenetics $(P=.007)$. BM CD34+ cell clusters $(23 \%$ of patients $)$ is associated with WHO categories with excess of blasts $(P<.001)$ and poor risk cytogenetics $(P=.001)$. A hierarchical clustering analysis identifies 3 subsets of patients with distinct clinical features. One of these clusters mainly consists of patients with $\mathrm{BM}$ fibrosis, multilineage dysplasia, high transfusion need; these patients show lower OS and LFS ( $P=.001$ and $P<.001$, respectively). Within patients stratified according to WHO classification-based prognostic scoring system, BM fibrosis signifies a shift to a one-step more advanced risk group.

Conclusion: $\mathrm{BM}$ fibrosis and $\mathrm{CD} 34+$ cell clusters are histological parameters with a strong diagnostic and prognostic significance in MDS patients; BM fibrosis identifies a distinct subgroup of MDS with poor prognosis, and represents an independent prognostic factor that can be useful in clinical decision-making.

\section{BMS 6}

\section{JAK2V617 ACTIVATING MUTATION IN CHRONIC MYELOMONOCYTIC LEUKEMIA}

*Achille Pich, Ludovica Riera, Francesca Sismondi, Laura Godio, Laura Davico Bonino, Paola Francia di Celle.

Department of Biomedical Sciences and Human Oncology, Section of Pathology, University of Turin, Italy.
Background: Chronic myelomonocytic leukemia (CMML) has heterogeneous clinical and morphologic features, and is classified in the WHO myeloproliferative-myelodysplastic overlap category. The Janus Kinase 2 (JAK2) V617F mutation was detected in 40 to $94 \%$ of patients with myeloproliferative disorders, such as polycythemia vera, essential thrombocythemia and myelofibrosis with myeloid metaplasia, but only in a small number of patients with myelodysplastic/ myeloproliferative diseases or myelodysplastic syndromes.

Purpose: The purpose of this study was to search for JAK2 V617F mutations in CMML and investigate the association with clinical, hematological and bone marrow (BM) histological features.

Materials and Methods: Sixty-five consecutive patients (18 females and 47 males; mean age: 71 years) with newly diagnosed CMML from 2004 to 2007 were included in the study. JAK2 V617F mutation was assessed using direct sequencing of exon 14 or by allele-specific PCR from total peripheral blood or bone marrow samples. BM biopsies were stained with H\&E, Dominici, Perls, Gomori and immunostained for CD34, CD68PGM1, CD31, Factor VIII-RA, myeloperoxidase and glycophorin.

Results: JAK2 V617F mutation was identified in $5(7.7 \%)$ of 65 cases. Patients with JAK2 V617F mutation presented all with a marked splenomegaly and had a significantly higher haemoglobin level $(13.5 \mathrm{~g} / \mathrm{dL}$ vs $10.7 \mathrm{~g} / \mathrm{dL}, \mathrm{p}=0.01)$ and neutrophil count $(14.5 \times$ $10^{9} / \mathrm{L}$ vs $\left.6.8 \times 10^{9} / \mathrm{L}, \mathrm{p}=0.01\right)$ than patients without the mutation. BM biopsies of CMML with JAK2 V617F mutation showed in all cases marked myeloid hyperplasia, moderate to marked megakaryocytic hyperplasia, with occasionally clustered megakaryocytes, and mild to moderate reticulin fibrosis; three showed an increased number of dilated sinusoids.

Conclusions: Our results indicate that JAK2 V617F mutation can be detected in a small number of CMML and is associated with clinical and morphologic features of the myeloproliferative type of CMML. Therefore, this finding could help in a more appropriate classification of the disease.

\section{BMS 7}

\section{BONE MARROW ANGIOGENESIS IN HODGKIN'S LYMPHOMA}

${ }^{* 1}$ Tatjana Terzić, ${ }^{2}$ Vesna Čemerikić-Martinović, ${ }^{3}$ Maja PeruničićJovanović, ${ }^{3}$ Ljubomir Jaković, ${ }^{1}$ Gordana Basta-Jovanović

${ }^{1}$ Institute of Pathology, School of Medicine, University of Belgrade, Serbia; ${ }^{2}$ Histolab, Belgrade, Serbia; ${ }^{3}$ Institute of Hematology, Clinical Center of Serbia, Belgrade, Serbia

Background: Angiogenesis is essential step for tumor growth, invasion and progression. Bone marrow infiltration is present in $5-15 \%$ of patients with Hodgkin's lymphoma (HL). It is usually associated with prominent fibrosis and proliferation of blood vessels. Limited information exists about the significance of neoangiogenesis in HL.

Purpose of this study: was to analyse the microvessel density, imunohistochemicaly marked with monoclonal antibody CD34, in reactive bone marrow and bone marrow of patients with HL.

Results: We retrospectively analysed bone marrow biopsies of 43 patients with HL (26 male and 17 female, median age 44 years), 25 patients with bone marrow infiltration by $\mathrm{HL}$ and 18 patients without bone marrow infiltration. These bone marrow biopsies were compared with reactive bone marrow samples of 5 patients without any 
hematological malignancy (control group). Bone marrow biopsies were stained with hematoxylin\&eosin and reticulin fibers (GordonSweet). Angiogenesis was estimated by microvessel density (MVD) using standard immunohistochemical staining for CD34. We counted the number of vessels per high power field $(\times 500)$ in two the most vascularized areas. We found increase of microvessels density (MVD) in bone marrow patients with $\mathrm{HL}$, but without bone marrow lymphoma infiltration $(9,30 \pm 5,23)$, compared with control bone marrow $(3,50 \pm 0,71)$, but correlation was not statistically significant $(p>0.05)$. We observed that MVD inside the bone marrow lymphoma infiltrate $(17,63 \pm 9,27)$ in HL patients was higher than MVD in HL patients without bone marrow infiltration $(9,30 \pm 5,23)$. This correlation was statistically significant $(\mathrm{p}<0.05)$.

Conclusion: Our findings suggest that angiogenesis in bone marrow infiltration by HL was extensively increased. Therefore, the density of microvessels may provide basis for the therapeutic investigation of anti-angiogenic agents in the cases of HL with bone marrow infiltration.

\section{BMS 8}

\section{COMPARTMENTALIZATION OF CD20 B-CELL RECEPTOR INTO GLYCOSPHINGOLIPID-RICH MICRODOMAINS INDUCES THE COMMONLY OBSERVED "DOWNREGULATION" OF CD20 IN RITUXIMAB TREATED B-NHL}

\author{
C. Aumueller ${ }^{1}$, C. Meyer zum Bueschenfelde ${ }^{2}$, I.Koch $^{1}$, M. Perker ${ }^{3}$, \\ L. Quintanilla-Martinez ${ }^{4}$, F. Fend ${ }^{1,5}$ and M. Kremer ${ }^{1}$
}

${ }^{1}$ Institute of Pathology, Technical University, Munich, ${ }^{2}$ III Internal Department, Klinikum rechts der Isar, Technical University, Munich, ${ }^{3}$ Center for Hematology, Weilheim, Helmholtz-Research Center, Neuherberg, ${ }^{5}$ Institute of Pathology, University of Tuebingen, Germany

Background: Rituximab has shown significant efficacy in patients with B-cell NHL. Loss or downregulation of CD20 during rituximab therapy has been observed and held responsible for secondary resistance. However, systematic immunohistochemical and molecular studies examining this downregulation in a larger series of cases are lacking.

Aims: We performed a retrospective analysis of imunophenotypic changes in sequential biopsies of patients with a variety of B-cell NHL before, during and after rituximab therapy. Additonally we investigated the molecular mechanisms, responsible for the observed loss or downregulation in NHL cell lines.

Design: A total of 131 formalin-fixed lymph node and bone marrow biopsies of 87 pts. with B-NHL (28 FL, 37 MCL, 3 MZL, 14 DLBCL, 1 CLL) and three cell lines (NCEB-1, DB, MEC1) and primary cells from B-CLL were analyzed. Biopsies were taken between 0-16 months after initiation of Rituximab therapy. Protein and mRNA expression of CD20, PAX5, CD79a, CD3, CD5, CD23, CD38, FMC7, CD10, kappa, lambda were analyzed by IHC, FACS, Western Blot, and qRTPCR. IgH rearrangement analysis was performed in selected cases.

Results: Twenty-eight cases studied achieved CR without immunophenotypical or molecular evidence of lymphoma in the post-therapy samples. A complete downregulation of CD20 was observed in $23 / 28$ $(82 \%)$ of these cases
Fifty-five cases showed persistent disease or developed relapse. A total loss of CD20 expression was noticed in 6/55 (11\%), a partial downregulation in $17 / 55(31 \%)$ of the cases.

CD20 downregulation was clearly time-dependent, with the highest frequency within the first three months of Rituximab therapy. CD20 protein expression was considerably diminished in primary B-CLL, MEC-1, and NCEB cells after Rituximab incubation, but CD20 mRNA remains stable in NCEB cells in up to 24 days. Incubation with B1 did not show this effect.

Conclusions: Loss or diminished CD20 protein expression after Rituximab therapy is a frequent phenomenon, and time dependent with its maximum within the first three months after therapy. An internalization or intracytoplasmocytic protein degradation of CD20 has not been observed in cell lines. The CD20 "downregulation" is most probably due to B-cell receptor movements into lipid rafts in the cell membrane since incubation with $\mathrm{B} 1$ antibodies preserves $\mathrm{CD} 20$ detection.

\section{BMS 9}

\section{STROMAL-HEMOPOIETIC INTERACTION IN APLASTIC ANEMIA}

Samia H Rizk*, MD, MPH, Manal W El-Masry, MD, Iman M Mansour, MD, Doha Mokhtar MD, and Ilham Yousry MD

Departments of Clinical Pathology and Pediatrics, Cairo University, Egypt

Background: Aplastic anemia is associated with quantitative and functional abnormalities in the hematopoietic stem cell compartment. Activated type 1 cytotoxic $\mathrm{T}$ cells and type 1 cytokines have been implicated. $\gamma$-Interferon (and tumor necrosis factor- $\alpha$ ) reduces the numbers of human hematopoietic progenitor-derived colonies in vitro and efficiently induce apoptosis in CD34 target cells. Also, sustained exposure to low concentrations of $\gamma$-interferon markedly reduces the output of long-term culture-initiating cells (LTC-IC), consistent with local amplification of toxicity in the marrow milieu.

Methodology: In order to estimate the effect of intrinsic inhibitory cytokines on the primitive BM stem cells of aplastic anemia, we studied 14 cases with newly diagnosed acquired aplastic anemia as well as eight controls with non aplastic non infiltrated marrows. Longterm bone marrow culture (LTBMC) was performed for both cases and controls and the loss of CFUs was assessed weekly for five weeks. The degree of apoptosis was also studied in the remaining elements at the end of $4^{\text {th week }}$ using TUNEL staining method.

Results: We demonstrated a significant reduction of the number $(\mathrm{p}=$ $0.015)$ and maintenance $(p=0.005)$ of CFUs in LTBMC of aplastic anemia patients' bone marrows, in comparison to normal controls. The weekly loss of CFUs was also significant till the end of the $5^{\text {th week }}(\mathrm{P}>0.0001)$. The percentage of apoptotic cells in AA patients was higher than that of controls with a significant difference regarding the number of apoptotic colony / 10 colonies $(\mathrm{P}=0.027)$, but not regarding the number of apoptotic cells/ colony.

Conclusion: Immune reaction mediated by cytotoxic T-cells is suggested to be a leading cause for marrow hypoplasia. 


\section{BMS 10}

\section{CCL3 AND CCL4 FACILITATE MICROENVIRONMENTAL INTERACTIONS OF CLL CELLS VIA THE CD49d/VCAM PAIR.}

*Claudio Tripodo ${ }^{1}$, Antonella Zucchetto ${ }^{2}$, Fleur Bossi ${ }^{3}$, Dania Benedetti ${ }^{2}$, Riccardo Bomben ${ }^{2}$, Michele Dal Bo ${ }^{2}$, Daniela Marconi ${ }^{4}$, Massimo Degan ${ }^{2}$, Giovanni Del Poeta ${ }^{5}$, Silvia Deaglio ${ }^{6}$, Gianluca Gaidano $^{7}$, Francesco Tedesco ${ }^{3}$, Fabio Malavasi ${ }^{6}$, Vito Franco ${ }^{1}$, Valter Gattei $^{2}$

${ }^{1}$ Dipartimento di Patologia Umana, Università degli Studi di Palermo, Palermo; ${ }^{2}$ Servizio di Onco-Ematologia Clinico-Sperimentale, Centro di Riferimento Oncologico, IRCCS, Aviano; ${ }^{3}$ Laboratorio di Immunologia, Dipartimento di Fisiologia e Patologia, Università degli Studi di Trieste, Trieste; ${ }^{4}$ Istituto Nazionale di Fisica Nucleare (INFN), Sez. Bologna, Università degli Studi di Bologna; ${ }^{5}$ Ospedale S. Eugenio, Università di Tor Vergata, Roma; ${ }^{6}$ Laboratorio di Immunogenetica, Università degli Studi di Torino; ${ }^{7}$ Divisione di Ematologia, Dipartimento di Medicina Clinica e Sperimentale \& IRCAD, Università degli Studi del Piemonte Orientale Amedeo Avogadro, Novara, Italy.

Background: CD49d and CD38 have a negative prognostic impact on CLL. These two markers share an adhesive function and could mediate interactions between CLL cells and bone marrow (BM) microenvironment, providing proliferation and survival signals for the neoplastic component.

Purpose: We aimed to find a link between CD38 and CD49d that may explain their negative impact in CLL, focusing on genes involved in mechanism(s) regulating CLL-cells/BM-stroma interactions.

Methods: i) Gene-expression profiling performed using mRNA derived from purified CLL cells of $11 \mathrm{CD} 38+\mathrm{CD} 49 \mathrm{~d}+$ and 15 CD38-CD49d- cases; ii) Functional and validation experiments performed by flow-cytometry, real-time quantitative PCR (RTQPCR), ELISA, and immunohistochemistry (IHC).

Results: CCL3 and CCL4 chemokine genes were found up-regulated in $\mathrm{CD} 38+\mathrm{CD} 49 \mathrm{~d}+$ cases, this over-expression being confirmed by RTQ-PCR. In vitro experiments on CD38+CD49d+ CLL-cells cultured for $14(\mathrm{t} 14)$ and 24 hours (t24) in the presence of agonist (IB4) anti-CD38 monoclonal antibodies, demonstrated up-regulation of CCL3/CCL4 mRNA at t14 (by RTQ-PCR, $\mathrm{p}=0.005$ ), and increased release of CCL3/CCL4 protein at 24 (by ELISA, $p=0.01$ ). Consistently, CCL3 was detected by immuno-histochemistry (IHC) in neoplastic cells from BM biopsies (BMBs) of CD38+CD49d+ but not CD38-CD49dCLL. High expression of CCL3/CCL4 receptors CCR1 and CCR5 was found on CLL-derived monocytes and in-vitro differentiated macrophages. Accordingly, a higher number of infiltrating CD68+ macrophages was found in $\mathrm{CD} 38+\mathrm{CD} 49 \mathrm{~d}+$ compared to $\mathrm{CD} 38$ CD49d- cases $(p=0.013)$, suggesting a role of these cells as target for CLL-derived chemokines. Parallel experiments showed that conditioned media from CCL3-stimulated macrophages induced expression of the CD49d-ligand VCAM-1 in human endothelial cells. Consistently, a denser meshwork of VCAM-1+ cells was detected by IHC in CD38+ $\mathrm{CD} 49 \mathrm{~d}+$ as compared to CD38-CD49d- infiltrates ( $\mathrm{p}=0.002$ ).

Conclusion: These findings identify a novel functional cross-talk between CD38+CD49d+CLL cells and cells of the tumor microenvironment, this interplay eventually affecting survival and recirculation of tumor cells via the CD49d/VCAM pair.
BMS 11

\section{LYMPHOPLASMACYTIC LYMPHOMA RESPONDING TO IMATINIB: A POSSIBLE ROLE FOR MAST CELLS IN LYMPHOPLASMACYTIC LYMPHOMA}

\author{
L Tsao, J Chapman, J Horwitz, *CV Cotta
}

Background: Experiments have shown that mast cells directly facilitate the proliferation of lymphoplasmacytic lymphoma cells. Other studies have shown that follicular lymphomas with low density of mast cells have a better prognosis than those with high density. The possible mechanism is the low density of T-regs in these tumors, as mast cells are, at least partially, responsible for the proliferation of $\mathrm{T}$ regs. Since imatinib inhibits mast cell proliferation, there may be a role for imatinib in the treatment of low grade lymphomas.

Purpose: Demonstrate the effect of imatinib on lymphoplasmacytic lymphoma, as monitored by total plasma protein level.

Description of results: A 62 year old man was enrolled on a clinical trial investigating imatinib in idiopathic fibrosing alveolitis. At the onset of the study, he had increased total serum proteins $(9.2 \mathrm{~g} / \mathrm{dL})$. Immediately after treatment, the total serum proteins decreased $(8.1 \mathrm{~g} /$ $\mathrm{dL}$ ), and remained at normal levels $(7.5-8.1 \mathrm{~g} / \mathrm{dL})$ for the duration of the treatment (two years). After imatinib was discontinued, the total serum protein level became elevated $(9.3 \mathrm{~g} / \mathrm{dL})$ with an increase in serum viscosity. The cause of this elevation was a monoclonal IgM (IgM kappa, $3.78 \mathrm{~g} / \mathrm{dL}$ ). The bone marrow biopsy showed lymphoplasmacytic lymphoma.

Conclusion: While the etiology of the increased total serum proteins was not initially established, it was most likely due to the underlying lymphoplasmacytic lymphoma present before and during the treatment with imatinib. The correlation between the treatment schedule and total serum protein level suggests that imatinib could play a role in the treatment of lymphoplasmacytic lymphoma. The mechanisms through which imatinib controls lymphoplasmacytic lymphoma could include inhibition of mast cell proliferation and direct inhibition of pathways controlling lymphoplasmacytic lymphoma cell proliferation.

\section{BMS 12}

\section{ANGIOGENESIS IN CHRONIC MYELOPROLIFERATIVE DISEASES IS INDEPENDENT OF THE $J A K 2$ STATUS}

\author{
*A. Tzankov, S. Dirnhofer, A. Gratwohl, R. Skoda, A. Tichelli, M. \\ Medinger
}

Dept. of Pathology and Hematology, University Hospital Basel, Switzerland

Background: Chronic myeloproliferative diseases (CMPD) are hematopoietic stem cell neoplasias characterized by bone marrow outgrowth with maturation of one ore more myeloid lineages, insidious onset with the potential for progression and transformation and accompanying myelofibrosis. In over $50 \%$ of $\mathrm{Ph}^{-} \mathrm{CMPD}$ cases the hematopoietic precursors harbor an acquired gain-of-function mutation in the JAK2 gene resulting in a selective growth advantage as compared to precursor cells with the wild-type gene. In addition, 
fibrogenic and angiogenic cytokines are aberrantly expressed by clonal megakaryocytes and histiocytes in a significant proportion of cases.

Purpose: We aimed to immunohistochemically study the microvessel density (MVD) and vascular endothelial growth factor (VEGF)expression in $96 \mathrm{Ph}^{-} \mathrm{CMPD}$, including 24 essential thrombocythaemia- (ET), 46 polycythemia vera- (PV), 26 chronic idiopathic myelofibrosis- (CIMF) and 20 control reactive bone marrow cases and to correlate these findings with known biological and clinical key data, particularly with the $J A K 2$ status.

Results: $67 \%$ of the cases (64/96) displayed the V617F mutation in the JAK2 gene. The mean MVD was highest in CIMF (55 microvessels $/ \mathrm{mm} 2)$, compared to PV $(33 / \mathrm{mm} 2)$, ET $(22 / \mathrm{mm} 2)$ and controls $(15 / \mathrm{mm} 2, \mathrm{p}<0.0001)$ as was the expression of VEGF in megakaryocytes and histiocytes ( 15 cells $/ \mathrm{mm} 2$ in CIMF, $13 / \mathrm{mm} 2$ in PV, 12/ $\mathrm{mm} 2$ in ET and $5 / \mathrm{mm} 2$ in controls, $\mathrm{p}=0.003$ ). Increased MVD correlated with the fibrosis degree only in ET and exceeded that of the controls solely in ET cases with fibrosis $\geq$ grade 1 . There were no correlations between MVD or expression of VEGF and JAK2 status, thrombotic or bleeding complications or transformation.

Conclusion: Except for early ET, angiogenesis is increased in $\mathrm{Ph}^{-}$ CMPD, particularly in CIMF. It is independent of the JAK2 status and fibrosis degree (except for early ET). Thus, angiogenesis might potentially serve as $J A K 2$ independent treatment target in $\mathrm{Ph}^{-} \mathrm{CMPD}$, especially in cases with present or imminent fibrosis.

\section{BMS 13}

\section{ROLE OF CD146+ BONE MARROW OSTEOPROGENITORS IN PRIMARY MYELOFIBROSIS.}

Claudio Tripodo ${ }^{1}$, ${ }^{*}$ Andrea Di Bernardo ${ }^{1}$, Maria Paola Ternullo ${ }^{1}$, Carla Guarnotta ${ }^{1}$, Umberto Gianelli ${ }^{2}$, Emanuela Boveri ${ }^{3}$, Rossana Porcasi $^{1}$, Sabrina Ingrao ${ }^{1}$, Ada Maria Florena ${ }^{1}$ and Vito Franco ${ }^{1}$

${ }^{1}$ Department of Human Pathology, Università degli Studi of Palermo, Policlinico Paolo Giaccone, Palermo,Italy; ${ }^{2}$ Pathology Unit, Department of Medicine, Surgery and Odontology, San Paolo Hospital, and Policlinico IRCCS Hospital, Mangiagalli and Regina Elena Foundation, University of Milan,Italy; ${ }^{3}$ Departments of Surgical Pathology and Hematology, University of Pavia Medical School, Fondazione IRCCS Policlinico San Matteo, Pavia, Italy

Background: Primary Myelofibrosis is characterized by a poor prognosis and by the lack of a valid therapeutic approach mainly beacause of the progressive development of reticulin fibrosis leading to bone marrow (BM) failure. BM stromal reaction seems to play a role in the progression of the disease as suggested by both the increase of neoangiogenesis in the different phases and the formation of new bone (osteomyelosclerosis) in the advanced stages. CD146+ bone marrow (BM) stromal cells have been recently recognized as clonogenic osteoprogenitors able to organize a complete hematopoietic microenvironment and induce bone formation.

Purpose of the study: In this study we investigated the contribution of CD146+ BM osteoprogenitors to the stromal remodelling occurring in the different stages of primary myelofibrosis (PMF), by immunohistochemical analysis on BM samples from 32 PMF patients.

Results: We found that CD146+ cells sited at the abluminal side of the $\mathrm{BM}$ vessels and branching among hematopoietic cells, signifi- cantly increased in the advanced stages of PMF ( $p<0.001$ ), forming a dense subendothelial meshwork. Such an increase was associated with both the extent of fibrosis $(r h o=0.916, p<0.0001)$ and the microvascular density (rho=0.883, $\mathrm{p}<0.0001)$. Coherently with a mural cell function, such cells were characterized also by the expression of smooth-muscle actin.

Conclusion: Our data suggest that $\mathrm{CD} 146+$ subendothelial reticular cells may play a role in the stromal remodelling occurring in the progression of PMF through the generation of osteoprogenitor cells responsible for bone formation and by the direct production of reticulin fibers. They could also support BM neoangiogenesis through the Angiopoietin-1/Tie-2 pathway, recently described in $\mathrm{Ph}-$ Chronic Myeloproliferative Disorders therefore representing a rationale for new therapies targeting BM stroma in PMF.

\section{BMS 14}

\section{BONE MARROW HISTOLOGY IN RELATION TO JAK2 V617F MUTATION IN POLYCYTHEMIA VERA AND ESSENTIAL THROMBOCYTHEMIA}

*Parasi-Ismailou $\mathrm{A}^{1}$, Kalagiakou $\mathrm{E}^{2}$, Pantoula $\mathrm{P}^{1}$, Belessi $\mathrm{C}^{2}$, Manioudaki $\mathrm{E}^{2}$, Karayannis $\mathrm{M}^{1}$, Xyristaki $\mathrm{S}^{1}$, Laoutaris $\mathrm{N}^{2}$.

Pathology ${ }^{1}$, Hematology ${ }^{2}$ Department General Hospital of Nikea-Piraeus "Agios Panteleimon", Athens, Greece

Background: Polycythemia vera (PV) and Essential Thrombocythemia (ET) are members of the $\mathrm{Ph}$ - chronic myeloproliferative diseases (CMPDs) characterized by hyperplasia of one or more hematopoietic cell lines, dysplastic features of the megakaryocytic lineage, variable marrow fibrosis and clinical heterogeneity. The identification of JAK2V617F mutation in a high proportion of the Ph- CMPDs has provided new insights into the molecular basis of Ph- CMPDs.

Purpose: Detection of JAK2 mutation in PV and ET patients and possible correlation with BM cellularity, changes in the megakaryocytic lineage and degree of BM fibrosis.

Material: Cellularity and BM Fibrosis (after Gomori's stain) were evaluated and scored, according to the recent European Consensus proposal, in BM trephine biopsies of 30 patients (15 PV and $15 \mathrm{ET}$ ). Megakaryocytic changes (clustering, morphology) were interpreted after LAT-1 protein immunostain. The histological parameters were evaluated by two pathologists in a blinded fashion. JAK2-V617F mutation was detected, using allele specific PCR.

Results: The V617F mutation was detected in 20 cases, 13/15(86.7\%) PV and 7/15(46.7\%) ET patients. A positive correlation was found between JAK2 positivity and BM cellularity. All 12 cases with marked increase in cellularity carried the JAK2 mutation, while none of the 5 cases with mild or no increase in cellularity carried the mutation $(p=$ 0.001 ). Moderate to marked increase in cellularity was observed in all JAK2+ PV and ET cases. No correlation of the JAK2 mutation with the degree of fibrosis and the megakaryocytic changes was observed. Conclusion: Our results indicate that JAK2-V617F mutation could correlate with the clinical and laboratory heterogeneity of ET. 


\section{BMS 15}

\section{PRIMARY BONE MARROW LYMPHOMAS (PBML): CLINICAL PRESENTATION, HISTOPATHOLOGICAL FEATURES AND OUTCOME (IELSG \#29)}

*M. Rozman ${ }^{1}$, A. Martínez ${ }^{1}$, C. Agostinelli ${ }^{2}$, K. M. Hebeda ${ }^{3}$, E. Matutes $^{4}$, M. Ponzoni ${ }^{5 \mathrm{a}, 5 \mathrm{~b}}$, A. Ferreri ${ }^{5 \mathrm{a}, 5 \mathrm{c}}$, C. Campidelli ${ }^{2}$, B. Espinet $^{6}$, G. Perea $^{7}$, A. Acevedo ${ }^{8}$, S. Pileri ${ }^{2}$, A. López-Guillermo ${ }^{1}$, E. Campo ${ }^{1}$, on behalf of the International Extranodal Lymphoma Study Group.

${ }^{1}$ Hematopathology Unit and Hematology Department, Hospital Clínic, DIBAPS, University of Barcelona, Spain; ${ }^{2}$ Haematopathology and Hematology Depatment, Bologna University School of Medicine, Bologna, Italy; ${ }^{3}$ Department of Pathology, Radboud University Nijmegen Medical Centre, Nijmegen, The Netherlands; ${ }^{4}$ HaematoOncology Unit. Royal Marsden Hospital/Institute of Cancer Research, London, UK; ${ }^{5 a}$ Unit of Lymphoid malignancies, ${ }^{5 b}$ Pathology Unit and ${ }^{5 c}$ Medical Oncology Units, San Raffaele Scientific Institute, Milan, Italy; ${ }^{6}$ Cytogenetics and Molecular Biology Laboratory, Pathology Service, Hospital del Mar, Barcelona, Spain; ${ }^{7}$ Laboratory Department, Corporació Sanitaria Parc Taulí, Sabadell (Barcelona), Spain; ${ }^{8} \mathrm{~Pa}-$ thology Department, Hospital Quirón Madrid, Madrid, Spain

Background: PBML are rare and only few cases are reported in the literature.

Purpose of the study: To determine whether PBML may have specific clinico-pathological features and distinct outcome, we have retrospectively reviewed cases of PBML among eight European institutions. Including criteria were: 1) isolated BM involvement, 2) no evidence of extra-marrow dissemination on imaging studies, 3) no evidence of splenic, liver or other organ infiltration, 4) absence of localized bone tumours, and 5) exclusion of lymphomas primarily involving BM (SLL/CLL, LPL, MCL, SMZL, Burkitt and ALL).

Results: Seventeen cases fulfilled the inclusion criteria, and complete clinical data were available for 16 of them. The median age was 64 (29-91) years without sex predominance; B-symptoms were present in $69 \%$, leukopenia in $31 \%$, anemia in $46 \%$ and thrombocytopenia in $50 \%$. Three cases $(19 \%)$ had blood involvement. High serum LDH was observed in $79 \%$ cases. Twelve patients received CHOP-like or R-CHOP-like regimens; the complete response rate was $36 \%$. Median overall survival (OS) was 1.8 years. Platelet count $<100 \times 10^{9} / \mathrm{L}$ and high serum LDH predicted poor OS.

Histologically, 12/17 cases had large B-cell lymphoma (6 nodular / 6 diffuse infiltration pattern). Among these, a germinal centre (GC) origin was demonstrated in 2 and excluded in 1, whereas 2 cases were T-cell rich B-NHL. The GC-associated features could not be assessed in 9 cases. The remaining 5 cases had histology consistent with FL (3) and PTCL (2). The 3 FL had paratrabecular infiltration, rearrangement of bcl-2 and 2 had peripheral blood involvement. The 2 PTCL showed an interstitial/diffuse infiltration pattern, bone marrow fibrosis, mild eosinophilia, and PD-1 expression consistent with a derivation from GC-T-cells.

Conclusion: PBML is a very uncommon extra-nodal lymphoma often associated with cytopenias, aggressive disease, poor outcome and heterogeneous histological features, some of them revealing a putative germinal centre origin.
BMS 16

\section{BONE MARROW PATHOLOGY CONTRIBUTES TO "IN SITU" UNDERSTANDING OF CHRONIC LYMPHOCYTIC LEUKEMIA IMMUNOPATHOGENESIS: ANALYSIS OF A SERIES OF 112 PATIENTS}

*Maria Fameli ${ }^{1}$, Niki Stavroyianni ${ }^{2}$, Ioanna Athanasiadou ${ }^{2}$, Evi Pouliou ${ }^{1}$, Kostas Stamatopoulos ${ }^{2}$, Achilles Anagnostopoulos ${ }^{2}$, Athanasios Fassas ${ }^{2}$, Theodora Papadaki ${ }^{1}$

${ }^{1}$ Department of Hematopathology, Evangelismos Hospital, Athens; ${ }^{2}$ Department of Hematology and HCT Unit G. Papanikolaou Hospital, Thessaloniki; all Greece

We analyzed bone marrow biopsy (BMB) samples from 112 patients with CLL and explored associations with clinical features and outcome, CD38 expression, and IGHV mutation status. Patient group: $\mathrm{M}: \mathrm{F}=66: 46$, median age: 66 years, Binet stage-A/B/C: 88/19/5. Peripheral blood immunophenotype: (1) CD38+: 42/112 cases (2) IgG+: 16/103 cases. Mutated/unmutated IGHV genes (M-IGHV/AIGHV): 73/39 cases. Formalin-fixed, decalcified, paraffin-embedded sections of BMBs were examined: (1) morphologically (H\&E); (2) immunohistochemically ( $\mathrm{ABC}$ technique). The following antibodies were used: CD20, CD79a, CD3, BCL-2, CD5, CD23, SIg/CIg (k/1/m/ d/g/a), CD27, myeloperoxidase, PGM1 (CD68), glycophorin-C, CD61. All cases showed neoplastic small lymphocytic infiltration $(20-95 \%$ of BM cellularity) by CD20+CD79a+CD5+CD23+cells. Medium- or large-sized cells were admixed in 6 and 2 cases, respectively. A moderate number of monocytoid-like cells with clear cytoplasm were identified in 4 cases. Finally, 6 cases exhibited lymphoplasmocytoid differentiation in a small proportion of the neoplastic population. Three patterns of neoplastic lymphocytic infiltration were identified: (1) interstitial: 50 cases; (2) nodular/ nodular+interstitial: 35 cases; (3) diffuse: 27 cases. Moderate-tosignificant reduction of the granulocytic series was observed in 54/112 cases. The remaining cases showed hyperplastic granulocytic series with a left shift and, occasionally, dysplastic changes of mature forms. Hyperplasia of the erythroid and megakaryocytic series -often with dysplastic changes- with normal/decreased hemoglobin levels and platelet counts was observed in 64/112 and 85/112 cases, respectively; the remaining cases showed moderate-to-significant reduction of either series. Significant correlations were identified between: (i) nodular infiltration and M-IGHV genes $(p<0.001)$; (ii) diffuse infiltration and shorter time-to-progression $(\mathrm{p}=0.05)$.

In conclusion, $\mathrm{BMB}$ examination: (1) permits identification of the rare CLL subtype with plasmocytoid differentiation (WHO); (2) allows evaluation the BM stroma and the hematopoietic marrow and provides important evidence for CLL-associated hematopoietic autoimmunity. Finally, the favorable prognosis of the nodular pattern of infiltration in CLL may be interpreted in view of its association with M-IGHV genes. 


\section{BMS 17}

\section{MARGINAL ZONE LYMPHOMAS (MZL) PRESENTING WITH ABSOLUTE MONOCLONAL LYMPHOCYTOSIS AND/OR MONOCLONAL GAMMOPATHY. A REPORT OF 8 CASES}

George Kanellis ${ }^{1}$, Ekaterini Stephanoudaki ${ }^{2}$, Gariphalia Kokkini ${ }^{3}$, Eleni Papadaki ${ }^{4},{ }^{*}$ Theodora Papadaki ${ }^{5}$

${ }^{1}$ Cytology Department, "Evangelismos" Hospital, Athens; ${ }^{2}$ Department of Hematology, "Amalia Fleming" Hospital, Athens; ${ }^{3}$ Department of Hematology, "Sismanoglio" Hospital, Athens; ${ }^{4}$ Department of Hematology University of Crete School of Medicine; ${ }^{5}$ Department of Hematopathology "Evangelismos" Hospital, Athens; all Greece.

Introduction: Lymphocytosis and/or monoclonal gammopathy can be the presenting findings in a number of malignant B-cell lymphoproliferative diseases. Furthermore, a number of different disorders with monoclonal proliferation of plasma cells (PC) are encountered in routine bone marrow $(\mathrm{BM})$ evaluation, some of which primarily arise from the BM. However, exclusively clonal PCs or plasmocytoid lymphocytes admixed with a high number of clonal PCs may be seen in the context of secondary involvement of the BM by an occult Bcell lymphoma, thus hindering a definitive diagnosis.

The purpose of the present study was to evaluate the morphological and immunohistochemical findings of 8 cases of occult MZL (MALT type $\mathrm{x} 6$, Nodal $\mathrm{x} 2$ ) presenting with absolute lymphocytosis and/or monoclonal gammopathy, in which the BM was the first histopathologically assessed tissue.

Methods: Paraffin sections from BM biopsies (x8) as well as nodal and extranodal tissues (stomach $\mathrm{x} 4$, lung $\mathrm{x} 1$, oral mucosa $\mathrm{x} 1$, lymph nodes $\mathrm{x} 2$ ) were studied morphologically (H\&E) and immunohistochemically for CD20, CD79 $\alpha$, CD3, CD5, CD23, Cyclin D1, CD43, MUM-1, CD138, CD55, SIg/CIg $(\kappa, \lambda, \gamma, \mu, \alpha)$, CD21.

Results: Demographics, presenting symptoms and morphological and immunohistochemical findings are summarized in Table 1.
Conclusions: Our observations,

1. support the close association between the cell of origin of MALT or nodal MZL and their plasmacytic component in the concept of the capacity of their normal counterparts, MZ B-cells, to differentiate into antibody secreting plasma cells.

2. confirm recent data concerning the tendency of extranodal and or nodal MZL with plasmacytic differentiation to be associated with disseminated disease at initial presentation.

3. emphasize the value of $\mathrm{BMB}$ in the monitoring of patients presenting with monoclonal lymphocytosis and or gammopathy.

4. suggest that pathologists should be alerted that clonal lymphoplacytic and/or pure plasmacytic BMB infiltrations could be the first presentation of occult extranodal or nodal MZL.

\section{BMS 18}

\section{Transformation of Marginal Zone Lymphomas to pure plasma cell Histology following treatment with the anti-CD20 antibody Rituximab}

Kypros Dimosthenous ${ }^{1}$, Asimina Papanikolaou ${ }^{2}$, Ioanna Athanasiadou ${ }^{3}$, Ekaterini Stephanoudaki ${ }^{4}$, Gariphalia kokkini ${ }^{5}$, Eleni Papadaki ${ }^{6}$, *Theodora Papadaki ${ }^{2}$.

${ }^{1}$ Cytology Department Laiko Hospital, Athens; ${ }^{2}$ Department of Hematopathology Evangelismos Hospital, Athens; ${ }^{3}$ Department of Hematology and HCT unit G. Papanikolaou Hospital, Thessaloniki; ${ }^{4}$ Department of Hematology Amalia Phleming Hospital, Athens; ${ }^{5}$ Department of Hematology Sismanoglion Hospital, Athens; ${ }^{6}$ Department of Hematology University of Crete, School of Medicine; all Greece.

Background: Marginal Zone Lymphomas (MZL) [extranodal MALTtype, nodal, splenic] putatively arise from marginal zone B-cells which are ontogenetically related to antibody secreting plasma cells.

Table 1

\begin{tabular}{|c|c|c|c|c|}
\hline Case & Lymphocytosis & $\begin{array}{l}\text { Monoclonal } \\
\text { paraprotein }\end{array}$ & ВMB & Tissue \\
\hline 1 & + & - & $\begin{array}{l}\text { Clonal } \mathrm{M}(\lambda) \mathrm{B} \text {-cell and } \mathrm{PC} \\
\text { infiltration }\end{array}$ & BALT lymphoma with PC differentiation and $\mathrm{M}(\lambda)$ clonality \\
\hline 2 & + & - & $\begin{array}{l}\text { Clonal } \mathrm{G}(\mathrm{K}) \mathrm{B} \text {-cell and } \mathrm{PC} \\
\text { infiltration }\end{array}$ & Nodal MZL with PC differentiation and $\mathrm{G}(\mathrm{K})$ clonality \\
\hline 3 & - & $\mathrm{G}(\kappa)$ & $\begin{array}{l}\text { Clonal } \mathrm{G}(\mathrm{K}) \mathrm{B} \text {-cell and } \mathrm{PC} \\
\text { infiltration }\end{array}$ & $\begin{array}{l}\text { MALT lymphoma of oral mucosa with PC differentiation and } \\
\text { G(K) clonality }\end{array}$ \\
\hline 4 & - & $\mathrm{M}(\mathrm{k})$ & $\begin{array}{l}\text { Clonal } \mathrm{M}(\mathrm{K}) \mathrm{B} \text {-cell and } \mathrm{PC} \\
\text { infiltration }\end{array}$ & $\begin{array}{l}\text { MALT lymphoma of stomach with PC differentiation and } \\
\text { M() clonality }\end{array}$ \\
\hline 5 & + & $\mathrm{M}(\mathrm{k})$ & $\begin{array}{l}\text { Clonal } \mathrm{M}(\mathrm{K}) \mathrm{B}-\text { cell and } \mathrm{PC} \\
\text { infiltration }\end{array}$ & Nodal MZL with PC differentiation and $\mathrm{M}(\mathrm{K})$ clonality \\
\hline 6 & - & $\mathrm{G}(\kappa)$ & $\begin{array}{l}\text { Clonal } \mathrm{G}(\mathrm{K}) \mathrm{B} \text {-cell and } \mathrm{PC} \\
\text { infiltration }\end{array}$ & $\begin{array}{l}\text { MALT lymphoma of stomach with PC differentiation and } \\
\text { G(K) clonality }\end{array}$ \\
\hline 7 & + & $\mathrm{A}(\mathrm{K})$ & $\begin{array}{l}\text { Clonal } \mathrm{A}(\mathrm{K}) \mathrm{B} \text {-cell and } \mathrm{PC} \\
\text { infiltration }\end{array}$ & $\begin{array}{l}\text { MALT lymphoma of stomach with PC differentiation and } \\
\mathrm{A}(\mathrm{K}) \text { clonality }\end{array}$ \\
\hline 8 & + & - & $\begin{array}{l}\text { Clonal } \mathrm{M}(\mathrm{\kappa}) \mathrm{B}-\text { cell and } \mathrm{PC} \\
\text { infiltration }\end{array}$ & $\begin{array}{l}\text { MALT lymphoma of stomach with PC differentiation and } \\
\mathrm{M}(\mathrm{K}) \text { clonality }\end{array}$ \\
\hline
\end{tabular}


This may also interpret their variable plasmacytic differentiation. In contrast to the almost ubiquitous CD-20 negativity of plasma cells, MZL cells express CD20 and the anti-CD20 antibody Rituximab is reported as an active treatment in patients with MZL.

Purpose of the study: Presentation of 6 MZL patients with plasmatic differentiation who were treated with Rituximab and subsequently developed a purely clonal plasmacytic infiltration of the bone marrow (BM), clonally identical to the neoplastic lymphoplasmacytic infiltration in the initial biopsy sites (nodal/extranodal tissues as well as $\mathrm{BM})$.

Materials and Methods: 4 males and 2 females, aged 58-75 years. Extranodal lymphoma (x3), splenic (x1), nodal (2), BM biopsy (BMB) (x6) samples were available for diagnosis.Immunohistochemical panel: CD20, CD79, CD138, SIg/Cig, CD56, CD3, CD21, CD5, CD23, Cyclin D1, DBA44, MPO, LAT, Glycophorin C.

Results: The results are summarized in table 1

Conclusions: Our observations:

1) Confirm the close association between the cell of origin of MZL and their plasmacytic component, in line with the normal B-cell differentiation.

2) Demonstrate that Rituximab can profoundly alter the histologic features of MZL with plasmacytic differentiation. The most likely explanation for these unusual histologic changes is eradication of the CD20-positive MZL cells with consecutive "overgrowth" of the CD20-negative plasma cellular component of the tumor, as evidenced by the identical clonality of pre- and post- Rituximab analyzed tissues.

3) Suggest that careful re-assessment and biopsy of the "MZL" persisting or relapsing in the BM or other sites outside the BM post-Rituximab, is mandatory, in order to avoid potentially ineffective treatment approaches, such as re-induction with Rituximab.
BMS 19

\section{LYMPHOCYTE SUBPOPULATIONS AND BONE MARROW HISTOPATHOLOGY IN RITUXIMAB-TREATED LYMPHOMA PATIENTS WITH LATE-ONSET NEUTROPENIA (LON)}

*Kostas Stamatopoulos ${ }^{1}$, Ioanna Athanasiadou ${ }^{1}$, Charalambos Pontikoglou $^{2}$, Niki Stavroyianni ${ }^{1}$, Katerina Pyrovolaki ${ }^{3}$, George Paterakis ${ }^{4}$, Achilles Anagnostopoulos ${ }^{1}$, Helen Papadaki ${ }^{3}$, Theodora Papadaki $^{2}$,

${ }^{1}$ Department of Hematology and HCT Unit G. Papanikolaou Hospital,
Thessaloniki; ${ }^{2}$ Department of Hematology, University of Crete School
of Medicine, Heraklion, Crete; ${ }^{3}$ Department of Hematopathology,
Evangelismos Hospital, Athens; ${ }^{4}$ Immunology Laboratory, G.
Gennimatas Hospital, Athens; all Greece

LON is a complication of Rituximab of yet unknown pathophysiology. We investigated potential underlying mechanisms in 12 patients with various non-Hodgkin lymphoma (NHL) subtypes who developed LON without identifiable causes at a median of 95 days after completion of the intended treatment with Rituximab \pm chemotherapy. Similarly treated NHL patients who did not develop LON were used as controls. Ten of 12 patients with LON and 33/38 controls developed profound B-cell depletion. Inverted $\mathrm{CD} 4 / \mathrm{CD} 8$ cell ratios were observed in 10/12 LON cases vs. $13 / 38$ controls $(p<0.01)$. CD8 cells $>1.0 \times 10^{9} / \mathrm{L}$ were identified in $8 / 12 \mathrm{LON}$ cases vs. $8 / 38$ controls $(\mathrm{p}<0.01)$. T-LGL proliferation $(\mathrm{CD} 3+\mathrm{CD} 8+\mathrm{CD} 57+$ cells $>30 \%)$ was observed in $7 / 12$ LON cases vs. 14/38 controls $(p=0.1885)$. BM biopsy samples from 10/12 LON cases were examined at onset of

Table 1

\begin{tabular}{|c|c|c|c|c|c|}
\hline & Age & Sex & Sample & BMB before Rituximab & BMB after Rituximab \\
\hline 1 & 70 & $\mathrm{M}$ & Tonsil: MALT lymphoma with PCD/CIgM (к) & $\begin{array}{l}\text { B-cell lymphoid infiltrate with } \\
\text { PCD/CIgM (k) }\end{array}$ & Pure plasma cell infiltration CIgM (K) \\
\hline 2 & 75 & $\mathrm{M}$ & Stomach: MALT lymphoma with PCD/CIgM (к) & $\begin{array}{l}\text { B-cell lymphoid infiltrate with } \\
\text { PCD/CIgM (k) }\end{array}$ & Pure plasma cell infiltration CIgM (K) \\
\hline 3 & 60 & $\mathrm{M}$ & Stomach: MALT lymphoma with PCD/ CIgA (k) & $\begin{array}{l}\text { B-cell lymphoid infiltrate with } \\
\text { PCD/CIgA (K) }\end{array}$ & Pure plasma cell infiltration CIgA $(\mathrm{K})$ \\
\hline 4 & 64 & $\mathrm{M}$ & Lymph node: NMZL with PCD/CIgM $(\lambda)$ & $\begin{array}{l}\text { B-cell lymphoid infiltrate with } \\
\text { PCD/CIgM }(\lambda)\end{array}$ & Pure plasma cell infiltration $\operatorname{CIgM}(\lambda)$ \\
\hline 5 & 72 & $\mathrm{~F}$ & Lymph node: NMZL with PCD/CIgG (k) & $\begin{array}{l}\text { B-cell lymphoid infiltrate with } \\
\text { PCD/CIgG (K) }\end{array}$ & Pure plasma cell infiltration CIgG $(\kappa)$ \\
\hline 6 & 58 & $\mathrm{~F}$ & Spleen: SMZL with PCD/ CIgM (к) & $\begin{array}{l}\text { B-cell lymphoid infiltrate with } \\
\text { PCD/CIgM (k) }\end{array}$ & Pure plasma cell infiltration CIgM $(K)$ \\
\hline
\end{tabular}


neutropenia. Mild-to-moderate lymphocytic infiltration with predominantly nodular and/or interstitial growth was observed in all cases. Lymphoid aggregates lacked CD20+ or CD79a+ B-cells and were composed entirely of $\mathrm{CD} 3+\mathrm{CD} 45 \mathrm{RO}+\mathrm{CD} 43+\mathrm{T}$-cells. In the control group, BM infiltration by $\mathrm{T}$ cells with similar features as above was observed in 18/27 cases. Seven of 10 LON patients showed moderateto-significant hypoplasia of the granulocytic series; the remaining three exhibited hyperplasia. All ten LON patients had pronounced shift-to-the-left, extending to maturation arrest in 6/10 cases. In contrast, 21/27 control cases (78\%) showed granulocytic hyperplasia; the remaining cases were normal or showed granulocytic hypoplasia. As in LON cases, control cases exhibited different degrees of shift-tothe-left of the granulocytic series. Hyperplasia of the erythroid and megakaryocytic series with dyserythropoiesis and dysmegakaryopoeisis, respectively, was identified in all LON cases and most controls. In all cases with repeat samples, MDS-like changes eventually resolved. We conclude that T-cell mediated autoimmune myelopathy/myelodysplasia associated with MDS-like changes of all myeloid series is critically implicated in the pathophysiology of Rituximab-related LON, at least in a subset of cases.

\section{BMS 20}

\section{COMPARISON OF CD23 EXPRESSION IN CHRONIC LYMPHOCYTIC LEUKEMIA (CLL) AND MANTLE CELL LYMPHOMA (MCL). CLINICAL UTILITY OF A THRESHOLD VALUE FOR EXPRESSION INTENSITY.}

Ravi Patel ${ }^{2}$, Joanne Luider ${ }^{1}$, Xiu Jiang, Iwona Auer ${ }^{1}$, Adnan Mansoor ${ }^{1}$.

Department of Pathology and Laboratory Medicine, University of Calgary \& Calgary Laboratory Services and ${ }^{2}$,Master in Biotechnology program University of Calgary. Calgary, Alberta, Canada.

Objective: CD19/CD5+ B-cell lymphoma represents a heterogeneous group, however, most common differential diagnosis is CLL and MCL. WHO criteria identify the leukemic cells in CLL to be positive for CD19/CD5/CD23. Among MCL patients (pts) CD23 is either absent or weakly expressed. Differential expression of CD23 in CLL is well known and recently; some reports have indicated expression of CD23 in MCL pts. Thus mere positive and negative expression of CD23 fails to differentiate between CLL and MCL by flow-cytometry. The purpose of this study is to determine a threshold value for CD23 expression using flow cytometry, a value which can be adopted in clinical practice to differentiate between CLL and MCL.

Methods: Pts from Calgary Health Region (CHR) with confirmed diagnosis by morphology /immunohistochemistry / FISH were included in the analysis. Cyclin D1 and $\mathrm{t}(11 ; 14) \mathrm{FISH}$ was used to distinguish between MCL and CLL pts. Peripheral blood, bone marrow, lymph node tissue etc were stained with a 5-colour panel consisting of FMC7-FITC, CD43-PE, CD23-ECD, CD5-PC5 and CD19-PC7. Neoplastic cells were identified using a CD5/CD19 gate; median fluorescent intensities (MFI) as well as percentages of positive cells (\% positive) were recorded utilising FC500 flow-cytometer.

Results: A total of 154 pts (131 CLL and $23 \mathrm{MCL}$ ) between the ages of 33-89 yrs (median 69yrs) were included in the analysis. The samples comprised of peripheral blood $(108 ; 70 \%)$, bone marrow (34, $21 \%)$, lymph node $(7,5 \%)$ and others $(5,4 \%)$. Using a MFI threshold of 0.700 to determine positivity, CD23 expression was able to differentiate between CLL and MCL with a sensitivity of $96.1 \%$ and a specificity of $91.7 \%$. The Chi-square test revealed a $p$ value of $<0.0001$ indicating a significant association between CD23 expression and type of disease (CLL or MCL). Similarly, a threshold of $40 \%$ to determine positivity, revealed a sensitivity of $94.7 \%$ and a specificity of $95.7 \%$. The Chi-square $p$ value was $<0.0001$.

Conclusions: Increased CD23 expression with higher intensity is significantly more frequent in CLL when compared to MCL. Using a threshold of $40 \%$ positive, CD23 expression by flow-cytometry has significant predictive value in differentiating CLL from MCL.

\section{BMS 21}

\section{THE STROMAL COMPOSITION OF MAST CELL AGGREGATES IN SYSTEMIC MASTOCYTOSIS}

Attilio Orazi ${ }^{1}$, Nahid Nanaji ${ }^{1}$, Gabriela Gheorghe ${ }^{1}$, Amy Chadburn ${ }^{2}$, April Chiu $^{2}$

${ }^{1}$ Indiana University School of Medicine, Indianapolis, IN and ${ }^{2}$ Weill Medical College of Cornell University, New York, NY, USA.

Introduction: Mastocytosis (MC) is a rare stem cell disorder characterized by pathological accumulation of mast cells in one or more organ systems. Mast cells are known to be the source of fibrogenic cytokines including platelet-derived growth factor, transforming growth factor-beta and basic fibroblast growth factor. Tryptase and histamine present in mast cells are also capable of inducing fibrosis. Although systemic MC is characterized by the presence of fibrosis in areas of bone marrow (BM) involvement, very little is known of the stromal composition involved in this response. Design: We investigated the stromal cellular composition and immunophenotypic expression in areas involved by $\mathrm{MC}$ in ten $\mathrm{BM}$ biopsies and one spleen sample with systemic MC. The extracellular matrix (ECM) was evaluated by immunohistochemistry for collagen IV (CIV22), laminin (4C7), and by thrichromic and reticulin staining. The stromal cells were examined with an antibody against low-affinity nerve growth factor receptor (L-NGFR; ME20-4), a marker of BM reticulum cells, periarteriolar, and pericapillary adventitial cells, and dendritic reticulum cells. Smooth muscle actin (SMA; 1A4) was used to identify myofibroblastic differentiation. Five normal BM biopsies were used as controls. The results were also compared with data previously obtained by us in other types of diseases associated with BM fibrosis (e.g. primary myelofibrosis, metastatic malignancies, HIV and granulomatous infections)

Results: In areas of MC involvement, all cases showed moderate to severe reticulin fibrosis which was associated with the presence of mature collagen as demonstrated by thrichrome stain. The fibrotic areas were largely negative for markers of BM stroma cells including L-NGFR and for ECM proteins (other than mature collagen) such as collagen IV or laminin. SMA expression was identified in only three of the cases. Additionally, areas occupied by fibrotic mast cell lesions were largely devoid of neovascularization (microvessels) as seen by CD34 immunostaining (performed in four cases).

Conclusion: Our immunohistochemical results are consistent with a mature fibroblastic response most likely induced by the mast cells perhaps in combination with other cell types (e.g. eosinophils). The paucicity of cells expressing L-NGFR and to a lesser extent SMA and the presence of mature collagen, as shown by thrichrome staining, is 
comparable to what is seen in other pathologic conditions also characterized by advanced BM fibrosis, such as osteosclerotic primary myelofibrosis, well formed granulomas, and in a variety of non hematologic metastatic malignancies.

\section{BMS 22}

\section{THE DIFFERENTIAL DIAGNOSIS BETWEEN "EARLY PHASE" OF POLYCYTHEMIA VERA AND ESSENTIAL THROMBOCYTHEMIA: A DIAGNOSTIC ALGORITHM.}

Umberto Gianelli*, Alessia Moro, Daniela Graziani, Leonardo Boiocchi, Alessandra Iurlo, Claudia Vener, Elisa Fermo, Paola Bianchi, Silvano Bosari, Giorgio Lambertenghi Deliliers, Alberto Zanella, Guido Coggi.

Pathology Unit, Hematology I - Bone Marrow Transplant Unit, Hematology Unit II, University of Milan, DMCO - San Paolo Hospital and Policlinico IRCCS Hospital, Mangiagalli and Regina Elena Foundation, Milan, Italy.

Background: it has been suggested that Polycythemia Vera (PV) could be preceded by an early "latent" phase of the disease (e-PV), in which, by definition, the increase in the red cell mass or hemoglobin level is lower than requested for the PV diagnosis either by the updated diagnostic criteria of the Polycythemia Vera Study Group (PVSG) or by the WHO classification.

Purpose of the study: to identify the diagnostic criteria useful for a diagnosis of e-PV. We examined the clinical, morphological and molecular features of a cohort of patients presenting at the first observation with thrombocytosis, in the range of ET, and upper-limit or increased hemoglobin levels, but lower than necessary for PV diagnosis (e-PV), developing during the course of the follow-up a well-characterized PV. We compare them with two other groups presenting well-defined WHO criteria for the diagnosis of ET and PV. Results: e-PV cases result more similar to PV than to ET. In fact, e$\mathrm{PV}$ patients are characterized by increase of the red cells parameters, splenomegaly $(p<0.05)$ and hepatomegaly $(p=0.038)$, together with a hypercellular bone marrow, due to increased erythropoiesis and granulopoiesis, associated to megakaryocytic hyperplasia, with pleomorphic aggregates $(p<0.001)$. The frequency of JAK2 ${ }^{\mathrm{V} 617 \mathrm{~F}}$ mutation was similar in e-PV $(100 \%)$ and PV patients $(95 \%)$, whereas significantly lower $(54 \%)$ in ET patients $(\mathrm{p}=0.0007)$.

Conclusion: the results of our study suggest that the polycythemic phase of PV can be preceded by an "early" (pre-polycythemic) phase of the disease, frequently presenting with thrombocytosis and mimicking ET. This condition could precede the development of PV for several years. A diagnostic algorithm based on JAK2 mutation screening and bone marrow examination could distinguish classical ET from this "early" phase of PV.
BMS 23

\section{BONE MARROW ANGIOGENESIS IN DIFFUSE LARGE B CELL NON HODGKIN LYMPHOMA}

Perunicic Jovanovic $\mathrm{M}^{*}$, Mihaljevic B, Jakovic LJ, Terzic T, Cemerikic Martinovic V, Gotic M, Boskovic D.

Institute of Hematology, Clinical Center of Serbia, Belgrade, Serbia

Background: Angiogenesis plays an important role in pathophysiology of hematological malignancies. The few studies published on bone marrow angiogenesis in lymphoma have raised the question of whether or not microvessel density (MVD) is associated with more aggressive disease.

The purpose of this study was to investigate the correlation between bone marrow (BM) angiogenesis estimated as MVD, with tumor staging and outcome of DLBCL patients (pts).

Results: Retrospective analysis was performed on 50 DLBCL pts who were treated with rituximab plus CHOP. Gender distribution was 30 male/20 female, mean age of $50 \pm 18.16$ years (17-80). High IPI was present in $36 \%$ of patients. Bone marrow infiltration was presented in $16(32 \%)$ pts.

After 5 years of follow-up, 28 (56\%) pts are still in CR and $22(44 \%)$ pts died. Univariate survival analysis showed that high IPI had statistically significant negative influence on overall and also on event free survival time (log rank, $\mathrm{p}<0.01)$.

Bone marrow vessels were visualized by immunohistochemical staining for $\mathrm{CD} 34$ on slides of formalin-fixed, paraffin-embedded BM biopsies. MVD was calculated by the number of vessels per $400 \times$ high-power field (HPF) in the area of the densest vascularization. Microvessel density in the bone marrow was higher in DLBCL pts with high IPI $(\geq 3)$ compared with pts with low IPI $(<3)$. The results showed a statistically significant difference of MVD in the bone marrow specimens between the pts with vs. pts without tumor infiltration (mean 20.35 vs. $41.78 \mathrm{MVD} / \mathrm{HPF}$ ).

Conclusion: Increased angiogenesis in DLBCL, combined with high IPI, represents possible predictive factors of poor prognosis. Our findings have potential implications for vascular targeting and therapy.

\section{BMS 24}

\section{SYSTEMIC MASTOCYTOSIS ASSOCIATED WITH A CLONAL PLASMA CELL DISORDER}

\author{
K. Sapru ${ }^{1}$, K. Purdy $^{2}$, A. Kew ${ }^{2},{ }^{*}$ R. Juskevicius ${ }^{1}$, R. Liwski $^{1}$
}

Departments of Pathology ${ }^{1}$ and Medicine ${ }^{2}$, QEII Health Sciences Centre, Halifax, Nova Scotia, Canada

Systemic mastocytosis (SM) is a rare neoplastic mast cell disease that can sometimes be associated with clonal hematologic non-mast cell lineage disorders (SM-AHNMD; WHO, 2001). Most commonly, the clonal disorders associated with SM are myeloid neoplasms such as acute myeloid leukemia, myelodysplastic syndrome and chronic myelogenous leukemia. There are very few case reports of an association with lymphoproliferative disorders. Association with clonal plasma cell disorders is even more unusual with only four previously reported cases. Here, we report the case of a 64 year old 
male patient who presented with a 2 year history of rash on the thighs and trunk and no other systemic symptoms. Histologic examination of a skin biopsy showed the rash to be consistent with urticaria pigmentosa. The patient was referred for hematologic assessment to rule out possible bone marrow mastocytosis. Morphologic evaluation of a bone marrow biopsy sample revealed extensive involvement by aggregates of neoplastic mast cells, consistent with systemic mastocytosis. In addition, there was the very unexpected but interesting finding of a co-existent lambda restricted plasmacytosis (30\% marrow involvement). Further evaluation revealed a monoclonal IgG/lambda paraprotein in the serum and possible lytic bone lesions of the calvarium. The findings of both SM and plasma cell myeloma are diagnostic of SM-AHNMD.

In conclusion, in addition to myeloid \& lymphoid neoplasms, systemic mastocytosis can be associated with plasma cell disorders. The pathobiology underlying this association is unclear and needs further study.

\section{$\underline{\text { BMS 25 }}$}

\section{BONE MARROW MICROENVIRONMENT- SUSTAINED LYMPHOMA PERSISTENCE IN FOLLICULAR LYMPHOMA WITH MULTIDIRECTIONAL MULTIDIRECTIONAL CELL MIGRATION BETWEEN LYMPH NODE AND BONE MARROW}

Martin Wartenberg ${ }^{1}$, Christian Meyer zum Bueschenfelde ${ }^{2}$, German $\mathrm{Ott}^{3}$, Andreas Rosenwald ${ }^{4}$, Falko Fend ${ }^{5}$, and Marcus Kremer ${ }^{1}$

Institutes of Pathology, ${ }^{1}$ Technical University of Munich, ${ }^{3}$ RobertBosch Krankenhaus, Stuttgart, ${ }^{4}$ University of Wuerzburg, ${ }^{5}$ University of Tuebingen, and ${ }^{2}$ III Internal Dept., Klinikum rechts der Isar, Munich, Germany

Background: In follicular lymphoma (FL), the initial $t(14 ; 18)$ translocation is believed to occur in the bone marrow (BM), whereas evolution to definite lymphoma is assumed to proceed within the germinal centers of involved lymph nodes (LN). However, details of tumor cell dissemination to or from the BM remain largely unknown. Goals: We performed simultaneous mutational analysis of the $\mathrm{IgH}$ heavy chain gene rearrangement of $\mathrm{LN}$ and BM clones. By generating genealogical trees, we delineated the dissemination and migration of FL cells between LN and BM, and revealed the clonal evolution of BM involvement. Furtheron, we analyzed ongoing somatic hypermutation for the presumed influence of $\mathrm{BM}$ microenvironment on clonal tumor cell evolution.

Design: Sequential biopsies of three FL with simultaneous infiltration of LN and BM were studied, by amplification of the $\operatorname{IgH}$ gene with family specific primers (VHL1-6) against FR1 (in cases with freshfrozen LN) and primers against FR2 (in cases with formalin-fixed, paraffin embedded, and decalcified trephines). Microdissection was used when necessary in BM trephines. Amplification products were cloned, transfected and sequenced. Hierachical genealogical trees were generated by comparative analysis with the germline sequence (NCBI Blast) and by interclonal comparison of the clonal microheterogeneity. Ongoing mutations were analyzed using the modified multinomial Chang and Casali formula.
Results: The obtained IgH rearrangements were identical in all LN and $\mathrm{BM}$ clones of each case, showing the common clonal origin of tumor clones. The sequences within each case differed from each other by point mutations (ranging from 3-100), generating intraclonal sequence diversity. The cases showed tumor cell clusters in the BM derived from the most ancient common LN progenitor cell. The LN and $\mathrm{BM}$ clones shared different numbers of mutations (ranging from $30-60 \%$ ), with early migration from the $\mathrm{LN}$ to the $\mathrm{BM}$, and vice versa. About $80 \%$ of the $\mathrm{IgH}$ sequences from the $\mathrm{LN}$ and about $25 \%$ of the BM showed ongoing somatic hypermutation with evidence for antigen selection.

Conclusion: Our results suggest, that BM involvement in FL is characterized by infiltration of descendants of LN clones. Vivid exchange of different derivates of tumor cell subclones among the two compartments were observed. All BM infiltrates showed ongoing somatic hypermutation, indicating that the BM provides a mutagenic microenvironment, similar to the germinal centers in LN.

BMS 26

\section{A COMPARATIVE STUDY OF BONE MARROW BIOPSY INFILTRATION PATTERNS IN ANGIOIMMUNOBLASTIC T CELL LYMPHOMA AND CLASSICAL HODGKIN LYMPHOMA}

\section{REKHA NAIR*, SINDHU NAIR, NILEENA NAYAK , ELIZABETH} ABRAHAM

Background: This study comes from Regional Cancer Centre (RCC) Trivandrum, South India. Around 300 cases of Non Hodgkin Lymphomas and 100 cases of Hodgkin Lymphomas are diagnosed and treated here every year. Peripheral T cell Lymphomas accounts for $22 \%$ of mature lymphomas here. Anaplastic large cell Lymphoma is the commonest type of PTL (49\%) and Angiommunoblastic T cell Lymphoma is the second commonest (26\%).

Purpose of Study: This study aims at comparing the infiltration patterns of Hodgkin Lymphoma and AILT in trephine biopsy specimens from 2006-2008. We have found more similarities than differences in the infiltrative components of both diseases and hence were taken up for study. Morphology, immunotyping and reticulin staining were done on trephine biopsy specimens.

Summarised Description of Results: 20 cases each of AILT- PTL and Hodgkin Lymphoma (already diagnosed on lymph node biopsy) with marrow biopsy positivity were taken up for study. H \& E stained sections in both diseases showed varying degrees of focal and diffuse patterns of infiltration by a polymorphous population of lymphoid cells, eosinophils, histocytes and occasional mononuclear cells with prominent nucleoli in a fibrotic background. A few of Hodgkin lymphoma cases showed RS like cells.

Immunotyping in both diseases revealed predominant population of CD3 positive $\mathrm{T}$ cells (reactive vs neoplastic), CD30 positive mononuclear cells and very few CD20 positive B lymphocytes.. Reticulin staining showed grade 3 to 4 fibrosis in all cases.

Conclusion: Most cases of AILT and Hodgkin Lymphoma infiltrates in bone marrow biopsies cannot be distinguished from each other except in those cases with identifiable RS cells. We are concluding that no attempt should be made to diagnose and differentiate Hodgkin disease and AILT on trephine biopsy sections. 


\section{BMS 27}

\section{QUANTITATIVE DETECTION OF JAK2 V617F MUTATION IN CHRONIC MYELOPROLIFERATIVE DISORDERS}

*Riera Ludovica, Sismondi Francesca, Tondat Fabrizio, Godio Laura, Pich Achille, Francia di Celle Paola

Department of Biomedical Science and Human Oncology, Center of Experimental Medicine and Research (CeRMS) - University of Torino; San Giovanni Battista Hospital - Torino, Italy.

Background: The 2008 WHO document for the classification of haematological tumors includes revised diagnostic criteria for Chronic Myeloproliferative Disorders (CMPDs). Particularly it underlines the occurrence of JAK2 V617F point mutations, in a high percentage of patients with polycythemia vera (PV), essential thrombocythemia (ET) or primary myelofibrosis (PMF).

Because the mutation may be present only in a small proportion of cells and a correlation between hematological improvement and the reduction in the proportion of the mutated allele has been suggested, quantitative detection methods for JAK2 V617F are required.

Purpose: In this study, we have developed a real-time quantitative allele-specific PCR assay to detect the JAK2 V617F mutation. We have validated our approach by comparing the results with those achieved using two previously standardized methods.

Results: JAK2 mutation testing was performed on total peripheral blood or bone marrow samples using direct sequencing $(n=1321)$ and allele-specific PCR $(n=1224)$ of exon 14 JAK2 gene. These analysis revealed that $478(36.2 \%)$ and $410(33.5 \%)$ cases was mutated respectively.

Moreover, we performed a real-time PCR approach for the quantitative determination of JAK2 V617F mutated allele and detected 113 (29.5\%) mutated cases. A comparison between the three methods lead to $100 \%$ of correlation.

Inside the group of revised diagnosis of CMPD we observed the mutation in $88 \%$ of PV, $64 \%$ of ET and $72.5 \%$ of PMF. Mutational load was not significantly increased among cases with PV compared with ET, while a small number of patients with PMF showed increases mutation loads compared with ET $(\mathrm{P}=0.002)$.

Conclusion: We have developed and validated a quantitative real-time PCR approach to the detection of the V617F mutation of the JAK2 gene associated with CMPDs. The development of an approach that is able to quantities JAK2 mutation load may improve the diagnostic definition and may have clinical relevance in the future.

\section{BMS 28}

\section{THE SDF-1/CXCR4 AXIS in MYELOID SARCOMA}

*Annette H. Schmitt-Graeff ${ }^{1}$, Moritz Metzger ${ }^{1}$, Hartmut Bertz ${ }^{2}$, Michael Luebbert ${ }^{2}$, Jan A. Burger ${ }^{3}$

${ }^{1}$ Institute of Pathology and ${ }^{2}$ Dept. Hematology/Oncology, University Medical Center Freiburg, ${ }^{3}$ Department of Leukemia, the University of Texas MD Anderson Cancer Center, Houston, USA.
Background: The homing of hematopoietic stem/progenitor cells to distinct anatomic niches is mediated by the crosstalk between ligandreceptor signalling pathways. The relevance of chemokine-controlled recruitment of immature myeloid cells (IMC) to extramedullary sites resulting in myeloid sarcoma (MS) is largely unknown.

Purpose: We addressed the question of whether the lodgement of IMCs outside the bone marrow resulting in MS is mediated by the interaction between CXCR4 chemokine receptor, expressed on IMCs, and its ligand CXCL12 or stromal cell-derived factor-1 (SDF-1).

Methods: Clinico-pathological and survival data of 65 patients with a diagnosis of MS were analysed. Paraffin-embedded specimens containing representative formalin-fixed MS biopsy material were retrieved from our files. Tissue sections were stained for a large panel of antibodies including CD33, NPM1, CXCR4 and SDF-1. Imaging studies by confocal laser scanning microscopy focused on the spatial localization of stromal cells expressing SDF-1 and of CXCR4 expressing IMCs.

Results: MS developed either de novo without concomitant bone marrow involvement, simultaneously with bone marrow infiltration as initial manifestation of a myeloid neoplasm, or occurred subsequently to a previously diagnosed haematological disorder. Survival analyses showed no significant differences for the various subcategories of MS. However, the overall survival of patients treated with allogeneic bone marrow transplantation was significantly superior to those treated with conventional chemotherapy $(\mathrm{p}<0.005)$.

In contrast to the large variety of phenotypic profiles and clinical settings, a common feature of all samples was an increased density of SDF-1-positive stroma in close association with CXCR4 positive IMCs.

Conclusions: Our observations suggest that the SDF-1/CXCR4 axis is crucial for IMC homing to extramedullary sites. The pharmacological disruption of this axis may be a target of future therapeutic strategies in patients with MS. Actually, long term survival of patients with MS is only achieved by allogeneic bone marrow transplantation.

\section{BMS 29}

\section{LOW REPRODUCIBILITY OF MORHOLOGICAL FEATURES OF THE WHO CLASSIFICATION IN EARLY PRIMARY MYELOFIBROSIS.}

S.Koopmans ${ }^{*}$, F.Bot ${ }^{1}$, K.Lam ${ }^{2}$, A. van Marion ${ }^{1}$, J.Michiels ${ }^{3}$, H. de Raeve $^{3}$, K.Hebeda ${ }^{4}$

University Hospital Maastricht ${ }^{1}$, Rotterdam ${ }^{2}$, Antwerpen $^{3}$ and Nijmegen ${ }^{4}$, Belgium and The Netherlands

Background: The early phases of polycythaemia vera (PV), essential thrombocytosis (ET) and primary myelofibrosis (PMF) are difficult to distinguish as they share many morphological characteristics. The aim was to asses the reproducibility of these morphological hallmarks and to assess the ability to obtain a definitive diagnosis on selected trephine biopsy sections alone without knowledge of the clinical data. Methods: 44 subsequent trephines diagnosed as PMF grade 0/1 were retrieved from the files of the university hospital Antwerp, Belgium. They were blindly reviewed by 4 Dutch hematopathologists, using a standardized scoring system for the diagnosis based on 18 histological characteristics, mainly related to megakaryocyte (MK) morphology. Consensus was defined as agreement by 3 out of 4 pathologists. 
Results: a large variation in degree of consensus was apparent, varying from $95 \%$ for staghorn nuclei to as low as $42 \%$ for the grading of fibrosis. While in $100 \%$ the consensus diagnosis was MPN, only $74 \%$ consensus was reached for the specific diagnosis PMF, although all cases were previously classified as PMF according to combined clinical and morphological WHO criteria. PV was considered by one of the reviewers in 39\% and ET in 7\% of the cases.

\begin{tabular}{|c|c|c|c|c|c|}
\hline MK nuclei & $\%$ cons & clusters & $\%$ cons & $\begin{array}{l}\text { MK } \\
\text { cytoplasmn }\end{array}$ & $\%$ cons \\
\hline Staghorn & 95 & No & 75 & normal & 65 \\
\hline Cloud like & 85 & Loose & 79 & small & 92 \\
\hline Dysmorphic & 89 & Dense & 88 & large & 78 \\
\hline Bare nuclei & 88 & & & dysmorphic & 79 \\
\hline $\begin{array}{l}\text { Normal } \\
\text { lobulation }\end{array}$ & 97 & $\begin{array}{l}\text { Peritrabecular } \\
\text { fat }\end{array}$ & 92 & ME ratio & 72 \\
\hline Hyperlobulation & 86 & $\begin{array}{l}\text { Fibrosis } \\
\text { grading }\end{array}$ & 42 & & \\
\hline Hypolobulation & 82 & $\begin{array}{l}\text { Dilated } \\
\text { sinusoids }\end{array}$ & 82 & $\begin{array}{l}\text { MPN } \\
\text { subtype }\end{array}$ & 74 \\
\hline
\end{tabular}

Conclusion: Although the diagnosis of myeloproliferative neoplasm (MPN) was reproducibly made on selected trephine biopsies without knowledge of the clinical data, the consensus for the specific diagnosis was only $74 \%$ with a low degree of consensus for some major morphological hallmarks that define MPN. The reproducibility of the WHO morphological criteria appears at best fair for classification of early MPN or MPN with overlapping diagnostic features for the various subtypes.

\section{BMS 30}

\section{COMPARISON OF CD23 EXPRESSION} IN CHRONIC LYMPHOCYTIC LEUKEMIA (CLL) AND MANTLE CELL LYMPHOMA (MCL). CLINICAL UTILITY OF A THRESHOLD VALUE FOR EXPRESSION INTENSITY.

Ravi Patel ${ }^{2}$, Joanne Luider ${ }^{1}$, Xiu Jiang, Iwona Auer ${ }^{1}$, Meer-Taher Shabani-Rad ${ }^{1}$, Adnan Mansoor ${ }^{1}$.

Department of Pathology and Laboratory Medicine, University of Calgary \& Calgary Laboratory Services and ${ }^{2}$ MAster in Biotechnology program University of Calgary. Calgary, Alberta, Canada.

Objective: Most common differential diagnosis among CD19/CD5+ lymphoma is between CLL and MCL. WHO criteria identify the leukemic cells in CLL to be positive for CD19/CD5/CD23. Among MCL patients (pts) CD23 is either absent or weakly expressed. Thus mere positive and negative expression of $\mathrm{CD} 23$ fails to differentiate between CLL and MCL by flow-cytometry. The purpose of this study is to determine a threshold value for $\mathrm{CD} 23$ expression using flow cytometry, a value that can be adopted in clinical practice to differentiate between CLL and MCL.

Methods: Peripheral blood, bone marrow, lymph node tissue etc were stained with a 5-colour panel consisting of FMC7-FITC, CD43-PE, CD23-ECD, CD5-PC5 and CD19-PC7. Neoplastic cells were identified using a CD5/CD19 gate; median fluorescent intensities (MFI) as well as percentages of positive cells (\% positive) were recorded utilising FC500 flow-cytometer. Cyclin D1 and / or t $(11 ; 14)$ by FISH technique, confirmed diagnosis among MCL patients. 154 pts (131 CLL and 23 MCL) between the ages of 33-89 yrs (median 69yrs) were included. The samples comprised of peripheral blood $(108 ; 70 \%)$, bone marrow (34, 21\%), lymph node (7, 5\%) and others (5, 4\%). Using a MFI threshold of 0.700 to determine positivity, CD23 expression was able to differentiate between CLL and MCL (sensitivity of $96.1 \%$ \& specificity of $91.7 \% ; \mathrm{p}<0.0001)$. Similarly, a threshold of $40 \%$ to determine positivity, revealed a sensitivity of $94.7 \%$ and a specificity of $95.7 \%$. The Chi-square $\mathrm{p}$ value was $<0.0001$.

Conclusions: Increased CD23 expression with higher intensity is significantly more frequent in CLL when compared to MCL. Using a threshold of $40 \%$ positive, CD23 expression by flow-cytometry has significant predictive value in differentiating CLL from MCL.

\section{BMS 31}

\section{EXPRESSION OF VEGF, MCP-1 AND OSTEOPONTIN IN MYELOMA}

Sanja Štifter, Toni Valković ${ }^{1}$, Ksenija Lučin, Irena Seili-Bekafigo ${ }^{1}$, Antica Načinović-Duletić ${ }^{1}$, Blažen Marijić ${ }^{1}$, Merica Aralica ${ }^{2}$, Nives Jonjić

Department of Pathology, School of Medicine, University of Rijeka, Croatia ${ }^{1}$ Department of Hematology, Clinical Hospital Centre Rijeka, Croatia ${ }^{2}$ Clinical Laboratory, Clinical Hospital Centre Rijeka, Croatia

The role of angiogenesis in the pathogenesis of multiple myeloma (MM) is well recognized, however the involvement of myeloma cells and different microenvironment elements of bone marrow in this process is not completely recognized.

The aim of this study was to explore the expression of three different cytokines, acknowledged to be involved in the angiogenesis, at the protein level in bone marrow and in plasma of MM patients. The expression of cytokines was correlated with microvessel density (MVD) and clinicopathological characteristics of MM patients. Immunohistochemical analysis was performed in order to identify the vascular endothelial growth factor (VEGF), macrophage chemotactic protein-1 (MCP-1), osteopontin (OPN), and endothelial cells (CD34), while ELISA method was used for detection of VEGF, MCP-1 and OPN in plasma.

The results demonstrated higher MVD values in bone marrows with higher percentage of plasma cells infiltrates $(p<0.001)$; in patients with higher clinical stage $(\mathrm{p}=0.01)$; in no responders to therapy $(\mathrm{p}=$ $0.03)$, and in relation to shorter overall survival $(p=0.04)$. All three cytokines were detected at various percentage and staining intensity in the myeloma cells, different hematopoetic and stromal cells. Some positive staining was also observed in extracellular matrix of bone marrow. Association of all three cytokines expressed in the myeloma cells and in plasma of MM patients, was detected. The expression of VEGF and OPN in tumor cells demonstrated the inverse relationship with the MVD, while at the plasma level neither one of three analyzed cytokines showed correlation with MVD. Nevertheless, the plasma VEGF, MCP-1 and OPN concentrations were significantly higher in those patients with higher percentage of tumor cells infiltrates in bone marrow $(p<0.05)$. 
Present results confirmed the prognostic significance of MVD in MM. In addition, the association between VEGF/MCP/OPN expression level and tumor mass highlights their possible influence on myeloma pathogenesis, what should be further explored.

\section{BMS 32}

\section{IMMUNOHISTOCHEMICAL ANALYSIS OF YMPHOPLASMACYTIC LYMPHOMAS: CORRELATION OF CD138, VEGF, CYCLIN D1 EXPRESSION AND MICROVASCULAR CHARACTERISTICS WITH PATIENTS' CLINICAL DATA AND SURVIVAL.}

Levidou G. and Tzenou T., Kyrtsonis M.C., Korkolopoulou P., Kavantzas N., Androulaki A., Salpeas V., Vassilakopoulos T., Angelopoulou M., , Panayotidis P., Pangalis G.A., Patsouris E.

First Department of Propedeutic Internal Medicine, First Department of Internal Medicine, Department of Haematology and Department of Pathology, National and Kapodistrian University of Athens, Laikon University Hospital, Athens, Greece.

Background: Lymphoplasmacytic lymphomas (LPLs) are characterized by a clonal proliferation of neoplastic lymphocytes, lymphoplasmacytes and few plasma cells frequently infiltrating the bone marrow (BM).

Purpose of the study: BM paraffin embedded sections of 44 patients with newly diagnosed LPL, 32 of which fulfilled the criteria of Waldenstroms' Macroglobulinaemia were studied. We investigated the levels of cyclin D1, CD138 and VEGF expression as well as the microvascular parameters as evaluated by CD34 immunohistochemistry and image analysis in correlation with clinical features, patients' survival.

Results: $53 \%$ of cases displayed cyclin D1 immunoreactivity (range $1-70 \%$, median $=2 \%$ ), whereas all cases displayed CD138 (range 0$72 \%$ ) and VEGF (range $0.04-72 \%$ ) immunoreactivity. The percentages of VEGF and CD138 positive neoplastic cells were positively correlated with extend of BM infiltration $(p<0.0001$ and $p=0.0734$ respectively). CD138 expression increased in parallel with the levels of serum $\operatorname{IgM}(p=0.0111)$ and disease severity $(p=0.0263)$. VEGF expression was negatively correlated with platelet count $(\mathrm{p}=0.0409)$, was higher in patients with lymphadenopathy $(p=0.0178)$ and marginally increased in parallel with vascular area and feret diameter $(p=0.0639$ in both cases). Univariate survival analysis showed that patients with increased total vascular area (TVA) had a trend for lower probability of survival although this relationship failed to attain statistical significance $(p=0.0898)$.

Conclusions: We found a correlation CD138 expression in LPLs with the levels of serum $\operatorname{IgM}$, while there seems to be associations between VEGF and CDA138 expression in bone marrow infiltrating cells and clinical parameters. Importantly, despite the low number of patients enrolled TVA seems to be associated with patients' prognosis in LPLs. Further larger studies on this field are warranted in order to validate our results.

\section{Lymphoma Symposium 22 to 24 September 2008}

$\underline{\operatorname{LS} 1}$

\section{ASSESSMENT AND FUNCTIONAL ANALYSIS OF PAX5 MUTATIONS IN ADULTS B-CELL ACUTE LYMPHOBLASTIC LEUKEMIA/LYMPHOMA}

Marina Bousquet, Julien Familiades, Etienne Coyaud, Cathy Quelen, Georges Delsol, Nicole Dastugue, Eric Delabesse, Cyril Broccardo and Pierre Brousset

From INSERM U563 CPTP, CHU Purpan, Toulouse, Department of Pathology, 31059 Toulouse, Cedex, France

Background: $P A X 5$ is a transcription factor involved in B-cell differentiation. Rare translocations implicating PAX5 have been reported and extensively studied.

Purpose of the study: Following the description of a new $t(7 ; 9)(q 11$; p13) translocation in cases of B-cell acute lymphoblastic leukemia (BALL), we investigated a series of adult B-ALL for PAX5 abnormalities. A previous analysis of $\mathrm{PAX} 5 / \mathrm{X}$ chimeras based on chromatin immunoprecipitation and gene reporter assays showed that most of mutated proteins acted as transdominant negative on PAX5.

Results: Different novel transcriptions and PAX5 mutations were identified. In some cases, a loss of heterozigosity was disclosed. Since PAX5 is essential for B-cell differentiation, these translocations or mutations may account for the blockade of the leukemic cells at the pre B-cell stage. A screening of a large series of adult B-ALL (obtained from the French protocol GRAAL) showed that PAX5 mutations were specifically associated with the presence of Phi1.

Conclusion: Our results point out $P A X 5$ as a key player in the process of B-cell leukemogenesis. All these results will be discussed in the light recent literature in this field.

\section{LS 2}

\section{EXPRESSION OF MDM2 ONCOPROTEIN PREDICTS FOR POOR SURVIVAL IN DIFFUSE LARGE B-CELL LYMPHOMA (DLBCL) WITH WILD-TYPE TP53 GENE}

*KH Young, ${ }^{1} \mathrm{MB}$ Møller, ${ }^{2}$ GWB Colleoni, ${ }^{3}$ M Sánchez-Beato, ${ }^{4} \mathrm{TM}$ Green, ${ }^{2} \mathrm{~T}$ Thorborg, ${ }^{2}$ MA Piris, ${ }^{4}$ JC Eickhoff, ${ }^{1} \mathrm{M}$ Twohig, ${ }^{1} \mathrm{AH}$ Young, ${ }^{1}$ TD Oberley, ${ }^{1}$ JS Malter, ${ }^{1}$ JA Ferry, ${ }^{5}$ WC Chan, ${ }^{6}$ DD Weisenburger, ${ }^{6}$ and TC Greiner ${ }^{6}$

\footnotetext{
${ }^{1}$ Departments of Pathology and Laboratory Medicine, University of Wisconsin School of Medicine and Public Health, UW Paul P. Carbone Cancer Center, Madison, WI; ${ }^{2}$ Odense University Hospital, Odense, Denmark; ${ }^{3}$ Federal University of São Paulo, Brazil; ${ }^{4}$ Spanish National Cancer Center, CNIO, Madrid, Spain; ${ }^{5}$ Harvard Medical School, Boston, MI; ${ }^{6}$ University of Nebraska Medical Center, Omaha, NE
} 
Background: The MDM2 gene encodes for a nuclear phosphoprotein, and its expression has been implicated in the pathogenesis of human neoplasms via inhibition of the p53 tumor-suppressor pathway.

Purpose of the study: The purpose of this study is to investigate the potential role of MDM2 protein by correlating the expression of MDM2 with TP53 mutation status in DLBCL. The TP53 gene was analyzed for mutations and immunostains for $\mathrm{p} 53$ and MDM2 protein expression were performed in 133 cases from 6 medical centers. A positive immunostain was defined as nuclear staining in $10 \%$ or more of the tumor cells. The Kaplan-Meier method was used for survival analysis.

Summarized description of the results: MDM2 stains were positive in 64 of 133 cases of DLBCL (48\%). While there was a suggestion of poor overall survival (OS) with MDM2 expression, it was not statistically significant in the entire group of DLBCL. Twelve of 24 cases $(50 \%)$ with TP53 mutations had MDM2 expression. But no significant difference in OS was observed between MDM2-positive and MDM2-negative cases in this TP53 mutated group $(p=0.32)$. Similarly, 51 of 107 cases (48\%) with wild-type (WT) TP53 gene had MDM2 expression. But in this group, the MDM2-positive phenotype predicted for poor 5 -year OS $(38 \%$ vs $67 \%, p=0.002)$, and a significantly-shorter median disease-free survival $(2.3$ years $)$ as compared to those without MDM2 expression ( $>5.0$ years; $p=$ 0.013 ). The complete remission rate was only $57 \%$ in MDM2-positive cases compared to $73 \%$ in MDM2-negative cases in the WT-TP53 group $(\mathrm{p}=0.08)$. Multivariate analysis confirmed that MDM2 expression was an independent predictor of poor OS in DLBCL with a WT-TP53 gene (HR 2.0, 95\% CI 1.15-3.56; $\mathrm{p}=0.015$ ).

Conclusion: This study demonstrates the importance of MDM2 expression in predicting survival in patients with DLBCL, representing an alternative mechanism of $\mathrm{p} 53$ pathway inactivation in cases with a WT-TP53 gene.

\section{$\underline{\text { LS } 3}$}

\section{PERFORATION BUT NOT IMMUNOPHENOTYPE PREDICTS POOR PROGNOSIS IN PATIENTS WITH PRIMARY INTESTINAL DIFFUSE LARGE B-CELL LYMPHOMA}

*Shih-Sung Chuang, ${ }^{1}$ Sheau-Fang Yang, ${ }^{2}$ Wan-Ting Huang, ${ }^{3}$ Pin-Pen $\mathrm{Hsieh}^{4}$

Department of Pathology, ${ }^{1}$ Chi-Mei Medical Center, Tainan; ${ }^{2}$ Kaohsiung Medical University Hospital, Kaohsiung; ${ }^{3}$ Chang Gung Memorial Hospital, Kaohsiung; ${ }^{4}$ Veterans General Hospital, Kaohsiung, Taiwan.

Background: Most primary gastrointestinal lymphomas occur in the stomach and less commonly in the intestine, with mucosa-associated lymphoid tissue lymphoma and diffuse large B-cell lymphoma (DLBL) as the most frequent histological types. There are very limited data on the prognostic factors of primary intestinal DLBL, and the survival impact of germinal center B-cell (GCB) vs. non-GCB phenotype has not studied.

Purpose of the study: The purpose of this retrospective study was to identify the prognostic factors including perforation and cell differentiation antigens in primary intestinal diffuse large B-cell lymphoma (PI-DLBL).
Methods and results: Archival tissues from 30 tumors were used for tissue microarray construction and immunohistochemical study. The M: F ratio was 1.7: 1 with a median age of 60 years old. Ileum and Ileocecum were most frequently involved, with $12(40 \%)$ cases each in both regions. Fourteen (47\%) were at stage IE disease; $15(50 \%)$, stage IIE. Five $(17 \%)$ tumors were perforated at presentation. The tumors expressed bcl-6 (73\%), MUM1 (21, 70\%), bcl-2 (67\%), and CD10 (23\%). Nine (30\%) were classified as GCB phenotype and 21 , non-GCB. Perforation was a poor prognostic indicator with a hazard ratio of tumor-related death at $8.75(P=.001)$. All the antigens $(\mathrm{CD} 10$, bcl-2, bcl-6, MUM1, and Ki-67 labeling index) tested and GCB vs. non-GCB phenotype did not carry any prognostic significance.

Conclusions: We found a higher rate of perforation and lower frequency of GCB phenotype in PI-DLBL in Taiwan as compared to other geographic areas. Furthermore, perforation is a poor indicator of survival but not the differentiation antigens or GCB vs. non-GCB phenotypes.

\section{$\underline{\operatorname{LS} 4}$}

\section{THE PHENOTYPE OF INTRAEPITHELIAL LYMPHOCYTES IN TAIWANESE ENTEROPATHY- ASSOCIATED T-CELL LYMPHOMA IS DISTINCT FROM THAT OF THE WEST}

*Shih-Sung Chuang ${ }^{1}$, Yung-Liang Liao ${ }^{1}$, Hoxiang $\mathrm{Liu}^{2}$, Pin-Pen Shieh $^{3}$, Wang-Tin Huang ${ }^{4}$, Han-Ku Chen ${ }^{4}$, Peter G. Isaacson ${ }^{5}$

${ }^{1}$ Department of Pathology, Chi-Mei Medical Center, Tainan, Taiwan ${ }^{2}$ Department of Histopathology, Addenbrooke's Hospital, Cambridge, U.K.; ${ }^{3}$ Department of Pathology, Veterans General Hospital-Kaohsiung, Kaohsiung, Taiwan; ${ }^{4}$ Department of Pathology, Chang Gung Memorial Hospital - Kaohsiung Medical Center, Taiwan; ${ }^{5}$ Department of Pathology, University College London, London, U.K.

Background: Enteropathy-associated T-cell lymphoma (EATL) is a rare complication of celiac disease (CD) with intraepithelial lymphocytes (IELs) as the cellular origin of neoplastic cells.

Purpose of the study: To characterize EATL in Taiwan. Results: We collected 19 primary intestinal $\mathrm{T}$ and NK/T-cell lymphomas in three medical centers in Taiwan including 15 peripheral T-cell lymphomas, unspecified, and two cases each of extranodal NK/T-cell lymphoma and EATL. In the two cases of EATL, there was massive infiltration of IELs in the mucosa away from the tumor proper. The neoplastic cells in the tumor proper expressed CD3, CD7, CD8, CD56, and TIA-1, but not CD4, CD5, CD20, or CD30. The phenotype of the IELs was different from that of the tumor proper: the IELs in both cases lost CD8 and CD56, and in addition, the IELs in Case 2 lost CD2 and granzyme B. Both tumors were negative for EBER. The tumor cells in Case 1 were oligoclonal, and the same-sized bands were present in the microdissected IEL part. The tumor cells in Case 2 were clonal, and the same-sized bands were identified in the IEL part.

Conclusions: The two EATL cases were different from the Western type B EATL in that the phenotype of the neoplastic cells in the tumor proper and IELs was distinct, in contrast to the shared phenotype in the Western EATL cases. Although reason for this discrepancy is unknown, this is evidence that EATL in Taiwan, where CD has never been reported, is not CD-related. We suspect that these two cases are mere morphologic mimics of the rare Western type B EATL but not a complication of CD. More cases with serum and fresh tumor tissue are needed for clarification of the relationship between CD and EATL in Taiwan. 


\section{LS 5}

\section{PATHOLOGICAL AND IMMUNOHISTOCHEMICAL STUDY OF GASTROINTESTINAL LYMPHOMAS WITH DETECTION OF CHROMOSOMAL TRANSLOCATION T(11;18) BY FLUORESCENCE IN SITU HYBRIDIZATION}

Iman Talaat; Assistant Lecturer of Pathology, Amany Serour; Assistant Professor of Clinical Pathology, Nagwa Mashali*; Professor of Pathology*, Samar El Sheikh; Assistant Professor of Pathology, Alexandria Faculty of Medicine, Egypt.

Background: Gastrointestinal lymphomas are the most common extra-nodal lymphomas accounting for about $40 \%$ of extra-nodal lymphomas. The stomach is the most common location, followed by the small intestine, and rarely the colon.

MALT lymphomas are a subgroup of low-grade B-cell lymphomas that arise from extra-nodal sites that have accumulated MALT as a result of a chronic inflammatory disorder. Recently, the application of techniques such as FISH has improved the characterization of MALT lymphomas at the genetic level.

Aim: To study GIT lymphomas pathologically and to detect the presence of translocation $\mathrm{t}(11 ; 18)(\mathrm{q} 21 ; \mathrm{q} 21)$ in these tumors by FISH technique.

Material: The present study was conducted on 27 specimens diagnosed as primary GIT-NHL. These specimens were selected from a total of 57 archived specimens with the same diagnosis.

Methods: Pathological evaluation of the specimens (gross and microscopic description), followed by the application of FISH on paraffin-embedded thin sections using LSI API2/MALT1 $t(11 ; 18)$ (q21;q21) dual color dual fusion translocation probe.

Results: Six out of 27 studied cases (22.2\%) gave positive scoring of $\mathrm{t}$ $(11 ; 18)(\mathrm{q} 21 ; \mathrm{q} 21)$ by FISH. All positive cases proved to be MALT lymphoma with no concomitant areas of diffuse large B cell lymphoma. Five out of the six cases (83.3\%) had gastric localization, whereas only one case was located in the small intestine.

Conclusion: The present study emphasized the successful application of FISH technique on paraffin embedded sections, thus allowing its application on retrospective cases.

\section{LS 6}

\section{SIGNIFICANT INCREASE IN THE RELATIVE FREQUENCY OF FOLLICULAR LYMPHOMA IN TAIWAN IN THE EARLY 21ST CENTURY}

*Shih-Sung Chuang, M.D.

Department of Pathology, Chi-Mei Medical Center, Tainan, Taiwan

Purpose of the study: To clarify the impression of increased incidence of follicular lymphoma (FL) in routine diagnostic practice. Methods: The author analyzed the lymphoma cases diagnosed from Jan 2005 to Dec 2007 at Chi-Mei Medical Center and compared the results with the data published in 1989-1998 from the same institution (Cancer 2000; 89: 1586). All consultation cases to the author and a single non-Taiwanese patient were excluded.
Results: Among the 279 in-house Taiwanese cases, 39 cases were excluded either because they were diagnosed at other hospitals and immediately referred for treatment (18 patients) or came for relapsed diseases after initial diagnosis and treatment at other hospitals (21 patients). Of the 240 lymphomas, 12 (5.0\%) were classical Hodgkin lymphomas and $228(95.0 \%)$ were non-Hodgkin's lymphomas (NHL). Among NHL, the relative frequency of FL increased significantly from $6.1 \%$ to $14.5 \%(p=.005)$ and became the second most common subtype after diffuse large B-cell lymphoma. The other significant changes included decreased frequency of mucosa-associated lymphoid tissue lymphoma (from $19.7 \%$ to $7.9 \% ; p=.0001$ ) and increased incidence of small lymphocytic lymphoma/chronic lymphocytic lymphoma (from $1.0 \%$ to $7.0 \% ; p=.002$ ).

Conclusions: The relative frequency of FL in Taiwan has been increasing in the past years toward the higher rate as seen in the West, maybe reflecting the changing life style and socio-economic conditions of Taiwan into a developed country. Epidemiological studies and analytical studies such as case-control/cohort studies are needed to resolve this issue. Furthermore, molecular studies comparing recent cases and those of one to two decades ago with probes directing at both the $M B R$ and $m c r$ regions of $B C L-2$ translocation might help elucidating the molecular mechanisms of FL in Taiwan.

\section{LS 7}

\section{HIGH NUMBERS OF TUMOR INFILTRATING PD-1-POSITIVE REGULATORY LYMPHOCYTES ARE ASSOCIATED WITH IMPROVED OVERALL SURVIVAL IN FOLLICULAR LYMPHOMA}

*Joaquim Carreras ${ }^{1,3}$, Armando Lopez-Guillermo², Giovanna Roncador $^{4}$, Neus Villamor ${ }^{1}$, Lluis Colomo ${ }^{1}$, Antonio Martinez ${ }^{1}$, Rifat Hamoudi $^{3}$, Will Howat ${ }^{5}$, Emili Montserrat ${ }^{2}$, Elias Campo ${ }^{1}$.

${ }^{1}$ Hematopathology Section, Departments of Pathology, and ${ }^{2}$ Hematology, Hospital Clinic, IDIBAPS, University of Barcelona, 08036-Barcelona, Spain. ${ }^{3}$ Division of Molecular Histopathology, Department of Pathology, University of Cambridge, Cambridge, UK. ${ }^{4}$ Centro Nacional de Investigaciones Oncológicas (CNIO), Madrid, Spain. ${ }^{5}$ Histopathology/ISH facility, Cancer Research UK, Cambridge Research Institute. Li Ka Shing Centre, Cambridge, UK.

Purpose: Tumor microenvironment influences the behavior of follicular lymphoma (FL), although the specific cell subsets involved are not well known yet. The aim of this study was to determine the impact of programmed cell death 1 (PD-1) positive inhibitory immunoregulatory lymphoid cells in the clinicobiological features and outcome of patients with FL.

Patients and methods: We examined samples from 100 patients $(53 \mathrm{M} / 47 \mathrm{~F}$; median age 54 years) at diagnosis, as well as in 32 patients at first relapse, with a recently generated monoclonal antibody against PD-1. The cells were quantified using computerized image analysis. Additional analysis consisted in double immunofluorescence and flow cytometry.

Results: PD-1 expression was alternative to FOXP3 in lymphoid cells from both reactive tonsils and FL. At diagnosis, the median percentage of PD-1-positive cells was 14\% (range, 0.1-74). Patients with grade $3 \mathrm{FL}$, poor performance status and high serum LDH showed lower numbers of PD-1-positive cells. After a median followup of 6.2 years, patients with PD-1-positive cells $\leq 5 \%(\mathrm{~N}=25), 6-33 \%$ 
$(\mathrm{N}=50)$ and $>33 \%(\mathrm{~N}=25)$ had a 5 -year progression-free-survival of 20,46 and $48 \%(p=0.038)$, and OS of 50,77 and $95 \%(p=0.004)$, respectively. PD-1 and FLIPI maintained prognostic value for OS in multivariate analysis. Patients with PD-1-positive cells $\leq 5 \%$ showed a higher risk of histological transformation. At that time, transformed diffuse large B-cell lymphomas (DLBCL) had lower percentage of PD-1-positive cells than FL.

Conclusion: a high content of PD-1-positive cells predicted favourable outcome of FL patients, while a marked reduction is observed in transformation.

\section{LS 8}

\section{SOMATIC HYPERMUTATION OF Ig $V_{H}$ GENES AND ABERRANT SOMATIC HYPERMUTATION IN BCL-2 NEGATIVE FLs}

*É. Gagyi, Zs. Balogh, Cs. Bödör, B. Timár, L. Reiniger, L. Deák, J. Csomor, B. Csernus, Á. Szepesi and A. Matolcsy

1st Department of Pathology and Experimental Cancer Research, Faculty of Medicine, Semmelweis University, Budapest, Hungary

Follicular lymphoma (FL) is characterized by the $\mathrm{t}(14 ; 18)$ translocation resulting in the constitutive expression of BCL-2 protein, however approximately $10-15 \%$ of FLs are negative for BCL-2 protein, and a small fraction of these cases does not exhibit the translocation of the $B c l-2$ gene either. It is highly debated whether FLs without $\mathrm{Bcl}-2$ gene rearrangement and expression represent a separate lymphoma entity with distinct biological characteristics, different from BCL-2 positive FLs. In this entity the role of somatic hypermutation (SHM) in the diversification of immunoglobulin genes and the malfunction of the physiological SHM, termed aberrant somatic hypermutation (ASHM), which affects additional genes such as Pax-5, RhoH/TTF, c-Myc, Piml, is not explored. To further characterize $\mathrm{FLs}$ without $\mathrm{Bcl}-2$ gene rearrangement and expression, we analyzed the mutational status of $\lg V_{H}$ genes as well as certain other genes (c-Myc, Pax-5 and RhoH) frequently involved in the specific type of genomic instability called ASHM in 11 cases of BCL2 negative FLs. We also determined the activation-induced cytidine deaminase (AID) expression levels in these lymphomas. Our findings demonstrate that FLs without $\mathrm{Bcl}-2$ gene rearrangement and expression are associated with ongoing type of somatic hypermutation (SHM) of the $\operatorname{Ig} V_{H}$ genes, low activity of ASHM and elevated AID expression. These results indicate that similar to BCL-2 positive FLs, BCL -2 negative FLs are of germinal center B-cell origin, and can be considered as a single entity with many common molecular features.

\section{LS 9}

\section{PERIPHERAL T-CELL LYMPHOMA (PTCL) WITH LARGE B-CELLS (LBCS) - 30 CASES IN SINGAPORE}

*LHC Tan ${ }^{1}$, LL Chiu ${ }^{2}$, AKW Lee ${ }^{1}$ and ESC Koay ${ }^{2,3} .{ }^{1}$ Department of Pathology, Singapore General Hospital, Singapore; ${ }^{2}$ Department of Laboratory Medicine, National University Hospital, Singapore, Singapore and ${ }^{3}$ Department of Pathology, National University of Singapore, Singapore.

Background: In PTCLs, functional impairment of immunosurveillance upon neoplastic transformation of T-lymphocytes may allow secondary proliferation of LBCs that are often EBV-driven and sometimes ReedSternberg-like, creating diagnostic confusion.

Aim: To characterise 30 of such cases by immunomorphology and molecular studies.

Results: The mean patient age was 58.9 (range 27-88) years, with a male:female ratio of $2: 1 ; 77 \%$ were Chinese; $87 \%$ had nodal presentation. $57 \%$ were initially not correctly diagnosed, $23 \%$ being labelled "atypical lymphoid hyperplasia". 19/29 (65.5\%) yielded clonal TCR gene rearrangements. 24/28 (85.7\%) were CD4+, and $15 / 24(62.5 \%)$ had EBV+ LBCs.

Only 14 (47\%) were angioimmunoblastic (AITL), 2 (7\%) with synchronous $T C R$ and $I g H$ clonality, the latter transient in 1 case (also the one of 3 with hyperplastic germinal centres). One case each of AITL recurred as either LBC lymphoma (LBCL) or classical Hodgkin lymphoma (cHL), neither with initially demonstrable $I g H$ clonality.

Three cases (10\%) amounted to composite unspecified PTCL-cHL, but an additional $4(15 \%)$ were originally misinterpreted as HL, including 1 involving marrow with a discordant nodal diagnosis of LBCL. Two other marrow cases showed either spontaneous disappearance of $\mathrm{LBC}$ or phenotypic change from $\mathrm{CD} 20+/ \mathrm{CD} 30$ - to CD20-/CD30+, clinching the primary pathology as PTCL despite lack of demonstrable TCR clonality. Another case (AITL), with TCR monoclonality but $I g H$ polyclonality, disclosed transient plasmacellular lambda light chain restriction in marrow.

Conclusion: PTCLs with LBCs are more often of unspecified type than AITL. The LBCs may spontaneously disappear, undergo phenotypic modulation, or recur as LBCL or cHL. $I g H$ clonality may not portend recurrence as LBCL.

\section{LS 10}

\section{LYMPHOPROLIFERATIVE DISORDERS WITH GENOTYPE-PHENOTYPE DISCORDANCE}

\footnotetext{
*LHC Tan ${ }^{1}$, ST $\mathrm{Lim}^{2}$, CTH Chuah ${ }^{3}, \mathrm{M} \mathrm{Tao}^{2}$ and ESC Koay ${ }^{4}$.

${ }^{1}$ Department of Pathology, Singapore General Hospital, Singapore; ${ }^{2}$ Department of Medical Oncology, National Cancer Centre, Singapore; ${ }^{3}$ Department of Haematology, Singapore General Hospital, Singapore and ${ }^{4}$ Department of Pathology, National University of Singapore, Singapore.
} 
Background: Antigen receptor PCR analysis may yield a clonal genotype of lineage discordant from that expected from histology and immunophenotype.

Aim: To characterise 7 of such cases by immunomorphology and molecular studies.

Results: Five B-cell lymphomas (BCLs) showed discordant TCR clonality [a marginal zone lymphoma (MZL) each of: (1) liver (L) with antimitochondrial antibody (AMA)+ primary biliary cirrhosis (PBC), (2) colon with associated H. pylori $(\mathrm{Hp})$ gastritis, and (3) marrow (M); (4) CLL with cutaneous vasculitis, (5) EBV+ large BCL [LBCL] in a HIV+ patient], while (6) a marrow showed transient lambda-restricted plasma cells (mimicking Case 3 ) associated with Tcell lymphoma (TCL), and (7) a nasal-type (EBV+) NK lymphoma presented with transient $\mathrm{IgH}$ monoclonality in aqueous humour. On follow-up, Case 1 showed cutaneous anaplastic large T-cell lymphoma of clonotype identical to that in the AMA+ PBC 9 years before, while in Case 3, anti-CD20 treatment unmasked an underlying TCL with an aberrant CD2/4/5+,CD3/7/8- phenotype.

Conclusion: Transient, non-neoplastic antigen receptor gene clonality may be detected in the setting of autoimmunity, chronic (especially EBV and Hp) infection, or immunodeficiency (HIV and TCL), implying underlying immune dysregulation and/or superantigen effect with resultant antigen receptor repertoire restriction. Furthermore, concordant clonality may not be detected in MZL and HIV/EBV+ LBCL (due to $\operatorname{IgH}$ somatic mutations), as well as NK lymphoma (which has germline $T C R$ and $I g H$ genes). However, persistent clonotype discordance may reflect a second lymphoma, particularly a TCL, since immunodeficiency due to neoplastic transformation of Tcells may have drawn clinical attention in the first place.

\section{$\underline{\text { LS } 11}$}

\section{EXPRESSION AND ABERRATION OF P16 GENE IN DIFFUSE LARGE B-CELL LYMPHOMA}

\author{
Lee Ee-Soo ${ }^{a}$, Kim Lian-Hua ${ }^{b}$, *Peh Suat-Cheng ${ }^{a}$ \\ ${ }^{a}$ Department of Pathology, Faculty of Medicine, University of \\ Malaya, Kuala Lumpur, Malaysia. b Department of Bioscience, \\ Faculty of Engineering and Science, University of Tunku Abdul \\ Rahman, Kuala Lumpur, Malaysia.
}

Aberration of p16 gene may cause cell cycle disruption that allows abnormal proliferation of cancer cells. Deletion of p16 region on chromosome 9p21 is a potential inactivation mechanism of p16 gene. There are some studies which revealed that gain of $9 p$ chromosomal arm and/or chromosomal materials of 9 p21 were common in haematologic malignancies. This study was undertaken to investigate expression of p16 protein and abnormalities of the gene in diffuse large B-cell lymphoma (DLBCL). Immunohistochemical expression of p16 was evaluated in 70 paraffin-embedded DLBCL tissues. Four cases were non-reactive for p16 staining and excluded from statistical analysis. Increased expression of p16 was detected in 43.9\% $(29 / 66)$ of all the cases successfully studied. We found that increased expression of p16 was more common in germinal centre B-cell-like (GC) $(60 \%, 15 / 25)$ compared to non-GC $(34.1 \%, 14 / 41)$ subgroups. Therefore, there is significant association between elevation of $\mathrm{p} 16$ immunoreactivity and prognostic subgroups of DLBCL $(P=0.04)$.
Fluorescence in situ hybridization (FISH) was employed to further characterise status of p16 gene in 32 DLBCL cases. FISH was successfully performed on 24 of 32 cases, 8 cases showed low-signal intensity and/or insufficient material for comprehensive FISH studies, thus they were excluded from the analysis. Hemizygous and/or homozygous deletion of p16 gene were identified in 55.6\% (5/9) and $66.7 \%(10 / 15)$ of GC and non-GC cases, respectively, whereas gain of $9 \mathrm{p}$ chromosomal arm and/or chromosomal materials of $\mathrm{p} 16$ gene were shown in $88.9 \%(8 / 9)$ and $86.7 \%(13 / 15)$ of GC and non$\mathrm{GC}$ cases, respectively. In conclusion, increased expression of $\mathrm{p} 16$ is more frequent in GC subgroup compared to non-GC subgroup. More FISH analysis would be performed to determine the correlation of increased expression of p16 protein and abnormalities of the gene.

\section{$\underline{\operatorname{LS} 12}$}

\section{PRIMARY CUTANEOUS CYTOTOXIC T-CELL LYMPHOMAS: A MORPHOLOGICAL, IMMUNOPHENOTYPIC, AND MOLECULAR STUDY OF 35 PATIENTS}

Hagiwara Masahiro

Department of Dermatology, Nagoya Graduate School

Abstract: The objective of our study was to investigate the clinicopathological features of currently ill-defined subtypes of primary cutaneous cytotoxic T-cell lymphoma (CTCL), including: Epstein-Barr Virus (EBV)-positive CTCL of unspecified type (CTCLU) $(n=3)$, anaplastic large cell lymphoma (ALCL) $(n=5)$, and EBV-negative CTCLU $(n=27)$. EBV-positive CTCLU was characterized by aggressive behavior similar to that observed in nasal type NK/T cell lymphoma. Cutaneous ALCL had an indolent clinical course and patients survived through the follow-up examination. Patients with EBV-negative CTCLU had intermediate prognoses between those of patients with EBV-positive CTCLU and ALCL. Notably, the rates of spontaneous regression were relatively similar in ALCL (40\%) and EBV-negative CTCLU (26\%). We divided the latter group into the following 4 subcategories: (a) epidermotropic CD8+ Tcell lymphoma $(n=5)$, (b) cutaneous $\gamma / \delta$ T-cell lymphoma $(n=8),(c)$ cutaneous $\alpha / \beta$ pleomorphic T-cell lymphoma $(n=8)$, and $(d)$ cutaneous medium/large pleomorphic T-cell lymphoma, not otherwise specified $(n=6)$. All four of these groups of lymphomas exhibited a relatively favorable clinical course compared to previous reports. However, epidermotropic CD8+ T-cell lymphoma appeared to be unique with a higher ratio $(80 \%)$ of spontaneous regression, a lower ratio $(40 \%)$ of subcutaneous involvement, and a more favorable clinical course than the other three subcategories. These EBV-negative CTCLU cases were further subdivided into three clinical subgroups, as follows: Subgroup A (survival with partial or complete spontaneous resolution), Subgroup B (survival without spontaneous resolution), and Subgroup C (a fatal clinical course after diagnosis). Subgroup A was exclusively male, highly positive for TCR $\beta$ and $\mathrm{CD} 8$, negative for CD56 and TCR gene rearrangements, had few poorer clinical parameters, and appeared to be distinct from the other Subgroups B and C. IPI and PIT scores were useful markers for distinguishing between Subgroups B and C without any differences in the immunophenotypic findings. 
LS 13

\section{BURKITT LYMPHOMA IN UGANDA IN THE THIRD MILLENNIUM: A MORPHOLOGIC AND MOLECULAR APPRAISAL ON TISSUE MICRO-ARRAY.}

${ }^{(*)}$ Cristina Campidelli MD ${ }^{1}$, Lynnette K. Tumwine $\mathrm{MD}^{2}$, Simona Righi BS ${ }^{1}$, Stefano A. Pileri MD ${ }^{1}$

${ }^{1}$ Chair of Pathology, Unit of Hematopathology, Institute of Hematology and Clinical Oncology "L. \& A. Seràgnoli", Bologna University School of Medicine, 40138 Bologna, Italy ${ }^{2}$ Department of Pathology, Makerere University Medical School, P.O. Box 7072, Kampala, Uganda

Ninety-five endemic Burkitt lymphoma (BL) cases, collected at Makerere University over 10 years, were studied by the tissue micro-array (TMA) technology. The sites of disease involvement were the abdomen (57\%), lymph node (33\%), and jaw $(10 \%)$. Morphologically, 43 cases exhibited overt plasmacytoid differentiation as usually seen in HIV-positive patients in Western Countries. All cases turned out to be $\mathrm{CD} 10+, \mathrm{Bcl}-6+, \mathrm{Bcl}-2-$ and $\mathrm{EBV}+$, with a. proliferation index close to $100 \%$. Interestingly, CD30 and CD138 expression was recorded in 35 and 43 instances, respectively. The former finding largely corresponded to the plasmacytoid differentiation observed at microscopic evaluation. On the contrary, the latter was detected irrespectively of morphology. The prevalence of $t(8 ; 14)$ could not be assessed due to DNA degradation. Therefore, the determination of CD38, CD44 and TCL1 was performed as a potential surrogate of the molecular test accordingly to Rodig et al. (Am J Surg Pathol 2008; 32:113-22). Out of 85 evaluable cases 73 displayed an antigen combination consistent with the presence of $t(8 ; 14)$. Finally, HIV-infection was searched by PCR and probes spanning the short gag and env regions. None of 95 cases tested showed HIV integration.

Our study displays the following novel features:

1) the jaw was much less frequently affected than expected, this probably meaning that BL does nowadays occur in a slightly older population than in the past;

2) plasmacytoid differentiation is also frequently observed in Central Africa, as further supported by the CD138 staining;

3) such differentiation is not related to HIV-infection, but else seems to reflect the multi-step lymphomagenesis typical of BL (EBV, malaria, Arboviruses, and Euphorbia Tirucalli);

4) CD30 expression can be related to the latter process;

$\mathrm{t}(8 ; 14)$ might occur not in all BL cases as recently suggested by Leoncini et al. (International Conference on $\mathrm{BL}$ and related lymphoproliferative disorders; Kampala, February 25-27, 2008).
LS 14

\section{DETECTION OF GENOMIC IMBALANCES IN MICRODISSECTED PRIMARY HODGKIN- AND REED-STERNBERG CELLS BY OLIGONUCLEOTIDE BASED ARRAY COMPARATIVE GENOMIC HYBRIDIZATION}

*Sylvia Hartmann ${ }^{1}$, José I. Martin-Subero ${ }^{2}$, Stefan Gesk $^{2}$, Julia Hüsken ${ }^{1}$, Maciej Giefing ${ }^{2,3}$, Inga Nagel ${ }^{2}$, Jennifer Riemke ${ }^{2}$, Andreas Chott $^{4}$, Wolfram Klapper ${ }^{5}$, Marie Parrens ${ }^{6}$, Jean-Philippe Merlio ${ }^{6}$, Ralf Küppers ${ }^{7}$, Andreas Bräuninger ${ }^{1}$, Reiner Siebert ${ }^{2}$, Martin-Leo Hansmann ${ }^{1}$

${ }^{1}$ Senckenberg Institute of Pathology, University of Frankfurt, Theodor-Stern-Kai 7, 60590 Frankfurt, Germany ${ }^{2}$ Institute of Human Genetics, Christian-Albrechts-University Kiel, University Hospital Schleswig- Holstein, Campus Kiel, 24105 Kiel, Germany ${ }^{3}$ Institute of Human Genetics, Polish Academy of Sciences, 60-479 Poznan, Poland ${ }^{4}$ Department of Pathology, Medical University of Vienna, Vienna, Austria ${ }^{5}$ Department of Pathology, Christian-AlbrechtsUniversity Kiel, University Hospital Schleswig- Holstein, Campus Kiel, 24105 Kiel, Germany ${ }^{6}$ Department of Pathology and Tumour Genetics, CHU Bordeaux and EA2406 University of Bordeaux 2, Bordeaux, France ${ }^{7}$ Institute for Cell Biology (Tumor Research), University of Duisburg-Essen, Medical School, Essen, Germany

Due to the usually low content of Hodgkin- and Reed-Sternberg (HRS) cells in classical Hodgkin lymphoma (HL), analysis of primary HRS cells remains difficult. They cells have been investigated by comparative genomic hybridization (CGH) to metaphases. Since DNA from microdissected HRS cells usually has to be amplified, an amplification bias may be introduced which can be detected by array CGH. In order to avoid DNA amplification, 10 HRS cell rich HL cases (nodular sclerosis/ lymphocyte depleted HL) were selected for this study. DNA obtained from approximately 100,000 microdissected HRS cells per case was analyzed on 105K CGH arrays containing 105,000 oligonucleotide probes. Aberrations of selected genes were confirmed by fluorescence in situ hybridization (FISH) and FICTION in the same HL cases and in a new series of HL cases with typical HRS cell content.

Recurrent gains of regions of chromosome arms $2 p$ and $9 p$ as well as losses of chromosome $13 \mathrm{q}$ and $\mathrm{X}$, known from metaphase $\mathrm{CGH}$, were confirmed by array CGH. In addition hitherto unknown gained and lost regions could be identified. Gained regions included several genes constitutively expressed in HL (e.g. STAT6, NOTCH1, TRAF2, RELA, CCND1, RELB, JUNB, NFkB2, E2F1). Gains of STAT6, NOTCH1 and JUNB were also detected recurrently by FISH in HL cases with low HRS cell content. The smallest deletion detected by array CGH and confirmed by multiplex quantitative PCR measured $156 \mathrm{~kb}$ containing the tumor suppressor gene CDKN2B.

The present study shows that array CGH of primary HRS cells without any amplification method allows the detection of new and the delineation of known genomic imbalances. Detection of imbalances of $156 \mathrm{~kb}$ size offers a much higher resolution than obtained by metaphase CGH. Genomic aberrations include some genes constitutively expressed in HL, possibly due to a gene dosage effect in rare cases. 
LS 15

\section{THE PROGNOSTIC VALUE OF THE TUMOR/ MICROENVIRONMENT SIGNATURE COMPARED TO EARLY PET SCAN IN ADVANCED-STAGE, ABVD-TREATED CLASSICAL HODGKIN LYMPHOMA (CHL) PATIENTS.}

${ }^{1 *} \mathrm{C}$ Agostinelli, ${ }^{2 \#} \mathrm{~A}$. Gallamini, ${ }^{\#} \mathrm{~L}$ Rigacci, ${ }^{\circ} \mathrm{F}$. D'Amore, ${ }^{\#} \mathrm{~F}$ Merli, ${ }^{\#}$ PL Zinzani, ${ }^{\#} \mathrm{U}$ Vitolo, ${ }^{\#} \mathrm{C}$ Patti, ${ }^{\#} \mathrm{C}$ Stelitano, ${ }^{\#} \mathrm{~F}$ Di Raimondo, ${ }^{\#} \mathrm{~A}$ Levis, ${ }^{\#} \mathrm{~L}$. Trentin, ${ }^{\#} \mathrm{~T}$ Chisesi ${ }^{\circ} \mathrm{P}$ Kamper, ${ }^{\#} \mathrm{P}$ Torchio, ${ }^{1} \mathrm{MT}$ Sista and ${ }^{1} \mathrm{~S}$. Pileri, on behalf of ${ }^{\#}$ Intergruppo Italiano Linfomi and ${ }^{\circ}$ Danish lymphoma group.

${ }^{1}$ Hemopathology Unit, Bologna University, Bologna, Italy. ${ }^{2}$ Hematology Department, S. Croce Hospital, Cuneo, Italy.

Gallamini et al. (JCO 2007) reported that early-interim PET-scan is the only tool predicting treatment outcome in advanced-stage CHL (AsHL). We evaluated the prognostic impact of a series of immunohistochemical markers on tissue micro-arrays assembled in Bologna from 138 of the patients enrolled in the above mentioned study.

In particular, eight parameters were assessed according to SanchezAguilera (Blood 2006): STAT-1, PCNA, SAP, TOP2A both in neoplastic (HRSC) and microenvironment cells (MC), RRM2, CDC2, MAD2 in HRSC, ALDH1A1 in MC. Moreover, CD 20, EBER, Bcl-2 and p53 were evaluated in HRSC. All patients had been treated with standard $\mathrm{ABVD} \pm \mathrm{Rx}$ therapy. Interim-PET after $2 \mathrm{ABVD}$ courses was evaluated according to the criteria of the JCO study. The mean age was 33.3 years (14-79), the stage III-IVB in 98 and IIB in 40, and the mean follow-up 38.1 months (7.6-71.9). Histopathology review showed: NS-I 75, NS-II 22, MC 20, DL 3, and CHL-NOS 18 cases. Interim-PET was positive in 30 patients, while treatment failure was recorded in 32. In unvaried analysis the factors related to treatment outcome were Bcl-2 on HRSC (cut off value 50\%), STAT-1/ SAP on MC, and PET (log-rank 6.9, 7.9 and 93.9 respectively). The combined expression of STAT-1 and SAP was scored in three levels depending on the architectural pattern: score 0 (expression of both with a diffuse/rosetting pattern); score 1 (discordant: combination of diffuse/rosetting and scattered patterns); score 2: (both markers with a scattered pattern); the 3-y PFS were $87.4 \%, 69.9 \%$ and $61.9 \%$ respectively. In multivariate analysis PET, Bcl-2 and STAT-1/SAP remained significant (HR: 24.8, 4.6, 7.5 and 5.6, respectively; $\mathrm{p}<0.01$ ). The proposed model is able to predict treatment response in AsCHL, but with a lower efficacy than PET. However, unlike PET, it can be applied upfront therapy.
LS 16

\section{NODULAR LYMPHOCYTE PREDOMINANT HODGKIN LYMPHOMA (NLPHL) WITH SPECIAL REFERENCE TO VARIANT PATTERNS AND SUB-TYPES PROGRESSING TO AGGRESSIVE DIFFUSE LYMPHOMAS}

\author{
(*) Martine Vornanen (1), Martin-Leo Hansmann (2) \\ Centre for Laboratory Medicine, Tampere University Hospital, \\ Tampere, Finland \\ Department of Pathology, Universitätsklinikum, Frankfurt/Main, \\ Germany
}

Background: NLPHL is now recognized as an indolent B-cell neoplasm belonging to the Hodgkin group. It is thought to originate from germinal center-derived B cells. It is a rare subtype of Hodgkin lymphoma, presenting usually as a localized disease that showed over the years a strong tendency to recurrence and, in a small percentage of cases, to transformation into a more aggressive type of lymphoma, usually DLBCL or TCRBCL-like type. Due to the rarity of this entity the histological and immunohistochemical findings encountered during the different phases of progression and transformation are poorly known and so is their outcome.

From the files of our institute we retrieved 41 patients with a diagnosis of LPHL or NLPHL. 48 blocks were collected from 40 patients and histologically reclassified using a large panel of immunohistochemical antibodies. 3 cases were excluded due to their reclassification as $\mathrm{cHL}$.

Results: In the NLPHL group we identified 4 different subgroups on the basis of growth pattern, cell component and immunohistochemical findings.

1- 11 cases were classified as classical NLPHL with a B-cell rich background identical to the classical nodular or serpiginous pattern. By IHC L\&H cells did not expressed CD19 and presented inconstant expression of EMA.

2- In 13 cases classified as intermediate group, we could notice a preservation of nodularity, but an increase in L\&H cells in follicles. In some central part of the nodules vanishing of background B-cells was often noticed with their replacement by small T-cells. In those T-cell rich areas slight positivity of L\&H cells for CD19 was noticed. Also EMA was inconstantly expressed with stronger positivity detected only in 2 cases.

3- 4 cases were classified as nodular with an excess of blasts, often in a tumoral or neoplastic pattern, in which the pleomorphic centroblastic -looking blasts were forming neoplastic groups in the nodules. They showed an immunophenotypic profile more in favour of germinal differentiation with expression of CD10 in 2 cases. EMA was also strongly expressed by the blastic population.

4- The last 9 cases presented with a predominantly diffuse growth pattern in a background of T-cells consistent with TCRLCB-like paragranuloma. In 8 cases $\mathrm{L} \& \mathrm{H}$ cells showed strong expression of CD19 as well as EMA.

The subtypes of NLPHL involved in progression or transformation are difficult to classify in any described lymphoma group and therefore are sometimes difficult to recognize as NLPHL. Specially the two last 
groups seemed to represent an aggressive form of the disease and some were associated with recurrent or clinically aggressively behaving disease.

\section{LS 17}

\section{ROLE OF CHROMATIN REMODELLING MEDIATED BY THE HIV-1 TAT PROTEIN IN THE GENESIS OF HIV-ASSOCIATED MALIGNANCIES}

Giulia De Falco*, Giovanna Cerino, Anna Luzzi, Alessandra Pasquarella, Federica Morettini, Francesco Imperatore, Piero Tosi and Lorenzo Leoncini

Dpt. Human Pathology and Oncology, University of Siena, Italy

The incidence of non-Hodgkin's lymphoma (NHL) is greatly increased in HIV-infected individuals. Although the molecular mechanism underlying HIV-mediated transformation is not clearly understood, the Tat protein of HIV is a likely candidate to contribute to tumour pathogenesis in HIV-infected patients. Extensive evidence indicates that Tat is a cofactor in the development of AIDS-related neoplasms and the protein has also been found to have an oncogenic role in vitro and in vivo. The molecular mechanism underlying Tat's pleotropic activity may include the generation of functional heterodimers of Tat with cell cycle proteins, but this may not be sufficient for neoplastic transformation in vivo.

Another mechanism of Tat-mediated transformation is by hyperactivation of transcription due to chromatin remodelling complexes. Recent findings indicate a complex interplay between viral proteins and host transcription regulatory machineries. The chromatin structure presents a significant barrier to transcription. These modifications and alterations of chromatin structure increase DNA accessibility to transcription factors and activators, thus promoting transcription initiation and efficient elongation. Many reports in the last several years have linked Tat transactivation to chromatin remodelling in vitro and in vivo.

We aimed to investigate whether Tat-mediated chromatin remodelling may have a role in HIV-associated transformation. Our results show that Tat is able to modulate the expression of some chromatin remodellers. In particular, cell lines transfected with Tat show an upregulation of $\mathrm{p} 300$, which is responsible for histone acetylation, and a dramatic down-regulation of DNMT1, which regulates DNA hypermethylation. Such a condition may result in a constitutively open conformation of the chromatin, which may eventually contribute to malignant transformation. We investigated the molecular mechanism underlying this event and demonstrated that it may rely on the Tatmediated modulation of specific microRNAs, which have these genes as targets. Interestingly, these findings were further confirmed in primary tumors of AIDS-related lymphomas.

\section{LS 18}

\section{HYPERMUTATION OF IMMUNOGLOBULIN GENES AND A LOW GENOMIC INSTABILITY DEFINE A NEW SUBTYPE OF MANTLE CELL LYMPHOMA WITH VERY INDOLENT OUTCOME}

*Fernàndez V, Salamero O, Jares P, Espinet B, Solé F, Agostinelli C, Koerbel L, Rozman M, Beà S, Aymerich M, Colomer D, Villamor N, Lloveras N, Fernández C, Bosch F, Swerdlow S, Pileri S, Montserrat E, Rosenwald A, Serrano S, Campo E

Hospital Clinic and Hospital del Mar, Barcelona, Spain; University of Würzburg, Germany; University of Bologna, Italy, University of Pittsburg, PA, USA

Purpose: MCL has usually an aggressive clinical behavior and poor response to therapy. However, a small group of patients may have a long survival and may not require chemotherapy treatment for a long period of time. The criteria to identify these patients are not defined. The goal of this study was to identify clinical and molecular features of MCL patients with a very indolent clinical outcome.

Patients and methods: We studied 12 patients with a very indolent MCL (iMCL) that were not treated (7) or had only splenectomy (5) and were followed for more than 2 years (median 60 months, 25-108) without the need of additional therapy. These cases were compared with 15 conventional MCL (cMCL) diagnosed during the same period of time. All cases had the $\mathrm{t}(11 ; 14)(\mathrm{q} 13 ; \mathrm{q} 32)$ and cyclin $\mathrm{D} 1$ overexpression. The mutational status of the immunoglobulin genes $\left(\mathrm{IgV}_{\mathrm{H}}\right)$ was examined in $8 \mathrm{iMCL}$ and $15 \mathrm{cMCL}$. The genomic profile of $7 \mathrm{iMCL}$ and $15 \mathrm{cMCL}$ was examined with $100 \mathrm{~K}$ SNP arrays using DNA extracted from purified ( $>98 \%$ ) peripheral blood tumor cells.

Results: iMCL patients had leukemic presentation (11/12), splenomegaly (6/12) and no (10/12) or very small isolated (2/12) detectable lymph nodes whereas these features in cMCL were present in 13/15, $6 / 15$ respectively, and significant lymphadenopaty in all patients, with only one patient with localized disease (stage IIA). All iMCL had hypermutated $(>5 \%) \operatorname{IgV}_{\mathrm{H}}$ genes. Seven $(45 \%)$ cMCL had $\operatorname{IgV}_{\mathrm{H}}$ mutations $(>2 \%)$ but only three $(20 \%)$ had a high rate of mutations $(>5 \%)$. The iMCL had no (5/7) or only one (2/7) genomic imbalances (3p26-p25 loss, an uncommon finding in cMCL; 17pter-p12 loss not associated with p53 mutations). On the contrary, 13/15 (87\%) cMCL had $\geq 2$ chromosomal alterations. $3 / 7$ iMCL and 7/15 cMCL had partial Uniparental Disomies (pUPD) that were also present in the germ-line of the patients except two recurrent somatic pUPDs (17pterp12, 20q) identified only in cMCL.

Conclusion: Non-Nodal MCL with high rate of $\mathrm{IgV}_{\mathrm{H}}$ gene hypermutations and lack of genomic complexity identify a subset of MCL patients with an indolent clinical course that may not need chemotherapy for a long period of time. 
LS 19

\section{microRNAs EXPRESSION AND CHROMOSOMAL ALTERATIONS IN MANTLE CELL LYMPHOMAS}

Alba Navarro ${ }^{1}$, Silvia Beà ${ }^{1}$, Verónica Fernández ${ }^{1}$, Miriam Prieto ${ }^{1}$, Itziar Salaverria ${ }^{1}$, Anna Mozos ${ }^{1}$, Armando López-Guillermo ${ }^{2}$, Neus Villamor ${ }^{2}$, Dolors Colomer ${ }^{2}$, Xavier Puig ${ }^{3}$, Andreas Rosenwald ${ }^{4}$, Elías Campo ${ }^{1},{ }^{*}$ Luis Hernández ${ }^{1}$

Departments of ${ }^{1}$ Pathology and ${ }^{2}$ Hematology, Hospital Clinic, University of Barcelona, Barcelona, Spain. ${ }^{3}$ Department of Statistics, Technical University of Catalonia, Barcelona, Spain. ${ }^{4}$ Institute of Pathology, University of Würzburg, Germany.

Background: Mantle cell lymphomas (MCL) usually showed a high number of recurrent chromosomal aberrations, potentially affecting regulatory RNAs known as microRNAs (miRs) and eventually leading to their abnormal expression with potentially relevant pathogenetic implications.

Purpose of the study: To determine the expression profile of a panel of $85 \mathrm{miR}$ encoded by chromosomal region commonly altered in MCL and its potential relationship with clinical and biological characteristics of the tumors. miR expression was assessed by looped-qPCR in CD5+ and CD5- B-cells purifed form reactive tonsils, a panel of $8 \mathrm{MCL}$ cell lines, 16 samples of purified tumor cells from peripheral blood of MCL at diagnosis, and 12 samples of primary nodal tumors tissue. Genomic alterations were examined by SNP-arrays. Two paired samples of purified tumor cells from peripheral blood and lymph node were also examined.

Results: The miR examined showed a different expression profile in MCL and both CD5 positive and negative B-cells. The expression levels of most miR were not related to the copy number changes of the respective chromosomal loci or the $t(11,14)$ translocation. However, the expression of the oncogenic miR17-92 cluster in $13 \mathrm{q} 31$ was significantly related to the gain/amplification of this chromosomal region. High expression of these miRs was frequently associated with high $M Y C$ mRNA levels in patients with a more aggressive behavior. Finally, we have found different $m i R$ expression profiles according to microenvironment or Ig mutational status of MCL tumor cells

Conclusion: miR17-92 cluster seems the target of the 13q31 gain/ amplifications in MCL. The miR expression profile in MCL differs from both $\mathrm{CD} 5+$ and - non-neoplastic B cells and it is not related to the genomic changes of the respective chromosomal loci. The differential expression in purified cells from peripheral blood and tissue samples suggest a potential influence of the microenvirontment in the modulation of miR in MCL.
LS 20

\section{PREFERENTIAL IMMUNOGLOBULIN VARIABLE HEAVY CHAIN USAGE AND STEREOTYPED PATTERNS OF SOMATIC HYPERMUTATION IN PATIENTS WITH CHRONIC LYMPHOCYTIC LEUKEMIA AND MANTLE CELL LYMPHOMA}

Paul Rombout ${ }^{1}$,Margit Schraders ${ }^{1}$, Anne Jansen ${ }^{1}$,Martijn de Prenter $^{1}$, Reinier Raymakers ${ }^{2}, \mathrm{MD}, \mathrm{PhD}$, Konnie Hebeda ${ }^{1} \mathrm{MD}$, $\mathrm{PhD}$, Johan van Krieken ${ }^{1} \mathrm{MD}, \mathrm{PhD}$ and Patricia JTA Groenen ${ }^{1} \mathrm{PhD}^{*}$

Dept. of Pathology ${ }^{1}$, dept of Hematology ${ }^{2}$, Radboud University Nijmegen Medical Centre, Nijmegen, The Netherlands.

Background: In chronic lymphocytic leukemia (CLL), biased usage of the immunoglobulin variable heavy (IGHV) chain and stereotyped patterns of somatic hypermutation (SH) occur, particularly in IGHV321 and IGHV4-34 subsets. Both features provide evidence for antigen selection in CLL pathogenesis. In mantle cell lymphoma (MCL), there is preferential usage of $I G H \mathrm{~V} 3-21$ and $I G H \mathrm{~V} 4-34$, however there is no evidence for a prognostic role of IGH $\mathrm{SH}$, nor for antigen selection.

Aim of study: To investigate the possibility of antigen-driven pathogenesis in Dutch CLL and MCL patients by determination of the CDR3 region, the VH-family and light chain usage.

Results: We determined the IGH VDJ rearrangement from blood ( $\mathrm{n}=$ $61)$ or lymph nodes $(n=41)$ of CLL patients, from lymph nodes $(n=$ 27) of MCL patients, and the light chain rearrangements of IGHV 169 and 3-21 -cases.

In CLL there is specific usage of IGHV 1-69 (17,6\%), IGHV 3-21 $(5,8 \%)$ and $I G H \mathrm{~V} 4-34(5,8 \%)$. In the $I G H \mathrm{~V} 1-69$ subgroup, there is preferential usage of $\mathrm{D}$ and $\mathrm{J}$ gene segments. Half of the $I G H \mathrm{~V}$ 1-69 subgroup display homology in the CDR3 region. There is however no preferential usage nor homology in the CDR3 region of the light chain rearrangements of $I G H \mathrm{~V} 1-69$ or $I G H \mathrm{~V}$ 3-21 cases.

Usage of $I G H \mathrm{~V}$ 1-69 does not occur in MCL. The IGHV 3-21 MCL cases (28\%) display homology in the CDR3 region but the Lambda $\mathrm{V}$ gene usage is not uniform. Stereotyped patterns of SH are found in 2/6 $I G H \mathrm{~V}$ 3-21 and 1/6 IGHV 4-34 CLL cases and interestingly also in 2/5 IGHV 4-34 MCL cases.

In conclusion: Biased V-segment usage and stereotyped patterns of $\mathrm{SH}$ do occur in Dutch CLL and MCL patients. The findings in MCL address the question whether there is involvement of antigen selection in MCL pathogenesis, as is suggested in CLL pathogenesis.

\section{LS 21}

\section{PITTFALLS IN TCR CLONALITY TESTING}

Patricia JTA Groenen, $\mathrm{PhD}^{1}$, Anton W Langerak, $\mathrm{PhD}^{2}$, Jacques JM van Dongen, $\mathrm{MD}, \mathrm{PhD}^{2}$ and Johan HJM van Krieken, $\mathrm{MD}, \mathrm{PhD}^{1}$,

${ }^{1}$ Dept. of Pathology, Radboud University Nijmegen Medical Centre, Nijmegen, Netherlands ${ }^{2}$ Dept. of Immunology, Erasmus MC, University Medical Center, Rotterdam, Netherlands 
Background: T-cell clonality testing in lymphoproliferations has technically become relatively easy to perform in routine laboratories using standardized multiplex PCR protocols for T-cell receptor (TCR) gene analysis. Expertise with clonality diagnostics and knowledge about the biology of the TCR recombination are essential for correct interpretation of the TCR clonality data.

Description of results: To ascertain high quality and reproducible clonality assessment and to avoid misinterpretation of the data, DNAquality control, controlled DNA-input and tissue reproducibility, are essential quality parameters to evaluate in the routine diagnostic setting. Measuring peak intensities or area under the peak is not the way to interpret clonality results. A quantitative approach will lead to missed clonal processes and also to wrongly called clonal cases. In this respect, it is better to use the histopathological information on the presence of lymphocytes and suspicious malignant lymphocytes, and to use the knowledge of the PCR target and the multiple target approach, to reach a correct interpretation of the clonality results. Clonality interpretation should also be performed the context of immunobiology. A pitfall concerns detection of oligoclonality of even (low level) monoclonality of T-lymphoctyes under certain immunobiologic conditions.

In conclusion: Several technical and immunobiological pitfalls should be taken into account to avoid misinterpretation of data. For appropriate interpretation of TCR clonality, it is necessary to integrate the molecular data with that from the pathology, and preferably also flowcytometric immunophenotyping. Such an interactive, multidisciplinary diagnostic model guarantees integration of all available data to reach the most reliable diagnosis.

\section{$\underline{\text { LS } 22}$}

\section{IDENTIFICATION OF THE CORE PHOSPHOPROTEOME OF MANTLE CELL LYMPHOMA CELL LINES}

Daniela Cecconi ${ }^{1}$, Elena Bianchi ${ }^{1}$, Alice Parisi $^{2}$, Stefano Barbi ${ }^{2}$, Alberto Milli ${ }^{1}$, Sara Rinalducci ${ }^{3}$, A. Rosenwald ${ }^{4}$, Elena Hartmann ${ }^{4}$, Lello Zolla ${ }^{3}$, Marco Chilosi ${ }^{2}$, and Alberto Zamò ${ }^{2 *}$

1 University of Verona, Dipartimento Scientifico e Tecnologico, Laboratorio di Proteomica, Verona, Italy

2 University of Verona, Dipartimento di Patologia, Sezione di Anatomia Patologica, Verona, Italy

${ }^{3}$ University of Tuscia, Dipartimento di Scienze Ambientali, Viterbo, Italy

${ }^{4}$ University of Wuerzburg, Department of Pathology, Wuerzburg, Germany

Background: Mantle cell lymphoma (MCL) is an incurable hematologic malignancy whose pathogenesis is only partly understood. Few studies have attempted the proteomic approach to decipher its pathogenesis.

Purpose of the study: The aim of our study was the definition of a "core phosphoproteome", shared among all MCL cell lines, that should be representative of real MCL samples. These data might improve the understanding of the signal transduction pathways involved in MCL tumorigenesis and suggest new potential therapeutic targets. We have sorted the phosphorylated proteins of several MCL cell lines by means of immobilized metal affinity chromatography and separated them by 2D-PAGE, followed by RP-HPLC coupled with MS/
MS identification. These findings were then correlated with information regarding copy number gains obtained by snp-chip analysis.

Results: Several of the identified proteins could be linked to a specific signal transduction pathway. Some of these (like NF-kB and PI3KmTOR) were recently recognized as important players in MCL pathogenesis, but a our data provide an independent proof, and suggest a number of involved molecules not previously demonstrated. Other pathways, like mitochondrial signalling, constitute a rather new finding in MCL pathobiology. A second-level analysis identified MAPK1, CK2, CK1, PKCzeta and PKCepsilon as candidate upstream actors. The correlation with snp-chip analysis found a good correlation with some interesting molecules, including ST13 and FKBP4.

Conclusion: Our study provides new insights in MCL pathogenesis and may suggest new potential therapeutic targets.

\section{LS 23}

\section{CLINICOPATHOLOGICAL HETEROGENEITY IN PLASMABLASTIC LYMPHOMA}

*Tomomi Billings, M.D., Jane Tongson-Ignacio, M.D., Xiaohui Zhao M.D., Ph.D. Department of Pathology and Laboratory Medicine, University of California Irvine School of Medicine, Irvine, California, U.S.A

Background: Plasmablastic lymphoma (PBL) is classified as a rare variant of diffuse large B-cell lymphoma (DLBCL) under the WHO classification. Although PBL shares similar morphologic features with DLBCL, it appears to differ in other clinicopathological aspects.

Purpose: To demonstrate the diverse clinical and histopathological features of PBL.

Results: Eight cases of PBL were evaluated. Of eight cases (2 female:6 male, 30-72 years of age), five were HIV-positive, one HIVnegative, and two with an unknown HIV status. One had an isolated nodal location and seven had an extranodal location (3-nasopharynx, 2-colorectum, 1-anorectum and 1-ovary) with an involvement of ascitic fluid and/or bone marrow in some cases. All tumors showed diffuse large round cell/blastic morphology, three of which showed additional plasmacytic cytomorphology. The tumor cells were negative for CD20 (8/8) and PAX5 (4/4) by immunohistochemistry (IHC) with an immunophenotype indicative of plasma cell differentiation (MUM1/CD38/CD138-positive) and light chain restriction by IHC and/or in-situ hybridization (ISH) (4 kappa and 3 lambda). They were variably positive for CD45 (4/8), CD79a (2/5), CD30 (1/7) and EMA (4/7). EBER was positive in six (5 HIV-positive and 1 HIVnegative) out of eight cases, while HHV8 (IHC) was positive in only one case (HIV-positive) which also showed EBER positivity. On follow-up, three patients (2 HIV-positive and 1 with an unknown status) died of multiorgan involvement within 6 months after the initial diagnosis, two HIV-positive patients were alive at 6- and 15-months, and the remaining three patients were lost to follow-up. Conclusion: PBLs are poorly differentiated plasmacytic neoplasms with diffuse large cell/blastic morphology, plasmacytic immunophenotype, and lymphoma-like behavior. However, PBLs are heterogeneous entities exhibiting variable characteristics in tumor location, association with EBV and HHV8, and clinical course. They commonly, but not exclusively, occur in HIV-positive patients. Recognition of this rare entity is essential for proper management. 


\section{LS 24}

\section{IMMUNOPHENOTYPIC VARIATIONS OF BURKITT LYMPHOMA}

*Katalin Kelemen, Rita Braziel, Ken M. Gatter, James Z. Huang, Guang Fan

Department of Pathology, Oregon Health Science University, Portland, OR, USA

Burkitt lymphoma (BL) exhibits a characteristic germinal center B-cell immunophenotype that is positive for pan-B-cell markers, CD10 and expresses clonal surface immunoglobulin (sIg) light chains. The frequency of atypical immunophenotype in Burkitt lymphoma detected by flow ctytometry (FC) has not been established. The aim of this study is to analyze the frequency and spectrum of aberrant immunophenotypes in Burkitt lymphoma.

33 cases of BL were studied. The diagnosis of BL was established based on morphology, immunophenotype and cytogenetic findings detected by FISH and/or conventional karyotyping. $51 \mathrm{FC}$ analyses were performed on the $33 \mathrm{BLs}$ and all results were reviewed.

Of 33 BLs, 4 exhibited aberrant FC immunophenotype (12.1\%). All four cases with aberrant immunophenotypes had cytogenetic confirmation of the diagnosis. In two of the four patients, lack of sIg light chain expression was the only observed aberrancy; both of these patients expressed clonal cytoplasmic Ig light chains. Two cases exhibited complex phenotypic aberrancies. One of these lacked both surface and cytoplasmic Ig light chains and was negative for CD19 and $\mathrm{CD} 22$, expressed CD10, $\operatorname{dim} \mathrm{CD} 20, \operatorname{dim} \mathrm{CD} 45$, and CD79a in addition to CD4, CD38 and CD71. Another case expressed clonal sIg light chains and expressed CD79 but lacked CD10 and CD20. The phenotypic aberrancies were reproduced by immunhistochemistry in both cases. Aberrant immunophenotypes were observed in both pediatric and adult $\mathrm{BL}$ cases, and in one patient, the BL represented a post-transplant lymphoproliferative disorder.

Aberrant FC immunophenotypes are detected in $12 \%$ of BL cases, including markedly deviant phenotypes from the classic phenotype. Recognizing the variability of immunophenotype and correlation with morphologic and cytogenetic findings is essential for establishing an accurate diagnosis of BL.

\section{LS 25}

\section{UPREGULATION OF SERINE PROTEASE GENE TMPRSS 2 IN HODGKIN LYMPHOMA CELL LINES.}

Faisal Fadlelmola, Minglong Zhou and *Diponkar Banerjee.

British Columbia Cancer Agency, Centre for Translational and Applied Genomics

Department of Pathology and Laboratory Medicine, Vancouver, BC, V5Z 4E6, Canada.

Background: The human genome contains 612 genes encoding proteases or protease-like molecules, 389 non-protease homologs, 115 protease inhibitor genes, and 180 non-peptidase homologs, now termed the "degradome", comprising over $2 \%$ of the human genome. Human proteases belong to five different catalytic classes (Aspartic, cysteine, and threonine proteases, metalloproteases and serine proteases). Metalloproteases and serine proteases are the most common (187 and 175 genes respectively). There are over 90 protease genes that through evolution have lost essential residues necessary for proteolytic activity and are classified as non- protease homologs. These inactive proteases, however, can regulate the activation of other proteases or compete with their substrates or inhibitors.

Purpose of the study: To examine whether or not protease genes are altered in Hodgkin Lymphoma. Five HL cell lines were surveyed for the expression of protease genes in comparison to benign germinal centre B cells. For genes shown to be altered, confirmatory quantitative PCR (q-PCR), Western blots and immunofluorescence assays were performed.

Results: TMPRSS2 was upregulated in $2 \mathrm{HL}$ cell lines. Western blots probed with anti-TMPRRS2 protein showed the presence of 2 bands in HL cell lines, with molecular sizes of $32 \mathrm{kDa}$ and $50 \mathrm{kDa}$, the $32 \mathrm{kDa}$ band representing the extracellular domain of TMPRSS2 protein. Immunofluorescence labelling with anti-TMPRSS2 protein showed strong membrane staining. Q-PCR showed 37,000 fold relative expression of TMPRSS2 in L428 cells in comparison to diffuse large B cell lymphoma derived B cells, house-keeping genes, or control universal human reference RNA.

Conclusions: TMPRSS2, a type II transmembrane serine protease, is upregulated in some but not all HL cell lines tested and the protein is expressed on the cell membrane. The functional significance of these findings and the presence of TMPRRS2 protein in clinical samples of $\mathrm{HL}$ are being investigated.

\section{LS 26}

\section{SOX11 TRANSCRIPTION FACTOR IS CONSISTENTLY EXPRESSED IN PRECURSOR CELL LYMPHOBLASTIC NEOPLASIA}

*Michael Dictor, Department of Pathology, Lund University Hospital Sara Ek and Carl Borrebaeck, Department of Immunotechnology, Lund University, Lund, Sweden

Sox transcriptional proteins regulate fundamental developmental processes such as embryonic stem cell differentiation, neurogenesis and chondrogenesis. Recently, we confirmed the nearly consistent nuclear expression of Sox11 in mantle cell lymphoma (MCL) but not in its common simulators. We have now studied additional categories of lymphoma to gain a possible clue as to the biology of Sox11 in lymphoid cells and determine its usefulness in diagnosis.

Lymphoma/leukemias of type marginal zone ( 2 splenic, 1 nodal and two extranodal MZL), Burkitt (8 BL), T and B-lymphoblastic (10 TLL and $12 \mathrm{~B}-\mathrm{ALL} / \mathrm{LL})$, hairy cell (12 HCL), mature T-cell (3 Alk-1 ${ }^{+}$ ALCL, 1 AIL and 6 peripheral TCL-US), NK/T-cell (4) and Hodgkin (5 classic and 1 HL-LP) were included, in addition to 9 cases of myeloma/plasmacytoma. HIER-treated sections were immunostained with a rabbit polyclonal anti-human Sox11 antibody followed by Envision (Dako)/ DAB.

Sox11 nuclear signal was present consistently in precursor cell T-LL and B-ALL/LL (each included $2 \mathrm{TdT}^{-}$cases), in 4 of $8 \mathrm{BL}$ and 2 of $12 \mathrm{HCL}$ but absent in myeloma, HL and other mature B and T-cell neoplasms. As in MCL, most cases of T-LL expressed Sox11 only in the nucleus, whereas B-ALL/LL and BL stained in both nucleus and cytoplasm. Myeloma and MZL showed prominent granular staining confined to the 
cytoplasm, similar to that noted previously in chronic lymphatic leukemia/small lymphocytic lymphoma and follicular lymphoma.

The occurrence of weak cytoplasmic signal in benign germinal center cells, frequent strong cytoplasmic signal in B-cell neoplasms and both nuclear and cytoplasmic Sox11 protein in B-ALL/LL, BL and some MCL suggests that Sox 11 frequently accumulates in the perinuclear cytoplasm in benign and malignant B-cells and is shuttled into the nucleus in a narrow subset of lymphoid neoplasia.

Nuclear Sox 11 is a highly sensitive marker for precursor cell $\mathrm{T}$ and B- ALL/LL, in addition to MCL.

\section{LS 27}

\section{THE LYN KINASE AND THE Cbp/PAG ADAPTOR SELECTIVELY ASSOCIATE IN THE PLASMA MEMBRANE RAFTS OF HUMAN NON-HODGKIN LYMPHOMAS AND PROMOTE SURVIVAL.}

\author{
B. Borisch*, S. Tauzin, H. Ding and D.C. Hoessli
}

Department of Pathology and Immunology, Centre Medical Universitaire, Geneva, Switzerland.

Human cell lines from follicular, mantle-cell, diffuse large B-cell and Burkitt-derived lymphomas associate the non-receptor Lyn kinase and the $\mathrm{Cbp} / \mathrm{PAG}$ adaptor, a widely distributed lymphoid signaling molecule, in form of a signalosome in sphingolipid-rich plasma membrane rafts. This Lyn/Cbp/PAG signalosome also comprises the phosphatidylinositol-3' kinase and phosphorylated STAT3 that both connect the signalosome with pro-survival pathways. Upon inhibition of Lyn with specific Gleevec-related small molecules, all cell lines except Burkitt-derived ones undergo apoptosis, suggesting that acute blockade of prosurvival pathways reveals the activity of intrinsic apoptotic pathways in those cells (oncogenic shock). Inhibition of Lyn decreases Cbp/PAG tyrosine phosphorylation and partially dissociates the signalosome from rafts. Decreasing expression of Lyn or $\mathrm{Cbp} /$ PAG with siRNAs induces similar apoptotic responses, suggesting that the Lyn-Cbp/PAG signalosome constitutes an oncogenic component in B-NHLs. The Lyn and Cbp/PAG signaling proteins are also expressed in Hodgkin and ALK+ lymphoma lines, but do not assemble in oncogenic signalosomes, and the cells do not undergo apoptosis upon Lyn inhibition.

The $\mathrm{Cbp} / \mathrm{PAG}$ adaptor is considered to maintain Lyn in a constitutively activated state which cannot be altered by the cytoplasmic Csk kinase, the negative regulator of Lyn. This modality of Lyn regulation appears to be unique to B-NHLs and stands in contrast to what has been described in human T lymphocytes and mast cells, where Csk binds to the transmembrane $\mathrm{Cbp} / \mathrm{PAG}$ and negatively regulates Lyn. The Csk kinase shows negligible modular interaction with the optimally tyrosine phosphorylated Cbp/PAG of B-NHLs, and is not part of the signalosome.

Our results disclosed a unique kind of proximal, constitutive signaling in B-NHLs and suggest that therapeutic approaches focusing on the plasma membrane are likely to alter the Lyn-Cbp/PAG signalosome and facilitate destruction of lymphoma cells "addicted" to the Lyn kinase.

\section{LS 28}

\section{EXPRESSION OF MUM-1, OCT-2 AND BCL-6 IN SYSTEMIC ANAPLASTIC LARGE CELL LYMPHOMAS}

\author{
E. Arzu Saglam*, Aysegul Uner
}

Hacettepe University Faculty of Medicine, Department of Pathology, Ankara, Turkey

Recently, there has been an expansion in the array of transcription factors available in diagnostic hematopathology. Several transcription factors predominantly used for B cell lineage identification and classification are also expressed in a small percentage of $\mathrm{T}$ cells within germinal centers (GCs) and interfollicular areas. The aim of this study was to evaluate the immunohistochemical expression of Mum-1, Oct2 and Bcl-6 in systemic anaplastic large cell lymphomas (ALCL). 30 cases of ALCL were retrieved from our archives and tissue microarray constructed. Immunohistochemistry was carried out using an avidinbiotin peroxidase complex method.

Twenty five of the cases were of the T cell phenotype, 5 of null cell type. There were 15 ALK positive and 15 ALK negative cases. CD30 positivity was present in all cases. A predominance of nuclear staining was observed for all transcription factors. Mum-1 was positive in all but one case (96.7\%). Half of the cases displayed Oct-2 expression (15/30 cases). Diffuse nuclear staining with moderate to strong intensity was observed in all positive cases with Mum-1 and Oct-2. A considerable number of cases also had Bcl-6 expression (9/30). Bcl6 however mainly demonstrated focal and weak nuclear staining. Our findings emphasize that these markers are not restricted to B cell lineage and that extensive expression can be observed in anaplastic large cell lymphomas of $\mathrm{T} /$ null cell phenotype. The significance of this observation requires further evaluation.

\section{LS 29}

\section{HIGLY AGGRESSIVE ALK-1 (+) ANAPLASTIC LARGE CELL LYMPHOMA WITH MULTI-ORGAN INVOLVEMENT**}

\author{
*BAHAR KILICARSLAN AKKAYA(1), GULSUN TEZCAN(2), \\ GUL ALKAN(1), VOLKAN HAZAR(2)
}

AKDENIZ UNIVERSITY SCHOOL OF MEDICINE PATHOLOGY (1) AND PEDIATRIC HEMATOLOGY-ONCOLOGY(2) DEPARTMENTS, ANTALYA-TURKEY

Anaplastic large cell lymphoma (ALCL) is a rare tumor comprising 10-15 of childhood lymphomas. Primary and secondary involvement of nervous system is extremely rare in ALCL.

An 11-year-old child presented with fever and backache lasting for more than 20 days. In physical examination, the only pathological findings were microlymphadenopathies in cervical region and palpable liver below the costal margin. Chest X-ray showed bilateral pleural effusion. Because of unresponsiveness to empirical antibiotic treatment, she was planned to be evaluated in regard of malignancy. Bone marrow aspiration was dry tap. Thorax CT showed anterior mediastinal lymphadenopathies and also involvement of right lung. Abdominal CT demonstrated milimetric hypodens lesions in liver. Spinal MRI showed posterior epidural mass in T8, T9, T10 regions. Also diffuse skeletal infiltrations in cervical, thoracal and lumbosacral 
regions consistent with infiltrative disease. Cranial MRI revealed diffuse dural enhancement in favor of malignancy. Biopsy showed proliferation of single and poorly cohesive groups of cells with large pleomorphic nuclei, containing prominent nucleoli, and moderate amount of cytoplasm on rich histiocytic backround on epidural mass. In differantial diagnosis histiocytosis and lymphoma were considered. Immunohistochemical staining revealed CD30, CD43, EMA and ALK-1 positivity that is typical of ALCL, a rare form of T-cell lymphoma. She was accepted as stage IV due to CNS and bone marrow involvement. Chemotherapy according to NHL-BFM chemotherapy protocol was started. After two blocks of therapy, pulmonary and hepatic lesions disappeared. Although clinical improvement was obvious, cranial and spinal lesions existed. The chemotherapy has been still continued and after the end of 6th therapy element, she is planned to be evaluated with PET scan.

Conclusion: Involvement nervous system of ALCL is very rare. Early recognition of ALCL by biopsy is important to order therapeutic approach and to prevent deteriorating clinical course.

**We would like to thank with a Group of Consultation for Lymphoma in Ankara, for providing us with support of diagnosis.

\section{$\underline{\text { LS } 30}$}

\section{METHYLATION OF $P 16^{I N K 4 A}$ AND MAD2 IN GASTRIC MARGINAL ZONE B-CELL LYMPHOMAS.}

Sanghui Park*, Kyoung-Mee Kim, Young-Hyeh Ko

Department of Pathology, Gachon University of Medicine and Science, Gil Medical Center, Incheon, and Samsung Medical Center, Sungkyunkwan University School of Medicine, Seoul, Korea

Background: The products of cyclin dependent kinase inhibitor $p 16^{\mathrm{INK} 4 \mathrm{~A}}$ and mitotic checkpoint gene $M a d 2$ are key regulator proteins at the G1 restriction point and mitotic checkpoint of the cell cycle.

Materials and Methods: To investigates the role of promoter methylation of $p 16^{\mathrm{INK} 4 \mathrm{~A}}$ and $M a d 2$ in gastric marginal zone B cell lymphoma (MZBCL), gastric biopsies from 40 patients were analyzed by methylation-specific PCR and the methylation status was compared with the results of BCL10 expression and $\mathrm{t}(11 ; 18)(\mathrm{q} 21 ; \mathrm{q} 21)$ translocation.

Results: $p 16^{\mathrm{INK} 4 \mathrm{~A}}$ was methylated in 29 of 38 MZBCLs $(76.3 \%)$. The lymphomas with $p 16^{\mathrm{INK} 4 \mathrm{~A}}$ methylation tended to respond well to Helicobacter pylori eradication $(\mathrm{p}=0.078)$ and were negative for $\mathrm{t}$ $(11 ; 18)(\mathrm{q} 21 ; \mathrm{q} 21)(p=0.040)$. Mad2 was methylated in 23 of 37 MZBCLs $(62.2 \%)$. The lymphomas with $M a d 2$ methylation more frequently expressed BCL10 $(p=0.052)$.

Conclusion: These methylation profiles suggest that $p 16^{\mathrm{INK} 4 \mathrm{~A}}$ and Mad2 may play a role in the pathogenesis of MZBCL in different way; Mad2 is H. pylori independent with a close association with BCL10 while $p 16^{\mathrm{INK} 4 \mathrm{~A}}$ is $H$. pylori-dependent with an inverse correlation with the $\mathrm{t}(11 ; 18)(\mathrm{q} 21 ; \mathrm{q} 21)$ translocation.

\section{LS 31}

\section{HIV- ASSOCIATED PRECURSOR T LYMPHOBLASTIC LEUKAEMIA/ LYMPHOBLASTIC LYMPHOMA: REPORT OF A CASE AND REVIEW OF LITERATURE.}

D Lorenzon, T Perin, V De Re, L Caggiari, M Michieli, M Spina, P Bulian, C. Colarossi§, V Gattei and *V Canzonieri.

From Servizio di Onco-Ematologia Clinico-Sperimentale, Anatomia Patologica, Farmacologia Sperimentale e Clinica, Oncologia Medica, I.R.C.C.S CRO, Aviano, Italy.

$\S I O M$ Viagrande, Catania

Background. T lymphoblastic leukaemia/lymphoblastic lymphoma (T-ALL/T-LBL) is a rare instance among HIV patients. A PubMed search yielded only 4 cases.

Aim. To report cytomorphologic, immunophenotypic, molecular and clinical features of a new case of T-ALL/T-LBL.

Results. A case of precursor T-ALL/T-LBL developed after nine years of HAART-treated HIV infection. At the onset the disease was diagnosed as T-LBL and was characterized by epatosplenomegaly and lymphocytosis. After 6 cycles of CHOP the patient went in complete remission for 9 months, followed by a relapse, treated with polychemotherapy. During subsequent remission an harvest of peripheral blood stem cell was attempted without success. After two months from consolidation a new relapse unresponsive to standard dose chemotherapy was treated with high dose ARA-C. The following complete remission lasted for about two months, during this period failed a second attempt at peripheral stem cell harvest. After relapsing the patient received palliative therapy for two months until death. At last relapse bone marrow biopsy showed a hypercellular marrow infiltrated by precursor $\mathrm{T}$ lymphoblasts with very condensed nuclear chromatin, no evident nucleoli and high number of mitotic figures. In smears the cells were medium size with high nuclear cytoplasmic ratio and cytoplasmic vacuoles. Lymphoblasts were positive for CD1a, cCD3, TdT, CD2, CD7, CD5, CD10, mainly CD8 with partial coexpression of CD4, with a proliferative fraction of $70 \%$. They were negative for EBV latent membrane protein (LMP) and human herpes virus 8 (HHV-8). In situ hybridization showed negativity for Epstein-Barr viral early RNA. Genescan profiles of TCR $\gamma$ PCR products showed two clonal peaks. Analysis of TCR sequences showed that one contained a stop codon.

Conclusions. A new T-ALL/T-LBL adds up to five the total of cases reported in the English literature and is the sole case with $\mathrm{CD} 8$ positivity.

\section{LS 32}

\section{RELATIONSHIP BETWEEN GALECTIN-10 EXPRESSION AND SEVERITY OF CELIAC DISEASE ABOLISHED IN THE PRESENCE OF GAMMADELTA-POSITIVE T CELL CLONAL EXPANSION.}

*V. Canzonieri, M.P. Simula,V. De Re, D. Marin, T. Perin, C. Colarossi§, R. Cannizzaro

Institute(s):Centro di Riferimento Oncologico, National Cancer Institute,Aviano, and §IOM, Viagrande, Catania, Italy.

Background: Celiac disease (CD) patients are prone to develop T-cell lymphoma throughout a progressive accumulation of aberrant, clonal 
$\mathrm{gd}+\mathrm{T}$-cells. It is supposed that high production of pro-inflammatory cytokines, and chronic antigenic stimulation, due to gluten ingestion, plays a key role in inducing inflammation, resistance to apoptosis and the emergence of these T-clones. Regulatory T-cells maintain immunological self-tolerance by active suppression of auto aggressive T-cells. Among them, Tr1 subset plays a role in the suppression of naïve and memory T-cells. The role of $\operatorname{Tr} 1$ in human diseases is not well understood, even because there are no specific markers able to identify these cells, however recently, Galectin-10 has been proposed as a marker for functional $\operatorname{Tr} 1$.

Purpose: To better understand pathogenetic mechanisms associated with $\mathrm{CD}$ and clonal T-cell proliferations we investigated galectin-10 expression from gut epithelium by 2D-DIGEapproaches. Patients were selected and grouped for histological damage degree and for $\mathrm{gd}+\mathrm{T}$ pattern defined by $\mathrm{g}$-TCR genescan analysis.

Results: Groups consisted of 7 individuals with Marsh-0 (4/7 oligoclonal), 3 with a Marsh-1 or -2 (3/3 polyclonal) and 5 with Marsh-3 (2/5 clonal $\mathrm{gd}+\mathrm{T})$. Control consisted of 4 individuals with excluded CD. We found, a parallel increase in galectin-10 levels and Marsh index from 1 to 3 in individuals with polyclonal $\mathrm{gd}+\mathrm{T}$ cells $(\mathrm{p}=0.0092)$, while reduced levels were evidenced from patients with clonal $\mathrm{gd}+\mathrm{T}$ cells / Marsh-3 $(\mathrm{p}=0.017)$.

Conclusion: We assume that galectin-10 up-expression may exert a protective role in limiting the mucosal damage. Such a role disappears in case of clonal $\mathrm{gd}+\mathrm{T}$ cells proliferation. Biopsy quantitation of eosinophils and CD25 + cell population producing galectin-10 are performing.Further studies are needed to establish if these clones are those more susceptible to malignant progression.

\section{$\underline{\text { LS } 33}$}

\section{CHARACTERIZATION OF ANTIBODIES DIRECT AGAINST THE IDIOTYPIC VK CHAIN OF HCV-RELATED TYPE-II MIXED CRYOGLOBULINEMIA AND B-CELL PROLIFERATIONS.}

V. Canzonieri, V. De Re, M.P. Simula, A. Pavan, L. Caggiari, T. Perin, S. Sansonno

Centro di Riferimento Oncologico, National Cancer Institute, Aviano, Italy.

Background: Autoimmune type-II cryoglobulinemia (MC) is sustained by clonal/oligoclonal B-cell populations so that the disease may be considered an "indolent B-cell lymphoma (NHL)" and may favor overt NHL development . HCV-antigen driven mechanism induces Bcell proliferations. Clonal B-cells demonstrate a restricted panel of variable genes to construct the B-cell receptor (BCR) and a homology between BCR functional regions and autoimmune rheumatoid factor (RF) activity. We underline the BCR unique repertoire with frequent rheumatoid factor activity also in other autoimmune disorders associated to NHL development as Sjogren's syndrome and rheumatoid arthritis. All together these BCRs are characterized by their high degree of idiotypic (Id) cross reactivity. Particularly the K chain V320/15 is frequently found in subject with positive HCV antibody. Id is a clonal marker expressed by B cells, thus is an ideal target for immunotherapy.
Purpose: The evidence of few Id presented in the NHL subgroup above reported constitute the rational for the development of antibodies and recombinant proteins that use shared Id among different NHL.

Results: Five monoclonal antibodies have been produced in our laboratory toward the VK3-20 region of a subject HCV+ with NHL. Epitopes recognized has been performed using epitope excision approach.

Conclusions: A fine determination of the antibodies activities toward specific amino acids, possibly common to different individuals, are in progress. Monoclonal antibodies reactivity will be produced in vitro in ELISA, western blot, cytofluorimetry and immunohistochemistry. Apoptosis and ERK1/2 phosphorylationpathways induced following BCR cross-linking of antiId murine IgG will be performed.

\section{LS 34}

\section{CLONAL CD27+ CD19+ B-CELL EXPANSION THROUGH INHIBITION OF FCgIIR IN HCV+ CRYOGLOBULINEMIC PATIENTS.}

V. Canzonieri, V. De Re, V. Racanelli, A. Pavan, T. Perin, S. Sansonno.

Centro di Riferimento Oncologico, National cancer Institute, Aviano, Italy, Università degli Studi di BARI, Bari, Italy.

Background: HCV infection may or may not be associated with extra-hepatic manifestations such as type-II mixed cryoglobulinemia (MC), a clonal B cell proliferative disorder. In persistent $\mathrm{HCV}$ infection without MC an increase in serum immunoglobulins (Ig) is commonly observed. We found this increase is polyclonal and is determined primarily by increased levels of both $\mathrm{HCV}$-specific and nonspecific IgG. Despite this hypergammaglobulinemia, memory CD27+ cells do not accumulate, depending on a heightened sensitivity of memory B cells to BCR-independent non cognate $\mathrm{T}$ cell help which speeds up their terminal differentiation into antibody secreting cells and make them more prone to activation induced cell death. In persistent $\mathrm{HCV}$ infection with MC, elevation of $\mathrm{Ig}$ is a general occurrence too. However, it is attributable to $\operatorname{IgG}$ and $\operatorname{IgM}$. The latter includes antibodies with rheumatoid factor (RF) activity, which are essential for the development of circulating, cryoprecipitable immune complexes. We found hypergammaglobulinemia is sustained by a peripheral expansion of IgMk+restriction indicating that a limited number of antigens drives their proliferation through BCR interaction. We have shown that IgM RF and their counterparts on the surface of bone marrow-resident monoclonal B cells react against a Fc-epitope of IgG and the HCV-NS3 protein. Purpose: Based on the above findings, we propose a model whereby $\mathrm{BCR}$, by binding the $\mathrm{Fc}$ of $\mathrm{IgM} / \mathrm{IgG} / \mathrm{HCVNS} 3$ immune complexes deprives FcgIIR of its natural ligand. This takes the brake off RF+ CD27+ B cell proliferation and promotes their selective accumulation which is otherwise prevented by increased apoptosis susceptibility in persistent $\mathrm{HCV}$ infection without MC.

Results and Conclusions: Immunoistochemical studies based on FcgIIR expression are ongoing. 


\section{LS 35}

\section{RECURRENT TRANSLOCATIONS INVOLVING THE MUMI} ONCOGENE LOCUS IN PERIPHERAL T-CELL LYMPHOMAS

*Andrew L. Feldman, Mark Law, Ellen D. Remstein, William R. Macon, Lori A. Erickson, Karen L. Grogg, Paul J. Kurtin, and Ahmet Dogan

\section{Mayo Clinic, Rochester, MN, USA}

Background: Oncogenes involved in recurrent chromosomal translocations serve as diagnostic markers and therapeutic targets in hematopoietic tumors. In contrast to myeloid and B-cell neoplasms, translocations in peripheral T-cell lymphomas (PTCLs) are poorly understood. MUM1 is a transcription factor involved in cellular activation and plasma cell differentiation. MUMI translocations exist in myeloma and some B-cell lymphomas, but have not been reported previously in PTCLs.

Methods: Based on a PTCL, unspecified (PTCL-U) with t(6;14)(p25; q11.2), we studied 169 PTCLs (including the index case) for MUM1 translocations. These included 23 angioimmunoblastic T-cell lymphomas, 72 PTCL-Us, 18 ALK-positive anaplastic large-cell lymphomas (ALCLs), 24 ALK-negative ALCLs, 14 cutaneous ALCLs (CALCLs), and 18 other PTCLs. Fluorescence in situ hybridization (FISH) was performed using a home-brew breakapart MUM1 probe. Additional studies included breakapart FISH for T-cell receptor-alpha (TCRA) translocations, dual-fusion FISH for MUM1/TCRA fusions, and MUM1 immunohistochemistry.

Results: We identified 12 PTCLs with MUM1 translocations. Two were PTCL-Us with $\mathrm{t}(6 ; 14)(\mathrm{p} 25 ; \mathrm{q} 11.2)$ and $M U M 1 / T C R A$ fusions by FISH. Both were cytotoxic, CD30-negative PTCL-Us involving bone marrow and skin. Eight of the remaining ten cases were C-ALCLs without TCRA rearrangements (57\% of C-ALCLs tested). A systemic ALK-negative ALCL and an additional CD30-positive PTCL-U also had MUM1 translocations. No ALK-positive ALCLs had MUM1 translocations. All cases with $M U M 1$ translocations were strongly MUM1-positive by immunohistochemistry.

Conclusions: Cytotoxic PTCL-Us involving bone marrow and skin and containing MUM1/TCRA translocations might represent a distinct clinicopathologic entity. Translocations involving MUM1 but not TCRA appear to occur predominantly in cutaneous ALCLs, representing the first recurrent translocation described in this disease. Detecting these translocations may be useful in lymphoma diagnosis. Due to its involvement in translocations, MUM1 may play an important biologic role in some PTCLs, and might represent a possible therapeutic target.

\section{LS 36}

\section{RESVERATROL ACTIVITY ON MCL CELL LIEN JEKO-1: A PROTEOMIC APPROACH}

Daniela Cecconi 1, Alice Parisi 2, Elena Bianchi 1, Claudia Parolini 2, Anna Maria Timperio 3, Lello Zolla 3, Marco Chilosi *2 and Alberto Zamò 2

1 University of Verona, Dipartimento Scientifico e Tecnologico, Laboratorio di Proteomica, Verona, Italy

2 University of Verona, Dipartimento di Patologia, Sezione di Anatomia Patologica, Verona, Italy

3 University of Tuscia, Dipartimento di Scienze Ambientali, Viterbo, Italy
Background. Therapies for mantle cell lymphoma (MCL) are clinically unsatisfactory, and the search for effective drugs in vitro might foster the evaluation of their activity in vivo. Resveratrol is a natural polyphenolic compound, showing a low toxicity profile, whose anti-cancer activity has been proven in several neoplasias, but not in MCL.

Purpose of the study. We have investigated the effects of resveratrol on the MCL cell line Jeko-1 using a combination of flow cytometry, western blotting and two-dimensional electrophoresis to identify the molecules involved in the induction of apoptosis and cell growth regulation.

Results. We show that resveratrol induces apoptosis in Jeko-1 cells and modulates several key molecules, including cyclin D1 (CCND1), p53 (TP53), p21 (CDKN1A), BCL2, BAX, Bcl XL (BCL2L1), caspase 9 (CASP9) and p27 (CDKN1B). By high-resolution 2DPAGE and nano-reverse phase-high performance liquid chromatography coupled with tandem mass spectrometry, we identified 32 differentially expressed proteins in response to resveratrol treatment that belong to important cell death related networks (including c-myc, NF-kB and the mitochondrial apoptotic pathway).

Conclusion. These findings may improve the understanding of mechanisms mediating the pro-apoptotic effects of resveratrol on MCL cells, and form the basis for its potential use as a therapeutic agent.

\section{LS 37}

\section{INTEGRATIVE GENOMIC ANALYSIS OF MANTLE CELL LYMPHOMA}

Lorena Di Lisio, Nerea Martinez, Cristina I. Gomez-Abad, *Margarita Sanchez-Beato, Gonzalo Gomez, Elena Rodriguez, Raquel Villuendas, Angel Carro, Bibiana Ferreira, Manuela Mollejo, Miguel A. Martinez, Juan C. Cigudosa, David G. Pisano, Miguel A. Piris

Lymphoma Group, Molecular Pathology Programme; Cytogenetics Unit, Human Genetics Programme; Bioinformatics Unit, Structural Biology and Biocomputing Programme, Spanish National Cancer Research Centre (CNIO), Madrid; Department of Pathology, Hospital Virgen de la Salud, Toledo; Department of Pathology, Hospital Doce de Octubre, Madrid. Spain

Mantle cell lymphoma pathogenesis remains partially unexplained. In the last few years, a significant role of microRNAs (miRNAs) in cancer has been recognized, whereby miRNAs play a role as tumor suppressors or oncogenes.

miRNA expression profiling (miREP) has been also described as a useful tool for classifying human tumors. Although several studies have been published for CLL, SMZL or DLBCL, little is known about the role of miRNA in others lymphoma types.

Here we explored the miREP in a set of MCL cases, reactive lymphoid tissue, isolated IgD+ cells and MCL-derived cell lines using the Agilent miRNA microarray platform. We also studied the gene expression profiles (GEP) and gene dosage of the same series using 44× Agilent Whole Human Genome platform for GEP and Agilent Human Genome CGH microarray for CGH.

MCL presents an unique miRNA signature revealing deregulation of 155 miRNAs in MCL in at least $50 \%$ of the samples, when compared with the average expression level of the controls. 
Combination of GEP and miREP data by informatics tools should reveal mechanisms of gene regulation by miRNAs, and could improve our understanding of the molecular pathogenesis of MCL. Actually, an integrated analysis of miREP and GEP data revealed an inverse correlation between the expression level of some miRNAs and their potential target genes, some of them constituting the characteristic gene expression signature of MCL. That is the case of overexpression of genes from NFkB pathway and downregulation of miR-148a, and miR-100, (which potentially regulate these genes); overexpression of several MAPK pathways members and downregulation of miR-133a, miR-31, miR-663; or overexpression of LYN and miR-1 or miR-132 downregulation. Downregulation of genes such as BCL6 are also correlated with increased expression of miR-363 and miR-765.

A correlation has also been done for chromosomal gain and losses with miREP data.

The miRNA signature in MCL thus contributes to explain the increased survival signaling, a key feature of MCL pathogenesis.

\section{$\underline{\text { LS } 38}$}

\section{NODAL INFLAMMATORY PSEUDOTUMOR CAUSED BY TREPONEMA PALLIDUM INFECTION.}

Incardona P, Ponzoni M, Chiodera C, Fisogni S, Legrenzi L, Lonardi $\mathrm{S}$, Facchetti $\mathrm{F}^{*}$.

Department of Pathology, Spedali Civili Brescia, Brescia, Italy; S.Raffaele Hospital, Milan, Italy; S.Anna Clinic, Brescia, Italy

Inflammatory pseudotumor of lymph nodes (IPT-LN) represents an unusual cause of lymphadenitis of unknown etiology. Upon the observation of a case of IPT-LN associated with Treponema pallidum (Tp) infection, we analysed a series of 9 IPT-LN and 9 extranodal IPT (spleen, 4 cases; lung, orbit, gut, skin and liver) for the presence of $\mathrm{Tp}$, using a polyclonal antibody anti-Tp (Biocare Medical, Concord, CA, USA). At the time of biopsy, none of the patients were suspected for luetic infection, nor specific serological tests were available. IPT$\mathrm{LN}$ areas extensively involved the nodal parenchyma in 4 cases, while they were focal in the remaining four cases. Capsular thickening and inflammation (6/9), venulitis (3/9), small granulomas (3/9), and follicular hyperplasia (7/9) were observed in the associated lymphoid parenchyma. Tp were detected in 4/9 cases of LN-IPT and in none of extranodal IPT. Tp were extremely abunbdant within the IPT areas and in the perivascular tissues in the surrounding parenchyma, while they were scattered within the capsule. In $\mathrm{Tp}+$ cases IPT was extensive in 2 and focal in 2 cases, and marked follicular hyperplasia was the single feature distinctively associated with these cases. Double immunostains for spirochetes and several leucocyte (CD3, CD20, CD138, CD163, S100) and vascular-associated (CD34, FactorVIIIRA, smooth muscle actin) antigens showed that Tp were mainly localized in the cytoplasm of macrophages and pericytes, but also occurred within endothelial cells and $\mathrm{S} 100+$ interdigitating cells. This study shows that a significant number of IPT-LN is associated with Tp infection. A spirochetal etiology should be suspected in all IPT-LN, independently from the extension of the lesions, especially when pronounced follicular hyperplasia is found. Infection by $\mathrm{Tp}$ of multiple cell types, including dendritic cells, supports data from in vitro studies and indicates that various mechanisms may be involved in the pathogenesis of the lesions.

\section{LS 39}

Activation of the Endoplasmic Reticulum (Er) Stress Associated Transcription Factor X-Box Binding Protein-1 (Xbp-1) Occurs in a Subset of Normal Germinal Center Cells and in Aggressive B-Cell Malignancies with Prognostic Implications.

Olga Balagué ${ }^{1}$, Ana Mozos ${ }^{1}$, Daniel Martinez ${ }^{1}$, Lluis Hernández ${ }^{1}$, Lluis Colomo ${ }^{1}$, Jose Luis Mate ${ }^{2}$, Julie Teruya-Feldstein ${ }^{3}$, Oscar Lin ${ }^{3}$, Elias Campo ${ }^{1}$, Armando López-Guillermo ${ }^{4}$, and Antonio Martinez ${ }^{1}$.

${ }^{1}$ Hematopathology Section, Laboratory of Pathology, Hospital Clínic, Institut d'Investigacions Biomèdiques August Pi i Sunyer (IDIBAPS), University of Barcelona, Barcelona, Spain. ${ }^{2}$ Department of Pathology, Hospital Universitari Germans Trias i Pujol, Autonomous University of Barcelona, Barcelona, Spain. ${ }^{3}$ Memorial Sloan-Kettering Cancer Center, Sloan-Kettering Institute, New York, USA. ${ }^{4}$ Hematology Department, Hospital Clínic

Background: Plasma cell differentiation and ER stress response converge in Xbp-1s activation. Xbp-1s is expressed in plasma cell tumors where acts as an oncogene and is targeted by the proteosome inhibitors. Little is known about Xbp1s expression in normal B-cells and B-NHL.

Purpose of the study: We analyze Xbp-1s expression by western blot, immunohistochemistry and RT-PCR. Localization in primary lymphoid tissues and its relationship with other main transcription factors related with B cell differentiation such as Pax-5, Bcl-6 and Irf4 were investigated. We also analyzed Xbp-1s activation in 24 cell lines, $271 \mathrm{~B}-\mathrm{NHL}$ and 39 plasma cell tumors.

Results: In reactive tissues, $\mathrm{Xbp}-1 \mathrm{~s}$ was found in nuclear extracts and nuclear localization was observed in plasma cells. Moreover, Xbp-1s was expressed in Irf-4+/Bcl-6-/Pax-5- germinal centre cells committed to plasma cell differentiation. In cell lines, Xbp-1s was broadly expressed independently of immunoglobulin secretion or plasmacytic differentiation.

None of the low grade lymphomas showed Xbp-1s expression which was found in $69 \%$ of myelomas, $48 \%$ of plasmablastic lymphomas and $28 \%$ of diffuse large B-cell lymphomas (DLBCL) regardless of germinal or post-germinal centre differentiation. Among DLBCL, $\mathrm{Xbp}-1 \mathrm{~s}$ was expressed in a subset of aggressive tumors with poor response to therapy and short overall survival (5-year OS: 29 vs. 53\%; $\mathrm{p}=0.01)$.

Conclusion: We demonstrate for the first time Xbp-1 activation in lymphomas, associated with aggressive subtypes and with plasma cell differentiation. Moreover, Xbpls expression is associated with a worse prognosis in DLBCL. Our findings suggest a role of Xbp-1 activation in the pathogenesis of the aggressive B-cell lymphomas. Therapies targeting the ER stress mediated activation of Xbp 1 may be use in the management of these neoplasms. 
$\underline{\operatorname{LS} 40}$

\section{A20 DELETION IS ASSOCIATED WITH GAIN AT THE TNFA/ $B / C$ LOCUS IN MALT LYMPHOMAS OF THE OCULAR ADNEXA, SALIVARY GLANDS AND THYROID}

*Estelle Chanudet, ${ }^{1}$ Hongtao Ye, ${ }^{1}$ Judith Ferry ${ }^{2}$, Chris M Bacon, ${ }^{1}$ Patrick Adam, ${ }^{3}$ Hans-Konrad Müller-Hermelink, ${ }^{3}$ John Radford, ${ }^{4}$ Stefano A Pileri, ${ }^{5}$ Koichi Ichimura, ${ }^{1}$ V. Peter Collins, ${ }^{1}$ Rifat A. Hamoudi, ${ }^{1}$ Andrew G Nicholson, ${ }^{6}$ Andrew C Wotherspoon, ${ }^{7}$ Peter G Isaacson, ${ }^{8}$ Ming-Qing $\mathrm{Du}^{1}$

${ }^{1}$ Department of Pathology, University of Cambridge, UK;

${ }^{2}$ Department of Pathology, Massachusetts General Hospital, Boston, MA;

${ }^{3}$ Institute of Pathology, University of Würzburg, Germany;

${ }^{4}$ Department of Medical Oncology, Christie Hospital, Manchester, UK;

${ }^{5}$ Unità Operativa di Emolinfopatologia - Università degli Studi di Bologna, Italy;

${ }^{6}$ Department of Histopathology, Royal Brompton Hospital, London, $\mathrm{UK}$;

${ }^{7}$ Department of Histopathology, Royal Marsden Hospital, London, UK;

${ }^{8}$ Department of Histopathology, University College London, UK.

Background: MALT lymphoma develops at various extranodal sites from a background of chronic inflammation or autoimmune disorders. The genetic basis of MALT lymphoma is largely unknown. Characteristic chromosomal translocations are frequently associated with gastric and pulmonary cases, but are rare at other sites. Purpose: Our study was designed to investigate and characterize genetic aberrations in MALT lymphomas of the ocular adnexa (33) and lung (25) by array-comparative genomic hybridization ( $\mathrm{CGH}$ ), followed by interphase fluorescence in situ hybridization (FISH).

Results: $1 \mathrm{Mb}$ genomic profiles revealed common genomic aberrations in ocular adnexal and pulmonary MALT lymphomas, but also recurrent 6q23 losses and 6p21.2-6p22.1 gains exclusive to ocular cases. High-resolution chromosome 6 tile-path array-CGH identified the gene for the NF-KB inhibitor A20 as the target of $6 \mathrm{q} 23.3$ deletion, and the TNFA/B/C locus as a putative target of $6 \mathrm{p} 21.2-22.1$ gain. Interphase FISH showed that $A 20$ deletion occurred preferentially in MALT lymphoma of the ocular adnexa $(8 / 42=19 \%)$, salivary gland $(2 / 24=8 \%)$, thyroid $(1 / 9=11 \%)$ and liver $(1 / 2)$, but not in the lung (26), stomach (45) and skin (13). Homozygous deletion was observed in 3 cases. $A 20$ deletion and $T N F A / B / C$ gain were significantly associated $(P<.001)$ and exclusively found in cases without characteristic translocation. In ocular cases, $A 20$ deletion was associated with concurrent involvement of the orbital soft tissue and the conjunctiva and/or lachrymal glands, or extra-ocular sites at diagnosis $(P=.007)$. $A 20$ deletion was also associated with a higher proportion of lymphoma relapse (67\% versus $37 \%)$ and a shorter relapse-free survival $(P=.033)$.

Conclusion: $A 20$ deletion and gain at the $T N F A / B / C$ locus may thus play an important role in the development of translocation negative MALT lymphomas, especially those arising from sites associated with auto-immunity.
LS 41

\section{ABERRANT COEXPRESSION OF SEVEN AUTOCRINE- AND PARACRINE- ACTIVATED RECEPTOR TYROSINE KINASES IN CLASSIC HODGKIN LYMPHOMA}

*Christoph Renné, Klaus Willenbrock, Martin-Leo Hansmann and Andreas Bräuninger

Senckenberg Institute for Pathology, University of Frankfurt, Germany

The pathogenesis of Hodgkin lymphoma (HL) is still largely unknown. Based on a search for pathogenetic mechanisms in global RNA expression data of several B-cell lymphoma and B-cell lymphoma cell lines, we analyzed the expression and activation of 7 receptor tyrosine kinases (RTKs) in classic HL.

Immunohistochemistry revealed that the RTKs PDGFRA, DDR2, EPHB1, RON, TRKB, TRKA and TIE1 were each expressed in Hodgkin/ Reed Sternberg (HRS) cells in $30 \%$ to $75 \%$ of HL patients but neither in non neoplastic B-cells nor in most B-non-Hodgkin lymphomas. Up to 7 RTKs were coexpressed, most prominently in Hodgkin lymphoma of the nodular sclerosis (ns) subtype.

Whereas no specific RTK expression pattern or relation of any RTK to specific clinical data of a group of cases was observed. RTK coexpression was significantly higher in EBV negative ns HL cases compared to both EBV positive and EBV negative mixed cellularity $\mathrm{HL}$ cases. In general coexpression of 3 and more RTKs was largely restricted to EBV negative HL cases.

Immunohistochemistry with phosphotyrosine specific antibodies revealed a general elevation of cellular phosphotyrosine content and exemplarily the activation of PDGFRA and TRKA/B in HRS cells. Immunohistochemistry and Western Blotting for RTK ligands indicated that DDR2 is likely activated in a paracrine fashion, PDGFRA and EPHB1 seem to be activated by autocrine mechanisms and TRKA combines autocrine and paracrine activation modi.

As the PDGFRA Inhibitor Imatinib and the TRK inhibitor K-252a decrease the survival of HRS cell lines it is possible that the inclusion of RTK inhibitors in therapeutic regimens of HL patients may thus be a novel option in HL treatment.

\section{LS 42}

\section{PLASMACYTOID DIFFERENTIATION OF B-CLL OCCURS IN PATIENTS WITH A NORMAL KARYOTYPE AS WELL AS WITH PROGNOSTICALLY RELEVANT CHROMOSOMAL ABERRATIONS}

Lukáš Plank $^{1 *}$, Tomáš Balhárek ${ }^{1}$, Denisa Ilenčíková ${ }^{2}$, Peter Szépe ${ }^{1}$, Alena Žákovičová ${ }^{2}$, Martin Čermák ${ }^{2}$, Martina Barthová ${ }^{1}$, Juraj Marcinek $^{1}$, Zuzana Kviatkovská ${ }^{1}$

${ }^{1}$ Department of Pathology, Comenius University Medical Faculty and Faculty Hospital in Martin, Slovakia

${ }^{2}$ Department of Oncological Genetics, National Cancer Institute, Bratislava, Slovakia

Background: Although it it possible to distinguish B-CLL with and without plasmacytoid differentiation (Pcoid+ and Pcoid-), the significance of this distinction remains unclear. 
Purpose: To correlate Pcoid differentiation with the presence of chromosomal abnormalities (CHA), incl. del11q22-23, del13q14, del17p13 and trisomy 12 .

Material and methods: 98 patients with hematologically verified BCLL and available CHA examinations. Pcoid was verified in bone marow and lymphoid tissue biopsies by reevaluation by two observers. CHA were analyzed in peripheral blood lymphocytes after $72 \mathrm{~h}$ cultivation by FISH using DNA probes (Vysis).

Results: Pcoid + was present in $39(40 \%)$ and absent in $59(60 \%)$ patients. From 27 cases (27.6\%) with a normal karyotype, 15 (55.6\%) were Pcoid+ a 12 (44.4\%) Pcoid-. CHA were detected in 71 cases (72.4\% of all), $24(33.8 \%)$ were Pcoid + and 47 (66.2\%) Pcoid-. Prognostically negative CHA (del11q, del17p, 12+, incl. del13q in combination) were found in 40 cases $(40.8 \%$ of all): $16(40 \%)$ were Pcoid + and $24(60 \%)$ Pcoid-. Isolated del13q considered to be a positive prognostic factor was identified in 31 cases (31.6\% of all): only 8 (25.8\%) were Pcoid+ and 23 (74.2\%) were Pcoid-.

Conclusions: In spite of the limited number of the cases our study demonstrates that B-CLL patients with a normal karyotype show similar frequency of either Pcoid + or Pcoid-. In contrast, Pcoid + is less frequent (33-40\% of the cases) in CLL with CHA generally and in the group of patients with prognostically negative CHA. The lowest proportion of Pcoid+ cases (approx. 1/4 of all) occurs in patients with isolated del13q. If Pcoid + should represent a sign of an advanced tumor cell differentiation than tendency to this differentiation seems to be lower in patients with CHA, incl. CHA considered to be prognostically negative

Supported by grants MZ SR 2005/12-MFN-04 and VEGA 1/4285/07.

\section{$\underline{\text { LS } 43}$}

\section{SNP ARRAY ANALYSIS REVEALS COPY NUMBER ALTERATIONS AND COPY NEUTRAL LOH IN MANTLE CELL LYMPHOMA AT HIGH RESOLUTION}

Elena Hartmann ${ }^{1}$, Itziar Salaverria ${ }^{2}$, Silvia Beà ${ }^{2}$, Andreas Zettl ${ }^{1}$, Pedro Jares ${ }^{2}$, Randy D. Gascoyne ${ }^{3}$, Wing C. Chan ${ }^{4}$, Dennis D. Weisenburger $^{4}$, Timothy C. Greiner ${ }^{4}$, Rita M. Braziel ${ }^{5}$, Lisa M. Rimsza $^{6}$, Jan Delabie ${ }^{7}$, Elaine S. Jaffe ${ }^{8}$, German Ott ${ }^{1,9}$, Hans Konrad Müller-Hermelink ${ }^{1}$, Andreas Rosenwald ${ }^{1}$, Elias Campo ${ }^{2}$

${ }^{1}$ Institute of Pathology, University of Würzburg, Würzburg, Germany ${ }^{2}$ Department of Pathology, Hospital Clínic, University of Barcelona, Barcelona, Spain

${ }^{3}$ British Columbia Cancer Agency, Vancouver, B.C., Canada

${ }^{4}$ Department of Pathology and Microbiology, University of Nebraska Medical Center, Omaha, NE, USA

${ }^{5}$ Department of Pathology, Oregon Health and Sciences University, Portland, OR, USA

${ }^{6}$ Department of Pathology, University of Arizona, Tucson, AZ, USA

${ }^{7}$ Norwegian Radium Hospital, Norway Hospital Clinic, Oslo, Norway

${ }^{8}$ Laboratory of Pathology, National Institutes of Health (NIH), Bethesda, MD, USA

${ }^{9}$ Institute of Pathology, Robert-Bosch Krankenhaus, Stuttgart, Germany

E. H. and I. S. contributed equally to this work. A.R. and E.C. are co-senior authors of this work.

Mantle cell lymphoma (MCL) is genetically characterized by the translocation $\mathrm{t}(11 ; 14)$. In addition, secondary chromosomal alterations likely contribute to MCL lymphomagenesis and influence the clinical course. There is increasing evidence that loss of heterozygosity $(\mathrm{LOH})$ without copy number $(\mathrm{CN})$ changes (copy neutral $\mathrm{LOH}$, cnLOH), might be an important alternative mechanism for tumor suppressor gene inactivation. Single Nucleotide Polymorphism (SNP) arrays allow - in addition to high resolution $\mathrm{CN}$ analyses and SNP genotyping - the detection of regions with $\mathrm{cnLOH}$. We analyzed $8 \mathrm{MCL}$ cell lines and 75 primary MCL cases (most of them previously analyzed by conventional metaphase CGH by Salaverria et al., JCO, 2007) with the Affymetrix $500 \mathrm{~K}$ array set using an unpaired analysis approach and compared the results with data obtained by conventional $\mathrm{CGH}$, in order to refine minimally altered regions and to determine the incidence of $\mathrm{cnLOH}$ in MCL. Furthermore, we investigated 5 CCND1(-) lymphoma cases, that showed pathohistological and gene expression features of conventional MCL. In an initial platform comparison including $\mathrm{CGH}$ and SNP array data, we found excellent agreement between the platforms regarding large $\mathrm{CN}$ alterations. However, SNP array analysis revealed a high number of additional $\mathrm{CN}$ alterations (median $=3$ using conventional $\mathrm{CGH}$ vs. median $=15$ using 500K SNP arrays for CCND1(+) cases), and allowed a more precise delineation of several altered regions. Moreover, large regions $(\geq 5 \mathrm{Mb}$ ) of $\mathrm{cnLOH}$ were found in $29 / 75(39 \%)$ of the CCND1 $(+)$ and $2 / 5(40 \%)$ of the CCND1(-) cases. The results demonstrate the capability of SNP array analysis for identifying $\mathrm{CN}$ alterations and $\mathrm{cnLOH}$ at high resolution in MCL cell lines as well as in primary tumor samples, and that $\mathrm{cnLOH}$ is a frequent phenomenon in MCL. Moreover, the results indicate that CCND1(+) and CCND1(-) MCL have similar profiles of secondary genetic alterations using this high density approach suggesting a closely related pathogenesis.

\section{LS 44}

\section{FOLLICULAR LYMPHOMAS WITH AND WITHOUT TRANSLOCATION t(14;18) DIFFER IN GENE EXPRESSION PROFILES AND GENETIC ALTERATIONS}

Ellen Leich ${ }^{1 *}$, Itziar Salaverria ${ }^{2}$, Silvia Bea ${ }^{2}$, Andreas Zettl ${ }^{1}$, Randy D. Gascoyne ${ }^{3}$, Wing-Chung $\mathrm{Chan}^{4}$, Rita M. Braziel ${ }^{5}$, Lisa M. Rimsza $^{6}$, Dennis D. Weisenburger ${ }^{4}$, Jan Delabie ${ }^{7}$, Elaine S. Jaffe ${ }^{8}$, Andrew Lister ${ }^{9}$, Andrew J. Norton ${ }^{9}$, Louis M. Staudt ${ }^{10}$, Elena M. Hartmann $^{1}$, Hans-Konrad Mueller-Hermelink ${ }^{1}$, Elias Campo ${ }^{2}$, German $\mathrm{Ott}^{1,11}$ and Andreas Rosenwald ${ }^{1}$

${ }^{1}$ Institute of Pathology, University of Wuerzburg, Wuerzburg, Germany; ${ }^{2}$ Department of Pathology, Hospital Clinic, Barcelona, Spain; ${ }^{3}$ British Columbia Cancer Agency, Vancouver, Canada; ${ }^{4}$ Department of Pathology and Microbiology, University of Nebraska Medical Center, Omaha, NE, USA; ${ }^{5}$ Southwest Oncology Group, Oregon Health and Science University, Portland, OR, USA; ${ }^{6}$ Department of Pathology, University of Arizona, Tucson, AZ, USA; ${ }^{7}$ Norwegian Radium Hospital, Oslo, Norway; ${ }^{8}$ Laboratory of Pathology, National Cancer Institute (NCI), Bethesda, MD, USA; ${ }^{9}$ Cancer Research UK, St. Bartholomews Hospital, London, United Kingdom; ${ }^{10}$ Metabolism Branch, National Cancer Institute (NCI), Bethesda, MD, USA and ${ }^{11}$ Institute of Clinical Pathology, Robert-Bosch-Krankenhaus, Stuttgart, Germany.

Follicular lymphoma (FL) is characterized by the translocation $t$ $(14 ; 18)(\mathrm{q} 32 ; \mathrm{q} 21)$. Approximately $15 \%$ of FL cases, however, lack this translocation by PCR and FISH analysis. The current study was 
designed to identify the frequency of $\mathrm{t}(14 ; 18)$-negative FL in a series of 164 FL cases graded 1-3a (Dave et al., NEJM 351:2159-69, 2004) and to compare genetic alterations and gene expression profiles between FL with and without $t(14 ; 18)$. Using PCR and tissue microarray-based FISH analysis $17 \mathrm{t}(14 ; 18)$-negative FL cases were identified in this series $(10 \%)$. In virtually all $t(14 ; 18)$-positive $\mathrm{FL}$ cases BCL2 expression was detected by immunohistochemistry (IHC), whereas 11 of the FL cases without a $\mathrm{t}(14 ; 18)$ were BCL2negative. Clinically, the $\mathrm{t}(14 ; 18)$-negative and -positive FL subgroups were similar regarding age and gender distribution as well as median survival times following different treatment regimens (without rituximab). Conventional metaphase comparative genomic hybridization $(\mathrm{CGH})$ analysis revealed alteration patterns that differed significantly between the $\mathrm{t}(14 ; 18)$-negative and -positive FL subgroups. In particular, FL cases with $t(14 ; 18)$ showed frequent gains of chromosome 18cen-q21 (27\%) which were absent in FL cases without $\mathrm{t}(14 ; 18)$. To compare gene expression between the two groups, we used gene set enrichment analysis (GSEA) which revealed an enrichment of GCB signatures in $\mathrm{t}(14 ; 18)$-positive cases and an enrichment of ABC-, NFKB- and Interferon signatures in $\mathrm{t}(14 ; 18)$ negative cases. Mutation analysis of the $\mathrm{IgV}_{\mathrm{H}}$ gene revealed ongoing somatic hypermutations in both the $t(14 ; 18)$-positive and the $t(14 ; 18)$ negative FL subgroup. Interestingly, in GeneScan analysis $\mathrm{t}(14 ; 18)-$ negative FL showed more often polyclonal or oligoclonal results. IHC analysis on an independent test set of FL cases showed strong CD10 expression in $100 \%$ of $\mathrm{t}(14 ; 18)$-positive cases and $86 \%$ of $\mathrm{t}(14 ; 18)$ negative cases with BCL2 expression, whereas CD10 expression was weak or absent in $\sim 34 \%$ of $\mathrm{t}(14 ; 18)$-negative FL without BCL2 expression. These results suggest that $\mathrm{t}(14 ; 18)$-negative FL differ from $\mathrm{t}(14 ; 18)$-positive $\mathrm{FL}$ at the molecular level.

\section{LS 45}

\section{HIGH CLINICAL IMPACT OF A COMBINED IMMUNOHISTOCHEMICAL OUTCOME PREDICTOR SCORE (CIOPS) IN DIFFUSE LARGE B CELL LYMPHOMA}

*S. Höller ${ }^{1,3}$, J. Kalla ${ }^{1}$. U, Mäder ${ }^{2}$, A. Lohr ${ }^{1}$, T. Katzenberger ${ }^{1}$, P. Went ${ }^{3}$, H-K. Müller-Hermelink ${ }^{1}$, A. Rosenwald ${ }^{1}$, G. Ott ${ }^{1,4}$

1: Institute of Pathology, University of Würzburg, Germany, 2: Tumorregitster, Universtity of Würzburg, Germany, 3: Instiute of Pathology, University of Basel, Switzerland 4.: Department of Clinical pathology, Robert-Bosch Krankenhaus, Stuttgart, Germany

The heterogenous group of DLBCL has been subclassified using gene expression profiling into three prognostically relevant subgroups, termed Germinal-center B-cell like DLBCL, Activated B-cell like DLBCL, and Mediastinal large B-cell lymphoma. This study was devised to clarify the role of immunophenotyping of paraffinembedded DLBCL specimens in the dissection into prognostically different risk groups.

145 large B-cell lymphomas (nodal and extranodal) were immunophenotyped using antibodies to CD10, BCL-6, MUM1p, BCL-2 and kappa and lambda light chains. Clinical data were collected from treating physicians. All patients had been treated with CHOP-like protocols, without Rituximab. The use of CD10, BCL-6 and MUM1p in analogy to the HANS algorithm resulted in two disease subgroups with prognostic relevance, a GC-like and a non GC-like subgroup (Kaplan Meier $\mathrm{p}=0,0027 \log$ rank). Adding data from BCL-2 and cIg, a CIOPS could be developed. For each unfavourable item (CD10-, BCL-6-, MUM1p+, BCL-2+, cIg+) one point was assigned, resulting in the delineation of four distinct prognostically relevant subgroups. A favourable CD10+, BCL-6+, MUM1p-, BCL-2- and cIg- group (CIOPS 1 (score 0): n=14), a CD10-, BCL-6-, MUM1p+, BCL-2+ and $\mathrm{cIg}+$ group (CIOPS 4 (score 5): $\mathrm{n}=8$ ) with dismal prognosis $(\mathrm{p}<0,0001 \log$ rank) and two significantly differing intermediate groups (CIOPS 2 (score 1 and 2): $n=73$, CIOPS 3 (score 3 and 4): $n=$ 50) $(p=0,0398 \log$ rank) (Fig. 1). Other immunohistochemical markers, like the expression of p53, FOXP1, or HLA-DR, or the Ki67 index, failed to identify prognostically relevant subgroups. DLBCL can be stratified into biologically and clinically relevant subgroups using immunohistochemistry in paraffin sections, like GCrelated and GC-unrelated subgroups. According to the newly designed CIOPS, these lymphomas can be divided into four subgroups with impressive statistically different overall survival (high risk, high/ intermediate, low/intermediate and low risk).

\section{$\underline{\text { LS } 46}$}

\section{BOB.1, CYCLIN E, MUM1P AND CD79A ARE HELPFUL MARKERS IN THE DIFFERENTIAL DIAGNOSIS OF CLASSICAL HODGKIN LYMPHOMA AND PRIMARY MEDIASTINAL B CELL LYMPHOMA}

*S. Höller, D. Zihler, E.C: Obermann, P. Hirschmann, S.A. Pileri*, S. Dirnhofer, A. Tzankov

Departments of Pathology, University Hospital Basel, Switzerland and University of Bologna, Italy*

Classical Hodgkin's lymphoma (cHL) and primary mediastinal B cell lymphoma (PMBCL) are closely related entities both at the immunohistochemical and molecular level. Even more, the recently established "mediastinal grey zone lymphoma", with its clinical, histological and immunohistochemical features, is situated in between cHL and PMBCL. Therefore, in daily practice, the differential diagnosis between $\mathrm{cHL}$ and PMBCL based on conventional histology and well established antibodies can be challenging.

In order to clarify which immunohistochemical expression profiles could be helpful to distinguish between these two entities, we analyzed 345 $\mathrm{cHL}$ and $51 \mathrm{PMBCL}$ on a validated tissue microarray platform with antibodies against BOB.1, CD15, CD20, CD23, CD30, CD79a, Cyclin E, LMP-1, MUM1p und Oct2. The positive predictive value (PPV), sensitivity and specificity of all markers were calculated using receiver operating characteristic curve (ROC) analysis and the Chi-square test. The number of evaluable cases for the different antibodies between 159 - 330 cases in the group of cHL and $39-50$ cases in the group of PMBCL. The markers with the highest PPV for cHL were: CD15 (100\%), Cyclin E (100\%), LMP-1 (100\%), MUM1p (98\%) and CD30 (97\%). A good sensitivity was only achieved by CD30 (93\%), Cyclin E (78\%), und MUM1p (67\%). In PMBCL, 7 out of 37 cases were also positive for $\mathrm{CD} 30$, which lead to a lower specificity considering cHL for this marker (81\%), compared to Cyclin E (100\%) and MUM1p (95\%). The antibodies with the highest PPV in PMCL were: CD23 (93\%), BOB.1 (73\%) and CD79a (58\%), with a good sensitivity in the case of BOB.1 (93\%) und CD79a (89\%).

The additional use of the immunohistochemical markers BOB.1, Cyclin E, MUM1p and CD79a may be helpful in the differential diagnosis of $\mathrm{CHL}$ and PMBCL. 


\section{$\underline{\text { LS } 47}$}

\section{THE IMMUNOPHENOTYPIC AND CYTOGENETIC FEATURES OF CD5-POSITIVE SPLENIC MARGINAL ZONE LYMPHOMA}

*Ellen D. Remstein, Mark E. Law, Dong Chen, William G. Morice, Curtis A. Hanson, Paul J. Kurtin, Andrew L. Feldman, Ahmet Dogan

Mayo Clinic, Rochester, MN USA

Background: Splenic marginal zone lymphoma (SMZL) may coexpress CD5 but the incidence and morphologic, immunophenotypic, and cytogenetic features of CD5+ SMZL are not well characterized.

Materials and Methods: Of 113 spleen specimens from patients with a primary SMZL diagnosis, 27 that were CD5-positive were further analyzed. We reviewed 19 flow cytometry histograms as well as karyotypes from 10 spleen and/or bone marrow specimens. Tissue microarrays (TMA) were constructed using paraffin-embedded tissue from all 27 SMZL specimens and immunohistochemistry (IHC) for cyclinD1, cyclinD2, cyclinD3, CDK6 and p27 and interphase FISH for $6 \mathrm{q}, 7 \mathrm{q} 32,11 \mathrm{q} 22-23$, centromere 12/12q15, 13q14, IGH-CCND1, BCL3, IGH, IGK and IGL were performed.

Results: All specimens had characteristic morphologic features of SMZL. By flow cytometry all 19 cases expressed moderate-bright CD20. 53\% expressed moderate-bright surface immunoglobulin (sIg) and partial CD5; 26\% expressed moderate-bright sIg and uniform CD5 (mantle cell lymphoma (MCL)-like); 16\% expressed dim sIg and partial $\mathrm{CD} 5$; and $5 \%$ expressed dim sIg, uniform CD5 and uniform CD23 (chronic lymphocytic leukemia (CLL)-like). By interphase FISH and/or karyotyping, $0 \%$ had del(6q); 12\% had del(7q); 20\% had $\operatorname{del}(11 \mathrm{q}) ; 8 \%$ had trisomy 12 and $21 \%$ had del(13q). Six cases $(23 \%)$ had translocations including $\mathrm{t}(6 ; 14)(\mathrm{p} 21 ; \mathrm{q} 32) / I G H-C C N D 3 \quad(\mathrm{n}=1)$, $\mathrm{t}(11 ; 14)(\mathrm{q} 23 ; \mathrm{q} 32) / I G H-?(\mathrm{n}=1),(9 ; 14)(\mathrm{p} 13 ; \mathrm{q} 32) / I G H-P A X 5 \quad(\mathrm{n}=1)$, $\mathrm{t}(2 ; 7)(\mathrm{p} 11.2 ; \mathrm{q} 22) / I G K-C D K 6(\mathrm{n}=2)$ and ?-CDK6 $(\mathrm{n}=1)$. By IHC, only the IGH-CCND3+ case was CCND3+, only the 3 CDK6 translocation-positive cases coexpressed both CDK6 and CCND2, and all cases were p27-positive and CCND1-negative.

Conclusions: Approximately $25 \%$ of SMZL coexpress CD5. By flow cytometry, CD5 is usually partially expressed but patterns mimicking MCL and CLL may occur. A minority of CD5+ SMZL have cytogenetic abnormalities such as del(11q), trisomy 12 and $\operatorname{del}(13 q)$. The incidence of $\operatorname{del}(7 q)$ in CD5+ SMZL $(12 \%)$ is slightly lower than that of SMZL overall, while $I G$ translocations are more common and partners often include genes of the CCND/CDK pathway other than CCND1.

\section{LS 48}

\section{IGVH ANALYSIS IN DIFFUSE LARGE B-CELL LYMPHOMA}

\author{
Mark A Catherwood, ${ }^{1,2}$ Lakshmi Venkatraman, ${ }^{3}$ Don Hull ${ }^{4}$, TCM Morris, ${ }^{1}$ \\ ${ }^{1}$ Haemato-Oncology, Department of Haematology, Belfast City \\ Hospital, Belfast, BT9 7AB. ${ }^{2}$ School of Biomedical Science, \\ University of Ulster, Coleraine, BT52 1SA, Northern Ireland. \\ ${ }^{3}$ Department of Histopathology, Royal Victoria Hospital, Belfast. \\ ${ }^{4}$ Department of Haematology, Craigavon Area Hospital
}

Background: Diffuse large B cell lymphoma (DLBCL), the commonest type of NHL is both clinically and biologically heterogeneous.
This was elucidated by the identification of 3 major subgroups termed, germinal centre B-cell like DLBCL (GCB-DLBCL), activated B cell-like DLBCL (ABC-DLBCL) and primary mediastinal DLBCL. Analysis of the variable region of the immunoglobulin heavy chain gene reveals information regarding the developmental stage at which malignant transformation has taken place.

Purpose: This study investigated the IGVH gene usage in a cohort of DLBCL (transformed follicular and de novo) to assess possible gene usage distinctions between GCB, non-GCB and mediastinal DLBCL as well as de novo and transformed DLBCL.

Methods: DNA was isolated from frozen tissue from 33 DLBCL (16 GC and 17 NGC) patients. PCR for complete (VDJH) rearrangements was performed using BIOMED-2 protocols. Germinal centre phenotype was defined by immunohistochemistry based on three markers CD10, BCL6 and MUM1.

Results: There were 24 de novo and 9 transformed DLBCL that could be categorized as GCB (16), non-GCB (17 including 3 mediastinal sub-type). Clonal productive rearrangements were detected in 30 cases and non-functional rearrangements in 3 cases. All cases showed somatic hypermutation (range 3-18\%). The $\mathrm{V}_{\mathrm{H}}$ genes were derived from 4 of the $7 \mathrm{~V}_{\mathrm{H}}$ families; most cases used either $\mathrm{V}_{\mathrm{H}} 3$ or $\mathrm{V}_{\mathrm{H}} 4$ gene segments. The IGVH usage was unbiased amongst GCB, non GCB and mediastinal subtypes; and in de novo and transformed cases. No correlation existed between survival and particular gene segment usage. 20/33 patients had been treated with Rituximab containing regimen. The average survival was 24 and 32 months respectively in the transformed and de novo DLBCL respectively. The overall survival was 20 and 35 months in the GCB and non-GCB sub-groups. Conclusion: Our findings show a random use of IGVH genes in the currently recognized clinical and biological sub-groups of DLBCL.

\section{LS 49}

\section{SUBMICROSCOPIC DELETIONS OF CHROMOSOME 22q11 ARE FREQUENT IN CHRONIC LYMPHOCYTIC LEUKEMIA}

*Shelly R. Gunn, ${ }^{1,2}$ Lynne V. Abruzzo, ${ }^{3}$ David W. Bahler, ${ }^{4}$ Clemens H. M. Mellink, ${ }^{5}$ and Ryan S. Robetorye ${ }^{1}$

${ }^{1}$ Department of Pathology, The University of Texas Health Science Center at San Antonio, San Antonio, TX; ${ }^{2}$ Combimatrix Molecular Diagnostics Inc., Irvine, CA; ${ }^{3}$ Department of Hematopathology, University of Texas M. D. Anderson Cancer Center, Houston, TX; ${ }^{4}$ Department of Pathology and ARUP Institute for Clinical and Experimental Pathology, University of Utah, Salt Lake City, UT; and ${ }^{5}$ Department of Clinical Genetics, Academic Medical Center, Amsterdam, the Netherlands

Background: Genomic aberrations have increasingly gained attention as prognostic markers in chronic lymphocytic leukemia (CLL). Fluorescence in situ hybridization (FISH) has improved the detection rate of genomic alterations in CLL from approximately 50\% using conventional cytogenetics to greater than $80 \%$. More recently, array comparative genomic hybridization (array $\mathrm{CGH}$ ) has gained popularity as a clinical tool that can be applied to detect genomic gains and losses of prognostic importance in CLL. In this study, bacterial artificial chromosome array-based array $\mathrm{CGH}$ was evaluated as a tool for the detection of recurrent genomic imbalances currently assayed by FISH panels in 193 cases of CLL.

Results: Detection rates of clinically relevant chromosomal abnormalities involving losses of $11 \mathrm{q} 23,13 \mathrm{q} 14,17 \mathrm{p} 13$, and whole 
chromosome 12 gains were consistent with previously reported results. Unexpectedly, submicroscopic deletions of chromosome 22 q11 were observed in 28 cases $(15 \%)$, with five of the cases exhibiting bi-allelic deletions. Interestingly, one mono-allelic deletion case and one bi-allelic deletion case also exhibited concomitant gains of whole chromosome 22 . The frequency of $22 \mathrm{q} 11$ deletions in this study was second only to loss of the $13 \mathrm{q} 14$ region, the most commonly detected recurrent chromosomal aberration in CLL. Breakpoint mapping using oligonucleotide-based array CGH analysis of selected cases showed that the 22q11 deleted regions ranged in size from $\sim 0.3 \mathrm{Mb}$ up to $\sim 0.9 \mathrm{Mb}$, thereby defining a new subgroup of CLL at the genomic level.

Conclusions: We have demonstrated that mono- and bi-allelic 22q11 deletions are relatively frequent in CLL, occurring in approximately $15 \%$ of cases. Further studies are needed to assess the prognostic significance of these submicroscopic deletions and to determine whether these alterations might predispose to the development of CLL, contribute to the pathogenesis of CLL, or both.

\section{$\underline{\text { LS } 50}$}

\section{PRIMARY MARGINAL ZONE B-CELL LYMPHOMA OF THE CENTRAL NERVOUS SYSTEM (MZL-CNS) ASSOCIATED WITH CHLAMYDOPHILA PSITTACI (CP) INFECTION}

M. Ponzoni ${ }^{1,2}$, L. Poliani $^{3}$, M.G. Cangi ${ }^{1}$, R. Dolcetti ${ }^{4}$, A.J.M. Ferreri ${ }^{5}$, E. Dal $\mathrm{Cin}^{1}$, F. Bonetti ${ }^{3}$, E. Pasini ${ }^{4}$, C. Doglioni ${ }^{1,2}$, G. Rossi ${ }^{6}$, F. Facchetti ${ }^{3}$

${ }^{1}$ Pathology Unit and ${ }^{5}$ Medical Oncology Unit, Department of Oncology, ${ }^{2}$ Unit of Lymphoid Malignancies, San Raffaele Scientific Institute, ${ }^{3}$ Department of Pathology, University of Brescia, ${ }^{4}$ Department of Pre-Clinical Research, Centro di Riferimento Oncologico, Aviano, ${ }^{6}$ Department of Hematology, Spedali Civili, Brescia, Italy

Background: The association between non-Hodgkin lymphomas and $\mathrm{Cp}$ infection has been reported, with variable geographic distribution, mainly in ocular adnexal lymphomas (OAL). Reports about the occurrence of $\mathrm{Cp}$ infection in non-OAL suggest an association with lymphomas arising in organs exposed to air-transported antigens. No data are available about the occurrence of $\mathrm{Cp}$ infection in brain lymphomas. Purpose of the study: We report the occurrence of $\mathrm{Cp}$ infection in a patient with MZL-CNS. The assessment of Cp infection was made either with PCR-based methods and immunohistochemistry.

Results: A 44-years old woman with a 20-year long history of chronic cephalea complained with cephalalgia and hyperpyrexia. A brain CT scan showing a cerebral lesion (right-empisphere) was followed by surgical resection; histopathological examination concluded for MZL. $\mathrm{Cp}$ was detected in tumoral tissue by two different PCR (TETR for 16S-23S spacer region and Gro-El for Hsp-60 gene), followed by direct sequencing and by immunohistochemistry directed against genus-specific chlamydial lypopolisaccharide. TETR-PCR did not detect $\mathrm{Cp}$ in patient's peripheral blood mononuclear cells.

Conclusion: This is the first case of MZL-CNS associated with Cp infection. This observation warrants further investigation in large series of brain lymphomas including either low-grade and high-grade histotypes, in order to highlight potential pathogenetic and therapeutic implications.
LS 51

\section{HHV-8-POSITIVE ENDOTHELIAL PROLIFERATIONS: REPORT OF FIVE CASES AND DISCUSSION OF THE MINIMUM DIAGNOSTIC CRITERIA FOR KAPOSI'S SARCOMA.}

*Elizabeth J. Soilleux ${ }^{1}$, Kevin Gatter ${ }^{1}$, Andrew Rainey ${ }^{2}$, George Philip $^{2}$, Sebastian Lucas ${ }^{3}$

${ }^{1}$ Nuffield Department of Clinical Laboratory Sciences, Oxford University, John Radcliffe Hospital, Oxford OX3 9DU U.K.

${ }^{2}$ Department of Histopathology, The Royal Sussex County Hospital, Brighton BN2 5BE U.K.

${ }^{3}$ Department of Histopathology, St Thomas' Hospital, London SE1 7EH U.K.

Background: Kaposi's sarcoma is a vascular neoplasm that arises in association with HHV-8 infection, generally, but not exclusively, in HIV-positive patients.

Purpose: We describe five recent HHV-8-positive endothelial proliferations in HIV-positive patients that fail to efface tissue architecture and we consider the minimum morphological and immunophenotypical features required for a diagnosis of Kaposi's sarcoma.

Results: We present the case of a 40 year old HIV-positive man, admitted with fever, who died 10 days later with presumptive diagnoses of Listeria monocytogenes meningitis and active chronic hepatitis B, although no autopsy was performed, due to the risk of infection. A lymph node biopsy was performed to investigate widespread low volume lymphadenopathy. This demonstrated follicular lysis, in keeping with HIV, and focally prominent sinus endothelial proliferation. These endothelial cells immunostained positively for HHV-8 and the vascular markers, CD31 and CD34, in addition to the lymphatic endothelial markers LYVE-1 and DC-SIGN/ DC-SIGNR. The endothelial proliferation did not efface the lymph node architecture or extend beyond the lymph node capsule. A liver biopsy failed to demonstrate hepatic Kaposi's sarcoma.

We describe a further four cases of HHV-8-positive endothelial proliferation, occurring in HIV-positive male patients aged between 43 and 74 years, three in the skin and one in the liver. In each case, the endothelial proliferation fails to efface the architecture of the tissue and does not show significant dissection of collagen. We demonstrate the morphology and immunphenotype of these proliferations.

Conclusions: These five cases raise questions about whether all HHV8-positive endothelial proliferations should be regarded as Kaposi's sarcoma. Philosophically, one might imagine a biological continuum between HHV-8 infected endothelial cells and an obvious neoplasm composed of malignant endothelial cells. No clear minimum diagnostic criteria for Kaposi's sarcoma are currently available. We discuss possible diagnostic criteria that might be used. 


\section{LS 52}

\section{LYMPHOCYTE-RICH CLASSICAL HODGKIN LYMPHOMA.} A TUMOR DISTINGUISHED BY THE MICROENVIRONMENT

Syong H. Nam-Cha ${ }^{1,2}$, Santiago Montes-Moreno ${ }^{1}$, Maria T Salcedo, Juan F. Garcia ${ }^{1}$,Miguel A. Piris ${ }^{1}$

1. Lymphoma Group, Molecular Pathology Programme, Spanish National Cancer Research Centre (CNIO), Madrid, Spain

2. Department of Pathology, Complejo Hospitalario Universitario de Albacete, Albacete, Spain

The existence, diagnostic features, biological and clinical relevance of de diagnosis of Lymphocyte rich classical Hodgkin lymphoma (LRCHL) is still a debatable issue. Here we have performed a comparative marker analysis of LRCHL (16 cases), Nodular Lymphocyte Predominance Hodgkin Lymphoma (NLPHL) (68 cases) and other classic Hodgkin lymphoma (CHL) (60 cases), using tissue microarrrays. Markers selected for the study were focused in three different fields: B-cell lineage and transcription programme (OCT.1, OCT.2, BOB.1, BCL-6, PAX-5, GCET-1, KLHL-6, BLIMP-1), NFKB signaling pathway (REL-B, C-REL, TRAF-1, p50, MUM-1) and T-cell microenvironment (CD3, CD57, PD-1, CXCL-13, CD10, BCL-6).

$\mathrm{CHL}$ and NLPHL show differences in all the analyzed programs. Thus CHL has stronger expression of NF-kB markers, a weaker expression of the GC program and absence of follicular T-cell microenvironment.

LRCHL cases display intermediate features between CHL and NLPHL. Thus, there is a more frequent expression of B-cell transcription factors like OCT.1, OCT.2, BOB.1 and BCL6 than in CHL. A follicular T-cell microenvironment was also identified in 50\% of the LRCHL. Expression of NF-kB markers was seen in a frequency comparable to CHL.

In conclusion, Lymphocyte-rich classical Hodgkin lymphoma (LRCHL) seem to be characterized by Hodgkin/Reed-Sternberg cells (HRS) with a classical HRS cell immunophenotype, strong expression of B-cell transcription factors and a T-cell background similar to NLPHL.

\section{$\underline{\text { LS } 53}$}

\section{RT-PCR BASED PREDICTIVE MODEL OF CLINICAL OUTCOME FOR ADVANCED HODGKIN LYMPHOMA PATIENTS: INTEGRATING TUMOR AND MICROENVIRONMENT SIGNATURES}

*Juan F. García ${ }^{1,2}$, Beatriz Sánchez-Espiridion ${ }^{2}$, Carlos Montalbán ${ }^{3}$, Rafael Ramos ${ }^{4}$, Jose Rodriguez ${ }^{4}$, Francisco Mestre ${ }^{4}$, Araceli Cánovas $^{5}$, Carmen Camarero ${ }^{5}$, Antonio Salar ${ }^{6}$, Sergio Serrano ${ }^{6}$, Concepción Rayón ${ }^{7}$, Manuel F. Fresno ${ }^{7}$, Manuel M. Morente ${ }^{2}$, and Miguel A. Piris ${ }^{2}$. In behalf of the Spanish Hodgkin Lymphoma Study Group.

MD Anderson, International, Madrid (1); Centro Nacional de Investigaciones Oncológicas (CNIO), Madrid (2); Hospital Ramón y Cajal, Madrid (3); Hospital Son Dureta, Palma de Mallorca (4); Hospital de Cruces, Baracaldo (5); Hospital del Mar, Barcelona (6); Hospital Central de Asturias, Oviedo (7). SPAIN

Current predictive systems, based on clinical and analytical parameters, fail to accurately identify a significant fraction of advanced classical Hodgkin lymphoma (cHL) patients with short failure free survival (FFS).
Transcriptional analysis has identified genes and pathways associated with clinical failure, but the biological relevance and clinical applicability of these data are still pending of further development. Robust molecular techniques for the identification of biological processes associated with treatment response are necessary to develop new predictive tools.

We designed a quantitative RT-PCR based assay to be applied to routine formalin-fixed paraffin-embedded samples (FFPE), integrating genes known to be expressed either by the tumor cells and their reactive microenvironment, and related with clinical response to adriamycinbased chemotherapy. Using this approach, 138 FFPE tumor samples were analyzed and adequate RT-PCR profiles were obtained in all cases. Normalized expression levels $(\Delta \mathrm{Ct})$ of individual genes vary considerably among samples. However, consistent with previous results most of these genes were, on average, overexpressed in the unfavorable group. The results of the assay can be combined in a logistic regression model integrating pathways associated with treatment response. The final model can predict treatment response with an overall accuracy of $74,6 \%$ (chi-square $54,55, \mathrm{p}=0,004$ ).

Predicted probabilities identify two different risk groups associated with clinical outcome (5-years FFS probabilities $73,1 \%$ vs $20,8 \%$, log rank statistic $20,76, \mathrm{p}<0.000$; OS probabilities 89,5 vs. $54.5 \%$, log rank statistic $42,10, p<0.000)$. Moreover, this biological model is independent and complementary of the conventional International Prognostic Score using multivariate Cox proportional hazards analysis. In summary, we have demonstrated the potential prognostic capacity of our assay finding a positive correlation between gene expression and treatment response. These results could pave the way into a more comprehensive analysis of larger series of cHL patients, allowing the identification of subsets of patients who might benefit from alternate approaches.

\section{LS 54}

\section{ABERRANT PDGFRA SIGNALLING IS SUSTAINED BY AN AUTOCRINE LOOP IN PERIPHERAL T-CELL LYMPHOMA NOT OTHERWISE SPECIFIED}

*Piccaluga Pier Paolo ${ }^{1}$, Rossi Maura ${ }^{1}$, Esposito Aurora ${ }^{1}$, Tazzari Pier Luigi $^{2}$, Ricci Francesca ${ }^{2}$, Agostinelli Claudio ${ }^{1}$, Righi Simona ${ }^{1}$, Gazzola Anna ${ }^{1}$, Went Philip ${ }^{3}$, Hartmann Sylvia ${ }^{4}$, Hansmann Martin $\mathrm{L}^{4}$, Alviano Francesco ${ }^{5}$, Bagnara Gian Paolo ${ }^{5}$, Zinzani Pier Luigi ${ }^{1}$, Pileri Stefano $\mathrm{A}^{1}$

${ }^{1}$ Department of Hematology and Oncology "L. and A. Seràgnoli", Hematology and Ematopathology Sections, Molecular Pathology Laboratory, S. Orsola-Malpighi Hospital, University of Bologna, Bologna, Italy

${ }^{2}$ Service of Transfusion Medicine, S.Orsola-Malpighi Hospital, Bologna

${ }^{3}$ Institute of Pathology, Basel University, Basel, Switzerland

${ }^{4}$ Department of Pathology, University of Frankfurt, Frankfurt, Germany ${ }^{5}$ Department of Histology, Embriology and Applied Biology, University of Bologna, Italy

Background. Peripheral T-cell lymphoma not otherwise specified (PTCL/NOS) is the most common T-cell lymphoma; however, it remains a complex entity showing great variety regarding morphology, immunophenotype and clinical behaviour. Recently, gene 
expression profiling (GEP) revealed PDGFRA as possible PTCLassociated molecule, by nominating it as potential therapeutic target. Purpose of the study. We investigated the possible determinants of PDGFRA activity in PTCL/NOS.

Results. First, we performed GEP of 28 PTCLs/NOS and 20 samples of normal T-cell subpopulations, by using the Affymetrix HG-U133 2.0 plus microarray. GEP showed over-expression of PDGFRA in all tested PTCLs/NOS. Second, immunohistochemistry (IHC), performed on tissue-microarrays (TMAs) including 145 PTCLs/NOS, confirmed the expression of PDGFRA and its activated (phosphorilated) form, pPDGFRA, in most if not all instances. The PDGFRA locus (4q1.14q1.3) was then studied by FISH and wide-genome SNPs analysis (Affymetrix 500K Array), while direct sequencing of all PDGFRA exons and introns, as well as of the promoter region was performed in 90 cases. These approaches showed preserved integrity of the PDGRA locus. We then studied PDGF-A, PDGF-B and PDGF-C on both TMAs by IHC and supernatants from PTCL/NOS cell cultures by ELISA. Of note, they turned out to be expressed and secreted by PTCL/NOS cells. Finally, in order to verify the hypothesis of an autocrine loop, we tested whether the remotion of PDGF ligands from the supernatant of cultured PTCL/NOS cells determined PDGFRA dephosphorilation. In particular, the expression of PDGFRA and pPDGFRA was assessed by flow-citometry in PTCL/NOS cultured cells before and after the exposure to an anti-PDGF ligand neutralizing antibody (R\&B System). Notably, $48 \mathrm{~h}$ after the exposure to $20 \mu \mathrm{g}$ of anti-PDGF antibody, we already appreciated a reduction of PDGFRA phosphorilation up to $75 \%$.

Conclusions. Taken together, our data demonstrate that PDGFRA activity is sustained by an autocrine loop in PTCL/NOS.

\section{$\underline{\text { LS } 55}$}

Please see LS 37

\section{LS 56}

\section{PROLIFERATION CENTRES IN CHRONIC LYMPHOCYTIC LEUKEMIA CONTAINS SPECIFIC DENDRITIC, T-CELL AND B-CELL SUBPOPULATIONS INVOLVED IN THE REGULATION OF B-CELL PROLIFERATION AND NF-KB ACTIVATION.}

*SM Rodriguez-Pinilla; B Herreros, I Castillo, P Algara, S Montes-Moreno, R Diaz de Otazu, M Pérez Guillermo, MJ Mestre, C Bellas, MA Piris

Proliferation centres (PCs) are a distinctive tissue finding in Chronic Lymphocytic Leukaemia, of unknown significance. Cell composition analysis of proliferation centres may clarify the functional relevance of this tissue compartment.

An immunohistochemical analysis of a series of lymph nodes in CLL cases has allowed identifying specific cell subpopulations present in PCs, such as:

- Dendritic cells, positive for STAT1 and actin, negative for other markers of interdigitating and follicular dendritic cells.

- T-cells coexpressing CD4 and CD25, partially expressing PD1, negative for FoxP3.
This may account for the increased expression by the B-cells occupying the PCs of markers reporting for:

- Proliferation : Ki67

- Apoptosis: Low Bcl2, High MCL1 and BIRC5

- NF-kB: IkB $\alpha-p$, MUM1, TRAF1 and nuclear NFKB1.

- $\quad$ BCR signalling: CD40, ZAP70.

- Somatic Hypermutation: DP1, CDK7.

Nuclear expression of NFKB1 (p50) confirms NF-kB activation takes place in PCs. Interestingly, PC B-cells exhibit a strong expression of cytoplasmic and nuclear TRAF1, a molecule described to be induced in response to $\mathrm{CD} 40$ signalling by B-cells.

PC B-cells also display increased expression of SHM markers such as DP1 and CDK7.

PCs seem to constitute a distinctive tissue compartment, where NF-kB activation could take place in response to signals from specific dendritic and T-cell subpopulations.

\section{LS 57}

\section{INTRAFOLLICULAR NEOPLASIA (IN SITU FOLLICULAR LYMPHOMA). A REVIEW OF A SERIES OF 15 CASES.}

*Yolanda Castro, Santiago Montes-Moreno, Marien Castillo, Lidia Sanchez-Verde, Javier Menarguez, Juan Cruz Cigudosa, Miguel Angel Piris.

In situ localization of Follicular Lymphoma (FL) was described in 2002 by E. Jaffe and coworkers (Blood. 2002;99:3376-3382), and proposed as an early step either in the colonization of Germinal Centres by FL or in the development of FL.

Here we review the features of a series of 15 cases of lymph node biopsies with the diagnosis of in situ localization of FL. We have investigated the presence of $t(14 ; 18)$ involving $b c l 2$ gene either by PCR and FISH and we have correlated the findings with the clinical features of the patients.

Three main groups of patients were identified:

- Seven patients had no evidence of FL after conventional staging procedures at the moment of diagnosis of in situ FL. Only one of this cases developed FL after 15 monts of follow-up. The remaining patients did not develop other evidence of FL in the follow up (median time of 7 months, interval 3-25 months).

- Six patients had concurrent FL at the moment of diagnosis of in $s i t u \mathrm{FL}$, as revealed in the clinical staging. Interestingly these two groups could be distinguished using a quantitative score of germinal centre infiltration that showed that cases showing FL colonization were characterized by a higher score.

- A third group of patients consisted of two unusual cases that presented the association of in situ FL with Classical Hodgkin lymphoma. Of these, one patient showed established FL after staging procedures. The other patient has no evidence of FL after a follow up of 3 months.

With regard to molecular studies, in 13 patients $(86 \%)$ bcl 2 translocations were demonstrated, either by FISH or PCR. Either technique alone was positive only in a $60 \%$ of the cases, showing the need of a complementary approach. Interestingly one case of in situ FL showed amplification of bcl2, and infrequent event in FL. 
In conclusion so called in situ FL may represent three different conditions, with some cases apparently not requiring any additional therapy. The relatively high incidence of established FL in some cases with higher degrees on infiltration of the germinal centres suggests the need of performing conventional staging procedures to discard the presence of full blown FL. However, the absence of such relationship in a substantial part of the cases and the association with other lymphomas such as HL suggests that some cases may represent a particular disease entity whose role as preneoplastic lesion remains to be fully elucidated.

\section{LS 58}

\section{DEREGULATED EXPRESSION OF THE POLYCOMB- GROUP PROTEIN SUZ12 TARGETS GENES ASSOCIATED WITH TUMORAL TRANSFORMATION IN MANTLE CELL LYMPHOMAS}

Daniel Martín-Pérez, Esther Sánchez, Lorena Maestre, Javier Suela, Lorena di Lisio, Nerea Martínez, Miguel A. Piris, *Margarita Sánchez-Beato

Lymphoma Group, Molecular Pathology Programme, Monoclonal Antibodies Unit, Biotechnology Programme, and Cytogenetics Unit, Human Genetics Programme, Spanish National Cancer Research Centre (CNIO), Madrid, Spain. Department of Pathology, Hospital "Nuestra Señora del Prado", Talavera de la Reina-Toledo, Spain

Polycomb genes are transcriptional regulators with central roles in cell fate decision, cell renewal, and development, and have been implicated in cancer development. This is the case for EZH2, a member of the PRC2 complex with histone methyltransferase activity and involved in progression of prostatic cancer and in neoplastic transformation of breast epithelial cells. SUZ12, other important member of this complex, has been also shown to be implicated in stem cell development and maintenance.

Although some studies have shown over-expression of SUZ12 in certain tumors and SUZ12 locus translocation in endometrial stromal tumors, its real relevance in human cancer has not yet been established.

We have generated a SUZ12 monoclonal antibody (220A) and analyzed the expression of SUZ12 and EZH2 in reactive lymphoid tissue and in a set of 94 mantle cell lymphomas (MCL) samples. The results showed that whereas SUZ12 is detected exclusively in germinal centre proliferating cells in reactive lymphoid tissue, it is overexpressed in $56 \%$ of MCL cases. We also demonstrated that SUZ12 locus has increased copy number in $8 \%$ of MCL analyzed cases. These results suggest that in MCL SUZ12 could have an oncogenic/tumorigenic role independently of EZH2.

To further demonstrate the relevance of SUZ12 in MCL pathogenesis we performed functional assays silencing SUZ12 and we also integrated multiple genomic data combining genome-wide location analysis in MCL-derived cell lines, with gene expression profiling in both the SUZ12-silenced cell lines and MCL tumoral samples.

We demonstrate that SUZ12 loss compromise cell viability, identifying a set of SUZ12-target genes relevant in MCL pathogenesis, including genes involved in DNA-repair, apoptosis, NFkB pathway, or cell cycle control.

Therefore, we propose that the abnormal expression of SUZ12 may account for some of the still unexplained MCL features, such as abnormal DNA repair, increased resistance to apoptosis and others.

\section{LS 59}

\section{AN INCREASED AMOUNT OF PD-1+ TUMOR-INFILTRATING LYMPHOCYTES IN CLASSICAL HODGKIN LYMPHOMA IS ASSOCIATED WITH POORER OVERALL SURVIVAL}

\author{
S. Muenst, *S. Höller, S. Dirnhofer, A. Tzankov
}

Background: Programmed Death-1 (PD-1), a member of the CD28 costimulatory receptor superfamily, has an inhibitory function on T-cell activity. It plays a role in cellular immune response as well as peripheral tolerance and is physiologically expressed by germinal center-associated helper T-cells in reactive lymphoid tissues. The distribution of PD-1+ lymphocytes in the tumor microenvironment of Hodgkin Lymphoma (HL) is not random and can serve as a diagnostic marker.

Purpose: We aimed to measure the amount of PD-1+ lymphocytes in HL and to correlate this finding with the remaining background lymphocyte populations and with known biological and clinical key data, based on a tissue microarray (TMA) platform encompassing 280 cases of classical HL (cHL) and 3 cases of nodular lymphocyte predominant HL (NLPHL). Prognostic cut-off scores were determined by receiver-operating curve (ROC) analysis.

Results: The amount of PD-1+ tumor-infiltrating lymphocytes in 189 evaluable cases was median 8 , mean 76 cells/core and median 27 , mean 269 cells/square $\mathrm{mm}$, respectively, and was higher in lymphocyte-rich (LR) cHL and lower in the mixed cellular (MC) variant. Rimming of tumor cells by PD- $1+$ cells was observed in all evaluable cases of NLPHL but only in 1\% of cHL, particularly in LR and MC cHL. The PD-1+ cell amount was lower in cHL cases with 9p24 gains $\left(P D-1\right.$ ligand 2 locus; $\left.\mathrm{p}^{\text {Mann-Whitney }}=0.035\right)$ and in cases with higher amounts of FOXP3 + regulatory T-cells $\left(\mathrm{p}^{\text {Pearson }}=0.063\right)$. An increased amount of PD-1+ tumor-infiltrating lymphocytes above the ROCdetermined prognostic cut-off score of 23 cells/square $\mathrm{mm}$, turned to be a stage-independent negative prognostic factor considering overall survival $\left(\mathrm{p}^{\text {Kaplan-Meier }}=0.005\right)$.

Conclusion: An increased amount of PD-1+lymphocytes is a novel negative prognostic marker for overall survival in HL, as opposed to the amount of FOXP3+ regulatory T-cells. Along with the latter, PD1+ cells might represent important lymphoma/host microenvironment modulators.

\section{$\underline{\operatorname{LS} 60}$}

\section{MICRORNA PROFILE OF DLBCL. IDENTIFICATION OF MIRNAS SIGNATURES RELATED WITH CLINICAL OUTCOME IN R-CHOP TREATED PATIENTS.}

*Santiago Montes-Moreno, Nerea Martinez, Anabel Saez, Carlos Montalbán, Helena Rodriguez, Lydia Sanchez, A Lopez, MC RuizMarcellan, M Canales, P. Mazorra, E. Conde, M. Cruz, M. Mollejo, MA. Martinez, C. Grande, MA Piris.

Background: MicroRNAs have recently been shown to play a role in malignant transformation. Few reports have shown the relationship between isolated miRNAs with specific DLBCL phenotypes or prognosis. However a large scale miRNA profile of DLBCL is lacking. In this study we examined microarray microRNA expression profiles in a series of 36 patients with DLBCL uniformly treated with R-CHOP. Microarray microRNA expression profiles were correlated with clinical outcome (Lymphoma Specific Survival) by means of SignS 
(Signatures for Survival Data) analysis software. Differential expression profiles among immunohistochemically defined subtypes (GC vs nonGC, according to Hans et al. Blood. 2004;103:275-282) and cases associated with and without EBV infection were also identified.

Results: A set of 61 miRNAs showed a relationship with survival time with significant $\mathrm{p}$ values $(<0.05)$ according to a single gene Cox model. Of these, a selection of 25 miRNAs was identified as a miRNA signature that was inversely associated with LSS ( $p$ value 0.046 ).

Differential miRNA expression analysis among subtypes of EBVnegative DLBCL cases identified 15 differentially expressed miRNAs (8 upregulated in GC: miR9, miR9*, miR28, miR129, miR138, miR151, miR196a, miR196b; and 7 upregulated in nonGC cases: miR141, miR144, miR200c, miR221, miR222, miR31, miR375).

A set of 87 miRNAs were identified as differentially expressed among EBV positive or negative DLBCL cases. Of these, only 24 were EBV related miRNAs, being the other human miRNAs differentially expressed.

Conclusions: A microRNA signature in R-CHOP treated DLBCL patients is associated with the clinical outcome. This signature is being validated in a larger group of paraffin embedded samples.

MicroRNA expression profiling is able to identify a miRNA signature differentially expressed among DLBCL subtypes, providing a useful tool to identify differentiation stage-related miRNAs and its potential gene targets.

A set of human miRNAs is differentially expressed in relationship with EBV infection suggesting that EBV can interact with host transcriptome deregulating miRNAs expression.

\section{LS 61}

\section{PD-1, A FOLLICULAR T-CELL MARKER USEFUL FOR RECOGNIZING NODULAR LYMPHOCYTE- PREDOMINANT HODGKIN LYMPHOMA}

Syong H. Nam-Cha, Giovanna Roncador, Lydia Sanchez-Verde, Santiago Montes-Moreno, Agustín Acevedo, Purificación DomínguezFranco, Miguel A. Piris

The nodularity and presence of T-cell rosettes surrounding the neoplastic cells has been described as a defining feature of nodular lymphocyte-predominant Hodgkin lymphoma (NLPHL). We have explored the potential diagnostic value of a new marker (NAT105) that recognizes the antigen PD1 in a series of 152 cases diagnosed as nodular sclerosis Hodgkin lymphoma (NSHL), mixed cellularity Hodgkin lymphoma (MCHL), lymphocyte-rich classical Hodgkin lymphoma (LRCHL), NLPHL and T cell histiocyte-rich B cell lymphoma (T/HRBCL). All the cases were immunostained with a panel of antibodies against CD10, bcl-6, CXCL13, CD57, and PD-1 (NAT-105). The series includes a set of cases diagnosed as NLPHL with diffuse areas, and a group of borderline cases with features between those of NLPHL and T/HRBCL.

Results show that PD-1 (NAT-105), is an excellent immunomarker not only of follicular T-cell rosettes in NLPHL, but not a unique and defining feature of NLPHL, but also of a subset of LRCHLs. The presence of PD-1-positive (NAT-105) T-cell rosettes seems to be an additional useful feature in the differential diagnosis of NLPHL and T/ HRBCL, which is normally a controversial and difficult task. The standard T/HRBCL cases lack follicular T-cell rosettes, while most of the borderline cases between the two entities have follicular T-cell rosettes, thus suggesting a closer relation with NLPHL.

\section{LS 62}

\section{IN VIVO ACTIVATION OF p53 BY NUTLIN-3A, AN MDM2 ANTAGONIST, INDUCES ANTITUMOR ACTIVITY IN FOLLICULAR LYMPHOMA}

Elias Drakos ${ }^{1 *}$, Ellen J. Schlette ${ }^{1}$, George Z. Rassidakis ${ }^{1,2}$, Jiang Li ${ }^{1}$, Richard J. Ford ${ }^{1}$, and L. Jeffrey Medeiros ${ }^{1}$.

Department of Hematopathology The University of Texas MD Anderson Cancer Center, Houston, Texas ${ }^{1}$ and ${ }^{2}$ First Department of Pathology, National and Kapodistrian University of Athens, Athens, Greece.

Background: $\mathrm{t}(14 ; 18)(\mathrm{q} 32 ; \mathrm{q} 21)$, resulting in overexpression of the antiapoptotic protein BCL2, is a hallmark of follicular lymphoma (FL). p53, the most frequently mutated tumor suppressor gene in human cancers, is rarely mutated in FL. In preliminary in vitro studies we showed that activation of the p53 pathway by nutlin-3a induces cell cycle arrest and apoptosis in B-cell lymphoma cell lines with $t$ $(14 ; 18)$ and unmutated p53.

Purpose: We used the B cell lymphoma cell line DOHH2, that has $\mathrm{t}$ $(14 ; 18)$ and wt-p53, and a mouse xenograft model to investigate the in vivo therapeutic potential of nutlin-3a in FL.

Results: In mice with established FL tumors, two weeks of treatment with nutlin-3a reduced tumor volume; mean tumor volume was $1965.5 \mathrm{~mm}^{3}$ in the control group and $107.5 \mathrm{~mm}^{3}$ in the nutlin-3a treated group $(\mathrm{p}<0.02$ Mann Whitney test). Two of the nutlin-3atreated mice had no palpable tumors at the end of treatment. Examination of 6 tumors after 3 injections by Western blotting and immunohistochemistry showed upregulation of p 53 specifically in the nutlin-3a-treated group. In the control versus nutlin-3a-treated groups, the proliferation index (Ki-67) was $92.8 \%$ versus $75.4 \%$, p21-positive tumor cells were $13.8 \%$ versus $42.3 \%$, the apoptotic index (TUNEL) was $3.1 \%$ versus $16.6 \%$, and activated caspase-3-positive tumor cells were $2.1 \%$ versus $15.7 \%$, respectively ( $p<0,05$ Mann Whitney test). Conclusions: p53 reactivation by the MDM2 inhibitor, nutlin-3a, inhibits cell proliferation, induces apoptosis and strikingly inhibits in vivo growth of FL tumors suggesting a new therapeutic approach for patients with FL.

\section{LS 63}

HIGH NUMBERS OF PROGRAMMED-DEATH-1(PD-1)+ CELLS ARE ASSOCIATED WITH IMPROVED OVERALL SURVIVAL (OS) IN GERMINAL CENTER B CELL(GCB) DIFFUSE LARGE B CELL LYMPHOMA(DLBCL)

\footnotetext{
*Pedro Farinha ${ }^{1}$, Giovanna Roncador ${ }^{2}$, Laurie $\mathrm{Sehn}^{3}$, Joseph M Connors $^{3}$ and Randy D Gascoyne ${ }^{1}$.

${ }^{1}$ Pathology and ${ }^{3}$ Medical Oncology, BC Cancer Agency, Vancouver, Canada, and ${ }^{2}$ Monoclonal Antibodies Unit, Biotechnology Program, Spanish National Cancer Centre (CNIO), Madrid, Spain.
}

Background: Cell-of-origin (COO) distinction characterized by geneexpression-profiling (GEP) and validated at protein level provides a biologically relevant DLBCL subclassification. Addition of Rituximab (R) to CHOP chemotherapy improved the outcome in DLBCL, 
due primarily to its effect on the non-germinal center B-cell (GCB) subgroup. The underlying mechanism responsible for this selective impact is unknown. Programmed death-1 $(P D-1)$ belongs to the immunoglobulin $\mathrm{CD} 28$ receptor family and is characteristically expressed by germinal center-associated $\mathrm{T}$ cells.

Aims: Assess the clinical impact of PD-1+ cells in DLBCL, distinguished by $\mathrm{COO}$ subtypes and treated in consecutive eras of therapy with R-CHOP vs CHOP. 163 patients with de novo DLBCL treated between 1999 and 2002 had available paraffin-embeddedformalin-fixed blocks. TMAs were stained with PD-1 (NAT105), CD10, Bcl-6, and MUM1.

Results: Patients were treated with CHOP (81) or R-CHOP (82). The IPI was predictive of OS $(p<0.0001)$. Sixteen cases had uninterpretable immunostains resulting in $71 \mathrm{GCB}$ and 74 non-GCB cases $(n=145)$. Overall, $22 \%$ (32) had $>100$ PD- $1+$ cells, including $20 \mathrm{GCB}$ and 12 non-GCB $\left(\chi^{2}=0.1\right)$. PD-1+>100 predicted a better OS, independently of IPI, in the GCB group ( $p=0.007)$ but not in the non-GCB group $(p=$ $0.61)$. Based on treatment era, PD- $1+>100$ were significantly associated with improved OS in the CHOP group $(\mathrm{p}=0.04)$, and showed a strong trend in the R-CHOP GCB DLBCL cases $(\mathrm{p}=0.1)$.

Conclusion: PD-1+Tcell content predicts OS in DLBCL, due largely to its impact in GCB, suggesting an anti-tumoral immune response present in this subtype. Although benefits are more striking in the pre$\mathrm{R}$ era, there is a clear trend in the post-R GCB group. Additional studies are needed, but these preliminary data provide evidence that non-neoplastic cells affect survival in patients with DLBCL.

\section{LS 64}

\section{THE TUMOR SUPPRESSOR GENE "HYPERMETHYLATED IN CANCER 1 (HIC1)" IS SUPPRESSED IN DIFFUSE LARGE B-CELL LYMPHOMA (DLBCL) BUT IS NOT ASSOCIATED WITH OVEREXPRESSION OF THE SIRT1 DEACETYLASE}

Heike Stöcklein ${ }^{1,2 *}$, Jana Smardova ${ }^{3}$, Jiri Macak $^{3}$, Tiemo Katzenberger ${ }^{1}$, Sylvia Höller ${ }^{1}$, Swen Wessendorf ${ }^{4}$, Grit Hutter ${ }^{5}$, Martin Dreyling $^{5}$, Eugenia Haralambieva ${ }^{1}$, Uwe Mäder ${ }^{6}$, Hans Konrad MüllerHermelink $^{1}$, Andreas Rosenwald ${ }^{1}$, German Ott ${ }^{1,7}$ and Jörg Kalla ${ }^{1}$

${ }^{1}$ Institute of Pathology, University of Würzburg, Germany

${ }^{2}$ Dr Margarete Fischer-Bosch Institute of Clinical Pharmacology, Stuttgart, Germany

${ }^{3}$ Department of Pathology, Medical Faculty of Masaryk University and Faculty Hospital Brno, Brno, Czech Republic

${ }^{4}$ Klinik für Innere Medizin III, University of Ulm, Germany

${ }^{5}$ Medizinische Klinik und Poliklinik III, University of MünchenGrosshadern, Germany

${ }^{6}$ Interdisciplinary Cancer Center, University of Würzburg, Germany

${ }^{7}$ Department of Clinical Pathology, Robert-Bosch-Krankenhaus, Stuttgart, Germany

Chromosomal deletions of $17 \mathrm{p}$, frequently targeting TP53, occur in $\sim 20 \%$ of DLBCL and are thought to be important for tumor pathogenesis. The overlap between TP53 deletions and mutations is poorly understood in DLBCL. Given that TP53 gene deletions are almost invariably associated with loss of the whole chromosomal arm $17 \mathrm{p}$, it remains unclear whether loss of TP53 locus represents the most important biological consequence of $17 \mathrm{p}$ deletions.
A biallelic inactivation of TP53 was detected in 13/55 DLBCL, while 22/55 cases harbour a monoallelic TP53-alteration. A minimally deleted region in $17 \mathrm{p} 13.3$ was defined in 11/55 cases, including the tumor suppressor HICl. Co-deletion of HICl and TP53 was found in 28/55 DLBCL. Hypermethylation of the HIC1-promoter was demonstrated in $30 / 55$ DLBCL, and was associated with monoallelic deletion of the HICl locus in 27/30 DLBCL. Complete inactivation of $\mathrm{HICl}$ $\left(\mathrm{HICl}^{-/}\right)$resulted in significant reduction of $\mathrm{HICl}$ mRNA expression when compared to wildtype HICl DLBCL $\left(\mathrm{HICl}^{+/+}\right)(\mathrm{p}=0.033)$ and was correlated with worse clinical outcome of DLBCL patients.

Since inactivation of $\mathrm{HICl}$ has been reported to result in an overexpression of $S I R T 1$, leading to inactivation of $\mathrm{p} 53$ protein, we investigated SIRT1 mRNA and protein expression. No significant differences in SIRT1 mRNA expression levels were detected comparing DLBCL cases with $\mathrm{HIC}^{+/+}$and $\mathrm{HICl}^{-/-}$status $(\mathrm{p}=0.886)$. Performing immunohistochemistry of SIRT1 protein in 23/55 DLBCL no correlation was shown between SIRT1 mRNA and protein concentration $(\mathrm{p}=0.188)$. Interestingly, ABC-DLBCL presented with decreased SIRT1 protein level compared to GCB-DLBCL $(\mathrm{p}=0.033)$. These findings strongly suggest $\mathrm{HICl}$ as novel, clinical relevant tumor suppressor candidate in DLBCL. SIRT1 mRNA expression is independent of $\mathrm{HICl}$ inactivation status, but is probably regulated on post-transcriptional level in DLBCL. A significant difference of SIRT1 protein expression was detected in ABC and GCB-DLBCL, suggesting that SIRT1 may be a useful tool distinguishing DLBCL of ABC- or GCB-subtype.

\section{LS 65}

\section{CONCURRENT HODGKIN'S LYMPHOMA AND METASTATIC BREAST CARCINOMA IN CERVICAL LYMPH NODES}

*Parasi-Ismailou $\mathrm{A}^{1}$, Lydataki $\mathrm{E}^{2}$, Pantoula $\mathrm{P}^{1}$, Hager $\mathrm{I}^{2}$, Karakosta $\mathrm{A}^{1}$, Tamvakos $\mathrm{H}^{2}$ Laoutaris $\mathrm{N}^{3}$

Pathology ${ }^{1}$, Internal Medicine ${ }^{2}$ and Hematology ${ }^{3}$ Department General Hospital of Nikaia-Piraeus « Agios Panteleimon» Greece

Increased breast cancer risk following treatment for Hodgkin's lymphoma is well established, but the opposite is unknown, although patients with adjuvant radiotherapy and chemotherapy for breast carcinoma are at increased risk of developing second malignancies.

The coexistence of these two particular malignant tumors in a lymph node has, to our knowledge, described only once before.

We describe a case of a 75-year old woman with metastatic ductal carcinoma of the breast and Hodgkin's lymphoma in left cervical lymph nodes, presenting as a collision tumor.

The patient presented with weight loss, fever, anemia and slightly enlarged cervical, subraclavicular, mediastinal and peritoneal lymph nodes. She had undergone a left mastectomy with axillary dissection for breast cancer 9 years before. Because of axillary metastasis, she was treated with adjuvant chemotherapy and hormonal therapy and was well under surveillance until one year ago.

Histologically, one of the lymph nodes showed mixed cellularity classical Hodgkin's lymphoma, while two others showed both metastatic carcinoma and Hodgkin's lymphoma. Immunohistochemistry was typical, highlighting the two populations. 
The pathogenetic mechanisms for the development of lymphoma after therapy for breast cancer, as well as the problems concerning the appropriate staging and therapy of both diseases are discussed.

\section{LS 66}

\section{HISTONE DEACETYLASE 1, 2, 6 AND ACETYLATED HISTONE H4 IN B- AND T-CELL LYMPHOMAS}

*Lena Marquard ${ }^{1,5}$, Christian Bjørn Poulsen ${ }^{1,2}$, Lise Mette Gjerdrum ${ }^{1}$, Peter de Nully Brown ${ }^{2}$, Ib Jarle Christensen ${ }^{3}$, Peter Buhl Jensen ${ }^{4,5}$, Maxwell Sehested ${ }^{1,5}$, Elisabeth Ralfkiær ${ }^{1}$

${ }^{1}$ Experimental Pathology Unit, Department of Pathology, Copenhagen University Hospital, Denmark. ${ }^{2}$ Department of Haematology, Copenhagen University Hospital, Denmark. ${ }^{3}$ Finsen laboratory, Copenhagen Biocenter, Copenhagen University Hospital, Denmark. ${ }^{4}$ Department of Oncology, Copenhagen University Hospital, Denmark. ${ }^{5}$ Topotarget A/S, Copenhagen, Denmark

Histone deacetylase (HDAC) inhibitors are novel therapeutics in the treatment of peripheral T-cell lymphoma, unspecified (PTCLU), and diffuse large B-cell lymphoma (DLBCL), where, for unknown reasons, T-cell malignancies appear to be clinically more responsive than B-cell malignancies. However, the expression of HDACs in DLBCL and PTCLU has not previously been investigated. Thus, the aim was to investigate by immunohistology the expression of HDAC1, HDAC2, HDAC6 and acetylated histone $\mathrm{H} 4$ in pretreatment, diagnostic samples from 31 DLBCL and 45 PTCLU, and to correlate these data with the overall survival. The results showed moderate to high expression of HDAC1, HDAC2, HDAC6, and acetylated $\mathrm{H} 4$ in $80.6 \%, 90.4 \%, 71.0 \%$, and $74.2 \%$ of the DLBCL and in $100 \%, 86.6 \%, 66.6 \%$, and $35.6 \%$ of the PTCLU. By contrast, normal lymphoid tissue showed generally weak expression. HDAC2 was expressed more abundantly than HDAC6 in both DLBCL $(p=0.0006)$ and PTCLU $(p<0.0001)$. Also, HDAC1 was more abundantly expressed than HDAC6 in PTCLU $(p<0.0001)$. HDAC1 was more abundantly expressed in PTCLU than in DLBCL $(p=0.0046)$, whereas acetylated $\mathrm{H} 4$ was more frequent in DLBCL $(\mathrm{p}<0.0001)$, the latter intriguingly suggesting a mechanism for the higher sensitivity of T-cell lymphomas to HDAC inhibitors. No differences in expression were detected between GCB vs. non-GCB DLBCL. Regarding survival, moderate to strong HDAC6 expression was significantly correlated with favourable outcome $(p=0.016)$ for DLBCL patients compared to low expression, whereas the opposite effect was observed for PTCLU patients $(p<0.0001)$. Expression of HDAC1, HDAC2, and acetylated $\mathrm{H} 4$ did not correlate with survival $(p>0.05)$. In conclusion, it is shown that HDAC1, HDAC2, HDAC6, and acetylated H4 are often overexpressed in DLBCL and PTCLU compared to normal lymphoid tissue. Furthermore, it is suggested that HDAC6 may be an important beneficial prognostic marker in DLBCL patients, whereas the opposite effect is observed in PTCLU.
LS 67

\section{LOW RATIO OF INTERFOLLICULAR CD8/FOXP3- POSITIVE REGULATORY T CELLS ASSOCIATED WITH FAVORABLE OVERALL SURVIVAL IN FOLLICULAIR LYMPHOMA}

Magda SAIFI ${ }^{1,2}$, Thérèse ROUSSET ${ }^{1}$, Mounia BRAZA ${ }^{2}$, Jean François $\operatorname{ROSSI}^{2,3}$ and Valérie COSTES ${ }^{* 1,2}$ :

Pathology ${ }^{1}$ and Hematology ${ }^{2}$ departments, INSERM U847, CHU Montpellier, 80 Av A Fliche 34295 Montpellier cedex5, France

Several studies have highlighted the importance of the microenvironnement in the behaviour of follicular lymphoma. Immunochemical analyses were conducted to study CD8, CD68 an forkhead box protein P3 (FOXP3) positive $\mathrm{T}$ regulatory cell numbers and distributions in 84 patients with follicular lymphoma (58 at diagnosis, 26 at relapse). Since the overall effect of Tregs is considered to depend on their number in relation to $\mathrm{CD} 8+$ effectors $\mathrm{T}$ cells, we determined the corresponding ratio for each patient and correlated the results with clinical parameters.

The interfollicular CD8/FOXP3+ cell ratio was significantly higher in patients with grade 3 tumors (2.04 versus 1.63) and with a high risk FLIPI index (2.99 versus 1.53) compared with those with grade 1-2 tumors or a low-intermediate FLIPI index. The same results were obtained with the intrafollicular CD8+/FOXP3 + cell ratio. The interfollicular CD8/FOXP3 ratio was found to have prognostic value for overall survival with a 5 -years OS of $82 \%$ versus $59 \%$ for a ratio of less or more than 1.68. Moreover, an interfollicular FOXP3+ cell number of more than 86 cells $/ \mathrm{mm}^{2}$ was correlated with a more favourable outcome $(p=0.03)$.

When comparing the two groups at diagnosis and relapse, we observed a significant difference between the number of CD68 cells both in intrafollicular locations (39.23 at diagnosis versus 32 at relapse, $\mathrm{p}=0.07$ ) and interfollicular locations (96 at diagnosis versus 62 at relapse, $\mathrm{p}=0.05$ ). The $\mathrm{CD} 8 / \mathrm{FOXP} 3$ ratio in interfollicular locations was significantly different (1.66 at diagnosis versus 2.2 at relapse, $\mathrm{p}=0.05$ ).

The presence of abundant FOXP3+ cells with a low CD8/FOXP3 ratio is probably the mark of an active immune response during tumor development, with lymphoma cells acting as targets or bystanders.

\section{LS 68}

\section{DIFFERENCES IN GENE EXPRESSION PATTERNS BETWEEN HODGKIN LYMPHOMA SUBTYPES ARE SHAPED BY INFILTRATING MACROPHAGES}

Birgersdotter A. ${ }^{1}$, Baumforth K.R.N. ${ }^{2}$, Sjöberg J. ${ }^{3}$, Murray P.G. ${ }^{2}$, Ernberg I. ${ }^{1}$, Björkholm M. ${ }^{3}$ Porwit A. ${ }^{4}$

1) Department of Microbiology, Tumorbiology and Cell biology, MTC, Karolinska Institute, Sweden

2) C.R.U.K. Institute for Cancer Studies, University of Birmingham, Birmingham, UK

3) Department of Medicine, Division of Haematology, Karolinska University Hospital, Stockholm, Sweden

4) Department of Pathology, Karolinska University Hospital, Stockholm, Sweden 
Background: Nodular Sclerosis (NS) and Mixed cellularity (MC) constitute most of classical Hodgkin Lymphoma (HL) cases. Hodgkin/ Reed-Sternberg (H-RS) cells in both subtypes have similar phenotype but lymph node morphology differs. There are also differences in epidemiological features and clinical characteristics of patients.

Purpose of study: To define at molecular level differences between NS and MC HL subtypes and to identify involved cell types.

Methods: The Affymetrix platform was used to determine gene expression profiles in whole tissue samples of $44 \mathrm{HL}$ (25 NS and 19 MC). Results were validated by RT-PCR and on protein level by immunohistochemistry. Data was analyzed in relation to morphological features of tumors and clinical characteristics of the patients.

Results: 265 genes had higher expression in the NS subtype and 20 in the MC subtype. Microarray analysis clearly distinguished genes related to NS specific fibrosis. NS samples showed extra cellular matrix (ECM) deposits similar to wound healing (COL1, COL3, $L U M)$ and high expression of genes involved in ECM remodeling. Immunohistochemistry results showed that mainly macrophages and fibroblasts expressed these proteins. Genes with higher expression in the MC tumors were inflammation-related: $C 1 Q \alpha, C 1 Q \beta, C X C L 9$. The C1Q subunits were mainly expressed by infiltrating macrophages and levels of expression correlated also with age of the patients.

Conclusion: Gene expression and morphology of NS and MC HL subtypes are linked with regard to tissue fibrosis and cell composition of tumors. NS has features of a second phase wound-healing process while MC samples lack these deposits of ECM. Both subtypes show variable expression of different inflammation-related genes correlating to the numbers of macrophages. Our work underlines the role that macrophages might have in shaping the gene expression to whole tumor samples.

\section{LS 69}

\section{TRANSFORMATION OF B-CELL LYMPHOMA TO HISTIOCYTIC SARCOMA - REPORT OF TWO CASES}

Assia Bassarova ${ }^{1}$, Gunhild Trøen ${ }^{1}$, Alexander Fosså ${ }^{2}$, Ida Munster Ikonomou $^{1}$, Jahn M. Nesland ${ }^{1}$, Jan Delabie ${ }^{1}$

${ }^{1}$ Pathology Clinic, The National Hospital - The Norwegian Radium Hospital, Oslo, Norway

${ }^{2}$ Department of Oncology, The National Hospital - The Norwegian Radium Hospital, Oslo, Norway

Tumors of histiocytes and dendritic cells are extremely rare and represent less then $1 \%$ of all the tumors in the lymph nodes. Some of those tumors occur in patients with another malignant tumor. For example, histiocytic sarcoma occurs in patients with previously diagnosed mediastinal germ cell tumors, other cases may be associated with malignant lymphomas. To our knowledge, no histiocytic sarcomas have been described occurring as transformed tumors from B-cell lymphomas.

We report two patients who initially presented with B-cell lymphomas and developed later in the course of the disease tumors with morphological and immunohistochemical characteristics of histiocytic sarcoma. The first patient was a 62 year old man, presenting with diffuse large B-cell lymphoma on the tongue. One year later, a second biopsy from the tongue showed a recurrence with large pleomorphic cells. A similar lesion subsequently occurred on the abdomen. Immunohistochemical results showed lack of all B-cell markers, including PAX-5, but strong positivity for S-100, PU-1, CD68 (PGM1) with focal positivity for CD1a instead. Clonal rearrangement studies performed on both biopsies from the tongue and the skin showed the same $\operatorname{IgH}$ chain rearrangement. The second patient was 53 year old man with follicular lymphoma. Eight years after the first presentation grade 3B follicular lymphoma was diagnosed. Thirteen years after the initial diagnosis the patient developed a quickly growing tumor on the neck and this time the biopsy showed a diffuse infiltration with pleomorphic tumor which lacked all B-cell markers, including PAX-5, but expressed S-100, PU-1, CD14, CD68 (PG-M1), MAF-B and focal positivity for CD1a. Clonal rearrangement studies, together with cytogenetic data performed on one of the lymph node biopsies and the tumor on the neck, showed the same $\operatorname{IgH}$ chain rearrangement and karytoype with $\mathrm{t}(14 ; 18)$.

These two cases illustrate a rare form of transformation of B-cell neoplasia to histiocytic sarcoma. The clonal relationship is proven by immunoglobulin gene rearrangement studies. These cases demonstrate an unusual plasticity of mature neoplastic B-cells with downregulation of B-cell markers and B-cell transcription factors and upregulation of histiocytic markers and transcription factors.

\section{$\underline{\operatorname{LS} 70}$}

\section{CHLAMYDIA INFECTION AND THE DEVELOPMENT OF THE OCULAR ADNEXAL LYMPHOMAS}

Alessandro Carugi ${ }^{1 *}$, Anna Onnis ${ }^{1}$, Anna Luzzi ${ }^{1}$, Gian Marco Tosi ${ }^{2}$, Cristiana Bellan ${ }^{1}$, Mario Cocco', Giulia De Falco ${ }^{1}$, Antonio Giordano $^{1,3}$ and Lorenzo Leoncini ${ }^{1}$

${ }^{1}$ Dpt. Human Pathology and Oncology, University of Siena, Italy

${ }^{2}$ Dpt. of Ophtalmology, University of Siena, Italy

${ }^{3}$ Sbarro institute for Cancer Research \& Molecular Medicine, Temple University, Philadelphia PA 19122, USA

Non-Hodgkins lymphomas develop from nodal and extra-nodal tissue. A particular extra-nodal lymphoma type arises from B cells of the marginal zone (MZ) of mucosa-associated lymphoid tissue (MALT). The geographic heterogeneity in the incidence of B-cell non Hodgkin's lymphomas and the growing evidence suggest that MZ lymphomas are associated with chronic antigenic stimulation by microbial pathogens, among which $H$. pylori-associated gastric MALT lymphoma is the best studied.

Recently, MALT lymphomas have been described in the context of chronic conjunctivitis, which can be associated with Chlamydia infection. Studies from Italy showed Chlamydia psittaci in $87 \%$ of ocular adnexal MALT lymphomas and complete or partial regression of the lymphoma after $C$. psittaci eradication in four of nine cases. However $C$. psittaci was not founded in ocular adnexal lymphoma from other studies. As association with $C$. psittaci does not seem to be a constant parameter, this variability may depend on geographic heterogeneity.

This project was designed to further investigate the role of Chlamydia psittaci in the development of ocular adnexal MALT lymphomas, by comparison of cases retrieved from different geographic areas, as Kenia and Italy.

The presence of $C$. psittaci DNA in biopsies of ocular adnexal lymphomas was demonstrated by TETR-PCR, a modified PCR reaction, used to amplify different DNA sequences in the variable 
regions of the 16S and 23S spacer rRNA genes specific for Chlamydia psittaci.

DNA was extracted from 31 ocular adnexal lymphomas, retrieved from two different geographical regions. The prevalence of C. psittaci infection in MALT lymphoma showed a marked variation between the two geographical regions. $20 \%(5 / 25)$ of the samples from Italy were positive for $C$. psittaci, but no association with this pathogen was observed in any of the samples from Kenya.

Furthermore, we investigated a possible relationship between $C$. psittaci infection and the promoter hypermethylation of p16/INK4a. This epigenetic alteration has been described in H. pylori-associated gastric MALT lymphoma. Our results show a partial methylation of p16/INK4a promoter in $46 \%(12 / 26)$ of $C$. psittaci-negative cases, whereas no hypermethylation of this gene was found in C. psittacipositive cases. As genetic alterations, as the $\mathrm{t}(11 ; 18)$, have been described in $H$. pylori-associated MALT lymphomas, we are currently performing FISH studies to evaluate whether such genetic alterations may also occur in adnexal lymphomas, besides Chlamydia association. From these findings, we may conclude that many and different infectious agents may play a critical role in MALT lymphoma development.

\section{LS 71}

\section{EUPHORBIA TIRUCALLI AS A POSSIBLE COFACTOR IN INDUCING EBV-DRIVEN MALIGNANT TRANSFORMATION}

Mario Cocco ${ }^{1 *}$, Susanna Mannucci ${ }^{1}$, Giuseppina Antonicelli ${ }^{1}$, Alessandro Carugi ${ }^{1}$, Anna Luzzi ${ }^{1}$, Giulia De Falco ${ }^{1}$, Lorenzo Leoncini ${ }^{1}$ and Cornelia A. van den Bosch ${ }^{2}$

1- Dept. Human Pathology and Oncology, University of Siena, Italy 2- Health Protection Agency, UK

Endemic Burkitt lymphoma (eBL) is a monoclonal B-lymphocytic malignancy and is the most common childhood cancer in subSaharian Africa. The infection with the Epstein-Barr virus (EBV) and the occurrence of holoendemic malaria are two clearly defined agents that are associated with the development of eBL. However, it has been recently postulated a possible role for Euphorbia tirucalli, a plant commonly used in the traditional medicine, as a cofactor in the development of eBL. Latex of this plant is usually used as glue and is played by children in endemic areas of occurrence of BL. Recent findings suggest that latex of this plant may induce EBV lytic cycle in latently EBV-infected cells.

The aim of our study was to investigate the molecular mechanisms, which may be involved in malignant transformation induced by $E$. tirucalli. We treated EBV-infected non-transformed B lymphoblastoid cell lines with different concentrations of plant extracts of $E$. tirucalli and monitored cell proliferation. Our results indicate that there was no difference in cell proliferation upon E. tirucalli treatment. Interestingly, cells treated with E. tirucalli showed a modulation of LMP-1 and EBNA2 expression and a marked up-regulation of Bcl-2.

In order to investigate whether treatment with E. tirucalli was able to induce chromosomal alterations, we performed FISH analysis, using probes designed to detect $c-M Y C$ translocation.
Collectively, our preliminary data suggest that E. tirucalli may cooperate in inducing malignant transformation, due to its modulation of the expression of the latency genes of EBV, and the up-regulation of an anti-apoptotic factor as Bcl-2.

\section{LS 72}

\section{ICOS IS EXPRESSED IN FOLLICULAR T HELPER CELLS AND IN ANGIOIMMUNOBLASTIC T CELL LYMPHOMA}

Teresa Marafioti ${ }^{1}$, Erica Ballabio ${ }^{1}$, Jennifer Paterson ${ }^{1}$, Andreas $\mathrm{Chott}^{2}$, Stefano Pileri ${ }^{3}$, Martin L Hansmann ${ }^{4}$, Harald Stein ${ }^{5}$, David Y Mason ${ }^{1},{ }^{*}$ Philippe Gaulard ${ }^{6}$

${ }^{1}$ Leukaemia Research Fund Immunodiagnostics Unit, Nuffield Department of Clinical Laboratory Sciences, John Radcliffe Hospital, Oxford, UK; ${ }^{2}$ Department of Pathology, University of Vienna, Austria; ${ }^{3}$ Chair of Pathology and Unit of Haematopathology, "L\&A Seragnoli" Institute of Haematology, University of Bologna, Italy; ${ }^{4}$ Institute of Biochemistry II, Goethe University School of Medicine, University Clinic Frankfurt am Main, Germany; ${ }^{5}$ Institute of Pathology, Campus Benjamin Franklin, Charité University Medicine Berlin, Germany; ${ }^{6}$ Département de Pathologie \& Inserm U841, Hôpital Henri Mondor, Créteil, France

Follicular T-helper cells $\left(\mathrm{T}_{\mathrm{FH}}\right)$ represent a population of $\mathrm{T}$ cells residing in germinal centres of lymphoid follicles. These cells show a unique gene expression profile and are believed to be the cell of origin of angioimmunoblastic $\mathrm{T}$ cell lymphoma (AITL). We have previously described new proteins, SAP, PD-1 and CXCL13, that labelled $\mathrm{T}_{\mathrm{FH}}$ (Roncador et al., 2007; Dupuis et al, 2006) and the majority of AITL cases analysed.

We report here an antibody that recognises the Inducible COStimulator protein (ICOS), a member of the CD28 family that plays a critical role in T cell-dependent humoral immunity and is expressed in NK cells, subsets of regulatory $\mathrm{T}$ helper and (especially) in $\mathrm{T}_{\mathrm{FH}}$ cells.

ICOS antibody was studied in normal and neoplastic paraffinembedded lymphoid tissues by immunohistochemistry. In tonsil and lymph node, ICOS-positive cells were found in the inner part of the mantle at the periphery of the light zone (suggestive of $\mathrm{T}_{\mathrm{FH}}$ cells) and some in the interfollicular area. In the thymic medulla, medium-sized cells expressed ICOS and only rare scattered ICOS-positive cells were found in spleen and bone marrow. Double immunolabelling revealed that ICOS is expressed on $\mathrm{T}_{\mathrm{FH}}$ cells (as demonstrated by its coexpression with SAP, PD-1, CD57 and BCL-6), and on a subset of FOXP3-positive regulatory T-cells.

The analysis of ICOS in 386 neoplasms (comprising almost all types of B-, T-cell and Hodgkin lymphomas) revealed that B-cell lymphomas were negative in all instances and among T-cell lymphomas ICOS expression was restricted to 27/29 of AITL cases and 14/29 of peripheral T-cell lymphoma (PTCL)-unspecified. The presence of ICOS-positive PTCLs is an interesting finding, it might indicate the activation status of neoplastic cells or suggest the existence of a biologically distinct group of "AITL-like PTCLs" as described by recent gene expression profiling studies (de Leval et al., 2006). 


\section{$\underline{\text { LS } 73}$}

\section{THREE DISTINCT PROTEOMIC SIGNATURES FOR CHRONIC LYMPHOCYTIC LEUKEMIA LYMPH NODES}

Corine Jansen ${ }^{1}$, Martijn de Prenter ${ }^{1}$, Konnie Hebeda ${ }^{1}$ MD, PhD, Marian Stevens-Kroef ${ }^{2} \mathrm{PhD}$, Annet Simons ${ }^{2} \mathrm{PhD}$, Reinier Raymakers ${ }^{3}, \mathrm{MD}, \mathrm{PhD}$, Johan van Krieken ${ }^{1} \mathrm{MD}, \mathrm{PhD}$, and Patricia JTA Groenen ${ }^{1} \mathrm{PhD}^{*}$

Dept. of Pathology, Dept of Human Genetics, dept of Hematology, Radboud University Nijmegen Medical Centre, Nijmegen, The Netherlands.

Background: Lymph nodes from nodal (lymphoma) and leukemic CLL patients were analysed using Surface Enhanced Laser Dissection/ Ionization-Time of Flight Mass Spectrometry (SELDI-TOF MS) to generate specific protein signatures of the lymph node protein homogenates. In addition, the $I G H$-mutation status, $\mathrm{V}_{\mathrm{H}^{-}}$family usage, and the genomic aberrations were determined on the same set of CLL lymph node specimens.

Aim of study: To identify correlations between the distinct protein

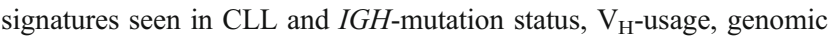
aberrations or nodal/leukemic presentation of CLL.

Results: Using protein profiling by SELDI-TOF MS we have identified three distinct protein signatures, i.e.: $1 \mathrm{~A}, 1 \mathrm{~B}$ and 2 , in 12 , 11 and 12 of the lymph nodes from CLL patients, respectively.

The protein signatures do not correlate with the prognosis of the patients, as defined by the unmutated $I G H$ - status, or by $\mathrm{V}_{\mathrm{H}} 3-21$ usage, which is adverse prognostic factor in CLL independent of the mutation status. Neither, can we find a correlation with the $\mathrm{V}_{\mathrm{H}} 1-69$ family usage, which is the most frequently rearranged $I G H V$ gene in CLL.

Preliminary data provide evidence that protein signature 2 is not found in the lymph nodes of leukemic CLL patients, although the group assessed is very limited. It is expected that the proteomic signatures will associate with specific genomic aberrations in the samples. Data analysis on the occurrence of trisomy 12 and of genomic aberrations at $A T M$ (11q22), DLEU and RB1 (13q14), TP53 (17p13) gene, which will be determined with the multiplex ligation dependent probe amplification technique, are in progress.

In conclusion: Specific proteomic signatures are identified in lymph nodes of leukemic and nodal CLL patients. The distinct proteomic signatures do not correlate with the $I G H$-mutation status, but it is highly likely that a correlation with the genomic aberrations will be found

\section{LS 74}

\section{ASSESSMENT OF THE MICROBIOLOGICAL DIVERSITY IN PULMONAL EXTRANODAL MARGINAL ZONE B-CELL LYMPHOMAS OF MALT-TYPE (BALT-LYMPHOMA) BY 16S RDNA HETEROGENEITY ANALYSIS USING RFLP AND $P$ HYLOGENETICS (SHARP-SCREENING)}

P. Adam ${ }^{1 *}$, U. Hentschel ${ }^{2}$, C. Gernert ${ }^{2}$, S. Schmitt ${ }^{2}$, E. Haralambieva ${ }^{1}$, G. Ott ${ }^{1}$, H.K. Müller-Hermelink ${ }^{1}$

${ }^{1}$ Institut für Pathologie, Universität Würzburg

${ }^{2}$ Zentrum für Infektionsforschung, Universität Würzburg
Background: For different localisations of extranodal marginal zone B-cell lymphoma of MALT type (eMZBCL) an association with chronic inflammation caused by microbiological agents (e.g. Helicobacter pylori in the stomach) is known. In the lung a link between lymphomagenesis and a defined causative organism is still missing. Purpose: A comprehensive diversity survey using 16S-rDNA library construction followed by restriction-fragment-length-polymorphism (RFLP) analysis, sequencing and phylogenetic tree construction was employed on nine cases each of BALT lymphoma and control lung tissues (normal fetal lung, pneumonitis, emphysema, cancer) to assess the respective bacteriological microenvironment.

Results: This highly sensitive method, hereafter termed "SHARPScreening", allowed for the identification of the entire bacterial population in the tissue in a cultivation-independent manner.

Noteworthy, in eight of the nine cases of BALT lymphoma, bacteria of the Alcaligenaceae family (Alcaligenes, Achromobacter, AKIW733), were found, whereas none of the control cases showed the presence of these clades.

Conclusions: 16S-rDNA library construction in combination with RFLP-screening and phylogenetic analyses, hereafter described as "SHARP-Screening" is a cultivation-independent tool for the analysis of the bacteriological microenvironment in chronic inflammation processes, giving rise to extranodal marginal zone B-cell lymphomas of MALT-type. Betaproteobacteria of the Alcaligenaceae family may be affiliated with and possibly involved in the lymphomagenesis of BALT lymphomas.

\section{LS 75}

\section{CURRENT ROUTINE CLONALITY DETECTION DOES NOT DISCRIMINATE BETWEEN HODGKIN LYMPHOMA AND B-CELL NON-HODGKIN LYMPHOMA.}

Konnie M. Hebeda* MD, PhD , Margarethe C. van Altena, Paul Rombout, Johan H.J.M. van Krieken MD, PhD and Patricia J.T.A. Groenen, PhD.

Department of Pathology, Radboud University Nijmegen Medical Centre, Nijmegen, The Netherlands

Background: B-cell clonality detection in whole tissue sections is considered indicative of B-cell non-Hodgkin lymphoma (B-NHL). Although Hodgkin lymphoma (HL) is a B-cell lymphoma, due to sparse tumor cells, heavy somatic hypermutation load and lack of immunoglobulin expression, the clonal B-cell nature is considered difficult to detect. Aim of study: To investigate the possibility to detect clonality in Hodgkin lymphomas by using multiplex PCR-primer sets for $I G H$ and $I G K$ gene rearrangements.

Results: We tested 25 classical HL with a varying tumor cell load with multiplex PCR primer sets for the incomplete (DJ) and complete (VDJ) $I G H$ gene rearrangements as well as the $I G K$-VJ and $I G K-\mathrm{DE}$ gene rearrangements (BIOMED-2).

A clonal population was found in 15 cases with the $I G H$ FR1-3 PCRs. Using the $I G K$-VJ and $I G K$-DE PCRs an additional 5 cases had a dominant clonal cell population, resulting in a detection rate of $80 \%$. This detection was obtained by using frozen tissue. Paraffinembedded tissue samples of the cases with sufficient PCR-amplify ability allowed detection of the same targets.

No correlation was found with the presence of Epstein Barr Virus in the tumor cells. None of the HL cases showed evidence of B-cell lymphoma during follow-up of 1 to 6 years. 
In conclusion: The present routine PCR methods are sensitive enough to detect small numbers of malignant cells in HL, with a similar rearrangement pattern as B-NHL. Therefore the presence of a clonal B-cell population does not differentiate between HL and NHL.

\section{LS 76}

\section{AP-1 DEPENDENT GALECTIN-1 EXPRESSION DELINEATES CLASSICAL HODGKIN AND ANAPLASTIC LARGE CELL LYMPHOMAS FROM OTHER LYMPHOID MALIGNANCIES WITH SHARED MOLECULAR FEATURES}

Scott J. Rodig ${ }^{1 *}$, Jing Ouyang ${ }^{2}$, Przemyslaw Juszczynski ${ }^{2}$, Treeve Currie $^{1}$, Kenneth Law ${ }^{1}$, Donna S. Neuberg ${ }^{2}$, Gabriel A. Rabinovich ${ }^{3}$, Margaret A. Shipp ${ }^{2}$, and Jeffery L. Kutok ${ }^{1}$

${ }^{1}$ Department of Pathology, Brigham and Women's Hospital, and ${ }^{2}$ Department of Medical Oncology, Dana Farber Cancer Institute, Boston, Massachusetts, USA;

${ }^{3}$ Laboratory of Immunopathology. Institute of Biology and Experimental Medicine (IBYME-CONICET), C1428- Buenos Aires, Argentina

Background: Galectin-1 (Gal1) is an immunomodulatory glycanbinding protein regulated by an AP1-dependent enhancer in Hodgkin cell lines. In recent studies, we found that Hodgkin cell derived Gall promotes the development of the immunosuppressive $\mathrm{T}$ helper $2 / \mathrm{T}$ regulatory cell skewed microenvironment that is typical of classical Hodgkin lymphoma (cHL).

Purpose and results: We wished to determine whether AP1dependent Gall expression is a feature of primary $\mathrm{cHL}$ and whether this signature can distinguish cHL from other lymphoid malignancies. We found that Gall is selectively expressed by Reed-Sternberg cells in $>90 \%$ of an extensive series of primary cHLs and that Gall expression is concordant with that of the activated AP1 component, cJun. In contrast, aggressive B-cell lymphomas, including diffuse large B-cell lymphoma, primary mediastinal large B-cell lymphoma, and nodular lymphocyte-predominant Hodgkin lymphoma, do not express Gall. However, an aggressive tumor of T-cell lineage, anaplastic large cell lymphoma (ALCL), consistently expresses both Gall and its transcriptional regulator, c-Jun. The presence of activated c-Jun, indicative of functional AP1 activity, was confirmed by phospho-cJun immunostaining in cHL and ALCL.

Conclusions: These findings establish a functional AP1 signature that includes Gall expression in cHL and ALCL, and suggests a common mechanism for tumor immunotolerance. In addition, Gall and c-Jun serve as diagnostic biomarkers that delineate cHL and ALCL from other lymphomas with shared morphologic and/or molecular features.

\section{$\underline{\text { LS } 77}$}

\section{EXTRANODAL FOLLICULAR DENDRITIC CELL SARCOMA. A REPORT OF THREE NEW CASES}

D Meseure* (1), M Trassard (1), S Boudjemaa (2), JM Guinebretière (1), C Copie (3) si tu es OK

Department of pathology of (1) centre René-Huguenin Saint-Cloud, (2) Hôpital Trousseau Paris, (3) Hôpital Henri Mondor Créteil FRANCE
Follicular dendritic cell sarcoma (FDCS) are among the rarest of tumours affecting the lymphoid tissue. Their nodal location, the most frequent, is well characterized. It is composed of spindle and ovoid cells in a lymphocytes rich background. Sometimes FDCS is associated with other disease (Castelman disease, EBV infection, inflammatory myofibroblastic tumour, follicular lymphoma...). In their extranodal location, only 46 cases FDCS have been reported. It is often misdiagnosed due to numerous distinctive histologic features which can mimic a wide range of other tumours.

The 3 patients, 53 (a), 63 (b) and 73 (c) year-old women, presented with an intra abdominal mass, respectively located in perirectal (b), mesentery (c) and perihepatic region (a). Surgical resections were performed. The tumours which measured from 3 to $12 \mathrm{~cm}$ (a) were well delineated, whitish, fleshy or fasciculate. Histologically, they all associated areas with typical low grade FDCS, diffuse for one, limited and associated with predominant epithelioid and spindle areas () and for one high grade areas (necrosis, $>20$ mitoses/10 HPF). The tumoral cells diffusely expressed the FDC markers CD21 (3/3), CD23 (2/3) and CD35 (2/3) in typical areas, focally for S100 and CD68 while negative for other mesenchymal markers, and EBV (LMP1, EBER). Additional markers, clusterin (3/3), EGFR (1/3), podoplanin (3/3) fascin $(1 / 3)$ were positive in typical and interestingly in atypical areas. One local recurrence occurred 18 months after incomplete surgical resection (a).

In conclusion, extranodal FDCS are rare and misdiagnosed neoplasm, due to the morphologic diverse histologic features. Recently, different new markers have been reported in FDCS and as we found them positive in non morphological evocative area, might be particularly helpful to recognize this aggressive neoplasm.

\section{$\underline{\text { LS } 78}$}

\section{MYC TRANSLOCATION-NEGATIVE CLASSICAL BURKITT LYMPHOMA CASES: AN ALTERNATIVE PATHOGENETIC MECHANISM INVOLVING MIRNA DEREGULATION}

Leucci Eleonora ${ }^{1}$, Cocco Mario ${ }^{1}$, Onnis Anna ${ }^{1}$, van Cleef Patricia ${ }^{2}$, Bellan Cristiana $^{1}$, van Rijk Anke ${ }^{2}$, De Falco Giulia ${ }^{1}$, Nyagol Joshua ${ }^{1}$, Byakika Bessie $^{3}$, Lazzi Stefano ${ }^{1}$, Tosi Piero ${ }^{1}$, van Krieken Han ${ }^{2}$, Leoncini Lorenzo ${ }^{1 *}$

1.Department of Human Pathology and Oncology, University of Siena, Italy

2.Department of Pathology, Radboud University Nijmegen Medical Center, Nijmegen, The Netherlands

3. The Nairobi Hospital, Nairobi, Kenya

The molecular feature of Burkitt lymphoma (BL) is the translocation, that places c-Myc under the control of immunoglobulin gene regulatory elements. However, there is recent evidence that infrequent cases may lack an identifiable $M Y C$ translocation. In addition, during the EUROFISH project, aiming at the standardization of FISH procedures in lymphoma diagnosis, we found that 5 cases out of 35 classic endemic BLs were negative for $M Y C$ translocations by using a split-signal as well as a dual-fusion probe.

In this study we investigated the expression pattern of miRNAs predicted to target c-Myc, in BL cases, to clarify whether alternative pathogenetic mechanisms may be responsible for lymphomagenesis in cases lacking the MYC translocation. MiRNAs are a class of small RNAs that are able to regulate gene expression at the post- 
transcriptional level. Several studies have reported their involvement in cancer and their association with fragile sites in the genome. They have also been shown to control cell growth, differentiation and apoptosis, suggesting that these molecules could act as tumour suppressors or oncogenes.

Our results demonstrated a modulation of specific miRNAs. In particular, a down-regulation of Let7c was observed in BL cases, compared to normal controls. More interestingly, hsa-mir-34b was found to be downregulated only in $\mathrm{BL}$ cases that were negative for $M Y C$ translocation, suggesting that this event might be responsible for c-Myc deregulation in such cases. This hypothesis was further confirmed by our in vitro experiments, which demonstrated that increasing doses of synthetic hsa-mir-34b were able to modulate c-Myc expression. These results indicate for the first time that hsa-mir-34b may influence c-Myc expression in Burkitt lymphoma as the more common aberrant control exercised by the immunoglobulin enhancer locus.

\section{LS 79}

\section{B CELL DIFFERENTIATION IN EBV-POSITIVE BURKITT LYMPHOMA IS IMPAIRED AT THE POST-TRANSCRIPTIONAL LEVEL BY MIRNA ALTERED EXPRESSION}

Leucci $E^{1}$, Onnis $A^{1}$, Cocco $M^{1}$, De Falco $\mathrm{G}^{1}$, Costanzo $\mathrm{V}^{1}$, Cerino $\mathrm{G}^{1}$, Manucci $\mathrm{S}^{1}$, Cantisani $\mathrm{R}^{1}$, Nyagol $\mathrm{J}^{1}$, Mwanda $\mathrm{W}^{2}$, Iriso $\mathrm{R}^{3}$, Schurfeld $\mathrm{K}^{4}$, Bellan $\mathrm{C}^{1}$, Lazzi $\mathrm{S}^{1}$ and Leoncini $\mathrm{L}^{1 *}$.

1. Department of Human Pathology and Oncology, University of Siena, Siena Italy.

2. Department of Pathology, Kenyatta National Hospital, University of Nairobi, Nairobi, Kenya.

3. St. Mary's Hospital, Lacor, Gulu, Uganda.

4. Dpt. of Human Pathology, Montevarchi, Arezzo, Italy.

Endemic, sporadic and HIV-associated Burkitt lymphoma (BL) all have a similar B-cell phenotype and a $M Y C$ translocation, but a variable association with the Epstein-Barr virus (EBV). According to immunoglobulin gene mutation analysis, EBV-negative BLs may originate from early centroblasts, whereas EBV-positive BLs appear to arise from post-germinal center cells. The appearance of a germinal center phenotype in EBV-positive cells might thus derive from a block in $\mathrm{B}$ cell terminal differentiation. The exit from the germinal center involves a highly complex series of events that is regulated at multiple levels. These events require the activation of BLIMP-1, the master regulator of $\mathrm{B}$ cell terminal differentiation, which in turn represses several target genes, including c-MYC and BCL6.

Recent data indicate that only a small number of genes implicated in immune responses, such as BCL6, SMAD7, BLIMP1, NFAT5, EP300, are preferentially targeted by miRNAs, a class of small RNAs $(\sim 22 \mathrm{nt})$ that are capable of post-transcriptionally regulating hundreds of proteincoding genes and are expressed in a tissue-specific manner during development.

We investigated the expression of specific miRNAs predicted to be involved in B cell differentiation. Among the miRNAs studied, we show here that hsa-miR-127 is strongly up-regulated in EBV-positive BL samples, and that this miRNA is implicated in physiological B cell differentiation. We also demonstrate for the first time that this effect is due to the post-transcriptional regulation of BLIMP-1 and XBP-1.
LS 80

COMPARATIVE PROTEOMIC ANALYSIS OF PRIMARY MEDIASTINAL LARGE CELL LYMPHOMA AND HODGKIN LYMPHOMA USING ITRAQ-LABELING AND TANDEM MASS SPECTROMETRY

Kojo S.J. Elenitoba-Johnson*, Damian Fermin, Venketesha Basrur, Kevin P. Conlon, Charles Seiler, Ian Waxman, Mitchell S. Cairo and Megan S. Lim

Department of Pathology, University of Michigan, Ann Arbor, MI, USA and Department of Pediatrics, Columbia University, New York City, NY, USA

Primary mediastinal large cell lymphoma (PMBCL) is a rare subtype of extranodal diffuse large B-cell lymphoma (DLBCL) that is difficult to distinguish from other types of DLBCL and classical Hodgkin lymphoma (cHL). Recent studies reveal similar gene exression profiles in PMBCL and cHL suggesting a common cell of origin. We utilized a differential isotopic strategy to determine the global proteomic differences between a cell line derived from PMBCL (Karpas 1106P), DLBCL (SUDHL-9), and a cHL (L428). Protein was collected from the cell lysates and subjected to labeling by isobaric tags (iTRAQ) for relative quantification and analyzed by reverse-phase liquid chromatography and electrospray ionization tandem mass spectrometry. $200 \mathrm{~g}$ of total cell lysate was analyzed and LC-MS/MS analyses were performed in duplicate. The proteins were identified using X!Tandem. After normalization, quantitative data were subjected to false discovery rate (FDR) calculation to identify differentially expressed proteins through Mixture Modeling. Differentially expressed proteins were scored at a FDR of 0.13 . This approach revealed 66 proteins with differential expression patterns that were discriminative for the 3 different lymphoma cell lines. Several proteins that have been previously reported to be differentially expressed in these lymphomas were identified including Fascin, Galectin-1, Galectin-3, STAT1 and SWAP70. In addition, several previously unreported proteins involved in numerous cellular functions were differentially expressed (cell adhesion, signaling, immunity). We have validated a subset of these by western blot analysis and immunohistochemistry. This study demonstrates the utility of large-scale mass spectrometry-based proteomics for the elucidation of proteins that may serve as potential diagnostic marker panels for the distinction of PMBCL, DLBCL and cHL.

\section{$\underline{\text { LS 81 }}$}

\section{IS THERE AN IMMUNOPHENOTYPE FOR PLASMABLASTIC LYMPHOMA? IMMUNOHISTOCHEMICAL EVALUATION OF THE PHENOTYPE WITH NEW SPECIFIC MARKERS AGAINST XBP1, BLIMP1, GCET1 AND KLHL6.}

*Santiago Montes-Moreno, Ana-Rosa Gonzalez-Medina, Socorro-María Rodriguez-Pinilla, Lorena Maestre, Lydia Sanchez-Verde, Miguel A Piris.

CNIO. Madrid. Spain.

Plasmablastic lymphoma is a clinicopathological variant of DLBCL, and is considered to derive from terminally differentiated B-cells. Differential diagnosis with ABC-DLCBL and plasma cell myeloma represents a common problem because of the lack of a distinctive phenotype. 
Here we show the results of an immunohistochemical study of 38 cases of Plasmablastic Lymphoma (7 EBV -, $31 \mathrm{EBV}+$ ) using antibodies against CD20, PAX5, BCL6, CD10, GCET1, KLHL6, MUM1, PRDM1/Blimp1, XBP1, CD38, CD138 and ISH for EBV-EBER.

We found here that cases with plasmablastic morphology carry on two different immunophenotypes:

- Full plasmablastic phenotype (Blimp1pos, nucXBP1pos, Pax5 -/+) in 20 cases $(53 \%)$. Of these, the majority shows expression of surface CD138 (13 cases).

- A defective plasmablastic phenotype was observed in $18(47 \%)$ cases, divided into:

- (Blimp1pos, nucXBP1neg, Pax5-/+) was seen in 14 cases $(36 \%)$. Of these, only half showed expression of CD138.

○ Of the remaining 4 cases one was Blimp1neg, nucXBP1 pos, Pax5neg and 3 Blimp1neg, XBP1neg, Pax5-/+.

These full an defective plasmablastic immunophenotypes are rare among conventional DLBCL (including ABC subtypes) $(26 \%$ positivity for Blimp1 and less than $5 \%$ for nuclear XBP1 in a large series of 113 DLBCL)

Interestingly, while Bcl6 was absent in all cases, CD10 and two novel GC markers, GCET1 and KLHL6 were present in a fraction of samples ( 9 cases positive for GCET1 (24\%), 5 cases for KLHL6 $(13 \%)$ and 7 for CD10 (18\%). MUM1 was positive in all cases.

Differences in expression among EBV pos and negative cases were only significant respective to expression of B cell markers CD20 and Pax $5.57 \%$ of EBVneg cases were CD20pos and 42\% PAX5pos while only $13 \%$ and $16 \%$ of EBV positive cases were positive for CD20 and Pax 5 respectively. Our results demonstrate a highly characteristic phenotype of Plasmablastic Lymphoma with the simultaneous double expression of Blimp1 and nuclearXBP1. This particular phenotype helps to differentiate these tumours from DLBCL, independently of EBV status. Moreover, the expression of novel GC markers among these cases facilitates the differential with anaplastic myeloma.

\section{LS 82}

\section{INTRATUMORAL PLASMACYTOID DENDRITIC CELLS AND T CELLS ASSOCIATE WITH INCREASED SURVIVAL IN PATIENTS WITH FOLLICULAR LYMPHOMA (FL)}

\author{
R Butsch ${ }^{1}$, S Schaerer ${ }^{1}$, D Korol ${ }^{2,1}$, E Levi ${ }^{3,4}$, C Renner $^{1}$, N Probst- \\ Hensch $^{2,1}, \mathrm{H} \mathrm{Moch}^{1}$ and $* \mathrm{M}$ Kurrer ${ }^{5,1}$. \\ ${ }^{1}$ Pathology and Oncology, University Hospital Zurich, Zurich, Switzerland; \\ ${ }^{2}$ Cantonal Cancer Registry, University of Zurich, Zurich, Switzerland; \\ ${ }^{3}$ Pathology, John D. Dingell VAMC, Detroit, Michigan, United States; \\ ${ }^{4}$ Pathology, Wayne State University, Detroit, Michigan, United States and \\ ${ }^{5}$ Pathology, Cantonal Hospital Aarau, Aarau, Switzerland.
}

Background: Gene array studies on FL have associated intratumoral macrophage/dendritic cell and/or T cell signatures with both increased and decreased survival. FL has been reported to depend on T cell help for progression. Interferon-alpha, produced by plasmacytoid dendritic cells (pDC), has been used in the therapy of FL. We wanted to test whether this would translate into prognostic factors detectable by immunohistochemistry.
Design: A TMA was constructed with duplicate cores from 252 archival follicular lymphoma samples biopsied between 1990 and 2004. Immunohistochemistry was performed for CD123+ pDC (clone $3 \mathrm{H} 3$, eBioscience) and CD3+ T cells (clone SP7, Lab Vision) on a Ventana Discovery module and quantified by counting stained cells or by stained area fraction determinations. Quantifications were correlated with survival and clinical data including FLIPI in 131 patients. Result: High numbers of CD3 + cells or CD123+ cells associated with increased survival. Low numbers of $\mathrm{CD} 123+$ cells further correlated with B symptoms and elevated LDH. Numbers of CD123+ cells correlated with $\mathrm{CD} 3+$ cells. In multivariate models, however, both CD123 and CD3 proved to be comparable independent prognostic factors. CD123 was more significant than CD3 in predicting $\mathrm{B}$ symptoms and advanced tumor stage, and CD123 was also more significant than grading, FLIPI or age in predicting survival.

Conclusion: $\mathrm{T}$ cells and $\mathrm{pDC}$ associate with and/or influence the clinical course of patients with follicular lymphoma. Association of $\mathrm{CD} 3+\mathrm{T}$ cells and CD123+ pDC with survival suggests that the preservation of the preexisting interfollicular $\mathrm{T}$ cell compartment associates with prognosis. The findings may also suggest, that FL with numerous $\mathrm{T}$ cells is still dependent on $\mathrm{T}$ cells for growth and may therefore carry a better prognosis. CD123+ pDC may associate with longer survival as they endogenously produce the interferon in situ that otherwise would need to be provided by external sources for therapy.

\section{LS 83}

\section{EPIDEMIOLOGICAL DATA OF LEUKEMIAS AND LYMPHOMAS SUGGEST AND SUPPORT DISTINCT BIOLOGICAL GROUPS}

D Poncini ${ }^{1}$, V Stucki ${ }^{1}$, S Giger ${ }^{1}$, D Korol ${ }^{1,2}$, R Maurer ${ }^{3}$, C Renner ${ }^{1}$, N Probst-Hensch ${ }^{1,2}$, H Moch $^{1}$, E Levi $^{4,5}$ and $*$ M Kurrer ${ }^{1,6}$.

${ }^{1}$ Pathology and Oncology, University Hospital Zurich, Zurich, Switzerland; ${ }^{2}$ Cancer Registry, University of Zurich, Zurich, Switzerland; ${ }^{3}$ Pathology, City Hospital Triemli, Zurich, Switzerland; ${ }^{4}$ Pathology, John D. Dingell VA Medical Center, Detroit, Michigan, United States; ${ }^{5}$ Pathology, Wayne State University, Detroit, Michigan, United States and ${ }^{6}$ Pathology, Cantonal Hospital Aarau, Aarau, Switzerland.

Background: Epidemiological data on leukemias and lymphomas (LL) have - in the past - mainly been used for clinical or differential diagnostic purposes. We gathered and reviewed epidemiological data in order to gain insights into the biology of LL.

Design:We performed a retrospective analysis of 11000 LL registered from 1980 until 2004 in the Cancer Registry of the Canton of Zurich (population 1.1 million) to obtain age dependent incidence curves for each entity, corrected for age distribution of the population and year of incidence Result: LL generally show an exponential increase with age, that is genuine to B-cell lymphoma (BCL), T-cell lymphoma (TCL) and hematopoietic stem cell (HPSC) disease, each. LL show a sex ratio (MF) of about 1.75. LL deviating from this include hairy cell leukemia (flat age curve, MF 4) and HL. Nodular sclerosis (NS) HL shows an age peak at age 25 , mixed cellularity (MC) HL shows an exponential increase with age, HL show a MF closer to 1 . PMBCL peaks at age 33 and has a MF of 0.5. ALL/AML drop during adolescence to later show an exponential increase with age. Both marginal zone lymphoma (MZL) and follicular lymphoma (FL) shows a MF of 0.8. FL, however, shows an exponential increase with age 
only until age 65 followed by a leveling off of the incidence curve. Mantle cell lymphoma shows a MF of 6 in the 65-75 year old but a MF of below 2 in the younger and older.

Conclusion: Cancerogenic events affect B-cells, T-cells and HPSC differently. In HPSC diseases epidemiological curves confirm the biological distinction of juvenile forms from senile forms. In LL the epidemilogical incidence curves suggest closer relationships of NSHL with MBCL, and MCHL with other BCL, respectively. Within BCL MCL, FL/MZL and HCL form epidemiologically distinct subgroups in need for biological explanations

\section{LS 84}

\section{EARLY OR LATE DEFECT IN T CELL DEVELOPMENT DIFFERENTLY AFFECT MATURATION AND ORGANIZATION OF THYMIC EPITHELIAL CELLS : LESSON FROM HUMAN PRIMARY IMMUNODEFICIENCIES}

(*) Poliani P.L. ${ }^{1}$, Ravanini $\mathrm{M}^{1}$, Roifman $\mathrm{CM}^{2}$, Villa $\mathrm{A}^{4}$, Facchetti $\mathrm{F}^{1}$, Notarangelo $\mathrm{LD}^{3}$

${ }^{1}$ Department of Pathology, University of Brescia, Italy; ${ }^{2}$ Hospital for Sick Children, Toronto, Canada; ${ }^{3}$ Division of Immunology, Children's Hospital, Boston, USA; ${ }^{4}$ CNR-ITB, TIGET- HSR Milano Italy

Severe combined immune deficiency (SCID) comprises a heterogeneous group of genetic disorders affecting genes of both early (gamma-common $(\gamma \mathrm{c})$ chain, IL-7R, RAG1/2, ADA) and late (MHC class-II, ZAP70) steps of T cell development.

Although SCID are characterized by a similar clinical phenotype, it is being increasingly recognized that hypomorphic mutations in the same genes may result in a more complex phenotype with immunodysreactivity (e.g. Omenn Syndrome, OS).

Cross-talk between thymocytes and thymic epithelial cells (TECs) in the thymus is essential to preserve thymic architecture and function. We have previously shown that the severe lymphoid defect in OS results in disrupted thymic architecture and impaired expression of AIRE (AutoImmune REgulator), a transcription factor expressed by a subset of medullary TEC, and critically involved in negative selection. We have extended this analysis of thymic epithelial cells maturation in eight patients with different forms of SCID, including both early and late defects in $\mathrm{T}$ cell differentiation.

Compartmentalization and maturation of TEC have been studied by expression of cytokeratin- 8 and 5, UEA-1 ligand, claudin- 4 and AIRE, and intrathymic generation of regulatory $\mathrm{T}$ cells (Treg) by Foxp3 expression.

Our data show that early defects in $\mathrm{T}$ cell development result in profound abnormalities of TEC differentiation, characterized by lack of Claudin-4 and AIRE expression.

Later defects in thymic development allow for cortico-medullary demarcation, and normal claudin-4 and aire expression, although in patients with ZAP70 deficiency the medullary-to-cortex ratio is reduced, possibly reflecting impaired thymocyte signalling.
These data may help to explain why immunodysreactivity is most often associated with hypomorphic defects in genes that affect early stages of T-cell development, in which severe TEC abnormalities cause impaired negative selection and inability to convert autoreactive $\mathrm{T}$ cells into Tregs.

\section{$\underline{\text { LS } 85}$}

\section{ASSESSMENT OF PRESERVATION OF B CELL FOLLICLES IN CLASSICAL HODGKIN'S LYMPHOMA RESULTS IN CLINICALLY RELEVANT SEPARATION OF LYMPHOCYTE RICH FROM MIXED CELLULARITY HODGKIN'S LYMPHOMA}

\author{
S. Giger ${ }^{1}$, D Korol ${ }^{2,1}$, E Levi ${ }^{3,4}$, C Renner ${ }^{1}$, N Probst-Hensch ${ }^{2,1}, \mathrm{H}$ \\ Moch $^{1}$ and $*$ M Kurrer ${ }^{5,1}$. \\ ${ }^{1}$ Pathology and Oncology, University Hospital Zurich, Zurich, \\ Switzerland; ${ }^{2}$ Cantonal Cancer Registry, University of Zurich, Zurich, \\ Switzerland; ${ }^{3}$ Pathology, John D. Dingell VAMC, Detroit, Michigan, \\ United States; ${ }^{4}$ Pathology, Wayne State University, Detroit, Michigan, \\ United States and ${ }^{5}$ Pathology, Cantonal Hospital Aarau, Aarau, \\ Switzerland.
}

Background: The WHO classification of Hodgkin's lymphoma (HL) distinguishes nodular lymphocyte predominant (LP) HL from classical HL (cHL). cHL is divided into nodular sclerosis (NS), mixed cellularity (MC) and lymphocyte rich cHL (LR). The WHO classification failed to detail criteria for separation of MC from LR. Follicular and interfollicular patterns of cHL have been described to complement the WHO criteria. We hypothesized that preservation of follicular architecture would separate MC from LR.

Design: Histological slides and clinical follow-up were reviewed from 119 patients diagnosed from 1991 to 2007. Preservation of B cell follicles and absence of sclerosis was used to separate MC and NS from LR. Profiles for EBER, CD20, CD30 and CD15 were used to separate LP from LR.

Result: Review of histologies resulted in 65 NS, 19 MC, 20 LR and $15 \mathrm{NP}$ cases. B-symptoms were more frequent in MC compared to LR and LP. Patients died in 8/65 NS, 6/17 MC, 1/15 LR and 3/14 LP cases. Male to female ratio was for NS 33:32, MC 13:6, LR 14:6 and LP 14:1. MC patients were older, higher stages and B-symptoms were more frequent at diagnosis than in NS and LR. EBER positivity was similar all cHL.

Conclusion: Separation of LR from MC and NS can be achieved by the sole absence of sclerosis and preservation of $\mathrm{B}$ cell follicles to result in a follicular and/or interfollicular pattern of infiltration. These criteria result in a clinically and prognostically relevant separation of patients. They result in an expansion of the LR subgroup with a favourable survival to the detriment of MC. With this, MC shows a worse survival than NS and LR. In MC, patients are older, Bsymptoms, advanced stage disease and eventual death are more frequent. The data support a concept of progression from LR to MC. 


\section{LS 86}

\section{A NOVEL DENDRITIC CELL TYPE FROM HUMAN LYMPHOID TISSUE EXPRESSES AIRE (AUTOIMMUNE REGULATOR)}

P.L. Poliani ${ }^{1}$, V. Marrella ${ }^{2}$, M. Ravanini ${ }^{1}$, L.D. Notarangelo ${ }^{3}$, A. Villa ${ }^{2}$, P. Peterson $\left.{ }^{4},{ }^{*}\right)$ F. Facchetti ${ }^{1}$

${ }^{1}$ Department of Pathology, University of Brescia, Brescia, Italy; ${ }^{2}$ CNR-ITB IRCSS Humanitas, Rozzano, Italy; ${ }^{3}$ Division of Immunology, Children's Hospital, Harvard Medical School, Boston, USA; ${ }^{4}$ Molecular PathologyGMP, University of Tartu, Tartu, Estonia

Self-reactive T-cells from the developing T-cell repertoire are eliminated in the thymus by central tolerance (CT) mechanisms, a process predominantly mediated by medullary epithelial cells expressing AIRE. Although remarkably efficient, few potentially autoreactive T-cells escape CT selection and reach the periphery, where peripheral tolerance (PT) induction is then required to prevent autoimmunity. Recent data (Lee et al., Nat Immunol 2007; 8(2):181-90) have shown that "stromal" cells in murine lymph nodes (LN) express AIRE and could play a role in the induction of PT. Using immunohistochemistry, we investigated AIRE expression in a large number of different human lymphoid and non-lymphoid tissues and characterized the phenotype of AIRE+ cells.

AIRE+ cells were exclusively identified in LN (with predilection of abdominal LN), tonsils and mucosal-associated lymphoid tissues. AIRE + cells were rare and localized in the paracortical area, close to high endothelial venules. Double immunohistochemistry showed that AIRE+ cells consistently co-expressed membranous HLADR and fascin (that highlight their dendritic morphology), and about $50 \%$ of them were positive for S100 and DC-LAMP/CD208, while only a few AIRE + cells also expressed CD11c. Antigens of the lymphoid/ myeloid lineages (including CD45RB, CD45RA, CD45RO, CD34, CD163, CD68, CD123, CXXL13/BCA1), stromal and endothelial cells (smooth muscle actin, desmin, vimentin, CD34, Factor-VIIIRA), and cytokeratins 8/18/19 (CAM5.2) were negative. Cells with a similar phenotype were found in the thymic medulla. AIRE staining on cytospins obtained from magnetic beads cell sorting from mesenteric LNs confirmed the presence of a population of CD45-/ HLA-DR+/AIRE+ cells. On FACS analysis the CD45- sorted cells comprise about $5 \%$ of the total cell population and HLA-DR ${ }^{\text {hi+ }}$ cells represent about $2.3 \%$ of this CD45- fraction. Taken together, these data show that human lymphoid tissues contain a novel rare subset of dendritic cells that express AIRE, partially show an activated phenotype (DC-LAMP+, HLA-DR+) and are possibly involved in PT.

\section{LS 87}

\section{FOXP3-POSITIVE REGULATORY T-CELLS IN CUTANEOUS T-CELL LYMPHOMAS}

*Lise Mette Gjerdrum ${ }^{1}$, Anders Woetmann ${ }^{2}$, Niels Odum ${ }^{2}$, Elisabeth Ralfkiaer ${ }^{1}$

${ }^{1}$ Depatment of Pathology, Rigshospitalet, Copenhagen University Hospital, ${ }^{2}$ Institute for Medical Microbiology and Immunology and Institute of Molecular Microbiology, University of Copenhagen, Copenhagen, Denmark.
Background: FOXP3 is regarded as a unique marker for CD4+ CD25+ regulatory T-cells (Tregs). In solid tumours, high numbers of Tregs are associated with a poor prognosis. Knowledge about the implications of Tregs for the behaviour of haematological malignancies is more limited. We studied 86 skin biopsies from patients with Mycosis Fungoides (MF) and cutaneous T-cell lymphoma (CTCL) unspecified for the expression of FOXP3 on tumour cells and tumour-infiltrating Tregs. In addition, 27 cases with CD30-positive lymphoproliferations (14 cases of lymphomatoid papulosis (LyP), 13 primary cutaneous anaplastic large cell lymphoma (cALCL) were stained with FOXP3.

Results: Labelling of more than $10 \%$ of the neoplastic cells was seen in two cases classified as aggressive epidermotropic CD8+ cytotoxic CTCL and CALCL. Another 7 cases contained only very occasional weakly positive tumour cells. In the remaining cases, the neoplastic infiltrate was FOXP3-negative. By contrast, all biopsies showed varying numbers of strongly FOXP3-positive tumour-infiltrating Tregs. MF with early or infiltrated plaques had significantly higher numbers of FOXP3-positive Tregs than CTCL unspecified or advanced MF with tumours or transformation to large cell lymphoma. Survival analysis demonstrated that increasing numbers of FOXP3-positive Tregs were associated with improved survival in both MF and CTCL unspecified. Similar results were obtained in the 27 cases of primary cutaneous CD30-positive lymphoproliferations. In these conditions, the proportion of Tregs was significantly higher in LyP than in cALCL.

Conclusion: In conclusion, our data indicate that the presence of FOXP3-positive Tregs in CTCL is associated with disease stage and patient survival.

\section{LS 88}

\section{A CASE OF NODULAR LYMPHOCYTE PREDOMINANT HODGKIN LYMPHOMA FOLLOWED WITHIN A MONTH IN THE SAME SITE BY T-CELL AND HISTIOCYTE RICH DIFFUSE LARGE B- CELL LYMPHOMA}

\author{
*S Nikulshin ${ }^{1}, \mathrm{U}$ Mickys $^{2}$, L Griskevicius ${ }^{2}$ \\ ${ }^{1}$ University Children's Hospital, Riga, Latvia \\ ${ }^{2}$ Vilnius University Hospital, Lithuania
}

A 15 y.o. boy presented with enlargement of right axillary lymph nodes for about 3 weeks without signs of infection or B-symptoms; a week before biopsy the nodes had begun to grow faster. USG showed a chain of several deep and more superficial axillary lymph nodes $2 \times$ $3 \mathrm{~cm}$ in diameter. No other sites were found. One of the nodes was excised; histological diagnosis was nodular lymphocyte predominant Hodgkin lymphoma (NLPHL).

The patient was hospitalised for staging and treatment one month after the biopsy. At admittance USG showed polycyclic hyperdense conglomerate $8 \times 9 \mathrm{~cm}$ in the same right axillar region that included deep, superficial, intrapectoral and paramamillar lymph nodes. Still no other sites were visible. No B-symptoms. A large $6 \mathrm{~cm}$ superficial node was excised. Histologically the second biopsy was morphologically and phenotypically different from the first one; histological diagnosis was T-cell and histiocyte rich diffuse large B-cell lymphoma (TCRBCL, CD30 positive. Bone marrow biopsy showed minimal specific involvement. Retrospectively, similar fields could be found in diffuse areas of the first biopsy, too. Clonality studies are in progress. 
The patient was treated according to NHL-BFM-95 (B-cell branch) and is currently in complete remission for 1 year, PET-negative.

Discussion. Occasional cases of nodular lymphocyte predominant Hodgkin lymphoma progressing to TCRBCL after treatment have been described, but simultaneous occurrence seems to be extremely rare. The case could support the hypothesis of a possible biological relationship between these two entities.

\section{LS 89}

\section{PAX5: A VALUABLE IMMUNOHISTOCHEMICAL MARKER IN THE DIFFERENTIAL DIAGNOSIS OF LYMPHOID NEOPLASMS}

Mohamed M Desouki ${ }^{(1)}$, Ginell R Post ${ }^{(1)}$, Daniel Cherry ${ }^{(2)}$, John Lazarchick ${ }^{(1)}$ ${ }^{(1)}$ Department of Pathology \& Laboratory Medicine, Medical University
of South Carolina, Charleston, South Carolina, ${ }^{(2)}$ Department of Pathol-
ogy, Trident Medical Center, Charleston, South Carolina, USA

Background: The grey zone lymphomas constitute a diagnostic challenge due to overlap of morphologic features and antigen expression. PAX5 is a transcription factor which plays an important role in B cell differentiation and proliferation. PAX5 is normally expressed throughout $\mathrm{B}$ cell maturation and is detectable in poorly differentiated B cell neoplasms that fail to express CD45 or pan B-cell markers (CD20 and CD79a). Our results highlight the beneficial role of including PAX5 in an immunohistochemical panel to differentiate lymphoid neoplasms of B cell origin, especially Hodgkin lymphoma (HL) and anaplastic non-Hodgkin lymphoma.

Purpose of study: To assess PAX5 expression in previously diagnosed B- and T- lymphoid neoplasms and undifferentiated tumors. Lymph node, bone marrow and soft tissue tumor sections from 107 archived paraffin blocks were immunohistochemically stained using a monoclonal antibody to PAX5. The corresponding $\mathrm{H}$ and $\mathrm{E}$ stained sections were reevaluated for the presence of malignancy and PAX5 immunoreactivity in neoplastic cells scored.

Results: PAX5 staining was positive in the following cases: $85 \%$ (34/ 40) HL, $100 \%$ (15/15) DLBCL, 100\% (2/2) small B cell lymphomas and $100 \%(5 / 5)$ B-ALL and biphenotypic leukemias with B cell lineage. PAX5 was not detected in anaplastic large cell lymphoma (ALCL; $\mathrm{n}=14$ ), T-LBL and biphenotypic leukemias with $\mathrm{T}$ cell lineage $(n=5)$, AML $(n=3)$, carcinoid tumor $(n=5)$, melanoma $(n=3)$, and undifferentiated/metastatic tumors $(n=8)$. Non-neoplastic bone marrow sections $(n=7)$ showed scattered positive nuclear staining in small B lymphocytes. The detection of PAX5 immunoreactivity resulted in the reclassification of two cases of ALCL to HL.

Conclusion: The inclusion of PAX5 in a panel of immunohistochemical stains is valuable in differentiating CD30 positive $\mathrm{T}$ cell lymphomas such as ALCL, from B cell neoplasms (e.g. HL and DLBCL). Furthermore, absence of PAX5 expression is helpful to rule out non lymphoid tumors with anaplastic cell morphology (undifferentiated and metastatic tumors).

\section{LS 90}

\section{INTERPHASE FISH ANALYSIS ON "STARRY-SKY" APPEARANCE IN PRIMARY CNS LYMPHOMA}

Saitama Medical University, Saitama, Japan

*Hidekazu Kayano, Eiichi Asami, Toshinori Nagai, Keisuke Ishizawa, Shio Shimada, Eiichi Arai, Takanori Hirose

Backgrounds: According to the WHO classification, the vast majority of primary central nervous system lymphoma (PCNSL) is categorized as diffuse large B-cell lymphoma (DLBCL), whereas Burkitt lymphoma (BL) rarely presents as a PCNSL. In PCNSL, perivascular infiltration of lymphoma cells is commonly observed, but diffuse growth area containing scattered tingible-body macrophages, resembling "starry-sky" appearance of $\mathrm{BL}$, is also often observed. Although some extracranial DLBCLs with or without BL-like morphology show MYC abnormalities as seen in $\mathrm{BL}$, little information is available on cytogenetic changes in PCNSL.

Purpose of the study: In order to get more insights into the histological resemblance of PCNSL to BL, we conducted morphological and interphase FISH analysis on paraffin-embedded sections from eleven patients with PCNSL.

Results: All cases were adult (age, 55 to 75 years) and immunocompetent. Histological diagnosis in each case was DLBCL. In addition to perivascular infiltration, "starry-sky" appearance mimicking BL was commonly observed in all but one case presenting with a spinal cord tumor. Immunophenotyping analysis was performed and confirmed an activated B-cell profile of PCNSL (CD20+, CD79a+, CD10-, BCL2+, BCL6+/-, MUM1+). MIB1 (Ki67) staining ranged from 50 to $100 \%$. Using paraffin sections, interface FISH analysis with break-apart rearrangement probes for BCL6 and MYC genes was performed and revealed BCL6 breakage in 2 cases. MYC abnormality was not detected in any of the cases analysed.

Conclusion: In spite of histological resemblance to BL, PCNSLs in this study showed different immunophenotypic and molecular features from those seen in BL. "Starry-sky" appearance in PCNSL may be surrogate for molecular mechanisms involving genes other than $M Y C$.

\section{LS 91}

\section{THE SYNCYTIAL VARIANT OF NODULAR LYMPHOCYTE PREDOMINANCE HODGKIN LYMPHOMA REPORT OF A CASE AND REVIEW OF THE LITERATURE}

Drakos Elias*, L. Jeffrey Medeiros, Vassiliki Leventaki, Claudiu V. Cotta, Fransisco Vega, and George Z. Rassidakis

Department of Hematopathology, The University of Texas M.D. Anderson Cancer Center, Houston, Texas, USA

Background: Nodular lymphocyte predominance Hodgkin lymphoma (NLPHL) shows immunohistologic and clinical features distinct from classical Hodgkin lymphoma (cHL). However, in addition to typical histology, variant histologic patterns of NLPHL have been recently described, which may have clinical significance.

Purpose: We present a case of NLPHL in a 41-year-old man with long clinical follow-up. We discuss an uncommon histologic variant of 
NLPHL mimicking syncytial variant of nodular sclerosis cHL and we review the literature.

Results: The patient presented with an inguinal mass, and stage IIA disease. Histology revealed a high-grade lymphoma with a distinctly nodular pattern, and clustering of a variable number ( 6 to more than 100) of L\&H-like cells within the nodules associated with heavy neutrophil infiltrates, areas of internodular fibrosis and, focally, lacunar morphology of the neoplastic cells. Immunophenotypic analysis demonstrated that the neoplastic cells were CD30-, CD15-, CD45+, CD20+, PAX5+ EMA+, BCL6+, BCL2-, PU.1-, and LMP1-. The neoplastic cells also were found to be cJUN-, JUNB-, and Id2+. CD21+ follicular dendritic meshwork defined all nodules. Treatment of this patient with 4 cycles of ABVD and involved field irradiation (30 Gy) resulted in complete response. The patient remains without evidence of disease until today, 14 years after initial diagnosis. In the literature, cases with nodular and clustered variant pattern of NLPHL, but without fibrosis and neutophil infiltrates, are extremely rare, and show excellent survival.

Conclusions: Recognition of uncommon histologic variants of NLPHL would facilitate accurate differential diagnosis and enable future clinical studies to address the optimal treatment of these patients.

\section{LS 92}

\section{INCREASING INCIDENCE OF OPHTHALMIC LYMPHOMA IN DENMARK FROM 1980 TO 2005}

*Sjö LD ${ }^{1}$, Ralfkiaer $\mathrm{E}^{2}$, Prause $\mathrm{JU}^{1}$, Petersen $\mathrm{JH}^{3}$, Madsen $\mathrm{J}^{4}$, Pedersen $\mathrm{NT}^{5}$, Heegaard $\mathrm{S}^{1}$

1: Eye Pathology Institute,Dept. of Neuroscience and Pharmacology, University of Copenhagen, Copenhagen, Denmark

2: Department of Pathology, Rigshospitalet, Copenhagen, Denmark

3: Department of Biostatistics, University of Copenhagen, Copenhagen, Denmark

4: Department of Hematology, Aalborg Hospital, University of Aarhus, Aarhus, Denmark

5: Department of Pathology, Odense University Hospital, Odense, Denmark

Background: Ophthalmic lymphoma (lymphoma localized in the ocular region, i.e. orbit, lacrimal gland, lacrimal sac, eyeball, conjunctiva or eyelid) constitutes approximately $10 \%$ of all extranodal NHL, and lymphoma is the most common orbital malignancy. Most lymphomas arising in the ocular region are low-grade B-cell lymphomas, with a high frequency of extranodal marginal zone Bcell lymphoma (MALT lymphoma)

Knowledge of both the geographic distribution and time trends of ophthalmic lymphoma may be helpful in formulating new aetiologic hypotheses. However, the co-existence of multiple NHL classification systems (e.g., Kiel, Lukes-Collins, Rappaport, Working Formulation) has complicated epidemiologic research of NHL, and incidence data on ophthalmic lymphomas are sparse. Two reports based on register data indicate that the incidence of ophthalmic lymphoma in the United States of America is increasing at an even higher rate than NHL in general. The implementation of the REAL classification and the WHO-classification system provides new possibilities to perform reliable epidemiological studies on ophthalmic lymphoma.

Purpose: To evaluate patient characteristics and incidence of ophthalmic lymphoma in Denmark during the period 1980 to 2005.
Results: A total of 228 patients with a histologically verified diagnosis of ophthalmic lymphoma were included. There was an equal distribution of men and women. The most frequent lymphoma subtype was extranodal marginal zone B-cell lymphoma (MALT lymphoma, 55.5\%) and most cases were located in the orbit $(56.8 \%)$. High-grade lymphoma subtypes were found more frequently in men than in women. Incidence rates were highly dependent of patient age. For all ages a statistically significant annual average increase of $3.4 \%$ during the 26-year-period was found. This was primarily due to a rise in incidence of MALT lymphoma.

Conclusions: In the Danish population ophthalmic lymphoma consists primarily of orbital MALT lymphoma. Although a rare disease of elderly patients, the incidence of ophthalmic lymphoma is increasing rapidly.

\section{LS 93}

\section{miRNA PROFILING AND MIRNA TARGET GENES IN HODGKIN LYMPHOMA}

Anke van den Berg, Lu Ping Tan, Johan Gibcus, Geert Harms, Rikst Nynke Schakel, Tjasso Blokzijl, Rogier Reijmers, Steven pals, Ralf Küppers, Peter Möller, Sibrand Poppema, Bart-Jan Kroesen

Department of Pathology, University Medical Center Groningen and University of Groningen, The Netherlands. Department of pathology, Academic Medical Center, University of Amsterdam, The Netherlands, Department of Cell Biology, University of Duisberg-Essen, Germany, Institute of Pathology, University of Ulm, Germany

Background: Based on several recent publications it has become evident that miRNAs play an important role in the pathogenesis of B cell lymphoma. MiRNAs inhibit protein translation of multiple targets based on binding to the 3'-UTR.

Purpose: Elucidate the role of miRNAs in Hodgkin lymphoma (HL). Methods: Microarray analyses were performed to generate miRNA profiles of HL. Validation of the most abundant and differentially expressed miRNAs was performed by miRNA-ISH and qRT-PCR. Identification of miRNA target genes by immunoprecipitation (IP) of the AGO2-RISC complex followed by microarray analysis to identify the target genes enriched in the RISC-IP fraction. Target gene validation was performed in various HL cell lines using a luciferase reported system.

Results: miRNA profiling indicated that the overall expression levels were markedly increased in HL. Specifically, most members of the oncogenic C13ORF25 cluster, miR-21 and miR-155 were highly expressed. For a selection of the miRNAs detected in the profiling, expression in HRS cells could be confirmed by RNA-ISH. 7/13 miRNAs, differentially expressed between $\mathrm{cHL}$ and PMBL \& $\mathrm{cHL}$ and EBV transformed B cells were confirmed as differentially expressed miRNAs in a panel of 33 cell lines. RISC-IP in two HL derived cell lines followed by gene expression profiling to identify the miRNA target genes enriched in the IP fraction resulted in experimental identification of approximately 100 consistent target genes. Experimental validation in a luciferase reporter assay resulted in reduced luciferase activity for five miR-155 target genes (ZIC3, ZNF537, AGTR1, IKBкE and KGF) and two miR-17 target genes (GPR137B and RBJ).

Conclusions: HL displays a unique miRNA expression signature, with high expression of miR-155, several members of the C13ORF25 
cluster, and miR-21 and low expression of miR-150. Five genes were identified as putative miR-155 targets and two as putative targets for miR-17 in HL.

\section{LS 94}

\section{ROSETTING T CELLS IN HL, CHARACTERIZATION AND CROSS-TALK WITH HRS CELLS}

Anke van den Berg, Yue Ma, Tjasso Blokzijl, Geert Harms, Çiğdem Atayar, Sibrand Poppema, Lydia Visser

Department of Pathology \& Laboratory Medicine, University Medical Center Groningen, University of Groningen, Groningen, The Netherlands

Background: Hodgkin and Reed-Sternberg (HRS) cells in Hodgkin lymphoma (HL) secrete factors that interact with inflammatory background cells and may serve as biomarkers for disease activity. The majority of the infiltrating cells are T-cells and the cross-talk of these cells with the HRS cells is important for the survival of the HRS cells. The rosetting CD4+CD26- T-cells are characteristic for classical HL.

Purpose: To characterize the rosetting CD4+CD26- T-cells from cHL and to detect potentially new players involved in the cross-talk by characterizing the secretome of HRS cells.

Methods: Quantitative RT-PCR for 46 genes on stimulated and resting CD4+CD26- and CD4+CD26+ $\mathrm{T}$ cell subsets from $\mathrm{cHL}$ and tonsil. Identify proteins in cell culture supernatant of four HL cell lines using 1DGE followed by in-gel trypsin digestion and protein identification using LC-MS/MS.

Results: CD4+CD26- T-cells in HL showed higher mRNA levels of CD25, CTLA4, OX40 and CCR4 compared to LN, supporting a Treg cell type. Based on high IL17 levels Th17 cells may also exist in HL. Upon stimulation in vitro, mRNA levels of most cytokines remained low indicating an anergic character for the CD4+CD26- T-cell subset. In total, 368 secreted proteins were identified in the HL cell culture supernatant, including 37 proteins involved in immune response, including TARC known to attract CCR4 positive Treg and Th2 cells; IL25 inhibiting Th1 and Th17 cells, but stimulating Th2 cells; MIF, blocking cytotoxic T cells. Most proteins were validated in HL cell lines and patient tissue. Seven proteins revealed significantly elevated levels in patient plasma compared with healthy controls.

Conclusions: Cytokine profiling of $\mathrm{CD} 4+\mathrm{CD} 26-\mathrm{T}-$ cells in $\mathrm{cHL}$ indicates that they are anergic Treg cells, explaining the lack of an effective anti-tumor response in HL. Absence of Th1 and cytotoxic T cell responses might be caused by IL25 and MIF produced by HRS cells.

\section{LS 95}

\section{TONSILLAR EBV POSITIVE DIFFUSE LARGE B CELL} LYMPHOMA WITH ANAPLASTIC PHENOTYPE

*Metka Volavšek, M.D., Ph.D.

Institute of Pathology, Faculty of Medicine, University of Ljubljana, Ljubljana, SLOVENIA

Background: Etiology of primary tonsillar lymphoma which is usually of the diffuse large B cell (DLBL) type is mostly unknown. In minority of patients an underlying immune deficiency can be proven and lymphomas are then mostly associated with Epstein-Barr virus (EBV).

Purpose of the study: A case of bilateral asymetrical tonsillar enlargement in a promiscuous patient with anamnesis of hepatitis B is reported.

Results: In a patient with cough and pharyngeal irritation of a year duration asymetrical tonsillar enlargement was discovered, with more painful somewhat larger left tonsil and one painful enlarged left neck lymph node. Bilateral tonsillectomy was performed, the neck lymph node was not excised.

Histology of both tonsils was similar. In addition to reactive follicular hyperplasia there were areas of surface ulceration with underlying mostly interfollicular proliferation of medium sized and large anaplastic cells which were focally invading the follicles. Immunophenotype of anaplastic cells was: CD20+, CD30+, CD3-, CD4-, CD8-, CD5-/+, CD56-, perforin-, CD23-, cyclin D1-; in situ hybridization for kappa and lamba light chains-, EBER+, Ki-67 cca $70 \%$. Molecular-genetic analyses were not performed. A diagnosis of DLBL with anaplastic phenotype, EBV positive, probably originating from lymphomatoid granulomatosis grade III was rendered. Additional serology suggested by pathologist proved patient's HIV positivitiy and the staging procedures determined clinical stage IIA. After 4 cycles of chemotherapy (CHOP and Neupogen) severe neutropenia $\left(0,2 \times 10^{6} \mathrm{Ne} / \mathrm{g}\right)$ developed and 14 days ago the chemotherapy was temporarily disconnected.

Conclusion: Differential diagnosis (DD) of tonsillar neoplasms is wide and in addition to malignant lymphomas includes epithelial neoplasms, especially lymphoepithelial carcinoma (EBV positive). EBV positivity (EBER) narrows the DD of lymphoid neoplasms to various types of malignant lymphoma occuring in immunocompromised patients, but in noncompromised individuals Burkitt lymphoma, lymphomatoid granulomatosis, DLBL and T cell lymphoma-nasal type are the prime candidates. To reach a definite diagnosis testing for EBV infection and anamnestic data of immune deficiency/HIV infection are of outmost importance.

\section{$\underline{\text { LS } 96}$}

\section{JUNB / CD30 SIGNALING CONTRIBUTES TO CELL CYCLE PROGRESSION THROUGH REGULATION OF p14 AND p21 IN ANAPLASTIC LARGE CELL LYMPHOMA}

V. Atsaves ${ }^{1,2}$, L. Lekakis ${ }^{1}$, M. Feretzaki ${ }^{1}$, V. Leventaki ${ }^{1}$, Ch. Liakou ${ }^{1}$, E. Drakos ${ }^{1}$, F.X. Claret ${ }^{1}$, D. Jones ${ }^{1}$, L.J. Medeiros ${ }^{1}$, E. Patsouris ${ }^{2}$, G.Z. Rassidakis ${ }^{1,2}$

${ }^{1}$ The University of Texas M.D. Anderson Cancer Center, Houston, TX

${ }^{2}$ National and Kapodistrian University of Athens, Greece

Background: JunB is a member of the jun family of AP-1 transcription factors involved in cell proliferation and apoptosis. JunB positively regulates $\mathrm{CD} 30$ gene expression at the transcriptional level in Hodgkin lymphoma and anaplastic large cell lymphoma (ALCL) cells. Purpose: We hypothesized that JunB may contribute to uncontrolled cell cycle progression through CD30 signalling in ALCL.

Results: Two ALK+ ALCL cell lines, Karpas299 and SUDHL-1, as well as a control cell line (Jurkat) were used. Silencing of JunB using siRNA in ALCL cells resulted in decreased AP-1 binding activity assessed by EMSA and a significant decrease in S-phase assessed by 
BrdU incorporation studies. These changes were associated with increased levels of the CDK inhibitors p21 and p14 with simultaneous decrease of the unphosphorylated fraction of $\mathrm{Rb}$ and expression levels of cyclins A, D2 and D3. Silencing of JunB in ALCL cells also resulted in decreased CD30 expression and CD30 promoter activity using luciferase assay. In addition, silencing of CD30 using siRNA in ALCL cells led to decreased AP-1 binding activity, which was associated with changes in cell cycle and levels of p 21 and p14 similar to those seen after JunB silencing. Similar findings were observed after treatment of ALCL cells with an anti-CD30, antibody, SGN30, which is currently tested in clinical trials.

Conclusions: These findings suggest that JunB/CD30 signalling controls cell cycle progression through deregulation of cell cycle inhibitors representing a novel therapeutic target in ALCL.

\section{LS 97}

\section{THE DIAGNOSTIC DILEMMA OF SPLENIC T CELL / HISTIOCYTE RICH LARGE B CELL LYMPHOMAS}

Oner Dogan ${ }^{1}$, Mehmet Agan ${ }^{1}$, Gulsah Kaygusuz ${ }^{2}$, Aptullah Haholu ${ }^{3}$, Aysegul Uner ${ }^{4}, *$ Isinsu $\mathrm{Kuzu}^{2}$

${ }^{1}$ Istanbul University School of Medicine Dept of Pathology Istanbul, ${ }^{2}$ Ankara University School of Medicine Dept of Pathology Ankara, ${ }^{3}$ Gulhane Medical Academy Haydarpasa Teaching Hospital Istanbul, ${ }^{4}$ Hacettepe University School of Medicine Dept of Pathology Ankara.

Background: Micronodular $\mathrm{T}$ cell/ histiyocyte rich large $\mathrm{B}$ cell lymphoma of the spleen (M- TCRBCL-S) is a unique lymphoma which poses diagnostic difficulties. The clinical presentation and the symptoms that are similar to some infectious or autoimmune hematological diseases may cause the delay in diagnosis.

Aim: The aim of this study is to examine the clinical and histopathological characteristics of the lymphomas diagnosed as $\mathrm{M}$ TCRBCL-S in 12 of our cases and discuss the difficulties in diagnosis.

Results: The median age of the patients were 37,5 (ranging between 19-63) with female dominance (8 Female / 4 Male). Splenomegaly, pancytopenia, weight loss and fever were the most frequent symptoms or findings. One patient had developed the disease, under follow up for common variable immune deficiency syndrome (CVID). Except for one case all the patients had bone marrow infiltration with increased $\mathrm{T}$ lymphocytes, histiocytes with or without few neoplastic B cells. All patients had lymph node involvement in splenic hilus. Liver involvement was present in 6 of 7 cases in whom liver biopsies were available. On gross examination of the splenectomy specimens, the cut surfaces were congested with millimetric nodules. The neoplastic large cells were all CD20 positive and half of the cases showed faint CD30 positivity whereas all were CD15 negative. As a first sample examined, the bone marrow biopsy findings for all cases were not found sufficient to render the diagnosis of B cell lymphoma. Half of the cases were treated with steroids before splenectomy.
Conclusion: Bone marrow and/or liver biopsies were the most common initial specimens examined in our series of M- TCRBCL$\mathrm{S}$. These biopsies were suspicious but not diagnostic because of sparce neoplastic B lymphocytes present in a reactive background. Steroid treatment could have also contributed to the interpretation difficulties in some cases.

\section{LS 98}

\section{SPECIFIC EXPRESSION OF MIR-17-5P AND MIR-127 IN TESTICULAR AND CENTRAL NERVOUS SYSTEM DIFFUSE LARGE B-CELL LYMPHOMA}

\author{
${ }^{* 1}$ Jan-Lukas Robertus, ${ }^{1}$ Geert Harms, ${ }^{1}$ Tjasso Blokzijl, ${ }^{1}$ Marije \\ Booman, ${ }^{3}$ Daphne de Jong, ${ }^{2}$ Gustaaf van Imhoff, ${ }^{1}$ Stefano Rosati, \\ ${ }^{1}$ Ed Schuuring, ${ }^{1}$ Philip Kluin, ${ }^{1}$ Anke van den Berg \\ ${ }^{1}$ Department of Pathology \& Molecular Biology, ${ }^{2}$ Department of \\ Hematology, University Medical Center Groningen, University of \\ Groningen, Groningen, The Netherlands \\ ${ }^{3}$ Department of Pathology, Netherlands Cancer Institute, Amsterdam, \\ The Netherlands
}

Background: Recent studies have shown that certain non-coding short RNAs (22nt) called miRNAs play an important role in diffuse large B-cell lymphomas (DLBCL). Patients with DLBCL have a great diversity in both clinical characteristics, site of presentation and outcome. Purpose of the study: To validate the differential expression in GCB and non-GCB DLBCL and to determine to what extent the primary site of presentation is associated with the miRNA expression profile. Materials and Methods: We studied 50 cases of de novo DLBCL for the expression of 15 miRNAs (miR-15a, miR-15b, miR-16, miR-173p, miR-17-5p, miR-18a, miR-19a, miR-19b, miR-20a, miR -21, miR-92, miR-127, miR-155, miR-181a and miR-221). Apart from 19 nodal cases without extranodal dissemination (stage I and II) we selected two groups with unambiguous stage I and II extranodal presentation, nine cases of primary central nervous system (CNS) DLBCL and 11 cases of primary testicular DLBCL as well as 11 other primary extranodal DLBCL. All cases were analyzed with qRT-PCR. In situ hybridization (ISH) performed for the most differentially expressed miRNAs demonstrated that they were expressed in tumor cells and not in background cells.

Results: MiR-21 and miR-19b showed the highest expression levels. No significant differences were seen between GCB and non-GCB DLBCL in the total or nodal DLBCL group for any of the 15 miRNAs. Two miRNAs showed significant differences in expression levels for DLBCL subgroups according to site of presentation. MiR$17-5 p$ showed a significant higher expression level in CNS DLBCL compared to testicular and nodal DLBCL $(p<0.05)$. MiR-127 levels were significantly higher in testicular DLBCL when compared to CNS and nodal DLBCL $(p<0.05)$. ISH for miR-127 and miR-17-5p showed high expression in tumor cells and not in reactive or normal cells.

Conclusion: We conclude that location of DLBCL is an important factor in determining differential expression of miRNAs. 


\section{LS 99}

\section{THE ONCOGENIC MIR-17-92 SHOWS B-CELL MALIGNANCY SPECIFIC EXPRESSION PATTERNS}

${ }^{* 1}$ Jan-Lukas Robertus, ${ }^{1}$ Miao Wang, ${ }^{1}$ Lu Ping Tan, ${ }^{1}$ Veera Nadarjan, ${ }^{1}$ Geert Harms, ${ }^{1}$ Evert-Jan Boerma, ${ }^{3}$ Rogier Reijmers, ${ }^{3}$ Steven Pals, ${ }^{1}$ Stefano Rosati, ${ }^{1}$ Ed Schuuring, ${ }^{2}$ Gustaaf van Imhoff, ${ }^{1}$ Philip Kluin, ${ }^{1}$ Anke van den Berg

${ }^{1}$ Department of Pathology and Molecular Biology and ${ }^{2}$ Department of Hematology, University Medical Center Groningen, University of Groningen, The Netherlands.

${ }^{3}$ Department of Pathology, Academic Medical Center, University of Amsterdam, The Netherlands

Introduction: The primary miR-17-92 (pri-miR-17-92) microRNA (miRNA) polycistron or C13ORF25 has recently immerged as an important oncogene in various cancers. C13ORF25 is located at 13q31-32 and has been shown to be amplified and over expressed in various non-Hodgkin lymphoma (NHL) subtypes.

Purpose of the study: To determine differences in processing and or stability between separate NHL of the seven individual miRNAs present in C13ORF25 and to compare if the absolute abundance of each miRNA is specific for different NHL.

Materials and Methods: C13ORF25 and miR-17-92 expression levels were determined using qRT-PCR in 121 cases of NHL which included diffuse large B-cell lymphoma (DLBCL), chronic lymphatic leukemia (CLL), pediatric Burkitt lymphoma (BL) and mantle cell lymphoma (MCL). In addition we also analyzed NHL cell lines and three B-cell subsets.

Results: Analysis of C13ORF25 showed significantly higher expression levels $(\mathrm{p}<0.05)$ in BL and DLBCL. The expression pattern of miR-17-92 showed that miR-92 was the most highly expressed miRNA in MCL, CLL and BL cases but also in the cell lines and normal B-cell subsets. Interestingly, in the DLBCL cases miR-19b expression levels were the highest. Unsupervised hierarchical clustering of the absolute abundance of each miRNA in the 121 NHL cases revealed three main clusters. The first and second cluster contained respectively all MCL cases and all CLL cases. The third cluster contained three subgroups. The first subgroup contained most of the DLBCL cases. The second and third subgroup contained BL cases characterized by respectively low expression of miR-17-5p and high expression of miR-92.

Conclusion: The four NHL each showed a different expression pattern for the seven miRNAs and C13ORF25 which may be caused by differences in processing and or stability. The characteristic clustering pattern of the four malignancies showed that the abundance for each separate miRNA is specific for the four NHL.
LS 100

BENDAMUSTINE (TREANDA) CYTOTOXICITY IN B-CELL NEOPLASMS REQUIRES PRODUCTION OF REACTIVE OXYGEN SPECIES AND CASPASE-UNRELATED PROCESS IRRESPECTIVE OF P53 STATUS

* Gaël Roué, ${ }^{1}$ Mónica López-Guerra, ${ }^{1}$ Pierre Milpied, ${ }^{1}$ Patricia PérezGalán, ${ }^{1}$ Neus Villamor, ${ }^{1}$ Emili Montserrat, ${ }^{2}$ Elias Campo, ${ }^{1}$ and Dolors Colomer $^{1}$

${ }^{1}$ Hematopathology Unit, Department of Pathology and ${ }^{2}$ Department of Hematology, Hospital Clínic, Institut d'Investigacions Biomèdiques August Pi i Sunyer (IDIBAPS), University of Barcelona, Barcelona, Spain.

Background: chronic lymphocytic leukaemia (CLL) and mantle cell lymphoma (MCL) are two types of B-cell lymphoid neoplasms characterized respectively by a relatively indolent natural history and an aggressive course. These two diseases remain incurable. Bendamustine hydrochloride (TREANDA) is a multifunctional, alkylating agent with a purine-like ring system that exhibits activity in multiple cancer models, but whose mechanism of action is partially unknown. Purpose of the study: to analyse the apoptotic pathways activated by bendamustine in CLL and MCL, together with the relevance of p53 mutation in determining the response of malignant $\mathrm{B}$ cells to this drug alone or combined with nucleoside analogues.

Methods: 13 CLL/MCL cell lines and primary tumour cells from $8 \mathrm{MCL}$ and $25 \mathrm{CLL}$ patients were cultured for up to 24 hours with bendamustine (Treanda, provided by Cephalon Inc.). Cytotoxic assays, flow cytometry, immunofluorescence and western blot analysis of DNA-damage response pathway and apoptosis-related factors.

Results: we show that bendamustine exerts a cytotoxic effect on most CLL and MCL primary cells and cell lines, irrespective of ZAP-70 expression and p53 status. Bendamustine cytotoxicity was mediated by the generation of reactive oxygen species, and p53-dependent and p53-independent triggering of the intrinsic apoptotic pathway involving up-regulation of PUMA and NOXA, conformational activation of BAX and BAK, and cytosolic release of caspase-related and caspaseunrelated mitochondrial apoptogenic proteins. More importantly, bendamustine was found to act synergistically with nucleoside analogues, this combination being effective in non-functional p53 CLL and MCL cases resistant to standard chemotherapy.

Conclusions: our findings support the use of bendamustine as a therapeutic agent for CLL and MCL and establish the basis for its combination with conventional genotoxic agents.

\section{$\underline{\text { LS } 101}$}

\section{ANCILLARY TECHNIQUES IN CYTOLOGICAL DIAGNOSIS OF B-CELL LYMPHOMAS}

*V. Kloboves Prevodnik, Lavrenčak J. Koković I, Novaković S.

Institute of Oncology, Ljubljana, Slovenia

Background: The cytological differentiation between B-cell lymphomas and reactive lymphocytic proliferation based only on microscopic examination of cytological slides is difficult and quite often 
inaccurate. Therefore, ancillary techniques, such as multiparametric flow cytometric immunophenotyping (FCI) and determination of gene rearrangements, should be used to establish a more accurate diagnosis. Purpose of the study: To assess which ancillary technique is more helpful in routine cytological diagnostics of B-cell lymphomas we performed both FCI and PCR-based gene rearrangement analysis in 40 cases where B-cell lymphoma was suspected by microscopical examination of cytological slides. The cytological diagnosis was verified by surgical biopsy and histological examination in 11 and by clinical follow-up data in 29 cases.

Results: The final diagnosis was B-cell lymphoma in 11 and reactive lymphocytic proliferation in 29 cases.

In B-cell lymphomas, FCI detected monoclonality in 9 cases. In the remaining two cases, light chains were not detected or stained nonspecifically. PCR-based clonality analysis displayed monoclonality in only 5 B-cell lymphomas. In one case, monoclonality was suspected and 5 were polyclonal.

In reactive lymphocytic proliferations, FCI displayed polyclonality in 25 and monoclonality in one case. Light chains were not detected in 3 cases. In addition, T-lymphocytes with aberrant immunophenotype were found in 3 cases. PCR-based clonality analysis showed polyclonality in 27 reactive lymphocytic proliferations and monoclonality in two. However, in one case, monoclonality was only suspected.

Conclusions: FCI is more sensitive than PCR-based gene rearrangement analysis in detecting monoclonal B-cells and is therefore considered to be more helpful in routine cytological diagnostics of B-cell lymphomas. PCR-based clonality analysis could be beneficial only when light chains are stained non-specifically or are not detected by FCI.

\section{LS 102}

\section{NON HODGKIN'S LYMPHOMAS}

\section{IN CHILDREN AND TEENAGERS}

\section{K. MEKHELEF- ITATAHINE* , M. BOUFAMA*, K. BOUZID - BENDISARI*}

* : Beni-Messous Pathology Institute, Algiers, Algeria

Background: Among children and teenagers cancers examined in Beni-Messous pathology institute Non Hodgkin's lymphomas constitute the most frequent malignancies. They are high grade lymphoma. Extra nodal sites represent approximatively all the cases, particularly abdominal and mediastinal sites.

Purpose of the study: Our study brings the histopathological types according to the 2001 who's classification, the problems of differential diagnosis helped by markers used and prognosis.

Description of results: Burkitt's lymphoma without the Jaw's location knowed in Algeria with its association to EBV predominates in abdominal sites.

High grade B cell lymphomas are particularly mediastinal but lymphoblastic lymphomas are more rare in our institute.

Conclusion: They are aggressive malignancies, which respond to heavy chemotherapy with good prognosis and out come despite pejorative cases due to the toxicity and infections still noted in our country.

\section{LS 103}

\section{CYCLIN D1 EXPRESSION IN NODULAR LYMPHOCYTE-PREDOMINANT HODGKIN'S LYMPHOMA IS RELATED TO TRISOMY OF THE CYCLIN D1 GENE.}

*Brigiet Hoevenaars $\mathrm{MD}^{1}$, Jos Meijer MD, $\mathrm{PhD}^{2}$, Konnie Hebeda $\mathrm{MD}, \mathrm{PhD}^{1}$, Johan van Krieken $\mathrm{MD}, \mathrm{PhD}^{1}$

${ }^{1}$ Dept of pathology Radboud University Nijmegen Medical Centre, The Netherlands.

${ }^{2}$ Dept of pathology Rijnstate Arnhem Medical Centre, The Netherlands.

Background: Cyclin D1 is a promotor of progression of the cell cycle and its overexpression is mostly observed in mantel cell lymphomas $(>95 \%)$ due to a translocation of the BCL1 locus $(\mathrm{t}(11 ; 14))$. Cyclin D1 expression has also been reported in other B-cell lymphoproliferative diseases (ea chronic lymphocytic leukemia, plasmacytomas, hairy cell leukemia). Coincidentally Cyclin D1 expression was detected in a case of nodular lymphocyte-predominant Hodgkin's lymphoma. Therefore a study for Cyclin D1 expression was performed in 13 cases of nodular lymphocyte predominant Hodgkin's lymphoma, 7 cases of lymphocyte-rich classical Hodgkin's lymphoma and 10 cases of nodular sclerosing Hodgkin's lymphoma. In addition, in two Cyclin D1 positive cases fluorescent in situ hybridization (FISH) was performed for the detection of $\mathrm{t}(11 ; 14)$.

Results: Immunohistochemistry of Cyclin D1 showed nuclear positivity of the tumor cells in 8 of 13 cases of the nodular lymfocyte-predominant Hodgkin's lymphoma. In 2 of 8 Cyclin D1 positive cases FISH was performed, which showed in both cases trisomy of the Cyclin D1 locus but not of the centromere of chromosome 11. There was no $\mathrm{t}(11 ; 14)$. In none of 7 classical lymphocyte-rich Hodgkin's lymphomas and in only 1 of 10 nodular sclerosing Hodgkin's lymphomas Cyclin D1 protein expression was found. FISH analysis in these cases is in progress.

In conclusion: A substantial part of nodular lymphocyte-predominant Hodgkin's lymphomas shows overexpression of Cyclin D1, in this study 8 cases of 13 of which at least 2 show a trisomy of the Cyclin D1 gene. This is in contrast with classical lymphocyte-rich Hodgkin's lymphomas and nodular sclerosing Hodgkin's lymphomas.

\section{LS 104}

\section{IMMUNOPHENOTYPICAL FEATURES OF PRIMARY TESTICULAR DIFFUSE LARGE B-CELL LYMPHOMA}

\author{
Ergin $\mathbf{M}^{*}$, Bağır E, Gönlüșen G
}

\section{Cukurova University Medical School Dept of Pathology, Adana/ TURKEY}

Background: Primary non-Hodgkin's lymphoma of testicle is rare. The most common type is diffuse large B-cell type (DLBCL). Recently, DLBCL has been subclassified as germinal center B-celllike and non-germinal center B-cell types. Such distinction is performed possible using the immunohistochemical expression of CD10, Bcl-6 and MUM1. 
Purpose of the study: The aim of this study was to subclassify primary testicular DLBCL according to this sheme. Immunohistochemical sudy using antibodies against CD10, Bcl-6 and MUM1 were carried out.

Results: We investigated 12 patients with primary testicular DLBCL. Immunohistochemistry showed 0/12 for CD10, 4/12 for Bcl-6, 11/12 for MUM1. The expression pattern of CD10 (-) MUM1 (+) in primary testicular DLBCL corresponded to the nongerminal center B-cell group for 11 cases.

Conclusion: The most primary testicular DLBCL (91.6\%) has a nongerminal center B-cell phenotype. These features might therefore be associated with poor prognosis.

\section{LS 105}

\section{PLASMABLASTIC. LYMPHOMA ASSOCIATED WITH COINFECTION OF BOTH EBV AND HHV-8 IN A HIV SERONEGATIVE PATIENT : A CASE .REPORT}

Emine Dündar ${ }^{1}$, ${ }^{*}$ Serap Isiksoy $^{1}$, Zafer Gulbas ${ }^{1}$, Mukerrem Safali ${ }^{2}$, Aysegul Uner ${ }^{3}$, Isinsu Kuzu ${ }^{4}$

${ }^{1}$ Osmangazi Unversity School of Medicine Dept of Pathology Ankara, ${ }^{2}$ GATA Faculty of Medicine Dept of Pathology Ankara, ${ }^{3}$ Hacettepe University School of Medicine Dept of Pathology Ankara, ${ }^{4}$ Ankara University School of Medicine Dept of Pathology Ankara,

Plasmablastic lymphomas has been described as a variant of diffuse large B-cell lymphoma These lymphomas are often seen in the extranodal sites and HIV positive individuals Tumor consists of plasmacytoid transformed cells with a phenotype more like plasma cells than large lymphoid cells. An exclusive predilection to the male gender and frequent association to EBV and immunodeficiency are known, however the disease spectrum has been expanding with a number of single case reports. We present a female young HIV negative patient . Plasmablastic lymphoma diagnosed at supraclavicular lymph node examination. Tumor cells were CD20,CD3, ALK-1, CD30, Bcl-6, Pax-5, kappa, lambda negative, most of the tumor cells were CD38, CD138, HHV-8 and EBV LMP-1 positive. EBV and HHV8 colocalisation was also confirmed with PCR. We encountered only two other cases in the literature to be associated with coinfection of these two lymphotrophic viruses in HIV negative patients. The patient applied with a complaint of abdominal pain.and leg swelling. Ultrasonographic examination revealed intraabdominal fluid accumulation. Cytological examination of the abdominal fluid suggested lymphoma. She had generalised lympadenopathy, hepatomegaly and bilateral renal involvement.

\section{LS 106}

\section{USE OF A CHIP-BASED MICROFLUIDIC APPARATUS FOR THE DETECTION OF B-CELL CLONALITY USING THE MULTIPLEX BIOMED-2 PCRs}

Anna Bertolaso $^{1 *}$, Patricia J.T.A. Groenen ${ }^{2}$, Annemiek W.M. van Raaij $^{2}$, Francesca Mancini ${ }^{1}$, Marina Montresor ${ }^{1}$, Maria Scardoni ${ }^{1}$, Marco Chilosi ${ }^{1}$, Fabio Menestrina ${ }^{1}$, Aldo Scarpa ${ }^{1}$ and Alberto Zamò ${ }^{1}$

${ }^{1}$ Department of Pathology, University of Verona, Verona, Italy

${ }^{2}$ Dept. of Pathology, Radboud University Nijmegen Medical Centre, Nijmegen, The Netherlands.
Background: The multiplex BIOMED-2 PCRs for detection of B-cell clonality recognize almost all immunoglobulin gene rearrangements by using gene-scanning and capillary electrophoresis (CE) or heteroduplexpolyacrylamide gel electrophoresis. Chip-based microfluidic electrophoresis (CME) represents a viable alternative because of its good discriminative power, sensitivity, speed, ease of use and relative low-cost. Purpose of the study: To test the potential of the CME-technology for B-cell clonality detection.

Results: We tested 54 B-NHL and 15 reactive lymph nodes (RLN) for clonality detection using CME technology (Agilent 2100 Bioanalyzer). Clonal rearrangements were detected in $95 \%(22 / 23)$ non-FL B-NHL cases (18 CLL, 3 DLBCL, 1 SMZL, 1 LPL) and in 90\% (28/ 31) FL-cases. No clonal products were detected in the RLN.

We also compared the use of CME to gene-scanning by CE (ABI-3730; Applied Biosystems) using 11 samples, including: 3 clonal samples, 1 RLN and 6 "borderline" cases that gave ambiguous results using CME. Of the three clonal samples, two were confirmed by Genescanning and CE. One sample that showed a weak clonal rearrangement in the IGH-FR2 PCR by CME was interpreted as polyclonal by CE.

Clonal rearrangements in multiple targets were detected by $\mathrm{CE}$ in $6 / 6$ borderline cases, which was in concordance with the CME method. An isolated IGK-DE rearrangement was detected in the RLN by CE, however was not considered clonal by CME. We propose a rule for interpreting CMEpeak patterns. In addition, a time and cost analysis has been performed.

Conclusion: CME may be a viable alternative analysis method for assessment of the BIOMED-2 PCRs. It shows a slightly lower sensitivity when compared to $\mathrm{CE}$, but shows some advantages in terms of costs, ease of performance and personnel time. It is however essential to stress that clonality results should be interpreted in the context of the morphology and the clinical findings.

\section{LS 107}

\section{COMPARATIVE STUDIES OF CYTOGENETIC PROFILES OF FOLLICULAR LYMPHOMAS GRADE 3 REVEAL A SIGNIFICANTLY HIGH FREQUENCY OF BCL6 REARRANGEMENT IN FL GRADE 3B.}

\section{Moreau $\mathbf{A}^{\mathbf{1}}$, Leclair $\mathbf{F}^{\mathbf{1}}$, Talmant $\mathbf{P}^{\mathbf{2}}$, Gaillard $\mathbf{F}^{\mathbf{1}}$, Bossard $\mathbf{C}^{\mathbf{1}}$, Soubeyran $\mathbf{I}^{3}$, Rousselet $\mathbf{M C}^{4}$, Avet-Loiseau $\mathbf{H}^{2}$.}

Anatomie Pathologie A ${ }^{1}$, Hématologie Biologie ${ }^{2}$, Hotel Dieu, 44093 Nantes, France, Departement de Pathologie ${ }^{3}$, Institut Bergonié, 33076 Bordeaux, France Anatomie Pathologique ${ }^{4}$, CHU, 49933 Angers, France

Background: Follicular lymphoma (FL) grade 3 is recognized as a distinct entity in the WHO classification, and is subdivided into grade $3 \mathrm{a}$ and $3 \mathrm{~b}$. In the recent literatture, there are conflicting results regarding the biological differences between FL 3a and 3b. Aim of the study was to determine cytogenetic profiles of FL grade 3 by FISH and conventional cytogenetics in a large series. Methods : 56 nodal FL grade 3 were retrieved from the files of participating institutions and included in the study. All the cases were reviewed and graded according to the WHO classification by two pathologists. Immunohistochemistry with anti-bcl2 and $\mathrm{CD} 10$ was performed according to standard methods. The presence of the $t(14 ; 18)$ translocation and of a $3 \mathrm{q} 27 / \mathrm{bcl} 6$ rearrangement were investigated and compared using FISH analysis on whole paraffin sections. Conventional cytogenetics was also available in 10 cases. 
Results: 38 FL were classified as grade $3 \mathrm{a}$, and 18 as grade $3 \mathrm{~b}$ with $(n=3)$ or without $(n=15)$ a diffuse large B cell component. Follicular structures were present in at least $75 \%$ of the infiltrated areas. CD10 positivity was observed in 31 of $38(81.5 \%)$ FL 3 a and 11 of 17 (64.7\%) FL $3 b(p=0.009)$. The bcl2 protein was expressed in 29 of 37 $(78.4 \%)$ FL $3 a$ and in 16 of $17(94.11 \%)$ FL $3 b \quad(p=0.0001)$. Cytogenetic data were obtained in 50/56 cases, including $38 \mathrm{FL} 3 \mathrm{a}$ and 12 FL $3 b$. FISH analysis and conventional cytogenetics showed: 1 - the presence of the $\mathrm{t}(14 ; 18)$ translocation in $68.4 \%(26 / 38)$ of $\mathrm{FL}$ $3 \mathrm{a}$, including $24 \mathrm{t}(14 ; 18)+/ \mathrm{bcl} 6-$ cases, and in $66.6 \%(8 / 12)$ of FL $3 \mathrm{~b}$ $(\mathrm{p}=0.9)$; 2-the presence of a $3 \mathrm{q} 27 / \mathrm{bcl} 6$ rearrangement in $15.78 \%(6 /$ $38)$ of FL $3 a$ and in $66.6 \%(8 / 12)$ of FL $3 b(p=0.00062)$. The 3 cases of FL $3 b$ with DLBCL component were shown to harbor a $3 q 27 / b c 16$ rearrangement. The 9 double negative cases corresponded all to $\mathrm{FL}$ 3a. Most of the double positive cases corresponded to FL 3b $(4 / 6)$. Conventional cytogenetics showed a high frequency of chromosomal abnormalities involving gains and deletions in both FL grade $3 \mathrm{a}$ and $3 b(9 / 10)$. One case of FL $3 b$ was characterized by a combination of $t$ $(14 ; 18)$ translocation, bcl6 and c-myc rearrangement.

Conclusions: This study confirms that bcl6 rearrangement occurs significantly more frequently in FL $3 b \pm$ DLBCL than in FL 3a, indicating different genetic pathways in the development of the 2 types of FL grade 3 . On the other hand, there is a relatively high frequency $(66 \%)$ of $t(14 ; 18)$ translocation in our series of FL $3 b$ in comparison with the majority of reported data. These FL $3 b$ with $\mathrm{t}(14 ; 18)$ translocation were more likely to express CD10. Bcl6 rearrangement and $\mathrm{t}(14 ; 18)$ translocation were not mutually exclusive in our series. These results support the view that the separation of FL grade 3 into FL $3 \mathrm{a}$ and $3 \mathrm{~b}$ is of biological significance.

\section{LS 108}

\section{THE IMMUNE INHIBITORY RECEPTORS BTLA AND HVEM ARE DOWNREGULATED IN GERMINAL CENTER CELLS AND PREDOMINANTLY EXPRESSED IN NEOPLASTIC B-CELLS FROM SMALL LYMPHOCYTIC LYMPHOMA} B Chetaille*, H M'Hidi, Y Guillaume, ML Thibult, R Bouabdallah,
D Olive, L Xerri

Institut Paoli Calmettes, Marseille, France

Background: In order to prevent excessive activation, the immune response is tightly regulated by membrane lymphoid inhibitory receptors, including the $\mathrm{B}$ and $\mathrm{T}$ Lymphocyte Attenuator (BTLA). The interaction of BTLA with its ligand, the Herpes Virus Entry Mediator (HVEM), leads to inhibition of lymphocyte activation. Recent data suggest that the BTLA/HVEM pathway may play a role in the physiopathology of autoimmune, infectious and neoplastic diseases. So far there is no description of their expression in human normal lymphoid tissue and lymphomas.

Purpose: To characterize the expression of BTLA and HVEM in human normal and neoplastic lymphoid tissues using immunohistochemistry.

Results: In hyperplastic lymph nodes ( $\boldsymbol{n}=13$ both BTLA and HVEM are expressed in T lymphocytes within interfollicular areas and in B lymphocytes within mantle zone and marginal zone. Within germinal centers (GC), B lymphocytes are negative for both BTLA and HVEM, whereas $\mathrm{T}$ lymphocytes are BTLA positive and follicular dendritic cells are HVEM positive. In lymphomas $(\boldsymbol{n}=\boldsymbol{8 6})$, both BTLA and HVEM are strongly expressed in chronic lymphoid leukemia/small lymphocytic lymphoma (CLL/SLL, 15/15) and more weakly in marginal zone lymphomas (MZL, 8/8). Neither BTLA nor HVEM were detected at significant levels by immunohistochemistry in mantle cell lymphoma (MCL, 0/5) and follicular lymphoma (0/16).

Conclusion: The extinction of BTLA and HVEM expression in normal GC B-cells suggests that downregulation of BTLA/HVEM pathway may be involved in the process of antigen-driven B-cell activation. The preferential expression of BTLA and HVEM in SLL could be used as a help for the diagnosis of small cell lymphomas

\section{LS 109}

\section{PRE-TREATMENT OF HAEMATOLOGICAL SAMPLES REVEALS NUCLEAR EPITOPES DURING IMMUNOCYTOCHEMICAL ANALYSIS}

Göran Mattsson ${ }^{1,3}$, Susan Turner ${ }^{2}$, Margaret Jones ${ }^{1}$, Nithiya Pillai ${ }^{2}$, Teresa Marafioti ${ }^{1}$, Helle Grann Wendelboe ${ }^{3}$, Wendy Erber ${ }^{4}$, David Mason $^{1}$

1 Leukaemia Research Fund Immunodiagnostics Unit, Nuffield Department of Clinical Laboratory Sciences, ${ }^{2}$ Department of Haematology, John Radcliffe Hospital, Oxford, UK; ${ }^{3}$ R\&D Pathology, Dako A/S, Glostrup, Denmark; ${ }^{4}$ Department of Haematology, Addenbrooke's Hospital, Cambridge, UK

\section{Background:}

Rapid diagnosis of leukaemia and lymphoma in haematology today is dependent upon specialised laboratory services including immunophenotyping of haematological material. Immunophenotyping by flow cytometry allows the identification of different cell populations within a sample and several antigens may be evaluated simultaneously. Unfortunately the technology has its limitations and cannot detect different patterns of expression of intracellular antigens e.g. nucleophosmin. Therefore, additional test methods for cell markers that can be performed on haematological samples are of potential value. Immunocytochemistry is an obvious candidate; however, many antibodies fail to provide staining on fresh cell material.

Purpose of the study: To evaluate pre-treatment to improve immunocytochemistry on fresh haematological material.

Methods: Bone marrow and blood smears were fixed in a number of different fixatives before target retrieval in pressure cooker with either Citrate $(\mathrm{pH}$ 6.0) or Tris/EDTA ( $\mathrm{pH}$ 9.0). The slides were then incubated with primary antibodies (e.g. nucleophosmin, TdT, PML, Pax-5, CD20, Bcl-2). A traditional Dako APAAP visualization system was used and signals developed with Fast Red. The cell smears were counter stained with haematoxylin and evaluated by conventional light microscopy.

Results: The morphological outcome after pre-treatment depends on the fixative used and the combination of acetone/methanol/formalin performed well. Fixatives without formalin did not preserve the cellular morphology. Some markers, e.g. nucleophosmin, were completely negative on the blood smears without target retrieval. After target retrieval in the pressure cooker nucleophosmin could be detected by immunocytochemistry. Cytoplasmic and plasma membrane markers performed usually well without target retrieval.

Conclusion: Morphology preservation is dependent on the fixative. Target retrieval creates greater chances of positive staining with 
nuclear markers on cell smears. This technique provided positive immunostaining results on blood smear e.g. for nucleophosmin. The described technique opens up for further usage of immuncytochemistry on blood smears.

\section{LS 110}

\section{INTERPHASE LYMPHOMA: REPORT OF FOUR CASES FROM PERU}

Carlos Barrionuevo (*), Oswaldo Grados (**), Henry Gómez (**), Luís Mas $(* *)$, Luís Casanova (**), Sandro Casavilca $\left(^{*}\right)$, Jaime Montes (*), Natalia Valdivieso (**), Milagros Abad and Eduardo Zevallos-Giampietri $(*)$

(*) Departments of Pathology and Medicine (**) from the National Institute of Neoplastic Disease (INEN), Lima, Perú

Background: The sequential occurrence of Hodgkin lymphoma (HL) and non-Hodgkin lymphoma (NHL) (interphase lymphoma) is considered rare. More attention on this condition has been recently pointed up to understand the pathogenesis of lymphomas.

Purpose: Herein, we report four such cases from the INEN with complete clinical data, histological and immnunohistochemistry studies, and a review of the current medical literature of this topic.

Results: The age of patients ranged between 7 and 57 years at initial diagnosis of lymphoma. Two of them were diagnosed as Classical Hodgkin Lymphoma (CHL) and Nodular Lymphocyte Predominant HL. Both developed Diffuse Large B-cell Lymphoma (LCGBD) after receiving chemotherapy. The other two patients were diagnosed as Low grade Follicular Lymphoma and DLBCL and after receiving chemotherapy both developed CHL. The subsequent lymphomas were diagnosed between 2 and 38 years later.

Conclusion: The cases described above are consistent with the proposal of a single cell origin of both LH and mature NHL of B lineage. Remarkably, these cases prove not only the possibility of HL to NHL, but also the inverse situation, NHL to HL. The time of diagnosis between the two types of lymphoma is variable and can be as long as 38 years.

\section{LS 111}

\section{IGV $_{\mathrm{H}}$ GENE MUTATION STATUS AND GENOMIC IMBALANCES IN CHRONIC LYMPHOCYTIC LEUKAEMIA WITH INCREASED PROLYMPHOCYTES (CLL/PL)}

*Zsófia Balogh, Lilla Reiniger, Linda Deák, Csaba Bödör, Judit Csomor, Ágota Szepesi, Éva Gagyi, László Kopper and András Matolcsy

1st Department of Pathology and Experimental Cancer Research, Faculty of Medicine, Semmelweis University, Budapest, Hungary

Chronic lymphocytic leukaemia (CLL) with increased prolymphocytes (CLL/PL) has been defined by the World Health Organization (WHO) classification and considered as a progressive and clinically aggressive variant of CLL. To further characterize the biological features of this disease, we performed IgVH gene mutational status,
FISH and high resolution comparative genomic hybridization (HRCGH) analysis in 17 cases of CLL/PL. All CLL/PL utilized members of VH1, VH3 and VH4 families, with the highest prevalence of the VH1-69 gene. In all but one cases analyzed, the VH genes were unmutated. The FISH and HR-CGH analyses showed frequent occurrence of trisomy 12, del(11)(q23), del(17)(p13) and genetic imbalances, but recurrent genetic lesion characteristic for CLL/PL was not found. The follow-up HR-CGH analysis of two cases showed that increase of prolymphocytes in the course of CLL or CLL/PL are associated with clonal evolution and selection of the tumour clone.

In conclusion, this study suggests that CLL/PL is a relatively homogeneous disease regarding $\mathrm{VH}$ gene mutation, but heterogeneous regarding genetic lesions. The heterogeneity and high number of genomic imbalances found in CLL/PL suggest that a genome-wide instability of the neoplastic cells may play a role in the development of the disease.

\section{LS 112}

\section{DIAGNOSIS AND DIFFERENTIAL DIAGNOSIS OF SPORADIC BURKITT'S LYMPHOMA AND DIFFUSE LARGE B-CELL LYMPHOMA}

Cuiling Liu ${ }^{1}$, Yuanjie Huang ${ }^{1}$, Liping Gong ${ }^{2}$, Min Li ${ }^{1}$, Xin Huang ${ }^{1}$, Zifen $\mathrm{Gao}^{1 *}$

1 Department of Pathology, Peking University Health Science Center, Beijing 100083, China

2 Department of Pathology, Capital Medical University, Beijing 100069, China

*Corresponding author: Zifen Gao (E-mail: wjshgao@bjmu.edu.cn)

BL and DLBCL share some overlapping morphological features. To investigate the pathological features and their differential diagnosis of sporadic Burkitt's lymphoma (BL) and diffuse large B-cell lymphoma (DLBCL), 20 cases of sporadic BL and 20 cases of DLBCL were retrospectively studied by analyzing their light microscopy features, immunohistochemical expression, EBV infection detected by in stiu hybridization, chromosomal breakage of c-myc and/or IgH genes by interphase fluorescence in stiu hybridization (FISH), and their clinical manifestation. Studies showed that all the 20 cases of sporadic BL occurred in children (younger than 14 year-old), while all the DLBCL patients were older than 15 year-old. Microscopically, the mediumsized tumor cells were monomorphic and proliferated in a diffuse pattern in both BL and DLBCL cases. But comparing with DLBCL, BL showed marked "starry-sky" with numerous karyorrhectic debris, and mitotic figures were more frequently seen in BL. Immunohistochemically, the tumor cells of both BL and DLBCL were positive for CD20 and negative for TdT, CD99, MPO. CD10 were positive in $100 \%$ of BL cases, while positive in $43 \%$ of DLBCL cases. No EBV infection was found by in situ hybridization in all the detected cases. Interphase FISH analysis detected c-myc gene breakage or amplification and/or IgH/c-myc gene breakage in all detected BL cases, but none in DLBCL cases. So, it seems that molecular and immunohistochemical analysis plays a vital role on their correct diagnosis and differential diagnosis. EBV infection is not related with sporadic BL and DLBCL in China. 


\section{LS 113}

\section{EXPRESSION OF CD43 IS AN IMPORTANT PROGNOSTIC FACTOR IN A DIFFUSE LARGE B-CELL LYMPHOMA}

*I. Ilic ${ }^{1}$, Z. Mitrovic ${ }^{2}$, I. Aurer ${ }^{2}$, S. Basic-Kinda ${ }^{2}$, I. Radman ${ }^{2}$, R.

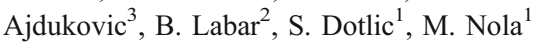

${ }^{1}$ Pathology and ${ }^{2}$ Hematology, University Hospital Center and Medical School, Zagreb, Croatia; ${ }^{3}$ Hematology, University Hospital Dubrava, Zagreb, Croatia

Background. CD43 is a membrane protein expressed on all hematopoetic cells except resting B-cells and erythrocytes with a postulated role in adhesion, T-cell activation and apoptosis. CD43 is expressed in about $20 \%$ of diffuse large B-cell lymphoma (DLBCL) but its prognostic significance in DLBCL is still unknown.

Purpose of the study. To define the prognostic significance of CD43 on DLBCL.

Methods. With a use of a standard immunohistochemistry we analyzed the protein expression of CD43 and its prognostic significance in 101 patients with DLBCL consecutively collected from our archive. All patients were treated with anthracycline-based chemotherapy or with antracycline-based therapy combined with rituximab.

Results. CD43 was expressed in 25/101 patients (25\%). The median follow-up of survivors was 36 months (range 1-177 months). CD43+ patients had significantly lower complete response rates $(56 \%$ vs. $84 \%, \mathrm{p}<0.001)$, inferior event-free survival (EFS) $(26 \%$ vs. $66 \%$ at 2 years, $\mathrm{p}<0.001)$ and overall survival (OS) ( $44 \%$ vs. $71 \%$ at 2 years, $\mathrm{p}=0.001)$ than CD43- patients. The prognostic impact of CD43 was not affected by rituximab treatment. CD43 expression was not related to IPI score, stage, LDH, age, performance status or GCB vs. nonGCB type as determined by immunohistochemistry.

Conclusion. The expression of CD43 in patients with DLBCL identifies a subgroup of patients with significantly inferior response rates, EFS and OS. This effect is independent of the IPI score or rituximab treatment. The data presented suggest that expression of CD43 is an independent prognostic factor in DLBCL.

\section{LS 114}

\section{GERMINAL CENTERS CONTAINING NUMEROUS EBER-POSITIVE CELLS IN RESIDUAL GERMINAL CENTERS IN HODGKIN'S LYMPHOMA}

Martin P, Gomez-Lozano N, Lozano M, Provencio M, Soutar D, Salas C, Montes S, Bellas C.

Accumulated evidence suggests that EBV (Epstein Barr virus) has an etiological role in the pathogenesis of $\mathrm{cHL}$, as was initially suspected on the basis of epidemiological and serological findings.

The neoplastic cells of Hodgkin tumours are clonal and derived from GC-B cell (germinal center) because they carry somatic mutations of their rearranged $\mathrm{IgVH}$ genes. These findings strongly suggest that EBV infected precursor cells should be found in GCs. In contrast to this assumption, GCs are generally believed to be devoid of EBVinfected cells. Although EBER-positive lymphoid cells have been found in GC of hyperplastic tonsils from children living in developing regions (with high frequency of EBV-associated lymphomas), concomitant infection in residual GC of cHL has not been reported. In this study, we describe 4 cases of cHL EBV associated, with presence of EBV infection in B-cells of GC.

Material and Methods: Diagnosis of cHL was made on the basis of histology and immunohistology examination, according with the WHO classification. DNA was isolated from paraffin-embedded tissue. Freshly frozen material for DNA extraction was available in 2 cases. Molecular studies were done using PCR in DNA of whole tissue and microdissected cells, to identify the specific cellular location of the viral sequences present in different cellular populations (HRS cells and GC-cells). EBV typing was performed by amplifying a strain-specific sequence in the nuclear antigen 3C (EBNA-3C) and also we tested the $30 \mathrm{bp}$ deletion in the C-terminal end of the LMP-1 gene.

Results: All specimens of cHL had reactive residual GC. EBERs were detected in the nuclei of the malignant cells (HRS), and LMP-1 stained the cell membrane and the Golgi region. Moreover the majority of the GC cells expressed EBERs, and LMP-1 was negative. Molecular studies showed the same strain of EBV in microdissected GC cells and HRS cells.

Conclusions: Our results show that latently EBV-infected cells participate in $\mathrm{GC}$ reaction in cases of $\mathrm{cHL}$ with down regulation of LMP-1 expression.

These findings demonstrate in vivo a pool of EBV-infected cells in the compartment from which Hodgkin's lymphoma are thought to arise.

\section{$\underline{\text { LS } 115}$}

\section{A PATHOLOGIC STUDY OF HODGKIN LYMPHOMA IN MOROCCO AND ITS ASSOCIATION WITH EPSTEIN-BARR VIRUS}

*L. Jabri, A. Belbachir, A. Benkirane, M. Quachouh, A. Quessar, A. Madani, S. Zamiati

Pathology Department;

Hematology department

Ibn Rochd University Hospital, Casablanca, Morocco

Background: The epidemiological data of Hodgkin Lymphoma (HL) shows a high incidence in children and a predominance of Mixed cellularity in developing countries. The association of Epstein- Barr virus (EBV) and HL is well known and correlates with the histologic subtype, age of presentation and geographic location. Objectives: the aim of this study is to determine the epidemiological and histopathological profil of Moroccan Hodgkin Lymphoma and to evaluate the incidence of EBV in this population.

Methods: We evaluated the clinical and morphological data of 324 patients diagnosed between January 2000 et December 2005. Immunohistochemical features were available for 278 patients and 234 were analyzed for Epstein-Barr virus (EBV) using latent membrane protein (LMP1).

Results: There were $53 \%$ males and $47 \%$ females, with a mean age of 32 years. The peak of incidence was seen in the 15-39 years age group $(53 \%) .16 \%$ of the cases were seen in children under fifteen. Nodular sclerosis was the most prevalent subtype ( $60 \%$ versus $24 \%$ for mixed cellularity. Expression of CD30 was detected in 255 of 278 cases (92\%), CD15 was positive in 2280f $278(82 \%), \mathrm{Bcl} 2$ was detected in 
$55 \%$. LMP1 was seen in 124 of 234 cases $(53 \%)$ : 74 of $155(48 \%)$ with nodular sclerosis; 42 of $56(75 \%)$ with mixed cellularity. There was no difference in LMP1 positivity between localised clinical stages and dvanced clinical stages. LMP1 positivity was higher in children and older adults than in adults aged between 15-50 years.

Conclusions: HL in Morocco showed a high incidence of nodular sclerosis subtype and a high prevalence of EBV. EBV detection showed correlation with mixed cellularity subtype and age under Fifteen. LMP1 was more frequently seen in children and older adults, suggesting a different pathophysiology of HL among different age groups

\section{LS 116}

\section{APPROACH TO IDENTIFY THE GENES WHICH ARE INVOLVED IN THE EXTINCTION OF THE B-CELL PROGRAM IN CLASSICAL HODGKIN LYMPHOMA BY EPIGENETIC REPROGRAMMING OF NEOPLASTIC B-CELLS}

Harald Stein ${ }^{1}$, Anke Ehlers ${ }^{1}$, Elisabeth Oker $^{1}$, Stefan Bentink ${ }^{2}$, Dido Lenze ${ }^{1}$, Michael Hummel ${ }^{1}$.

\footnotetext{
${ }^{1}$ Institut für Pathologie, Charité - Berlin, Campus Benjamin Franklin; ${ }^{2}$ Institut für funktionelle Genomik, Computational Diagnostics Group, Regensburg
}

Background: The tumour cells of aggressive B-cell lymphoma (aBCL) consistently show an immunophenotype of mature B cells with expression of all B-cell typical and transcription factor genes. In contrast, the B-cell derived tumour (HRS) cells of classic Hodgkin lymphoma (CHL) have almost completely lost their B-cell identity. In addition, HRS cells acquired several B-cell lineage inappropriate antigens. The purpose of our study is to investigate a) whether epigenetic mechanisms play a role in the development of the unique phenotype of HRS cells, b) whether this approach aids to answer the question whether the typical CHL gene expression program is caused by a down- or up-regulation of genes and c) whether the genes can be identified which are involved in the trans-differentiation of B-cells to HRS cells.

Material and methods: CHL and aBCL lines were treated with DNA-demethylation (5-aza-dC) and histone-acetylation (TSA) reagents. Treated and untreated cell lines were analysed by Affymetrix GeneChips. Numerous up- and down-regulated genes were verified by quantitative RT-PCR and Western blot analysis. Chromatin-immunoprecipitation was carried out to determine the epigenetic modifications in the promoter region of the corresponding genes.

Results: The treatment of CHL lines with 5-aza-dC and TSA had no significant effects on the reactivation of the B-cell expression program. Instead, the treatment of aBCL lines resulted in a complete loss of their B-cell phenotype and - in parallel - to an up-regulation of Hodgkin-characteristic genes. Our data clearly demonstrate that DNAdemethylation and histone-acetylation is able to re-program B cells into cells with a Hodgkin-like phenotype. In contrast, no re-activation of B-cell typical genes or suppression of B-cell inappropriate genes was observed in HRS cells.

Conclusions: These findings suggest that in CHL the silencing of the B-cell expression program is not due to a down-regulation but rather due to an up-regulation of certain genes and simultaneous demonstrate the plasticity of B-cells.

\section{LS 117}

\section{ALK POSITIVE (+) DIFFUSE LARGE B-CELL LYMPHOMA (DLBCL) OF BONE - CASE REPORT}

*L. Jabri 1, A.Benkirane 1, M. Karkouri 1, S. Benayad 1, A. Madani 2, A. Quessar2, S. Zamiati 1

1Pathology departmemt

2Heamatology depertment

University Hospital Ibn Rochd, Casablanca, Morocco

Background: Anaplastic lymphoma kinase ${ }^{+}\left(\mathrm{Alk}^{+}\right)$DLBCL is a rare subtype of B-cell non-Hodgkin's lymphoma (NHL), originally described in 1997 by Delsol et al. It has a plasmoblastic morphology and is characterized by positive CD138, and negative CD20, and in comparison to anaplastic large cell lymphoma it is also positive to epithelial membrane antigen (EMA), but negative to CD30. The Alk protein has a unique cytoplasmatic granular pattern and the associated karyotype may be $\mathrm{t}(2 ; 5)$ but more often $\mathrm{t}(2 ; 17)(\mathrm{p} 23 ; \mathrm{q} 23)$ fusing Alk to CLTC gene.

Objective: To describe a case of an extranodal Alk (+) DLBCL, and review the literature.

Case report: This is the report of a case of a 56-years old patient, male, who presented with lombar pain which he was experiencing from 4 months in a context of a bad general health status (?). Clinically, there was no peripheral adenomegaly. Spine plain radiographs showed a vertebral lysis of L4-L5 and there was a pancytopenia on the hemogramm. Vertebral biopsy showed diffuse large cells proliferation, with vesicular and round nuclei and prominent nucleoli, remaining sometimes of plasmablastic pattern. On immunohistochemistry, the tumoral celles expressed EMA, CD45, CD138. CD79a, CD20, Kappa, Lambda. CD30, CD15 and KL1 were negative. Tumoral cells show a cytoplasmic granular positivity with ALK1. Then the diagnosis of ALK1 positive diffuse large B cell lymphoma was made. The bone marrow biopsy of this patient was positive, invade by the same proliferation.

The patient died within the first line of a mutiagent chemotherapy.

Conclusion: Only very few cases of Alk(+) B-cell lymphoma were reported in the literature. Outcome on the different protocols and modalities was grave. Effort is needed to investigate this rare aggressive lymphoma for a better diagnostics, treatment and outcome in the future.

\section{$\underline{\text { LS } 118}$}

CD34 $^{+}$CORD BLOOD CELL-TRANSPLANTED MICE AS A MODEL FOR EPSTEIN-BARR VIRUS INFECTION OF THE HUMAN IMMUNE SYSTEM. A MORPHOLOGICAL, IMMUNOPHENOTYPICAL AND MOLECULAR STUDY.

*Mario Cocco, Cristiana Bellan, Roxane Tussiwand, Davide Corti, Elisabetta Traggiai, Stefano Lazzi, Susanna Mannucci, Lucio Bronz, Nazzareno Palummo, Piero Tosi, Antonio Lanzavecchia, Markus G. Manz, Lorenzo Leoncini.

Department of Human Pathology and Oncology, Division of Pathological Anatomy, University of Siena, Siena, Tuscany, 53100, Italy.

Institute for Research in Biomedicine, Bellinzona, CH-6500, Switzerland. Ospedale San Giovanni, Bellinzona, CH-6500, Switzerland. 
Recent studies suggest that Epstein-Barr virus (EBV) infects naïve B cells, driving them to differentiate into resting memory B cells via the germinal center reaction. This has been inferred from parallels with the biology of normal $\mathrm{B}$ cells but has never been proved experimentally. Rag2 $2^{-/-} \gamma_{\mathrm{c}}^{-/-}$mice, transplanted with human $\mathrm{CD} 34^{+}$cord-blood cells as newborns, were recently shown to develop human B, T, and dendritic cells, constituting lymphoid organs in situ. Accordingly, we reasoned that this model would be useful to better define the strategy of EBV infection of human B cells in vivo and to compare this with different conditions of EBV infection in humans.

Fourteen mice were constituted with $\mathrm{CD} 34^{+}$cord blood cells obtained from four different donors. Among them, twelve were infected by intraperitoneal injection with of EBV B95-8 supernatant and were subsequently sacrificed at different time points. Two uninfected mice were used as uninfected controls. In the four groups of mice tested at different interval after infection two outcomes of experimental infection have been observed. One characterized by follicular hyperplasia with florid germinal centers and one characterized by nodular and diffuse lymphoid proliferation. Indeed, the only difference found among cases showing different morphology, immunophenotype and molecular characteristics was the type of T cell infiltration. Our results suggests that the outcome of EBV infection in $\operatorname{Rag} 2^{-/-} \gamma_{\mathrm{c}}^{-/-}$ cord blood reconstituted mice does not depend on the source of CD $34^{+}$cord blood cells used and the level of pre-infection human peripheral blood engraftment, but rather on the quality of the immune response against infected cells. Furthermore they reveal different strategies of EBV infection in B cells, possibly resulting from variations in the host immune response.

\section{LS 119}

\section{EXPRESSION OF CANNABINOID RECEPTORS TYPE 1 AND TYPE 2 IN NON HODGKIN LYMPHOMA: GROWTH INHIBITION BY RECEPTOR ACTIVATION}

Gustafsson, K., Wang, X., Severa, D., Eriksson, M., Kimby, E., Merup, M., Christensson, B., Flygare, J. and Sander, B.*

Karolinska Institutet and Karolinska University Hospital Huddinge, Stockholm, Sweden

Background: Cannabinoids exert anti-proliferative effects in various types of cancer and in mantle cell lymphoma (MCL).

Purpose of the study : To investigate the expression of cannabinoid receptors type 1 and type 2 (CB1 and CB2) in non Hodgkin lymphoma of the $\mathrm{B}$ cell type and to evaluate the functional response of receptor targeting. Summarized description of the results : MCL uniformely expresses higher levels of $\mathrm{CB} 1$ and $\mathrm{CB} 2$ than reactive lymphoid tissue. In indolent and aggressive lymphomas (small lymphocytic lymphoma, follicular lymphoma, marginal zone lymphoma, Burkitt lymphoma, diffuse large B cell lymphoma) the levels of cannabinoid receptor mRNA expression was highly variable, ranging from 0.1-224 times the expression in reactive lymph nodes. By immunohistochemistry $\mathrm{CB} 1$ expression was detected in the tumour cells. The stable analogue of endogenous cannabinoids, methanandamide, induced cell death in MCL and CLL but not in Burkitt lymphoma. In vivo treatment with methandamide caused a significant reduction of tumor size and mitotic index in mice xenografted with MCL.

Conclusion: Therapies using cannabinoid receptor ligands could be part of a future therapy for malignant lymphoma

\section{LS 120}

\section{NASAL NK/T CELL LYMPHOMA REPORT OF 13 CASES}

*L. Jabri 1, F.Marnissi 1, A. Benkirane1, M. Rachid 2, A. Quessar 2, S. Zamiati 1

1 Department of pathology

2 Department of Hematology

University Hospital Ibn Rochd, Casablanca, Morocco

Nasal NK/T cell lymphoma occurs in the nasal cavity and nasopharynx, with variable regional extension. It is called "NK/T" instead of "NK" because there is still some uncertainty concerning its exact lineage. This lymphoma is rare in western populations and much more prevalent in some Asian and Latin American countries. There is a very strong association of this lymphoma with Epstein-Barr (EBV) (>95\%) and this later is considered to be the etiologic agent.

We studied 13 cases of Nasal NK/T cell lymphoma collected in our Department between 2000 and 2005 to characterize their clinicopathologic features and to study the prevalence of EBV using immunohistochemical detection of latent membrane protein LMP1. Nine patients were men and 4 were women. Their age ranged from 17 to 70 years old with a median age of 40 years. All cases displayed an immunophenotypic profile of CD3 (epsilon) +, CD20-, except three cases that were CD3 (epsilon) negative and among them two cases were CD43 positive. Granzyme B was positive in 10 cases. LMP1 was positive in 5 cases.

Our sample is certainly small but our results are in keep with previous studies published. The causality of EBV in nasal NK/T cell lymphoma is highly suspected.

\section{LS 121}

\section{AN AGGRESSIVE NASOPHARYMGEAL LYMPHOMA WITH PLASMABLASTIC FEATURES IN AN IMMUNOCOMPETENT PATIENT: Case report}

*L. Jabri 1, F.Marnissi 1, A. Benkirane1 , A. Madani 2 , A. Quessar 2, S. Zamiati 1

1 Department of pathology

2 Department of Hematology

Lymphomas with plasmablastic differentiation are a heterogeneous group of neoplasm. Plasmablastic morphology and phenotype can be identified in dedifferentiated (plasmablastic) myelomas / plasmacytomas, in Plasmablastic lymphoma (PBL) a variant of diffuse large B-cell lymphoma (DLBCL) and in other aggressive DLBCLs. These tumours exhibit overlapping features leading to difficulties in differential diagnosis. We report a case illustrating this difficulty diagnostic dilemma. A 56 year-old woman presented epitasis due to an enlarging nasopharyngeal mass. Biopsy showed a tumour composed of sheets of large cells with a cohesive pattern, a large nuclei, moderately coarse chromatin, and prominent nucleoli. Many of the cells had abundant, eccentric cytoplasm, suggesting plasma-cell differentiation. The cells were positive for EMA and CD138 and were negative for cytokeratine, CD45, CD20, CD3 and 
immunoglobulin light chains lambda and kappa. The tumor proliferation rate was shown to be approximately $50 \%$.

The diagnosis proposed was plasmablastic lymphoma or an extramedullary Plasmablastic Tumor Secondary to Plasma Cell Neoplasm. Correlation with clinical feature disclosed a history of kappa light chain myeloma thus diagnosis of plasmablastic myeloma was retained.

Plasmablastic lymphoma is a distinctive type of diffuse large B-cell lymphoma found predominantly in the oral cavity mainly of HIVpositive individuals. The cellular morphology resembles immunoblastic DLBCL, the immunophenotypic features show the plasma cell differentiation.

PBLs are morphologically and phenotypically similar to extramedullary large cell transformation of plasma cell neoplasms. The distinction between these neoplasms may be impossible without clinical correlation.

Because these lymphomas don't express the more common lymphoid and/or B-cell markers, they may be mistaken for a poorly differentiated carcinoma.

\section{LS 122}

GENOMIC PROFILING IN SPLENIC MARGINAL ZONE LYMPHOMA: DELETIONS ON 7Q TARGET 7Q32

Watkins $\mathrm{AJ}^{*^{1}}$, Johnson $\mathrm{N}^{1}$, Ye $\mathrm{HT}^{1}$, de Leval $\mathrm{L}^{2}$, Huang $\mathrm{Y}^{1}$, Chanudet $\mathrm{E}^{1}$, Hamoudi $\mathrm{R}^{1}$, Liu $\mathrm{H}^{1}$, Isaacson $\mathrm{PG}^{3}$, Attygalle $\mathrm{AD}^{4}$, Wotherspoon $\mathrm{A}^{4}$, Du $\mathrm{MQ}^{1}$

${ }^{1}$ Department of Pathology, University of Cambridge, Cambridge, UK; ${ }^{2}$ Department of Pathology, CHU Sart-Tilman, Liège, Belgium; ${ }^{3}$ Department of Histopathology, University College London Hospitals, London, UK; ${ }^{4}$ Department of Histopathology, Royal Marsden Hospital, London, UK.

Background: Splenic marginal zone lymphoma (SMZL) is a poorly characterised low grade B-cell lymphoma with highly heterogeneous clinical and histological presentation. Clinical risk stratification and histological diagnosis of SMZL, in particular its differential diagnosis from other primary or secondary splenic lymphomas, remain a challenge. This is largely due to a lack of specific phenotypic as well as genetic markers. In order to characterise the genetic makeup of this enigmatic lymphoma, we investigated the genomic profiles of a series of well characterised cases using array comparative hybridisation (aCGH).

Methods: A total of 26 cases of SMZL (18 frozen tissue, 8 formalin fixed tissue) were successsfully investigated using an in house $1 \mathrm{Mb}$ resolution aCGH platform. Recurrent copy number changes in chromosome 7 were further studied by chromosome 7 tile-path aCGH. Somatic hypermutation in the rearranged IGH genes was examined by PCR and sequencing.

Results: Array $\mathrm{CGH}$ at $1 \mathrm{Mb}$ resolution revealed recurrent gain of $3 \mathrm{q}$ (4/26=15\%, including 2 cases showing trisomy 3$), 8 \mathrm{q}(5 / 26=19 \%)$, 9q33.3-34.3 (5/26=19\%, including 1 showing a focal amplification), $12 \mathrm{q}(3 / 26=12 \%$, including 1 with trisomy 12$), 18(2 / 26=7 \%)$, and recurrent loss of $7 \mathrm{q}(10 / 26=38 \%)$ and $8 \mathrm{p}(3 / 26=12 \%)$. Chromosome 7 tile-path aCGH analysis of the cases with $7 \mathrm{q}$ deletion showed an $11 \mathrm{Mb}$ region spanning $119 \mathrm{Mb}-130 \mathrm{Mb}$ at $7 \mathrm{q} 31.32-32.3$ as the minimal common deleted region (MDR). Bioinformatic search of this MDR showed no recognised tumour suppressor genes, but nonethe- less revealed several microRNAs that may be potentially relevant to the pathogenesis of this lymphoma. Somatic hypermutation of the rearranged IGH gene was seen in 23/35 (66\%) SMZLs investigated. There was no significant correlation among the CGH findings, IGH somatic mutation, clinical and histological parameters.

Conclusion: Deletion of 7q, with an $11 \mathrm{Mb}$ MDR at 7q31.32-32.3, is a frequent event in SMZL. We are in the process of investigating further the incidence and extent of this deletion in a large cohort of both primary and secondary splenic lymphomas by interphase fluorescence in situ hybridisation.

\section{$\underline{\text { LS } 123}$}

\section{GENOMIC PROFILES OF ENTEROPATHY-TYPE T-CELL LYMPHOMA AND PRIMARY INTESTINAL NK/T CELL LYMPHOMA}

*Joaquim Carreras ${ }^{1}$, Shih-Sung Chuang ${ }^{2}$, Anne Lavergne-Slove, ${ }^{3}$ Rifat Hamoudi ${ }^{1}$, Estelle Chanudet ${ }^{1}$, Hongxiang Liu ${ }^{1}$, Lakshmi Venkatraman ${ }^{4}$, Ahmet Dogan ${ }^{5}$, Peter G. Isaacson ${ }^{6}$, Jeremy Woodward ${ }^{7}$, Nadine Cerf-Bensussan ${ }^{8}$, Ming-Qing $\mathrm{Du}^{1}$.

${ }^{1}$ Department of Pathology, Cambridge University, UK; ${ }^{2}$ Department of Pathology, Chi-Mei Medical Center, Tainan, Taiwan; ${ }^{3}$ Anatomie Pathologique, Hôpital Lariboisière, Paris, France; ${ }^{4}$ Department of Histopathology, Royal Victoria Hospital, Belfast, UK; ${ }^{5}$ Department of Pathology, Mayo Clinic, Rochester USA; ${ }^{6}$ Department of Histopathology, University College London, London, UK; ${ }^{7}$ Department of Gastroenterology, Addenbrooke's NHS Trust, Cambridge, UK; ${ }^{8}$ INSERM U793, Paris, France.

Background: Enteropathy-type T-cell lymphoma (ETL) and intestinal NK/T-cell lymphoma (NK/TCL) of nasal-type are very aggressive lymphomas with poor prognosis. ETL is assumed to originate from intraepithelial $\mathrm{T}$ cells (IEL) of the small intestine in patients with celiac disease and it is often preceded by type II refractory celiac disease (RCD), which is characterised by immunophenotypically aberrant and monoclonal IELs. Intestinal NK/TCL is believed to derive from NK cells or cytotoxic T lymphocytes and is invariably associated with EBV infection. The molecular genetics underlying the development of these aggressive intestinal lymphomas is unknown.

Methods: DNA samples extracted from IEL cell lines of $3 \mathrm{RCD}$, the tumour cells microdissected from $27 \mathrm{ETL}$ and $17 \mathrm{NK} / \mathrm{TCL}$ were successfully profiled for genomic copy number alterations using an in house $1 \mathrm{Mb}$ resolution array comparative genomic hybridization $(\mathrm{aCGH})$. Recurrent changes in chromosomes 1, 6, 7 and 9 were further investigated by corresponding chromosome tilling path array in selected samples.

Results: RCD IEL cell lines showed gains of 1q21.1-25.5 (2/3), 6 p22.3-25.3 (2/3) and $+21(1 / 3)$; and deletions of 1p31 (1/3), 6q16.1$22.1(1 / 3), 10 \mathrm{q} 24.32(1 / 3)$ and 13q21.31-34 (1/3). ETL displayed recurrent gain of 9q34.11-34.3 (44\%), 1p36.32 (44\%), 7p22.3 (41\%), $13 \mathrm{q} 34$ (33\%), 4p16.3 (30\%), 7q36.3 (30\%), 2q37.1 (19\%) 1q22 $(15 \%)$ and $18 \mathrm{q} 23(15 \%)$. NK/TCL was characterized by gains of 9q34.11-34.3 (27\%), 1p36 (9\%), 7p22.3 (9\%), 13q34 (9\%) and losses of $6 \mathrm{q}(32 \%), 9 \mathrm{p}(23 \%), 7 \mathrm{p}(18 \%)$ and $10 \mathrm{q}(9 \%)$.

Conclusion: There are common and distinct genomic copy number changes among RCD-II, ETL and intestinal NK/TCL. Identification of these recurrent genomic alterations provides a basis to further 
investigate their role in multistage development of ETL and their value in RCD diagnosis.

\section{LS 124}

\section{PRESENCE OF MONOCLONAL T-CELL POPULATIONS IN B-CELL POST TRANSPLANT LYMPHOPROLIFERATIVE DISORDERS.}

* Hazem AH Ibrahim, Lia Menasce, and Kikkeri N Naresh.

Departments of Histopathology, Hammersmith Hospital and Imperial College London, UK \& Christie Hospital, Manchester,UK.

Background: Post-transplant lymphoproliferative disorders (PTLDs) arise as a result of immune suppression and loss of immune surveillance. Most cases are B-cell PTLDs. Rare cases of T-cell PTLDs are described. Purpose of the study: We hypothesised that the lack of immune surveillance in B-PTLD should also be affecting the T-cells, though they may not be histologically identifiable. We investigated 18 cases of well established B-PTLD for presence of clonal T-cell populations. Description of results: DNA was extracted from paraffin sections of 18 cases of B-PTLD, 10 cases of diffuse large B-cell lymphoma (DLBCL) and 5 reactive lymph nodes (RLN). Clonality assessment was carried out by polymerase chain reaction (PCR) using T-cell receptor gamma chain gene primers and the amplicons were subjected to heteroduplex analysis. Six of 18 B-PTLDs showed evidence of a monoclonal T-cell expansion. One of 18 PTLDs and one of the 10 DLBCLs showed presence of a dominant amplicon in the background of polyclonal/oligoclonal T-cell expansion suggesting a minor clone. Rest of PTLDs and DLBCLs and all RLNs did not show evidence of monoclonal T-cell expansion. Among PTLD cases, CD3, CD4 and CD8 positive cells were counted and expressed as cells $/ \mathrm{mm}^{2}$ on tissue microarry sections. All cases of B-PTLD with a monoclonal T-cell population showed dominance of CD8 positive cells, and 4/6 of them had a CD4:CD8 ratio of $<0.2$. None of the cases without monoclonal T-cell expansion had a ratio $<0.2$. Review of histological features in one of the cases suggested features that were morphologically compatible with a co-existing T-PTLD of small cell type.

Conclusion: Monoclonal expansion of T-cell population occurs frequently in the post-transplant lymphoproliferative setting. These clonal T-cell populations co-exist with monoclonal B-cell population in B-PTLD.

\section{LS 125}

\section{B-CELL POST TRANSPLANT LYMPHOPROLIFERATIVE (B-PTLD) DISORDERS ARE NOT ASSOCIATED WITH THE CHARACTERISTIC LYMPHOMA-ASSOCIATED CHROMOSOMAL TRANSLOCATIONS.}

\footnotetext{
* Hazem AH Ibrahim, Lia Menasce, Mark Bower, and Kikkeri N Naresh.
}

Departments of Histopathology, Hammersmith Hospital and Imperial College London, UK \& Christie Hospital, Manchester,UK.

Background: PTLDs are group of lymphoproliferative disorders that develop in the background of immune suppression. They are often associated with Epstein Barr Virus (EBV) and thought to be associated with accumulation of genetic and epigentic changes. It has been reported that the characteristic lymphoma-associated chromosomal translocations are rare among PTLD.

Purpose of the study: We intended to confirm the previous observation of lack of classical lymphoma-associated chromosomal translocations in PTLDs and to widen the translocatoin loci investigated.

Description of the results: We carried out fluorescent in-situ hybridization (FISH) on tissue microarray sections in 15 cases of B-PTLD, 14 HIV-associated B-cell lymphomas (7 Burkitt Lymphoma, 7 Diffuse large B-cell lymphoma (DLBCL)), and 40 cases of immunocompetent (IC)-DLBCLs, using split-apart probes for BCL2, BCL3, BCL6, CMYC, PAX5, and IGH loci. One out of 15 B-PTLD showed both CMYC and IGH translocations. The case had morphology of monomorphic B-PTLD (DLBCL, plasmablastic subtype). Fourteen out of 15 PTLDs were negative for BCL2, BCL3, BCL6, CMYC, PAX5, and IGH translocations. On the other hand, 6/7 HIV-BL were positive for CMYC and IGH translocations. All HIV-DLBCL were negative for all translocations investigated. Among IC-DLBCL, 12/39, 7/40, 4/38, 1/36, and 1/36 were positive for IGH, BCL6, BCL2, CMYC and BCL3 translocations respectively. All the translocations were mutually exclusive. One case was positive for BCL6 translocation and negative for IGH suggesting a different partner chromosome. All cases were negative for PAX5 translocation.

Conclusion: Characteristic lymphoma-associated chromosomal translocations are very rare among PTLDs and mechanisms other than chromosomal translocations, are more likely to be involved in the pathogenesis of this disorder.

\section{$\underline{\text { LS } 126}$}

FOLLICULAR LYMPHOMA PRESENTING WITH HIGH GRADE TRANSFORMATION AND DISCORDANT RESULTS OBTAINED BY COMMERCIAL FUSION AND BREAK APART FISH PROBES

Tomas Balharek ${ }^{1}$, Martina Barthova ${ }^{1}$, Marcela Mrhalova ${ }^{2}$, Peter Szepe ${ }^{1}$, Zora Lasabova $^{3}$, Tatiana Burjanivova ${ }^{1}$, Juraj Marcinek ${ }^{1}$, Lukas Plank ${ }^{1}$

${ }^{1}$ Department of Pathology and National Consultation Centre of Haematopathology, Comenius University, Jessenius Faculty of Medicine and Faculty Hospital, Martin, Slovak Republic

${ }^{2}$ Department of Pathology and Molecular Medicine, Charles University, 2nd Medical School and Faculty Hospital in Motol, Prague, Czech Republic ${ }^{3}$ Department of Molecular Biology, Comenius University, Jessenius Faculty of Medicine, Martin, Slovak Republic

Background: Rearrangements involving BCL2 gene characteristic for follicular lymphomas (FL) may be detected by PCR and FISH. FISH may be performed using fusion or break apart/split probes. Break apart/split probes are reported to be more sensitive with minimized false positivity. In recently examined FL we noticed some discordancies of results obtained by commercial FISH probes detecting BCL2 rearrangement.

Methods: Diagnosis FL grade 1 was established according to typical morphology and immunophenotype (CD20+, CD10+, bcl-2+, bcl- $6+)$ in the biopsy of skin tumour. Two months later patient presented with gastric tumour which was classified as DLBCL, centroblastic variant with GCB phenotype (CD10+/-, bcl-6+/-, MUM-1-, bcl-2+). The blastic transformation of previously diagnosed low grade FL was supposed. We 
examined paraffin-embedded tissue sections from both materials for BCL2 rearrangement and IgH/BCL2 translocation by FISH and PCR. Three different commercial FISH probes were used: 1.) LSI IGH/BCL2 dual color dual fusion probe (Vysis), 2.) LSI BCL2 dual color break apart rearrangement probe (Vysis) and 3.) BCL2 split signal probe (Dako). PCR was performed according to BIOMED-2 protocol using primers covering MBR and mor regions.

Results: Both materials were negative for IgH/BCL2 translocation examined by BIOMED-2 PCR. FISH demonstrated that both tumours carry identical genetic abnormality and are clonally related. Vysis IGH/ BCL2 fusion probe showed abnormal FISH pattern 2O1G1F, so that the rearrangement of BCL2 accompanied with deletion of IgH gene was supposed. In contrast, Vysis BCL2 break apart probe showed only 2 fusion signals corresponding to 2 virtually non-rearranged BCL2 genes. However, the hypothesis of BCL2 rearrangement was supported by Dako BCL2 split probe finding of abnormal FISH pattern 1R2F.

Conclusions: Presented results show that due to slight construction differences Vysis and Dako rearrangement FISH probes may not provide equal results in all types of BCL2 rearrangement. Supported by Grant of Comenius University No. UK/432/2008.

\section{$\underline{\text { LS } 127}$}

\section{STRONG IMMUNOHISTOCHEMICAL EXPRESSION OF HEAT-SHOCK PROTEINS (HSPS) IN CLASSIC HODGKIN'S LYMPHOMA (cHL)}

* A Santón, A Pascual, E Cristóbal, J García-Laraña, M García-Cosío.

Hospital Ramón y Cajal, Madrid, Spain

Background: HSPs comprise several families of proteins which besides functionig as molecular chaperones play a pivotal role in apoptosis inhibition and cell survival. Over-expression of HSPs have been reported in many tumor types and associated with chemo-resistance and worse prognosis. However, the immunohistochemical (IHC) profile of HSP expression in $\mathrm{cHL}$, together with its possible role in the apoptosisresistant phenotype of tumor $\mathrm{H} / \mathrm{RS}$ cells are not well established.

Purpose: To analyze the IHC expression of main HSP families and the heat-shock transcription factor-1 (HSF-1) in cHL and their possible relationship with caspase signaling pathways.

Methods: Tissue-microarrays containing duplicate tumor cores from 100 pre-treatment-cHL cases were stained with antibodies against HSF1; HSPs 27, 32, 60, 70, 90 and 110, and co-chaperones HSP10, HSP40 and cdc37. To determine the status of intrinsic and extrinsic apoptotic pathways in H/RS cells, IHC expression of active caspases 3, 8 and 9, cleaved PARP-1/p89, c-FLIP, XIAP, and Bcl-2 was also evaluated.

Results: Almost all cHL cases showed strong expression of HSF-1 (100\%), HSP10 (100\%), HSP32 (95.5\%), HSP60 (98\%), HSP105 (99\%), and cdc37 (93\%). The percentage of cases showing positive staining for the remaining HSPs was lower: 39\% for HSP27, 66\% HSP $40,50 \%$ HSP70, and 58\% HSP90. Active caspases 3, 8, and 9 were detected in $100 \%, 56 \%$, and $97 \%$ of cases, respectively. Despite the detection of active caspase- 3 in all samples, cleaved PARP-1 (the main product of caspase-3 proteolytic activity) was observed only in $15.5 \%$ of cases. Apoptosis inhibitors Bcl-2, XIAP, and c-FLIP were expressed in $61 \%, 74 \%$, and $66 \%$ of biopsies, respectively.

Conclusions: Most cHL cases show strong IHC expression of HSF-1 and HSPs in tumor cells. Although functional studies are indeed required, expression of HSPs could contribute to unproper functioning of apoptotic machinery in HRS cells, as it happens in other tumor types.
LS 128

\section{ASSOCIATION BETWEEN HEAT SHOCK PROTEIN EXPRESSION AND THE PRESENCE OF EPSTEIN-BARR VIRUS IN CLASSIC HODGKIN'S LYMPHOMA}

* M García-Cosío, E Cristóbal, A Pascual, J García-Laraña, A Santón.

Hospital Ramón y Cajal, Madrid, Spain

Background: Heat shock proteins (HSPs) are proteins that play important roles in regulating cellular homeostasis and responses to stress stimuli including viral infections. Viruses use HSPs to facilitate takeover of the cellular DNA replication machinery and HSPs associate with key viral products that control cell cycle progression. The Epstein-Barr virus (EBV) is commonly associated with classic Hodgkin's lymphoma (cHL), especially the mixed-cellularity subtype, where is detected in the neoplastic H/RS cells. It remains in a latent state limiting expression to few genes. Among them, the most abundantly expressed are the 2 EBV-encoded small RNAs (EBERs) of which 1-10 million copies are transcribed per H/RS cell.

Purpose: To analyze the possible relationship between EBV and HSP expression in H/RS cells of cHL.

Methods: Tissue-microarrays containing duplicate tumor cores from 100 pre-treatment-cHL cases were stained with antibodies against HSF-1; HSPs 27, 32, 60, 70, 90 and 110, and co-chaperones HSP10, HSP40 and cdc37. Presence of EBV was assessed by EBER in situ hybridization. As EBERs have been shown to up-regulate Bcl-2 and to increase resistance to apoptosis, immunohistochemical (IHC) expression of c-FLIP, XIAP, p21, p53, and Bcl-2 was also evaluated. Results: EBER detection was possible in 88 o 100 cases. EBV was found in the neoplastic H/RS cells in 37 of them (42\%). Statistical analysis showed significant association between the presence of EBV and the expression of HSP $27(\mathrm{p}=0.009)$ and HSP $70(\mathrm{p}=0.030)$ in H/RS cells.

Conclusions: HSP 27 and HSP70 expression seems to correlate with latent EBV infection in malignant cells of $\mathrm{cHL}$, as it has been reported for other viral infections like HIV.

\section{MS 129}

\section{GENE PROFILING COMBINED WITH TMA ANALYSIS OF CLASSICAL HODGKIN LYMPHOMA UNCOVERS A SPECIFIC TH1/ANTIVIRAL RESPONSE IN EBV+ TISSUES AND IDENTIFIES THE B-CELL/ PLASMACYTOID DENDRITIC CELL-SPECIFIC GENE BCL11A AS A POWERFUL IHC MARKER OF FAVOURABLE OUTCOME.}

B Chetaille, F. Bertucci, P Finetti, B Esterni, A Stamatoullas, JM Picquenot, MC. Copin, F Morschhauser, O Casasnovas, T Petrella, T Molina, A Vekhoff, P Feugier, R Bouabdallah, L. Xerri*.

Institut Paoli-Calmettes, Marseille, France; and GELA, Rouen, Lille, Dijon, Paris, Nancy, France

Background: After treatment for classical Hodgkin lymphoma (cHL), about $40 \%$ of patients present either refractory disease or relapse. Identification of these patients who would require more intensive therapy is thus a crucial challenge. 
Material and Methods: A training set of $63 \mathrm{cHL}$ frozen tissue samples was profiled using Affymetrix DNA microarrays. We searched for gene expression signatures associated with relevant features of disease, including EBV status and survival. Ten tissues samples T-cell rich B-cell lymphoma (TCRBCL) and from benign adenitis were included as controls. A validation set of paraffin tissue samples from $146 \mathrm{cHL}$ patients (median follow-up of 77.8 months) was analyzed using immunohistochemistry (IHC) on tissue microrrays (TMAs).

Results: Unsupervised clustering showed that cHL samples were more heterogeneous than were TCRBCL and adenitis samples. Supervised analysis on the basis of the EBV status tissues provided a highly significant gene signature of 425 genes, showing that EBV+ HL tissues overexpressed genes characteristic of Th1 and antiviral responses including IFNG. A signature of 38 genes were significantly overexpressed in cHL patients with complete remission, when compared to those with refractory disease or relapse. These genes were involved in the function of B-cells and plasmacytoid dendritic cells (pDCs), like BCL11A. IHC validation on TMAs confirmed that high numbers of BCL11A + reactive cells, using both univariate and multivariate Cox models, correlated with both overall survival (OS) and event free survival (EFS) $(\mathrm{p}<0.001)$. Multivariate Cox model showed a significant correlation between OS and the IHC expression of FOXP-3, CD20 and topoisomerase IIA. No prognostic value was found for IHC detection of BCL2, LMP-1, PD-1, CD57, tryptase, granzyme-B, PS100, CD8, STAT1 and CD123.

Conclusions: Identification of BCL11A as a powerful IHC prognostic marker might help to select cHL patients who would benefit from reduced therapy. Our results indicating a Th1 and anti-viral response in $\mathrm{EBV}+\mathrm{HL}$ tissues provide a basis for novel treatment strategies.

\section{LS 130}

\section{EXPRESSION PROFILING OF 554 MRNA SPECIES CODING FOR ENZYMES INVOLVED IN GLYCOSYLATION OF PROTEINS IDENTIFY A PLASMA CELL DIFFERENTIATION SIGNATURE IN INDOLENT B-CELL LYMPHOMAS}

Barbara Petit ${ }^{1,2}$, Nathalie Gachard ${ }^{2,3}$, Lionel Forestier ${ }^{4}$, Sophie Duchez $z^{2},{ }^{5}$ Dominique Bordessoule, Raymond Julien ${ }^{4}$, Jean Feuillard ${ }^{2,3}$.

${ }^{1}$ Laboratoire d'Anatomie Pathologique and ${ }^{2}$ UMR CNRS 6101, ${ }^{3}$ Laboratoire d'Hématologie, ${ }^{4}$ UMR INRA 1061, ${ }^{5}$ Service d'Hématologie Clinique, Dupuytren Hospital and University of Limoges, France

Background: Glycosylation is essential for maturation of numerous proteins, particularly immunoglobulins, a key feature of plasma cell differentiation, which significance is matter of debate in indolent Bcell lymphomas, mainly in marginal zone lymphomas (MZL).

Material and methods. To asses the expression profile of 554 glycosylation related genes (glycogenome) in indolent B-cell lymphomas, we have designed 3 different TaqMan Low Density Arrays for high throughput QPCR. Total RNA was extracted from a series of 35 cases of B-CLL, Follicular Lymphomas (FL), MZLs of lymph node and spleen, MALT, lymphoplasmacytic lymphomas (LPL), and plasmocytoma (PL) and of 10 cases of reactive lymphoid tissues and/ or purified B-cell suspensions from tonsil, lymph nodes and spleens. Results: Supervised analysis led to define a set of 168 significant genes among the 484 (87\%) expressed in at least $75 \%$ of patients. Clustering of patients and controls with these 168 genes showed that both B-CLL and
PL were correctly segregated. FLs were clusterised with purified B-cells from lymph nodes. MZL were separated in 2 groups, roughly equal in size, those close to plasmocytoma and those close to B-CLL. A glycogenome plasma cell signature was clearly identified with both down-regulated genes such as CD22, CD69, CD72, CD83 or the glucose transporters GLUT1 and GLUT3 and up-regulated genes such as CD138/SDC1, or the mannosyltransferase ALG1, ALG2 and ALG3, essential for protein $\mathrm{N}$-glycosyslation in the endoplasmic reticulum (ER) or the mannosidase MAN1A1. Moreover, 3 genes, CHST11, LGALS1 and NDST1 were found to be expressed specifically in MZL and/or LPL. Conclusion: Analysis of the glycogenome revealed that up-regulation of genes coding for enzymes essential for mannosylation of proteins in the ER constitute a signature of plasma cell differentiation and that such signature separate MZLs in 2groups, those with and those without plasma cell differentiation features, independently of the organ origin of the tumour.

\section{LS 131}

\section{EXPRESSION OF PLASMA CELL DIFFERENTIATION MARKERS CD38, CD138 AND MUM1/IRF4 AND OF ZAP70 SUGGESTS THAT MARGINAL ZONE AND LYMPHOPLASMACYTIC LYMPHOMAS FROM LYMPH NODES MAY CORRESPOND TO A MORPHOLOGICAL SPECTRUM OF THE SAME ENTITY.}

${ }^{1}$ Isabelle Soubeyran ${ }^{1}$, Marie Parrens ${ }^{2}$, Barbara Petit ${ }^{3}$, Nathalie Gachard $^{4}$, Valérie Coste-Martineau ${ }^{5}$, Camille Laurent ${ }^{6}, \mathrm{~F}_{\text {Labrousse }}{ }^{3}$, D Bordessoule $^{7}$, Jean-Philippe Merlio ${ }^{2}$, Antoine de Mascarel ${ }^{2}$, J Feuillard ${ }^{4}$.

${ }^{1}$ Department of Pathology and INSERM U916, Institute Bergonié, Bordeaux;

${ }^{2}$ Department of Pathology and Molecular Biology, EA 2406 CHU of Bordeaux ;

${ }^{3}$ Department of Pathology and UMR CNRS 6101, CHU Dupuytren, Limoges;

${ }^{4}$ Laboratory of Haematology, and UMR CNRS 6101, CHU Dupuytren, Limoges;

${ }^{5}$ Department of Pathology, Hôpital Gui de Chauliac, Montpellier;

${ }^{6}$ Department of Pathology, Hospital Purpan, and INSERM-U563, Toulouse ;

${ }^{7}$ Department of Haematology and UMR CNRS 6101, Hôpital Dupuytren, Limoges;

Introduction: Among indolent lymphomas, Lymphoplasmacytic Lymphomas (LPL) and Marginal Zone Lymphomas (MZL) are still very difficult to separate when originated from lymph nodes (LN) due to both cellular heterogeneity and frequent features of plasma cell differentiation (PCD) in MZLs. We raise the question of the interest of immunophenotypic PCD markers to separate these 2 categories of tumours. Moreover, we studied expression of the tyrosine kinase ZAP70, a surrogate marker of IgVH mutation status in CLL.

Material and Methods: We have designed a Tissue-Micro-Array of 250 cases of MZLs and LPLs, among them a series of 38 CD20pos CD5neg CD23neg cases from LNs. Nine were diagnosed as LPL, based on a diffuse infiltration pattern with PCD, and 29 as nodal MZL (NMZL), based mainly on the persistence of nodularity, residual germinal centers and/or presence of monocytoid/marginal zone B-cell morphology. 
Results: Among NMZL, 58\% showed no significant, 21\% intermediate and 21\% marked morphological PCD. Among LPL, 22\% showed intermediate and $78 \%$ marked PCD. Expression of the 3 markers CD38, CD138 and MUM1/IRF4 was significantly associated, CD138 and MUM1/IRF4 being the most closely associated together $\left(p=2.10^{-4}\right)$. Expression of these 3 markers was significantly associated with PCD whatever the morphological diagnosis. Interestingly, marked expression of CD138 and/or MUM1/IRF4 was found in $31 \%$ of NMZLs with no morphological PCD. Expression of ZAP70 was negative in all cases; even in $4 \mathrm{IgVH}$ unmutated cases among 12 tested. Conclusion: CD38, CD138 and MUM1/IRF4 expression did not allow to separate LPL and MZL from LN. Moreover, these markers revealed a subset of NMZL with marked immunophenotypic features of PCD despite the lack of significant morphological PCD. Finally, ZAP70 was constantly negative in these cases, whatever the PCD and the IgVH mutational status. Altogether, these results suggest that LPLs and MZLs from lymph nodes correspond to a morphological spectrum of the same entity.

\section{$\underline{\text { LS } 132}$}

\section{LMO2 GERMLINE GENETIC VARIATION IS ASSOCIATED WITH LMO2 TUMOR EXPRESSION IN DLBCL}

*A. Dogan ${ }^{1}$, Y. Natkunam ${ }^{2}$, L. Morton ${ }^{3}$, M. Maurer ${ }^{1}$, T. Habermann ${ }^{1}$, S. Chanock $^{3}$, W. Cozen ${ }^{4}$, C. Lynch ${ }^{5}$, R. Severson ${ }^{6}$, C. Allmer ${ }^{1}$, I. Lossos ${ }^{7}$, R. Levy ${ }^{2}$, N. Rothman ${ }^{3}$, S. Slager ${ }^{1}$, P. Hartge ${ }^{3}$, S. Wang ${ }^{3}$, J. Cerhan ${ }^{1}$.

${ }^{1}$ Mayo Clinic, Rochester, MN, USA; ${ }^{2}$ Stanford U, Stanford, CA; ${ }^{3} \mathrm{NCI}$, Bethesda, MD; ${ }^{4} \mathrm{USC}$, Los Angeles, CA; ${ }^{5} \mathrm{U}$ of Iowa, Iowa City, IA; ${ }^{6}$ Wayne State, Detroit, MI; ${ }^{7} \mathrm{U}$ of Miami, Miami, FL.

Background: Both $L M O 2$ mRNA and LMO2 protein expression in DLBCL have been associated with better survival. In a populationbased sample of DLBCL cases, we evaluated the association between LMO2 germline variation and LMO2 protein expression.

Methods: We studied 186 DLBCL patients who participated in a population-based study conducted from 1998-2000 using cancer registries in Detroit, Seattle, Iowa, and Los Angeles. Immunohistochemistry for LMO2 was conducted on 5 micron section of tumor tissue from diagnostic biopsies, and results were read by two independent pathologists. Germline DNA was extracted from a venous blood sample or a buccal cell sample, and 20 single nucleotide polymorphisms (SNPs) from LMO2 were genotyped using the Illumina GoldenGate platform

Results: LMO2 protein was expressed in $46 \%$ of patients; expression was associated with better survival $(\mathrm{HR}=0.61 ; 95 \%$ CI $0.35-1.07, \mathrm{p}=$ 0.08). Two SNPs (rs941940 and rs7941248) in the promoter region of LMO2 were associated with protein expression in the tumor. Patients with $\mathrm{rs} 941940_{\mathrm{AA}}(66 \%)$ or $\mathrm{rs} 7941248_{\mathrm{CT} / \mathrm{TT}}(55 \%)$ genotypes were more likely to have tumors that expressed LMO2 than $\mathrm{rs} 941940_{\mathrm{AG} / \mathrm{GG}}$ (41\%), and rs $7941248_{\mathrm{CC}}(36 \%)$ genotypes, $\mathrm{p}=0.009$ and $\mathrm{p}=0.01$ respectively.

Conclusions: Genetic variation in $L M O 2$ promoter SNPs correlates with LMO2 protein expression. This data suggests that some of the fundamental biological characteristics of DLBCL, such as germinal center phenotype, may be determined by host genetic background.
LS 133

\section{MONOCLONAL ANTIBODY IMMUNOMODULATOR- RELATED LYMPHOID PROLIFERATIONS (MAIR-LPD)}

*Dennis P. O’Malley, M.D. Clarient Inc., Aliso Viejo, CA. Steve Chen, M.D. Clarient Inc., Aliso Viejo, CA. Sherrie L. Perkins, M.D.,

Ph.D. University of Utah, Salt Lake City, UT. Robert P. Hasserjian, M.D. Massachusetts General Hospital, Boston, MA, Marsha Kinney, M.D. University of Texas Health Science Center, San Antonio Texas, Todd Barry, M.D. PhenoPath, Seattle, WA, Jonathan Said, M.D. University of California, Los Angeles, CA, Megan Lim, M.D., Ph.D. University of Michigan, Ann Arbor, MI, William J. Finn, M.D. University of Michigan, Ann Arbor, MI, Matthew Snyder, M.D. WakeMed, Raleigh, NC.

Introduction: Monoclonal antibodies therapies have been successful for autoimmune disorders. These targeted therapies are designed to modulate the immune response. As with all therapies, these new monoclonal antibody (Mab)-based therapies are not without side effects and complications. One specific class of these therapies, anti-tumor necrosis factor (TNF) agents, has been reported to be associated with lymphoproliferative disorders. Further, many of the other Mab agents are also associated with lymphoproliferative disorders. We evaluate the clinicopathologic features of 10 cases of monoclonal antibody immunomodulator-related lymphoproliferative disorders (MAIR-LPD).

Materials and methods: Cases were obtained from several institutions. In all cases, clinical information and pathologic material was reviewed with respect to institutional safeguards for patient privacy. Demographic information, medication information (type, duration), and available follow-up information were reviewed. H\&E stained sections and immunohistochemical stains were reviewed. In cases where paraffin block were available, additional studies were performed. Studies for EBV (EBER) and PCR studies for IgH rearrangements, and when appropriate, TCR, were evaluated.

Results: The cases submitted illustrate a broad range of lymphoproliferative disorders. A variety of autoimmune disorders is represented and highlights the effects of Mab agents. A summary of results are presented in Tables 1 and 2.

Discussion: This case series illustrates a broad range of lymphoid proliferations associated with the Mab agents. The MAIR-LPD have considerable overlap with other iatrogenic lymphoid proliferations, such as those seen in post-transplant settings (e.g. PTLD) and those seen in association with methotrexate therapy. EBV was present in most B cell derived lymphoma types, while those "atypical" lesions or those of $\mathrm{T}$ cell derivation lack the EBV association. 
Table 1. Clinical information

\begin{tabular}{|c|c|c|c|c|c|c|c|c|c|}
\hline $\begin{array}{l}\text { Case } \\
\text { number }\end{array}$ & Age & gender & Presentation/clinical & Medication & Class & Disorder & Duration & $\mathrm{F} / \mathrm{U}$ & Outcome \\
\hline $1-$ & 40 & $\mathrm{M}$ & $\begin{array}{l}\text { Diffuse adenopathy, } \\
\text { night sweats, weight } \\
\text { loss }\end{array}$ & Daclizumab & TNF & MS & $2+$ years & Reduced dosage & $\begin{array}{l}\text { NED. } 9 \\
\text { mos. }\end{array}$ \\
\hline $2-$ & 49 & $\mathrm{~F}$ & Skin lesion & Infliximab & $\mathrm{TNF}$ & RA & NA & NA & \\
\hline 3 & 33 & M & & Infliximab & TNF & UC & NA & NA & \\
\hline 4 & 55 & M & Lymphadenopathy & Eflizumab & CD11a & Psoriasis & Unk & NA & \\
\hline 5 & 71 & $\mathrm{~F}$ & & Infliximab & TNF & $\begin{array}{l}\text { Crohn } \\
\text { Disease }\end{array}$ & 3 yrs. & Died & \\
\hline 6 & 63 & $\mathrm{~F}$ & & Infliximab & TNF & $\begin{array}{l}\text { Crohn } \\
\text { disease; } \\
\text { arthritis }\end{array}$ & NA & NA & \\
\hline 7 & 44 & M & Rectal mass & Infliximab & TNF & $\begin{array}{l}\text { Crohn } \\
\text { disease }\end{array}$ & 6 weeks & $\begin{array}{l}\text { R-CHOP x } \\
8 \text { cycles; NED } \\
7.5 \text { months }\end{array}$ & \\
\hline $\begin{array}{l}8 \\
\text { PAPA }\end{array}$ & 24 & M & $\begin{array}{l}\text { Enlarged lymph node } \\
\text { Syndrome }\end{array}$ & $\begin{array}{l}\text { Anti-TNF } \\
\text { unk }\end{array}$ & NA & & & (unspecified) & TNF \\
\hline 9 & 49 & M & $\begin{array}{l}\text { Skin lesion } \\
\text { Ankylosing spondylitis }\end{array}$ & $\begin{array}{l}\text { Anti-TNF } \\
\text { unk }\end{array}$ & NA & & & (unspecified) & TNF \\
\hline 10 & 55 & $\mathrm{~F}$ & Skin lesion & Etanercept & TNF & RA & unk & NA & \\
\hline
\end{tabular}

UC: ulcerative colitis, RA: rheumatoid arthritis, MS: multiple sclerosis

TNF: tumor necrosis factor, NA: not available, ND: not done

PAPA: Pyogenic arthritis, pyoderma gangrenosum, and acne

Table 2. Summary of pathologic findings

\begin{tabular}{|c|c|c|c|c|}
\hline $\begin{array}{l}\text { Case } \\
\text { number }\end{array}$ & Morphology & EBV & $\mathrm{IgH}$ & TCR \\
\hline 1 & Atypical hyperplasia & - (EBER) & - & - \\
\hline 2 & SPTCL & $\begin{array}{l}\text { - (EBER, } \\
\text { LMP) }\end{array}$ & $\mathrm{ND}$ & ND \\
\hline 3 & $\begin{array}{l}\text { Atypical T cell, } \\
\text { suspicious for PTCL }\end{array}$ & - (EBER) & - & - \\
\hline 4 & $\mathrm{FL}$, grade $2 / 3^{1}$ & + & $\mathrm{ND}$ & ND \\
\hline 5 & DLBCL & $+($ EBER $)$ & ND & ND \\
\hline 6 & CHL, LR & $+($ EBER $)$ & ND & ND \\
\hline 7 & DLBCL & $+(\mathrm{EBER})$ & $\mathrm{ND}$ & ND \\
\hline 8 & Atypical with necrosis & ND & NA & NA \\
\hline 9 & $\begin{array}{l}\text { Atypical: Nasal type } \\
\text { NK/TCL vs. } \\
\text { LyG }\end{array}$ & - (EBER) & Oligo & NA \\
\hline 10 & ALCL, ALK+ & - (EBER) & $\mathrm{ND}$ & ND \\
\hline
\end{tabular}

SPTCL: subcutaneous panniculitic T cell lymphoma, PTCL: peripheral T cell lymphoma, DLBCL: diffuse large B cell lymphoma, CHL: classical Hodgkin lymphoma, LR: lymphocyte-rich, EBER: EpsteinBarr virus early RNA, LMP: latent membrane protein LyG: Lymphomatoid granulomatosis, 1. Negative for $\mathrm{t}(14 ; 18)$, IgH, or bcl-6 translocations by FISH.

\section{LS 134}

\section{REGULATORY T-CELLS AND NK CELLS IN B-CELL POST-TRANSPLANT LYMPHOPROLIFERATIVE DISORDERS (B-PTLD), HIV-ASSOCIATED B-CELL LYMPHOMAS AND IMMUNE COMPETENT DIFFUSE LARGE B-CELL LYMPHOMAS.}

\author{
* Hazem AH Ibrahim, Lia Menasce, Mark Bower, and Kikkeri N \\ Naresh.
}

Departments of Histopathology, Hammersmith Hospital and Imperial College London, UK \& Christie Hospital, Manchester, UK.

Background: PTLDs are a group of lymphoproliferative disorders that develop in the background of immune suppression. Regulatory Tcells (T-regs) play an important role in the inhibition of transplant rejection and autoimmune disease. Fox-P3, a forkhead box transcription factor, has been shown to be expressed in T-regs. It has been shown that the T-regs can suppress the growth of some tumours in addition to its role in suppression of the anti-tumour immune response through inhibition of cytokine production by the tumour cells. The latter effect allows the tumour cells to escape the host anti-tumoural Tcell immune response. Natural killer (NK) cells are an important component of the innate immune response against tumours.

Purpose of the study: We intended to investigate whether B-PTLDs and HIV-associated B-cell lymphomas significantly differ from immunocompetent (IC) diffuse large B-cell lymphomas (DLBCL) with respect to infiltration by T-regs (Fox $-\mathrm{P}^{+}{ }^{+}$) and NK cells $\left(\mathrm{CD} 56^{+}\right)$. Description of the results: We carried out immunohistochemistry for expression of Fox-P3 and CD56 among 19 cases of B-PTLD, 14 HIVassociated B-cell lymphomas (7 Burkitt Lymphoma, 7 Diffuse large B-cell lymphoma (DLBCL)), and 37 cases of immunocompetent (IC)DLBCLs. The number of cells positive for Fox-P3 and CD56 was counted and the numbers were expressed as cells $/ \mathrm{mm}^{2}$. The number of 
Fox-P $3^{+}$cells $/ \mathrm{mm}^{2}$ among B-PTLD, HIV-BL, HIV-DLBCL and ICDLBCL was $159+94,53+54,142+90$ and $64+22$ respectively. BPTLD had higher Fox-P3 ${ }^{+}$cells as compared to IC-DLBCL $(p=0.08)$. The number of CD56 cells $/ \mathrm{mm}^{2}$ among B-PTLD, HIV-BL, HIVDLBCL and IC-DLBCL was $11+8,1+1,4+4$ and $1.5+1$ respectively. B-PTLD had significantly higher $\mathrm{CD}^{+} 6^{+}$cells positive cells as compared to IC-DLBCL $(\mathrm{p}=0.008)$.

Conclusion: Tissue samples from B-PTLD have significantly higher numbers of Fox-P3 and CD56 positive cells as compared to immunocompetent DLBCLs.

\section{$\underline{\text { LS } 135}$}

\section{THE MORPHOLOGIC OVERLAP BETWEEN HODGKIN LYMPHOMA AND OTHER LYMPHOMPROLIFERATIVE DISEASES. A WELL KNOWN BUT STILL DIFFICULT PROBLEM.}

Martin $\mathrm{CA}^{*}$, Sapia $\mathrm{S}^{+}$, Zanetto $\mathrm{U}^{\&}$, de Dios Soler $\mathrm{M}^{\#}$, Narbaitz $\mathrm{MI}^{\#}$.

From the Departments of Pathology, ${ }^{*}$ San Roque Hospital, ${ }^{+}$ Fundaleu, \# National Academy of Medicine, ARGENTINA; and \& City Hospital, Birmingham, UK.

The borderline between Hodgkin lymphoma (HL) and so called "NonHodgkin's Lymphomas" (NHL) is sometimes difficult to delineate. Recently, "Grey Zone Lymphomas" (GZL), from mediastinal and non-mediastinal origin have been described. We present our experience with a group of these lesions.

Cases of GZL from four institutions were retrieved. After careful evaluation, seven cases ( 5 males and 2 females, aged 12 to 63 years old) were selected and grouped according to the scheme of Stein (2005) as follows: one case was of type I, one of type II and 4 cases of type III. In addition, there was a case of a reactive lymphadenitis mimicking HL. GZL type I included a case of DLBLC coexisting with isolated foci of NLPHL at presentation. Type II included one case of HL with T-cell marker expression (CD30+, CD15+, CD3+, $\mathrm{CD} 5+, \mathrm{CD} 45+$ ) and reactivity for PAX5, with no TCR or $\operatorname{IgH}$ rearrangement. Type III group included a case of PMLBCL (CD30+, $\mathrm{CD} 15+)$ with strong positivity for CD20. Two further cases showed Reed-Sternberg and anaplastic large cells positive for CD20, CD15, CD30 and CD45. One of these was EMA+ and the other PAX5, MUM1 and Oct2 +ve. The remaining case showed a CD20+, TIA-1+ DLBCL with clusters of CD15+ R-S and Hodgkin cells. Lastly, the case of diffuse lymphadenitis featured large mononuclear CD30+ cells and a T-cell rich background. This was followed overtime and a diagnosis of HL was made in a second lymph node biopsy at another institution.

Lymphoid proliferations with borderline features between HL and NHL or reactive lesions are infrequent. The use of selected antibodies combined with meticulous morphological assessment allows for the correct diagnosis in most cases, and ancillary molecular studies permit correct assignment of lineage in more difficult ones. However, some cases remain difficult to classify. Newer strategies are needed to solve these unusual situations.
LS 136

TITLE

\section{THE ROLE OF HGAL PROTEIN EXPRESSION IN THE GERMINAL CENTER MICROENVIRONMENT}

C Temmins, $\mathrm{MD}^{1} \mathrm{~S}$ Zhao, MS ${ }^{1}$ IS Lossos, $\mathrm{MD}^{2} \mathrm{Y}$ Natkunam, MD, PHD $^{1}$

${ }^{1}$ Pathology, Stanford University School of Medicine, Stanford, CA; ${ }^{2}$ Sylvester Comprehensive Cancer Center, University of Miami, Miami, FL

Background: HGAL is a germinal center (GC) B-cell-specific marker whose expression is associated with a favorable outcome in diffuse large B-cell (DLBCL) and classical Hodgkin lymphoma (CHL). Mouse studies showed that HGAL expression is dispensable for GC formation and function; however, IL-6-induced phosphorylation of HGAL modulates lymphocyte migration, and may play a role in confining B-cells to the GC microenvironment.

Purpose and design: In order to characterize profiles of HGAL protein expression in disorders involving GC disruption, 75 cases including 23 progressive transformation of GC (PTGC), 25 follicle lysis (FLy) and 27 Castleman disease (CD: 17 hyaline-vascular (HV), 4 plasma cell (PC) and 6 multicentric (MC) variants), were studied. Results: HGAL protein expression uniformly correlated with the presence of GC B-cells in all categories including PTGC, Fly, PC-CD and MC-CD but was absent in a subset of HV-CD that showed severe regression or absence of GCs. HGAL staining highlighted dismantled GCs in PTGC; in majority of cases, pockets of GC B-cells were highlighted by HGAL in contrast to weak or absent CD10 and BCL6 staining. In FLy, HGAL expression was comparable to that of CD10, BCL6 and CD21 in highlighting lysed follicles; however, HGAL staining was stronger in florid FLy.

Conclusions: HGAL protein expression is intimately associated with GC B-cells and effectively discriminates clusters of GC B-cells in disrupted follicles. Its persistence in disrupted GCs in the absence of CD10 and BCL6 suggests that it may be necessary for GC maintenance. This expression pattern supports its proposed role in confining B-cells to the GC by modulating lymphocyte migration. Given that IL-6 plays a role in $\mathrm{CD}$, increased HGAL expression in extrafollicular plasmacytoid cells suggests a role for HGAL signaling in the pathogenesis of CD. The favorable outcome of HGAL-positive DLBCL and CHL patients is likely related to decreased dissemination (low stage disease) and suggests a putative role for HGAL in the GC microenvironment.

\section{LS 137}

JNK IS CONSTITUTIVELY ACTIVE IN MANTLE CELL LYMPHOMA: INHIBITION OF CELL PROLIFERATION AND CELL CYCLE PROGRESSION BY JNK INHIBITOR SP600125

*Miao Wang, Stefano Rosati, Cigdem Atayar, Edwin Spithoven, Hans Vos, Philip Kluin, Lydia Visser

Department of Pathology, University Medical Center Groningen, University of Groningen, Groningen, The Netherlands 
Mantle cell lymphoma (MCL), is a lymphoma with a poor prognosis for which any effective treatment is still lacking. Different signaling pathways are activated in MCL cells and may be possible targets for therapeutic intervention.

We investigated the role of the MAPK signaling pathway in MCL. Normal tonsillar B cells and MCL cells were isolated from cell suspensions by negative isolation and were used in a MAPK array. Paraffin embedded tissue of MCL cases was used for immunohistochemistry. MCL cell lines Jeko-1, HBL-2, UPN-1 and Granta-519 were used for functional studies.

We used a MAPK array to compare MAPK kinase activity in mantle cell lymphoma cells with normal B cells and found upregulation of JNK in MCLValidation by immunohistochemistry showed that phospho-JNK is overexpressed in $81 \%(30 / 37)$ of MCL cases. We concluded that JNK is constitutively activated in MCL lymphoma cases. Western blot analysis on MCL cell lines showed further upregulation of phospho-JNK upon stimulation with CD40L \& IL4 but not with anti-IgM. Inhibition of JNK activation with SP600125 resulted in growth arrest and apoptosis in all MCL cell lines, an effect that could be overcome by the addition of CD40L \& IL-4. This effect was JNK specific since inhibition of ERK or p38 did have any effect. Furthermore, we found that in MCL cell lines SP600125 leads to G2-M phase arrest on day 1 and to an obvious increase in endoreplication on day 2 and day 3 . All effects were p53 independent. G2-M arrest was probably induced by a concomitant downregulation of the transcription factor EGR-1. Endoreplication could be blocked by inhibition of Bcl-2, using the Bcl-2 inhibitor YC 137. These data suggest that basal JNK activity is required for the growth of MCL and plays an important role in progression of MCL cells through the cell cycle.

\section{LS 138}

\section{IMMUNOHISTOCHEMICAL PROFILING OF DIFFUSE LARGE B-CELL LYMPHOMAS IN A PROSPECTIVE RANDOMIZED TRIAL}

Heinz-Wolfram Bernd ${ }^{1 *}$, Michael Pfreundschuh ${ }^{2}$, , Marita Ziepert ${ }^{3}$, Alfred C. Feller ${ }^{1}$

${ }^{1}$ Institut für Pathologie, UKSH, Campus Lübeck, Germany; acting for the German Reference Pathology Centers for Lymph Node Pathology ${ }^{2}$ Klinik für Innere Medizin I, Universitätsklinik Homburg/Saar, Germany

${ }^{3}$ Institut für Medizinische Informatik, Statistik und Epidemiologie, IMISE, Universität Leipzig, Germany

The effords to develop a robust immunohistochemical classifier for prognostic class prediction in diffuse large B-cell lymphoma yielded in inconclusive and conflicting results so far. In this study, we performed IHC profiling in DLBCL cases from a large prospective randomized trial to possibly disclose prognostic subgroups and to test the published immunohistochemical classifiers in an independent prospective cohort.

In our study, 414 cases of DLBCL, all of which are enrolled in the NHL-B trial of the DSHNHL, have been immunohistochemically analyzed with a broad panel of antibodies using tissue micro arrays. Results were correlated to clinical data and compared to published classifiers.
Amongst other results, the presence of CD23-positive FDC meshworks, a clonal light chain secretion by the tumour cells and expression of HLA-DR emerged as independent markers of improved OS in multivariate analyses. By applying to all cases previous published algorithms for classification into a GCB and a non-GCB group, we observed a strong trend for better OS in the GCB cases, but this was not significant $(\mathrm{p}=0,055)$.

In conclusion, the previously reported significant IPI-independent prognostic value of class prediction according to suggested IHC classifiers could not be reproduced in our series. Results of several IHC studies are heterogeneous and disclose a limited reproducibility. Robust and reproducible immunohistochemical class prediction needs highly standarized work up and evaluation criteria that are universally accepted. None of the proposed IHC predictors is yet ready for clinical application.

\section{LS 139}

\section{IMMUNOHISTOCHEMICAL EXPRESSION OF CHECKPOINT KINASE-1 IN NORMAL HUMAN TONSILS AND BURKITT LYMPHOMA}

\author{
*S. Rosati, S. van Nierop, E.J. Boerma, P.M. Kluin
}

University Medical Center Groningen - Department of Pathology Groningen, The Netherlands

Background: In the germinal center (GC) dark zone, centroblasts proliferate despite AID-induced DNA damage. In the DNA damage response (DDR), checkpoint-kinase-1 (Chk1) is phosphorylated by ATR and triggers the S- and G2 phase checkpoints, phosphorylating TP53 and the CDC25 phosphatases. In centroblasts, Bcl- 6 attenuates the DDR by transcriptional repression of among others ATR. Bcl-6 inhibition restores ATR transcription, Chk1 phosphorylation and the DDR. Disabling the ATR-Chk1 signalling could favour the growth of rapidly proliferating lymphomas. This is supported by our unpublished array $\mathrm{CGH}$ data on Burkitt lymphoma (BL), showing a hemizygous deletion at 11q24 (Chkl locus) in 4/16 cases.

Purpose: Immunohistochemical investigation of Chk1 could contribute to understand its regulation and function. We determined Chk1 expression in 4 tonsils and $24 \mathrm{BLs}$, using IHC with an anti-Chk1 rabbit MoAb.

Results: in tonsils, Chk1 was almost exclusively expressed in the GC dark zone, with strong nuclear and weak cytoplasmic positivity. 19 BLs (including 2 with 11q-) could be evaluated. The $211 \mathrm{q}$ - cases showed minimal nucleocytoplasmic staining. 5/17 cases showed mainly or exclusively cytoplasmic expression. 12 cases showed nuclear and cytoplasmic positivity of variable intensity, including 5 cases with a strong expression similar to normal centroblasts.

Conclusion: by IHC on normal tonsils, Chk1 is seen mainly in proliferating cells and particularly in the dark zone centroblasts. Recent data suggest that Chk1 constitutively associates with chromatin; ATR-mediated Ser317 and Ser345 phosphorylations mediate, respectively, Chk1 release from chromatin and Chk1 activation, followed by nuclear export and proteasomal degradation. Our IHC findings are consistent with this model. Compared to centroblasts, in BLs the heterogeneous staining intensity and subcellular localization may reflect acquired abnormalities in gene dosage, phosphorylation or mutation. Currently we are performing IHC studies with antibodies against different phopshorylated forms of 
Chk1 (pChk1-Ser317, pChk1-Ser345 and pChk1-Ser280) to further explore Chk1 function.

\section{LS 140}

\section{NODULAR LYMPHOCYTE PREDOMINANT HODGKIN LYMPHOMA AND T CELL/ HISTIOCYTE RICH B CELL LYMPHOMA-CLINICAL, MORPHOLOGICAL AND IMMUNOPHENOTYPICAL FEATURE AND THEIR IMPORTANCE FOR DIFFERENTIAL DIAGNOSIS}

Dr. Aurelia Tatic, Dr. Iulia Ursuleac, Dr. Camelia Dobrea, Dr. Razvan Stoia, Dr. Emilia Niculescu Mizil, Dr. Bogdan Ionescu

Fundeni Clinical Institute, Department of Hematology

Background: grey zone lymphomas include those lymphoid malignancies that shows overlapping morphological, immunophenotypical features; main entities are : classical Hodgkin lymphoma, T cell/ histiocyte-rich large B cell lymphoma(TCHRLBCL), nodular lymphocyte predominant Hodgkin lymphoma (NLPHL). The diagnosis between these entities may be difficult, but is very important because the therapeutical options, the course and prognosis are different for each form of lymphoma. The incidence of TCHRLBCL and NLPHL is very low (6-7\% respectively $4-5 \%)$.

Method and material: in Fundeni Clinical Institute between 20022008, were diagnosed 4 cases of TCHRLBCL , 2 cases of NLPHL and 1 case of NLPHL transformed in TCHRLBCL from a total of 1653 malignant lymphomas ( 444 Hodgkin Lymphomas and 1209 non Hodgkin Lymphomas).

The NLPHL patients were males, with a median age of 39 years old, diagnosed in early stages(I B/Ann Arbor), based on clinical, morphological and immunophenotypical assessments; the course was favorable with ABVD chemotherapy and even at relapse(recorded in one case)the chemosensitivity was proven.

The TCHRLBCL patients were women, with a median age of 52,5 years old; all of them were diagnosed in advanced stages( IIIB, IVB/Ann Arbor) and were refractory to chemotherapy.

Conclusion: the differential diagnosis between TCHRLBCL and NLPHL is very important, because the evolution of disease and the responsiveness to chemotherapy is different; NLPHL has a more indolent course and good remission may be obtained with conventional chemotherapy. TCHRLBCL is an aggressive disease and should be treated with aggressive regimens of chemotherapy; despite of the treatment, complete response is rare and for short time installed.Because morphological appearance may be similar, immunohistochemistry is the main method for differential diagnosis. The incidence of these 2 entities among all of malignant lymphomas was very low ( $1 \%)$, but there is a question about the possibility of misdiagnosed cases ( confusion with other types of lymphomas).
LS 141

\section{TRANSLOCATIONS INVOLVING 8Q24 IN BURKITT LYMPHOMA AND OTHER MALIGNANT LYMPHOMAS - A HISTORICAL REVIEW OF CYTOGENETICS IN THE LIGHT OF TODAYS KNOWLEDGE}

\author{
EG Boerma $^{1}$, R Siebert ${ }^{2}$, PhM Kluin ${ }^{1}$, M Baudis $^{3}$
}

${ }^{1}$ Department of Pathology and Laboratory Medicine, University Medical Center Groningen, Groningen, The Netherlands, ${ }^{2}$ Institute of Human Genetics, University Hospital Schleswig-Holstein, Campus Kiel, Christian-Albrechts-University, Kiel, Germany, ${ }^{3}$ Institute of Molecular Biology, University of Zurich, Zurich, Switzerland

Burkitt lymphoma (BL) has a characteristic translocation $\mathrm{t}(8 ; 14)(\mathrm{q} 24$; q32) or its variants, however, diagnostic dilemmas occur as there is overlap with other lymphomas such as a subset of diffuse large B cell lymphomas (DLBCL). Recently two gene expression studies found that $\mathrm{BL}$ has a distinct molecular profile, but also that some cases intermediate between BL and DLBCL exist. An alternative approach is to consider chromosomal abnormalities. We explored the "Mitelman database" to define a cytogenetic profile of "true" Burkitt lymphoma. The core subset of $481 \mathrm{BL}$ showed a very low complexity of chromosomal changes, $40 \%$ of the cases having the $8 \mathrm{q} 24$ translocation with IG-MYC fusion as a sole abnormality. In the remaining cases recurrent, partially exclusive abnormalities included gains at chromosomes 1q, 7 and 12, and losses of 6q, 13q32-34 and $17 \mathrm{p}$. No differences existed between the 205 pediatric and 215 adult BL. This profile was different from that of the 13 BL with an 8q24 breakpoint not affecting the IG loci, the $44 \mathrm{BL}$ that had a second translocation involving a breakpoint at 18q21, 3q27 or 11q13 (doublehit lymphomas), and other morphological types of lymphomas with an $8 \mathrm{q} 24$ breakpoint $(\mathrm{N}=327)$. These lymphomas showed a much higher cytogenetic complexity than the core subset. BL without a cytogenetically detectable $8 \mathrm{q} 24 / \mathrm{MYC}$ breakpoint $(\mathrm{N}=108)$ also had a higher complexity than the core BL but might be heterogeneous and therefore deserves further studies. We conclude that a diagnosis of BL should be restricted to cases with a proven IG-MYC co-localization and a typical profile of imbalances without evidence of a double-hit and that classical cytogenetic analysis is of supplementary diagnostic value. Secondly, since the age distribution shows a steady increase of lymphomas that mimic BL, there is no distinct age at which a pathologist can safely make a diagnosis of BL without any ancillary studies.

\section{LS 142}

FOXP3+ REGULATORY T-CELLS IN ANGIOIMMUNOBLASTIC T-CELL LYMPHOMA (AITL): ARE THEY CORRELATED WITH THE BIOLOGY OF THIS LYMPHOMA ? IMMUNOPHENOTYPING STUDY BY FLOW CYTOMETRY AND IMMUNOHISTOCHEMISTRY.

J Bruneau (1); V Asnafi (1); L Lhermitte (1); E Macintyre (1); N Brousse (2); * D Canioni (2);

(1) Hematobiology Department, (2) Pathology Department, Hôpital Necker, Paris, FRANCE 
Background: AITL is a distinct clinicopathological entity among peripheral T-cell lymphomas. The abnormal infiltrate is constituted of atypical $\mathrm{T}$ lymphocytes $\mathrm{CD} 4+, \mathrm{CD} 10+, \mathrm{Bcl6}^{+}$admixed with an abundant reactive micro-environment cells. The cellular origin of AITL seems to derive from follicular helper T-cells but little is known about AITL biology. These patients have immune dysfunctions appearing to be secondary to the neoplastic process rather than preceding it. $\mathrm{CD} 4+, \mathrm{CD} 25+, \mathrm{FOXP} 3+$ regulatory T-cells (Tregs) represent important lymphoma/host environment modulators and their role in the regulation of immune response appears more and more predominant.

Purpose of the study: We assessed the role of Tregs number in AITL lymph nodes (LN) by an immunohistochemical study performed by flow cytometry (FCM) on 33 cases (5 AITL, 8 reactive LN, 20 B-cell NHL) and on formalin-fixed biopsies on 23 cases (15 AITL, 3 reactive LN, 3 follicular NHL and 2 large B-cells NHL) using FOXP3 antibody (Abcam,1/40). The Tregs were evaluated on slides by counting stained nuclei on five different fields on high power field $(\times 400)$.

Results: FCM revealed that the median infiltration of Tregs / CD4+ CD25- T-cells was statistically different between reactive LN: $2.9 \%$ $(2.3 \%-6.5 \%)$ and B-cells NHL: $11.4 \%(0.7 \%-25.4 \%)$ and between Bcells NHL and AITL: $2.4 \%(0.3 \%-7.5 \%)(p<0.01)$. Immunohistochemistry on slides confirmed the significant difference of nuclei FOXP3+ number stained in AITL: 82 (51-121) comparing to B cell NHL: 251 $(166-298)(p<0.01)$ and was less significant between reactive LN :196 $(140-265)$ and AITL $(\mathrm{p}<0.05)$. FCM revealed also that tumoral T cells CD4+, CD10+ were CD25- and FoxP3-

Conclusion: The alteration of T-cell regulators lymphoid functions seems to be implicated in AITL biology. This decrease of Tregs number may favor the development of AITL, or may be secondary to AITL. This study could also propose new targeted therapies for this lymphoma of bad prognosis.

\section{LS 143}

\section{MICRORNA SIGNATURES OF DIFFUSE LARGE B-CELL LYMPHOMAS AND FOLLICULAR LYMPHOMAS}

Christoph Thorns ${ }^{1}$, Anja Roehle ${ }^{1}$, Kai P Hoefig ${ }^{1}$, Dirk Repsilber ${ }^{2}$, Marita Ziepert ${ }^{6}$, Markus Loeffler ${ }^{6}$, Wolfram Klapper $^{3}$, Michael Pfreundschuh $^{4}$, András Matolcsy ${ }^{5}$, Heinz-Wolfram Bernd ${ }^{1}$, Lila Reiniger ${ }^{5}$, Hartmut Merz ${ }^{1} \&$ Alfred C Feller ${ }^{1}$

1 Institute for Pathology, University Clinic Schleswig-Holstein, Campus Luebeck, Germany

2 Research Institute for the Biology of Farm Animals FBN, Dummerstorf, Germany

3 Institute for Pathology, University Clinic Schleswig-Holstein, Campus Kiel, Germany

4 Department of Internal Medicine I, University Clinic Saarland, Homburg / Saar, Germany

5 1st Department of Pathology and Experimental Cancer Research, Faculty of Medicine, Semmelweis University, Budapest, Hungary 6 Institute for Medical Informatics, Statistics and Epidemiology IMISE, University Leipzig, Leipzig, Germany

MicroRNAS (miRNA, miR) are small ( 22nt) RNAs that function as translational repressors by binding to the $3^{\prime} \mathrm{UTR}$ of the target mRNA. They play key roles in many cellular pathways and can have tumour suppressor and oncogenic properties. Recent data from Lawrie et al. suggest that miRNAs are differentially expressed in the germinal center B cell-like and activated B cell-like subtype of diffuse large Bcell lymphomas (DLBCL) and may serve as prognostic markers (Lawrie et al.: Br J Haematol 2008; 141:672-5 and Int J Cancer 2007; 121:1156-61).

Here we analyzed the expression signatures of 157 miRNAs in 58 DLBCL, 46 follicular lymphoma (FL) and seven non-neoplastic lymph nodes (LN). Comparison of DLBCL-, FL- and LN resulted in specific DLBCL- and FL-signatures, which include miRNAs with previously published function in haematopoiesis (miR-150 and miR155 ) or tumour development (miR-210, miR-10a, miR-17-5p and miR-145). As compared to lymph nodes, some miRNAs are differentially regulated in both lymphoma types (miR-155, miR-210, miR-106a, miR-149 and miR-139), whereas other miRNAs seem to be differentially expressed in a lymphoma-specific manner such as miR-9/9*, miR-301, miR-338 and miR-213 in FL and miR-150, miR17-5p, miR-145, miR-328 and others in DLBCL. In a multivariate analysis adjusted for IPI-factors, a group of eight miRNAs were identified, that are of prognostic relevance for tumour outcome. Reduced expression levels of six miRNAs (miR-19a, miR-21, miR23a, miR-27a, miR-34a and miR-127) reveal poor event-free survival (EFS) and/or overall survival (OS), whereas the opposite is true for the remaining two (miR-195 and let-7g). Only miR-127 significantly influences both OS and EFS in a multivariate analysis.

In conclusion DLBCL and FL show different miRNA-signatures. Moreover, a rather low number of miRNAs seem to be of prognostic relevance for tumour outcome in DLBCL in a multivariate analysis.

\section{LS 144}

\section{HISTOPATHOLOGICAL AND IMMUNOHISTOCHEMICAL FEATURES OF HIGH-GRADE TRANSFORMATION OF 20 CASES OF LOW-GRADE B-CELL LYMPHOMAS}

*Mine Hekimgil ${ }^{1}$, Nazan Ozsan ${ }^{1}$, Neslihan Incili $^{1}$, Seckin Cagirgan ${ }^{3}$, Murat Tombuloglu ${ }^{3}$, Guray Saydam ${ }^{3}$.

${ }^{1}$ Department of Pathology and ${ }^{3}$ Hematology, Ege University Faculty of Medicine, Izmir, Turkey.

Several independent studies have already reported progression to diffuse large B-cell lymphoma (DLBCL) in indolent lymphomas such as follicular lymphoma (FL), marginal zone lymphoma of MALT type (MALT lymphoma), chronic lymphocytic leukemia/small lymphocytic lymphoma (CLL/SLL), and nodular lymphocyte predominant Hodgkin lymphoma (NLPHL). The aim of the study is to describe the histopathological and immunohistochemical features of high-grade transformation in patients previously diagnosed and treated as lowgrade lymphomas. From 1990 to 2008, 20 patients with low-grade lymphomas were diagnosed with biopsy-proven transformation, all to DLBCL, except one case with CLL/SLL which had transformed to Hodgkin lymphoma (HL). The initial diagnoses were FL in three, MALT lymphoma in four, NLPHL in five, and CLL/SLL in eight cases. The median follow-up period between the initial diagnosis of low-grade lymphomas and transformation to high-grade disease was 2 years. Of the FL cases one with grade 1 and the other with grade 3 disease have relapsed with diffuse large cell morphology within 2 and 3 years, respectively. Interestingly, the third case was diagnosed as DLBCL initially, and received the diagnoses of FL grade 1 within a 
month before the initiation of chemotherapy. All four cases of MALT lymphoma presented with a high-grade morphology on initial endoscopic biopsies. All of the five cases of NLPHL relapsed with DLBCL within 2 to 9 years of follow-up. Of eight cases of CLL/SLL, one presented prolymphocytic transformation and was followed-up for four years with multiple biopsies which were all diagnosed as infiltration of CLL/SLL, two presented Richter transformation to DLBCL on the year of diagnosis, and the rest relapsed within 1 to 4 years after diagnosis as DLBCL and interestingly one as HL. Our cases and review of the literature suggest that large-scale research is needed to validate the biological signals that influence the high-grade transformation of indolent lymphomas.

\section{$\underline{\text { LS } 145}$}

\section{THE THYMUS DURING HODGKIN LYMPHOMA AND PRIMARY MEDIASTINAL B LYMPHOMA DEVELOPMENT: A LIGHT MICROSCOPICAL AND IMMUNOHISTOCHEMICAL STUDY}

\author{
*M. Marino (1), S. Buglioni (1), A. Papadantonakis (1), R. Martucci (1), E. \\ Gallo (1), F. Pisani (2), F. Facciolo (3) and R. Perrone- Donnorso (1)
}

1) Dept. of Pathology, Regina Elena National Cancer Institute, Rome, Italy

2) Hematology, Regina Elena National Cancer Institute, Rome, Italy

3) Thoracic Surgery, Regina Elena National Cancer Institute, Rome, Italy

Background- Distinct lymphoma entities among NHL, such as Primary Mediastinal B Lymphomas (PMBL), and also Hodgkin Lymphomas (HL) arise in the thymus. However, due to destructive lymphoma growth, very limited informations are available concerning thymus changes during lymphoma development or spreading. However, the origin in the thymus of both HL and PMBL has been related to thymic lymphoid cells, and, for PMBL, to CD23+ large asteroid B cell in the thymic medulla.

Study purpose- We show here the pathological features of 2 cases of primary lymphomas ( $1 \mathrm{HL}$ and $1 \mathrm{PMBL}$ ) developing in the thymus, simulating during preoperative investigations a thymic epithelial cell (TEC) tumor and resected by radical thymectomy.

Results- Case 1 - A case of partially cystic HL occurred in the anterior mediastinum of a 63 year-old woman. In the thymic tissue distant from the tumor a distinctive type of lymphoid nodule was seen "budding" from the medullary border in the adipous tissue or, elsewhere, "in" the medulla, formed by small monotonous B cells and a central focus of large atypical CD30+ cells . The network of medullary TEC was surrounding this central nodule and it was dislocated from the nodular growth. Case 2 - The case of PMBL occurred in a 42-year old woman and it simulated a thymic carcinoma, both macroscopically and, at first glance, microscopically. The CD20+, CD23+ lymphoid cells, coexpressing BCL6 and MUM1/IRF4, grew in a cohesive way, surrounding areas containing small mature lymphocytes, simulating thymoma "perivascular areas". These "perivascular areas", by immunohistochemistry, were found to be medullary remnants . At tumor periphery, normal medullary thymic tissue was seen, compressed and focally invaded from the "lymphoid" tumor ; CD23+ lymphoid emboli were seen in the vessels of corticomedullary junction.

Conclusions Our morphological observations in 2 lymphoma cases could contribute informations about the origin of HL and PMBL from thymic lymphoid cells, the early spreading and the microenvironmental features associated to lymphoma development .

\section{LS 146}

\section{LYMPHOPROLIFERATIVE DISEASES IN MYELODISPLASTIC AND /OR SPLENOMEGALIC PATIENTS: A MULTIDISCIPLINARY DIAGNOSTIC APPROACH}

*M. Marino (1), L. Dessanti (2), M. D’Andrea (2), S. Buglioni (1), R. Martucci (1), P. Canalini (1), S. Conti (1), E. Vico (3), R. PerroneDonnorso (1) and I. Cordone (4)

1) Dept . of Pathology, Regina Elena National Cancer Institute, Rome, Italy

2) Hematology, Regina Elena National Cancer Institute, Rome, Italy

3) Clinical Pathology, Cytogenetic Unit, Regina Elena National Cancer Institute, Rome, Italy

4) Clinical Pathology, Regina Elena National Cancer Institute, Rome, Italy

Background and purpose of the study- The current diagnostic approach in myelodisplasia includes Peripheral Blood (PB) film and Bone Marrow (BM) aspirate, morphological examination as well as classic cytogenetic and/or FISH BM analysis. The role of BM biopsy is not fully established. Moreover, the diagnostic value of PB flow cytometry immunophenotyping in splenomegalic / myelodisplastic patients has to be evaluated.

Results- In our series of 63 bone marrow biopsies for pancytopenia and suspected myelodisplasia (form August 2001 to April 2008) a diagnosis of BM infiltration by previously occult lymphoma was done in 6 cases. All lymphoma patients were splenomegalic. Five cases were B-cell lymphomas (3 DLBL, 1 Follicular Lymphoma, 1 Primary Splenic Extranodal Marginal Zone Lymphoma ) whereas in 1 case a diagnosis of Peripheral T-cell Lymphoma (PTCL) was done. No cytogenetic aberrations were found in $4 / 6$ cases evaluated on the BM. In the PTCL a splenectomy was performed subsequently due to the splenic size $(24 \times 10 \times 8 \mathrm{~cm}, 1600 \mathrm{gm})$; the flow cytometryc analysis of the splenic lymphocytes identified as prevalent a CD4 positive population. A biclonal rearrangement of the gamma chain gene of TCR was established at the Dept. of Pathology of the Würzburg Institute.

In further 3 patients splenomegalic / non myelodisplastic a diagnosis of Hairy Cell Leukaemia (HCL) was done by flow cytometry PB immunophenotyping, and confirmed by BM core biopsy. The minimal residual disease (MRD) evaluation was done by PB flow cytometry in all the HCL cases after treatment.

Conclusions- The BM biopsy should be considered in the management of myelodisplastic / splenomegalic patients. In our experience an occult lymphoma could was identified in 6/63 cases. Moreover, flow cytometry PB immunophenotyping was diagnostic in all the HCL cases, splenomegalic / non myelodisplastic, diagnosed at our Institute in these years. A significant correlation with the BM trephine biopsy was found in all the cases. 


\section{LS 147}

\section{HDM4 IS OVEREXPRESSED IN MANTLE CELL LYMPHOMA AND ITS INHIBITION INDUCES THE EXPRESSION OF P21}

M Liang, X Han, M Fernandez, M Nguyen, GZ Rassidakis, I Drakos, LJ Medeiros and *CE Bueso-Ramos

The University of Texas MD Anderson Cancer Center, Houston, TX, United States.

Background: Alterations of human homolog of murine double minute 2 (HDM2)/HDM4 contribute to the tumor development and progression. HDM4 inhibits p53-mediated transcriptional activation and synergistically promotes $\mathrm{p} 53$ and $\mathrm{p} 21$ degradation with HDM2. The role of HDM4 in lymphomas is unclear. We assessed HDM4 expression in mantle cell lymphoma (MCL).

Result: HDM4 expression was assessed using immunohistochemical techniques and routinely fixed and processed tissue sections of reactive lymph nodes and MCL. HDM4 nuclear expression was observed in 18 of 19 (95\%) MCL, but was negative in mantle zone Bcells of reactive lymph nodes $(n=19)(p<0.01)$. HDM4 overexpression was confirmed by quantitative real-time polymerase chain reaction (RT-PCR) in $4 \mathrm{MCL}$ cell lines and $6 \mathrm{MCL}$ tumors and both splicing variants, HDM4-S (containing only p53 binding domain), and HDM4-full-length were increased compared with normal CD19+ Bcells. The HDM4-S to HDM4-full-length ratio was also increased in MCL compared with normal CD19+ B-cells $(\mathrm{p}<0.05)$. Using small interfering RNA (siRNA) to inhibit HDM4 in two MCL cell lines, SP53 (wild type p53) and Mino (mutant p53), p21 protein levels were induced as shown by Western blotting. Immunocytochemical analysis also showed increased p21 and active Caspase-3-positive cells (see table).

\begin{tabular}{ccccc}
\hline & P21 & & \multicolumn{2}{c}{ Active Caspase-3 } \\
\hline & $\begin{array}{c}\text { siRNA } \\
\text { control }\end{array}$ & $\begin{array}{c}\text { siRNA } \\
\text { HDM4 }\end{array}$ & $\begin{array}{c}\text { siRNA } \\
\text { control }\end{array}$ & $\begin{array}{c}\text { siRNA } \\
\text { HDM4 }\end{array}$ \\
\hline $\begin{array}{c}\text { Mino } \\
(\%)\end{array}$ & 5 & 12 & 7 & 10 \\
$\begin{array}{c}\text { SP53 } \\
(\%)\end{array}$ & 15 & 42 & 9 & 15 \\
\hline
\end{tabular}

Conclusion: Aberrant expression of the HDM4-S variant in MCL cell lines and tumors suggests that HDM4 may have a pathogenetic role in this neoplasm. HDM4 is a potential therapeutic target in MCL.

\section{LS 148}

\section{INDOLENT CD8-POSITIVE LYMPHOID PROLIFERATION OF THE FACE. A DISTINCT PRIMARY CUTANEOUS T-CELL LYMPHOMA?}

Tony Petrella, Eve Maubec, Pascale Cornillet-Lefebvre, Rein Willemze, Eduardo Marinho Jean-Luc Benhamou, Patty Jansen, Chalid Assaf, Alistair Robson and Florent Grange.
The authors report six cases of cutaneous lymphoproliferation unusual by their histology and their clinical presentation. Each presented with a history of a slow growing nodule on the ear or nose. Despite the indolent clinical evolution, the histology suggested a high-grade lymphoma. All lesions consisted of a dense, diffuse proliferation of monomorphous medium-sized T-cells throughout the dermis and subcutis. There was no epidermotropism and a grenz zone was clearly present in each case. The tumour cells displayed irregular blast-like nuclei, with small nucleoli and clear chromatin and had a CD3+, CD8 +, CD4-, TiA1+, granzyme B- immunophenotype with a loss of other T-cell antigens. The five cases with available material for PCR studies displayed a monoclonal T-cell rearrangement of the T-cell receptor gamma chain. These cases do not correspond to a recognised cutaneous T-cell lymphoma as described in the recent WHO/EORTC classification. The apparent striking propensity for the face (ear and nose) suggests that they might represent a specific entity. Further cases are needed to confirm this hypothesis. It is important for such indolent lesions to be known in order to avoid over treatment.

\section{LS 149}

\section{p65 ACTIVITY AND ZAP-70 STATUS PREDICT THE SENSITIVITY OF CLL CELLS TO A NEW AND SELECTIVE IKK INHIBITOR}

Mónica López-Guerra ${ }^{\text {Ú }}$ BS, Gaël Roué PhD, Patricia Pérez-Galán $\mathrm{PhD}$, Roberto Alonso $\mathrm{PhD}$, Neus Villamor MD, Emili Montserrat $\mathrm{MD}$, Elias Campo MD and Dolors Colomer $\mathrm{PhD}$

Hematopathology Unit, Department of Pathology and Hematology, Hospital Clínic, Institut d'Investigacions Biomèdiques August Pi i Sunyer (IDIBAPS), University of Barcelona, Barcelona, Spain

Background: Chronic lymphocytic leukemia (CLL) is a clinical entity derived from the monoclonal expansion of long-life B-lymphocytes with deregulated proliferation. Constitutive activation of the NF-KB pathway has been implicated in the pathogenesis of this malignancy. The NF- $\mathrm{kB}$ signaling pathway plays a crucial role in tumorigenesis by its capacity to modulate apoptosis and cell survival, as well as proliferation, inflammation, tumor metastasis and angiogenesis.

Purpose of the study: In the present work, we have analyzed the in vitro antitumoral effect of a new and selective IKK inhibitor, BMS345541, in 34 primary CLL cells.

Results: BMS-345541 selectively induced apoptosis in CLL cells in the low micromolar range, irrespective of p53 or ATM status. Noteworthy, the ZAP-70high group was significantly more sensitive to BMS-345541 than the ZAP-70low group, in correlation with high levels of p65 phosphorylation and DNA binding activity. Following NF- $\mathrm{KB}$ inhibition, BMS-345541 led to induction of the mitochondrial apoptotic pathway and activation of both caspase-dependent and independent factors. Moreover, apoptosis induced by this inhibitor was accompanied by downregulation of several antiapoptotic NF- $k B$ target genes, including both Bcl-2 family members (Bcl- $\mathrm{X}_{\mathrm{L}}, \mathrm{Mcl}-1$, Bcl-2 and Bfl-1/A1) and apoptotic endogenous inhibitors (cFLIP, XIAP, cIAP2 and survivin). In this context, we demonstrated a strong synergism between BMS-345541 and conventional chemotherapeutics such as mitoxantrone and dexamethasone, as well as with new promising drugs such as the BH3-mimetic GX15-070/Obatoclax or the anti-TRAIL-R1 monoclonal antibody mapatumumab. 
Conclusion: Taken together, these data confirm that NF- $k B$ is a relevant target in CLL and indicate that inhibitors of IKK, alone or in combination, represent a promising therapeutic strategy for the treatment of CLL patients, especially for the high-risk prognosis group with ZAP-70 high expression.

\section{LS 150}

\section{EBV ASSOCIATED EXTRA-NODAL NK CELL LYMPHOMA NASAL TYPE OF THE BREAST IN BEHCET DISEASE: A CASE REPORT}

*L.Jabri, A. Benkirane, S. Benayad, M. Karkouri, S. Zamiati

Department of Pathology

University Hospital Ibn Rochd, Casablanca, Morocco

Primary non Hodgkin lymphoma of the breast is uncommon. Most are of B-cell phenotype, with only rare cases showing a T-cell phenotype, NK-cell lymphomas are extremely rare, only 2 cases are reported so far in the literature. The association of Behcet's disease with malignancies has rarely been reported. Only 6 cases of lymphoma have been reported, of whom one is a nasal NK-cell lymphoma. We report a case of NK-cell lymphoma extranodal nasal type of the breast

It is a 20 year-old woman followed up for Behcet disease under immusuppressive treatment for 4 years. She presented with a mammary nodule of $3 \mathrm{~cm}$. The biopsy of the lesion revealed the infiltration of medium to large sized lymphoma cells with necrosis and angiocentric pattern. The cells were positive for cytoplasmic CD3, CD56, CD8, TiA1 and Granzyme B, negative for surface CD3. Analysis of T-cell receptor fails to show clonal rearrangement. EBV studies show clonal episomal integration of EBV (EBER-1 positive). She underwent chemotherapy and radiotherapy and achieved complete remission.

To our Knowledge this case report is the first to describe NK-cell lymphoma of breast arising in Behcet disease.

\section{LS 151}

\section{APOPTOSIS, MACROPHAGE INFILTRATION AND REGULATORY T-CELLS IN DIFFUSE LARGE B-CELL LYMPHOMA}

*Tamasin N Doig ${ }^{(1)}$, Chris Gregory ${ }^{(1)}$, Donald Dunbar ${ }^{(1)}$ and John R Goodlad $^{(2)}$.

(1) Centre for Inflammation Research, The Queen's Medical Research Institute, University of Edinburgh, 47 Little France Crescent, Edinburgh, EH16 4TJ, UK.

(2) Department of Pathology, Western General Hospital, Crewe Road South, Edinburgh, EH4 2XU, UK.

Background: The presence of apoptotic cells in tumours may exert effects on tumour associated macrophages for the benefit of the tumour by both modulation of the immune response to tumour and provision of growth or survival signals. One mechanism by which the immune response to tumour may be controlled is by the action of regulatory T-cells. Using pre-treatment human biopsy material of Diffuse Large B-cell Lymphoma (DLBCL) we examined whether a relationship exists between tumour cell apoptosis, proliferation, macrophage infiltration and presence of regulatory T-cells.

Purpose: To establish whether the presence of FOXP3+ regulatory Tcells is dependent on the relationship between apoptotic lymphoma cells and macrophages, a tissue microarray containing 82 cases of DLBCL was analysed for proliferation, apoptosis, macrophage infiltration and presence of regulatory T-cells by immunohistochemical expression of ki67, activated caspase-3, CD68 and FOXP3 respectively and indices of positive cells calculated for each.

Results: The percentage of FOXP3+ regulatory T-cells in areas of tumour varied from 0 to $18 \%$ of total cells, with a median value of $2.1 \%$ of total cells. Median values for proliferation, apoptosis and macrophage infiltration were $70 \%, 1.5 \%$ and $11 \%$ of total cells respectively. There was no significant difference in percentage of FOXP3+ cells, proliferation, apoptosis or extent of macrophage infiltration between DLBCL of germinal centre and non-germinal centre type. While there were weak associations between apoptosis, proliferation and macrophage infiltration, there were no associations between the percentage of FOXP3+ cells and indices of either proliferation, apoptosis or macrophage infiltration.

Conclusion: In DLBCL a weak relationship exists between presence of apoptotic cells, macrophage infiltration and proliferation, but no such relationship appears to exist with FOXP3+ regulatory T-cells. Thus, in this series there is no evidence that apoptotic tumour cells exert an influence on the immune response via FOXP3+ regulatory T-cells.

\section{LS 152}

\section{FOLLICULAR LYMPHOMA IS CHARACTERIZED BY THE UPREGULATION OF SEVERAL MICRORNAS IN COMPARISON TO NORMAL FOLLICULAR B-CELL POPULATIONS}

Malpeli $\mathrm{G}^{* \wedge}$, Barbi $\mathrm{S}^{\wedge}$, Bertolaso $\mathrm{A}^{\wedge}$, Zamò $\mathrm{A}^{\wedge}$, Zupo $\mathrm{S}^{0}$, Amato $\mathrm{E}^{\wedge}$, Mosna $\mathrm{F}^{+}$, Krampera $\mathrm{M}^{+}$, Croce $\mathrm{C}^{\S}$ and Scarpa $\mathrm{A}^{\wedge}$

${ }^{\wedge}$ Department of Pathology, University of Verona, Verona, Italy

${ }^{0}$ Lymphoproliferative Diseases Unit, National Institute for Cancer Research, Genova, Italy

${ }^{+}$Department of Clinic and Experimental Medicine, University of Verona, Verona, Italy

${ }^{\S}$ Department of Molecular Virology, Immunology and Medical Genetics and Comprehensive Cancer Center, The Ohio State University, Columbus, USA

Background. Follicular lymphoma (FL) is a neoplastic proliferation of B-cells, putatively derived from the follicle center B-cells. FL displays peculiar cytogenetic abnormalities responsible of early events, but yet unidentified secondary alterations are needed for progression to malignancy.

Purpouse. microRNAs are known to participate to normal B-cell development and lymphomagenesis but scarce data regard their involvement in FL.

Results. To evaluate this aspect, we performed the expression analysis of microRNAs by microarray in $25 \mathrm{FL}$ at different clinical stages and compared it to pure normal naïve, germinal center and post-germinal center B-cell populations. As already reported in the literature, proliferating normal germinal center B-cells are characterized by the downregulation of mir-150, but our data indicated other microRNAs varied during the normal B-cell development. Moreover, we identified 
26 microRNAs significantly differentially expressed between FL and normal B-cells Most of expression differences between FL and normal B-cells regarded higher rather than lower expression in FL. All FL showed a recurrent pattern of microRNA expression alterations and the expression of a single microRNA was a promising marker for identification of FL cells. Expression data were validated by quantitative PCR and further confirmed by in situ hybridization with LNA probes on paraffin-embedded FL.

Conclusions. Our findings indicated that expression of many microRNAs is deregulated in FL and some of them could be involved in the pathogenetic mechanism of FL.

\section{LS 153}

\section{E2F1 AND APOPTOSIS IN HODGKIN'S LYMPHOMA}

I. Doussis-Anagnostopoulou ${ }^{1 *}$, E. Georgiadi ${ }^{1}$, T.P. Vassilakopoulos ${ }^{2}$, M.C. Kyrtsonis ${ }^{2}$, M.K. Angelopoulou ${ }^{2}$, G.A. Pangalis ${ }^{2}$, V. Gorgoulis ${ }^{1}$, C. Kittas ${ }^{1}$.

${ }^{1}$ Laboratory of Histology and Embryology and ${ }^{2}$ Department of Haematology, "Laikon" General Hospital, National and Kapodistrian University of Athens, Greece.

Background: E2Fs is a family of transcription factors critical in the progression of cell cycle. E2F1, the first and best characterized member, is a transcriptional activator, due to its ability to induce Sphase entry in quiescent cells, that is mainly dependent on the phosphorylation state of the retinoblastoma protein, pRb. Furthermore, E2F1 can induce apoptosis in both a p53-dependent and p53independent ways. Its interchanging roles, either as an oncogenic or as a tumour suppressive molecule, makes E2F1 an interesting participant in tumorigenesis. Although its role has been investigated in various tumours, information in lymphomas is limited.

Purpose of the study: We have previously established the expression of E2F1 in Hodgkin's lymphoma and demonstrated no relationship neither with proliferation nor with morphological apoptosis. In the present study, we expand our series studying apoptosis both morphologically and using the Tdt-mediated dUTP nick end labeling (Tunel) method in 84 cases of HL. Additionally, the expression of p53 protein was also investigated immunohistochemically. Our results were correlated with E2F1 immunohistochemical expression, clinicopathological parameters and patient prognosis.

Results: Morphological apoptosis ranged from 2-37 (median $10.1 \%$ ), while when evaluated by the Tunel method it ranged from 3-59 (median 11.3\%). P53 expression ranged between 0-96 (median $32.9 \%$ ). E2F1 showed a positive correlation with p53 expression (Spearman's Rho $=0.31, \mathrm{p}=0.01$ ), while $\mathrm{p} 53$ was also positively correlated with MIB1 expression (Spearman's Rho $=0.268, \mathrm{p}=$ 0.028 ). No other statistically significant correlation was found. The expression of E2F1, p53 and all apoptotic indices were similar both in patients who had failed primary chemotherapy and those who were in sustained first complete remission at the time of the analysis.
Conclusion: In the context of Hodgkin' lymphoma, E2F1 does not seem to be directly associated neither with proliferation nor with apoptosis, evaluated morphologically or by Tunel. These results are intriguing, in view of the central role of E2F1 in cell cycle progression

\section{LS 154}

\section{LYMPH NODE HISTOMORPHOLOGY DOES NOT CORRELATE WITH ZAP-70 EXPRESSION IN CLL/SLL}

Tomas Balharek, Lukas Plank, Peter Szepe, Juraj Marcinek, Zuzana Kviatkovska, Martina Barthova

Department of Pathology and National Consultation Centre of Haematopathology, Comenius University, Jessenius Faculty of Medicine and Faculty Hospital, Martin, Slovak Republic

Background: Chronic lymphocytic leukaemia/small lymphocytic lymphoma (CLL/SLL) typically presents as diffuse proliferation with pseudofollicular growth centres (PFCs) completely effacing normal lymph node (LN) architecture. Only small subset of CLL/SLL cases shows a partial LN involvement with preserved reactive lymphatic follicles, so-called interfollicular growth pattern. Number and size of PFCs vary from case to case, but there is no evidence that identification of various patterns of PFCs morphology in LN biopsies is of biological relevance. Because ZAP-70 is a strong prognostic indicator defining cases with potentially aggressive behaviour, in this study we analyzed relation between histomorphology of LN infiltrates and ZAP-70 expression.

Methods: We analyzed LN biopsy specimens in 45 CLL/SLL cases diagnosed according to principles of WHO classification (2001). LN involvement pattern (total effacement versus interfollicular growth) and size of PFCs (,typical“ versus prominent occupying $>30 \%$ of LN area) were analyzed. ZAP-70 expression was detected immunohistochemically (IHC) in paraffin-embedded tissue sections using ZAP70 antibody (clone 2F3.2, Upstate). ZAP-70 expression was scored as negative, weak positive $(1+)$ or strong positive $(2+)$.

Results: Interfollicular LN involvement with residual LF was recognized in 9 cases $(20 \%)$. Analyzed types of LN involvement did not differ in presence of ,typical“ or prominent PFCs. Prominent PFCs were present in $33 \%$ of interfollicular CLL/SLL and in $44 \%$ of cases with complete infiltration. Cases with and without interfollicular pattern also did not show significant differences of ZAP-70 expression. The proportion of ZAP-70 2+, 1+ and negative cases was relatively equal in both groups $(44,5 \% / 33,5 \% / 22 \%$ versus $47 \% / 19,5 \% / 33,5 \%)$.

Conclusions: We did not identify any significant relation between type of LN involvement, morphology of PFCs and ZAP-70 expression. Biological and prognostic sense of interfollicular involvement and prominent PFCs in LN specimens remains unclear. Supported by grants of Slovak Ministry of Health Nr. 2005/12MFN-04 and VEGA Nr. 1/4285/07. 


\section{LS 155}

\section{DELINEATION OF MATURE AGGRESSIVE B-CELL LYMPHOMA BY MICRORNA EXPRESSION PROFILING}

${ }^{*}$ Dido Lenze ${ }^{1}$, Michael Hummel ${ }^{1}$, Korinna Jöhrens ${ }^{3}$, Eleonora Leucci $^{2}$, Anna Onnis ${ }^{2}$, Stefano Volinia ${ }^{4}$, Chang-Gong Liu ${ }^{4}$, Carlo Croce $^{4}$, Lorenzo Leoncini ${ }^{2}$ and Harald Stein ${ }^{1}$

\author{
${ }^{1}$ Institute of Pathology, Campus Benjamin Franklin, Charité-Univer- \\ sitätsmedizin Berlin, Germany \\ ${ }^{2}$ Department of Human Pathology and Oncology, University of Siena, \\ Italy \\ ${ }^{3}$ Institute of Pathology, Campus Mitte, Charité-Universitätsmedizin \\ Berlin, Germany \\ ${ }^{4}$ Department of Molecular Virology, Immunology and Medical \\ Genetics, Ohio State University, USA
}

Diffuse large B-cell lymphoma (DLBCL) and Burkitt lymphoma (BL) show a heterogeneous clinical outcome. Conventional methods (e.g. histology, immunohistochemistry, FISH analysis) are not sufficient to reliably identify clinical subgroups within DLBCL or to delineate DLBCL from BL. By gene expression profiling molecular subgroups have been described which subdivide DLBCL into two clinical groups (ABC- and GCB-DLBCL) with adverse outcome. Furthermore, BL can be accurately distinguished from DLBCL by robust expression signatures. Despite this progress a significant proportion of patients cannot unequivocally be assigned to a certain clinical subgroup. In search for additional molecular criteria with discriminating power, the analysis of microRNA expression appears to be a promising approach. RNA was extracted from paraffin specimens of 450 DLBCL patients enrolled in the German RICOVER-60 trial, which were treated with CHOP or with R-CHOP, respectively. A total of 70 endemic BL (eBL) and sporadic BL (sBL) paraffin samples was additionally included in this study. ANOVA, hierarchical and partitioning clustering methods were employed in order to detect differentially expressed microRNAs and to correlate the corresponding subgroups with the clinical outcome. Several microRNAs were found to be differentially expressed between DLBCL and BL, whereas only few microRNAs distinguish between ABC- and GCB-types of DLBCL as defined by immunohistochemistry. In addition, microRNAs were able to create own subgroups independent from previous molecular or histological classifications. The correlation of these microRNA subgroups with clinical data revealed discrimination into prognosis related groups. Although eBL and sBL are indistinguishable based on established molecular expression signatures, some microRNAs are differentially expressed between both types of BL.

Our data show that microRNA profiles provide a further important tool to distinguish BL from DLBCL, which is also applicable for RNA from paraffin-embedded tissues. The detection of new prognostic molecular groups within DLBCL might lead to an alternative diagnostic approach which appears to be valid also in patients treated with R-CHOP. Furthermore, some microRNAs are differentially expressed between sBL and eBL supporting the concept that they represent different lymphoma subtypes of BL.
LS 156

\section{CLINICOPATHOLOGICAL FEATURES AND MICROSATELLITE INSTABILITY (MSI) STATUS OF LYMPHOPROLIFERATIVE DISORDERS (LPD) IN INFLAMMATORY BOWEL DISEASE (IBD) FROM THE FRENCH COHORT CESAME.}

P. Cuillière-Dartigues (1), B. Fabiani (1), JF. Fléjou (1), A. Duval (2), L. Beaugerie (3), N. Brousse (4).

(1) Pathology, (3) Gastroenterology, Hôpital Saint-Antoine, APHP, Paris, France.

(2) Inserm U893, Paris, France.

(4) Pathology, Hôpital Necker, APHP, Paris, France.

Background: Chronic inflammation and immunosuppression enhance incidence of mismatch repair (MMR) deficient tumors. We analyzed the clinicopathological features and evaluated the MSI status of LPD developing in IBD.

Methods: Among 20,802 IBD patients, 22 cases of LPD were reviewed and tested for Epstein Barr Virus (EBV). MSI was evaluated in $13 / 21$ LPD, by using a PCR-based molecular assay and IHC of hMLH1, hMSH2 and hMSH6 MMR proteins.

Results: 17 Crohn disease, 4 ulcerative colitis, 1 indeterminate colitis were included. Median age at diagnosis of LPD was 56 years (19-79), with 13 males and 9 females. Primary site of involvement was extranodal (15): gastrointestinal tract (8), skin (2), liver (2), central nervous system (1), bone marrow (1), retroperitoneum (1). Histopathological examination showed 2 polymorphic LPD, 10 monomorphic LPD including 9 diffuse large B-cell lymphoma (DLBCL) and 1 anaplastic large T-cell lymphoma (ALCL-T), 1 follicular lymphoma, 2 Hodgkin lymphomas (HL). The bone marrow case corresponded to an unclassified small B-cell proliferation. 9/13 cases (69\%) tested for EBV were positive: $100 \%$ of B-cell polymorphic LPD and HL, and 5/ 8 DLBCL (62\%). The ALCL-T was EBV-. The 6 patients naive for immunosuppressive drug (IS) had follicular lymphoma (3), DLBCL (2), angioimmunoblastic T-cell lymphoma (1) solely EBV+. None of LPD tested was MMR deficient.

Conclusion: IBD-related LPD belong to the heterogeneous group of immunodeficiency-associated lymphoproliferative disorders by sharing several clinicopathologic features: occurrence in patients receiving IS, frequent extra nodal involvement mainly GI tract in the damaged segment by aggressive B-cell proliferation associated with EBV.

Finally, we failed to show a MSI in our limited series of LPD arising in IBD, unlikely as we demonstrated previously in HIV and posttransplantated patients $(*)$. More cases and especially T-cell neoplasms are needed to confirm hypothesis that IS favours expansion of MMR deficient clones (**).

* Duval et al, PNAS 2004.

** Offman et al, Blood 2004 


\section{LS 157}

\section{EXPRESSION PATTERN OF XBP-1S IN HUMAN B CELL LYMPHOMAS}

*Maestre $\mathbf{L}^{\mathbf{1}}$, Cañamero $\mathbf{M}^{\mathbf{2}}$, Ramos $\mathbf{R}^{1}$, Baena $\mathbf{S}^{1}$, MontesMoreno $S^{3}$, Tooze $R^{4}$ and Roncador $G^{1}$

${ }^{1}$ Monoclonal Antibodies Unit, Biotechnology Programme, Spanish National Cancer Centre (CNIO), Madrid, Spain.

${ }^{2}$ Comparative Pathology Unit, Biotechnology Programme, CNIO, Madrid, Spain.

${ }^{3}$ Lymphoma Group, Molecular Pathology Programme, CNIO, Madrid, Spain.

${ }^{4}$ Leeds Institute of Molecular Medicine. University of Leeds, Leeds, UK.

Background: XBP-1 (X-box-binding protein 1) is a member of the CREB/ATF (cyclic AMP response element binding protein/activating transcription factor) family of transcription factors. It has been shown to be essential for plasma cell differentiation and for immunoglobulin synthesis by B lymphocytes.

Purpose of the study: Using tissue-microarrays (TMA) and Western Blotting analysis, we have investigated the expression of XBP-1s protein in a large series of both normal and neoplastic lymphoid tissues using a novel specific monoclonal antibody reactive on paraffin-embedded tissues.

Results: XBP-1s protein was expressed in the nucleus by all plasma cells, plasma cell-derived neoplasms and lymphomas with plasma cell differentiation (myelomas/plasmacytomas, lymphoplasmacytic lymphomas). Among diffuse large B cell lymphomas (DLBCL) cases only plasmablastic subtype showed XBP-1s expression, with different signal intensities (weak and strong), in a high percentage of cases. A more extensive immunohistochemical analysis of these cases, using CD138, PRDM1/Blimp-1, PAX5, CD20 monoclonal antibodies, was performed. Different patterns of expression of the studied B cell markers were found depending on the intensity of XBP-1 expression. Follicular lymphoma, mantle cell lymphoma, chronic lymphocytic leukaemia, no- plasmablastic DLBCL and T cell lymphomas cases were mainly negative.

Conclusion: We describe a new monoclonal antibody that may be of use for the diagnostic pathologists in the identification of plasma cell differentiation in different $\mathrm{B}$ cell lymphomas as well as normal plasma cells in routinely processed tissue samples. Due to its specific associations with other B cell expression markers, this new antibody can also be used to achieve a better understanding of the pathogenic role of XBP-1s in malignant diseases.
LS 158

\section{PERSISTENT ABERRANT IMMUNOPHENOTYPE AND MONOCLONALITY OF INTRAEPITHELIAL T-LYMPHOCYTES ARE FEATURES OF REFRACTORY COELIAC DISEASE AND THE PRESENCE OF BOTH ABNORMALITIES IS ASSOCIATED WITH CASES THAT EVOLVE TO ENTEROPATHY TYPE T-CELL LYMPHOMA}

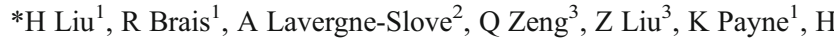
$\mathrm{Ye}^{3}, \mathrm{~J}_{\text {Carreras }}{ }^{3}, \mathrm{Y}$ Huang ${ }^{3}, \mathrm{CM}$ Bacon $^{3}, \mathrm{~V} \mathrm{Save}^{1}, \mathrm{~L}_{\text {Venkatraman }}{ }^{4}$, PG Isaacson ${ }^{5}$, J Woodward ${ }^{6}$ and $\mathrm{M}-\mathrm{Q} \mathrm{Du}^{3}$.

${ }^{1}$ Dept of Histopathology and ${ }^{6}$ Dept of Gastroenterology, Addenbrooke's Hospital, Cambridge, UK; ${ }^{2}$ Anatomie Pathologique, Hôpital Lariboisière, Paris, France; ${ }^{3}$ Dept of Pathology, University of Cambridge, Cambridge, UK; ${ }^{4}$ Dept of Histopathology, Royal Victoria Hospital, Belfast, UK; ${ }^{5}$ Dept of Histopathology, University College London Hospitals, London, UK

Background: Patients with refractory coeliac disease (RCD) have an increased risk for development of enteropathy-type T-cell lymphoma (ETL) and early diagnosis of both conditions is difficult. Although abnormal immunophenotype and monoclonality of intraepithelial $\mathrm{T}$ lymphocytes (IELs) are associated with RCD, their value in RCD diagnosis and ETL surveillance remains to be investigated.

Design: Multiple consecutive duodenal biopsies from $33 \mathrm{CD}, 7$ suspected RCD, $30 \mathrm{RCD}$ and 19 ETL (including 10 with RCD biopsies) patients were investigated for IEL immunophenotype by CD3/CD8 double immunohistochemistry and clonality by PCR for the rearranged TCR genes using BIOMED-2 protocols.

Result: An aberrant immunophenotype, defined as greater than $40 \%$ of IELs having a $\mathrm{CD}^{+} \mathrm{CD}^{-}$immunophenotype (the mean $+3 \mathrm{SD}$ of the percentage of $\mathrm{CD}^{+} \mathrm{CD}^{-}$IELs in the initial diagnostic biopsies of $20 \mathrm{CD}$ cases), was found in 1/33 (3\%), 26/37 (70\%) and 12/14 (86\%) diagnostic biopsies of $\mathrm{CD}, \mathrm{RCD}$ and ETL, respectively. Monoclonality was seen in $7 / 41(17 \%), 25 / 37(68 \%)$ and $16 / 16(100 \%)$ diagnostic biopsies of $\mathrm{CD}, \mathrm{RCD}$ and ETL, respectively. During follow up, persistent aberrant IEL immunophenotype or progressive increase of $\mathrm{CD}^{+}{ }^{+} \mathrm{CD} 8{ }^{-}$IELs, reaching the cut-off value on the latest biopsy, was found in 19/24 (79\%) RCD cases including 6 with normal IEL immunophenotype at the diagnosis of RCD, and in $4 / 7$ (57\%) suspected RCD. Such persistence of, or progressive increase in, aberrant IELs was not seen in $16 \mathrm{CD}$ cases, although a transient aberrant IEL immunophenotype was found in $2 / 6 \mathrm{CD}$ cases not compliant with a gluten free diet (GFD). Similarly, persistent identical monoclonality or progression from polyclonality to monocloanlity was seen in 18/24 (75\%) RCD and 2/7 suspected RCD, but only in $1 / 9 \mathrm{CD}$ cases not compliant with a GFD and in none of the CD cases compliant with a GFD. Together, persistence of, or progressive increase in aberrant IELs and/or persistent monoclonality or progression from polyclonality to monoclonality were seen in 23 / $24(96 \%)$ RCD, 6/7 suspected RCD but only in 2/9 CD cases not compliant with a GFD. Importantly, 6/12 RCD cases with both persistent aberrant IELs and persistent identical monoclonality 
progressed to ETL, while only 1 of the remaining $12 \mathrm{RCD}$ cases developed the lymphoma.

Conclusion: The appearance of a persistent aberrant IEL immunophenotype and persistent identical monoclonality is a feature of RCD. The presence of both abnormalities is strongly associated with RCD cases that develop ETL. Continuous monitoring of both IEL immunophenotype and clonality is more important than snapshot analysis of single biopsies in the diagnosis and follow up of RCD.

\section{LS 159}

\section{BIOMED-2 PCR ANALYSIS OF IGK GENE REARRANGEMENTS IS MORE SENSITIVE THAN THAT OF IGH GENE REARRANGEMENTS FOR DETECTION OF B-CELL CLONALITY IN FOLLICULAR LYMPHOMAS.}

\author{
*Karen Payne ${ }^{1}$, Yuanxue Huang ${ }^{2}$, Rifat Hamoudi ${ }^{2}$, Chris M Bacon ${ }^{2}$, \\ Penny Wright ${ }^{1}$, John W. Grant ${ }^{1}$, Ming-Qing Du ${ }^{2}$, Hongxiang Liu ${ }^{1}$ \\ ${ }^{1}$ Department of Histopathology, Addenbrooke's Hospital, Cambridge, \\ UK \\ ${ }^{2}$ Department of Pathology, University of Cambridge, Cambridge, UK
}

Background: B-cell clonality analysis in routine haematopathological practice is commonly performed by PCR targeting the IGH genes but this has a high rate of false negativity in germinal centre and postgerminal centre B-cell derived lymphomas that are known to have high rates of somatic hypermutation (SHM) in the rearranged IGH genes. The present study assessed the value of analysis of the rearranged IGK genes as compared with that of the IGH genes in follicular lymphomas (FL) using the BIOMED-2 PCR primers and protocols, and determined the frequencies of SHM in the rearranged IGK and IGH genes.

Design: Clonality analysis was performed on paraffin-embedded tissues of FL from 39 patients. The specimens were assessed for DNA quality followed by IG gene rearrangements using a full set of BIOMED-2 reactions and heteroduplex treatment/polyacrylamide gel electrophoresis. The clonal IGH and IGK PCR products were sequenced and analysed for SHM.

Results: Apart from two cases that yielded PCR products of less than $300 \mathrm{bp}$ in size, the quality of DNA samples from all other cases was adequate for the clonality analysis. With the full set of BIOMED-2 reactions, 36 of $39(92 \%)$ cases, including two with suboptimal DNA quality, were found to be clonal. Clonal IG gene rearrangements were detected by two IGK reactions (VK-JK and $\mathrm{VK} /$ intron-Kde) in 33 cases $(85 \%)$, while three IGH VH-JH reactions $\left(\mathrm{VH}_{\mathrm{FR} 1}-\mathrm{JH}, \mathrm{VH}_{\mathrm{FR} 2}-\mathrm{JH}\right.$ and $\left.\mathrm{VH}_{\mathrm{FR} 3}-\mathrm{JH}\right)$ and the three $\mathrm{VH}-$ $\mathrm{JH}$ reactions plus two DH-JH reactions $\left(\mathrm{DH}_{1-6}-\mathrm{JH}\right.$ and $\left.\mathrm{DH}_{7}-\mathrm{JH}\right)$ detected clonality in only 15 cases $(38 \%)$ and 21 cases $(54 \%)$ respectively. Combined use of the two IGK reactions with one or more of the $\mathrm{VH}_{\mathrm{FR} 1}-\mathrm{JH}, \mathrm{VH}_{\mathrm{FR} 2}-\mathrm{JH}$ and $\mathrm{DH}_{1-6}-\mathrm{JH}$ reactions increased the detection rate to over $90 \%$. Sequence analysis showed a mean mutation rate of $11.0 \%$ for the rearranged IGH genes $(n=11)$ and $5.2 \%$ for the IGK genes $(n=20, P=0.02)$.
Conclusion: BIOMED-2 PCR assays targeting IGK gene rearrangements are more sensitive than those targeting IGH gene rearrangements for Bcell clonality analysis in FL. The high false-negative detection rate of the clonal rearranged IGH genes may result from the presence of frequent SHM in the rearranged IGH genes leading to failure of primer annealing.

\section{LS 160}

HISTOPATHOLOGICAL AND IMMUNOHISTOCHEMICAL

\author{
*Nazan Ozsan ${ }^{1}$, Mine Hekimgil ${ }^{1}$, Taner Akalin ${ }^{1}$, Gulsen Kandiloglu ${ }^{1}$ \\ ${ }^{1}$ Department of Pathology, Ege University Faculty of Medicine, Izmir, \\ Turkey.
}

Cutaneous lymphomas show different clinical presentations, prognoses and geographical incidence. The recent WHO/EORTC classification for cutaneous lymphomas based on clinical, histological, phenotypical and molecular characteristics, aimed to be clinically relevant for the management and treatment of patients with cutaneous lymphomas. The goal of this study is to recognize the T- and B-cell lymphoma distribution among the cases diagnosed with skin presentation, reclassify them based on the recent WHO/EORTC classification and also analyse them in the aspect of some morphological and immunohistochemical features. Forty-five cases diagnosed as lymphoma presenting in the skin, other than mycosis fungoides between 1999-2008 were examined. Among them, 25 of the cases $(55.5 \%)$ were B-cell lymphomas $(22$ diffuse large B-cell lymphoma, 1 marginal zone lymphoma, 1 follicular lymphoma, 1 cutaneous infiltration of acute lymphoblastic lymphoma), and $19(42.2 \%)$ were T-cell lymphomas (6 anaplastic large cell lymphoma, $5 \mathrm{CD} 30+$ cutaneous lymphoproliferative disease, 5 peripheral T-cell lymphoma, unspecified, $2 \mathrm{NK} / \mathrm{T}$ cell lymphoma, nasal type, 1 subcutaneous panniculitis like T-cell lymphoma) and one was Hodgkin lymphoma. Four of the B-cell lymphoma cases were proven to be the secondary involvement; three were diagnosed as diffuse large B-cell lymphoma in nodal biopsies and one was diagnosed as precursor B-cell leukemia/ lymphoblastic lymphoma in the bone marrow biopsy. In two patients, bone marrow involvement was seen, without any biopsyproven systemic involvement of other organs. Among T-cell lymphoma cases, five cases were proven to be secondary involvement; two were anaplastic large cell lymphoma, two were peripheral T-cell lymphoma, unspecified in nodal biopsies, and one was extranodal NK/T cell lymphoma, nasal type. None of the T-cell lymphoma cases, either primary or secondary, showed bone marrow involvement. On comparision of our cases with review of the literature, the predominance of Band T-cell lymphomas are between those reported from Europe and Asia. 
LS 161

\section{BCL-2 REARRANGEMENT AND FOXP1 EXPRESSION PREDICT SURVIVAL IN DIFFUSE LARGE B CELL LYMPHOMAS PATIENTS TREATED WITH R-CHOP. A GELA (LNH98-5) STUDY}

Christiane Copie-Bergman*1; Philippe Gaulard 1; Karen Leroy 1; Jean-Philippe Jais 2 ; Josette Briere 3 ; Maryse Baia1 ; Gilles Andre Salles 4 ; Françoise Berger 5 ; Corinne Haioun 6 ; Herve Tilly 7 ; Jean-François Emile 8 ; Pierre Feugier 9 ; Bertrand Coiffier 4 ; Thierry Jo Molina 10.

${ }^{1}$ Pathology, Hôpital Henri Mondor, AP-HP, Créteil, France; ${ }^{2}$ Statistics, Hôpital Necker, AP-HP, Paris, France; ${ }^{3}$ Pathology, Hôpital SaintLouis, AP-HP, Paris, France; ${ }^{4}$ Hematology, Centre Hospitalier Lyon-Sud, Pierre-Bénite, France; ${ }^{5}$ Pathology, Centre Hospitalier Lyon-Sud, Pierre Bénite, France; ${ }^{6}$ Hematology, Hôpital Henri-Mondor, AP-HP, Créteil, France; ${ }^{7}$ Hematology, Centre Becquerel, Rouen, France; ${ }^{8}$ Pathology, Hôpital Ambroise Paré, AP-HP, Boulogne, France; ${ }^{9}$ Hematology, CHU de Brabois, Vandoeuvre les Nancy, France; ${ }^{10}$ Pathology, Hôtel-Dieu, AP-HP, Paris, France.

Background : Diffuse large B cell lymphomas (DLBCLs) have been classified according to gene expression profiling in two main pronostically subgroups « germinal-center type » $(\mathrm{GC})$ and " nongerminal-center type $»(\mathrm{n}-\mathrm{GC})$ related to the cell of origin $(\mathrm{COO})$. In DLBCL patients treated with R-CHOP, the COO classification based on RNA profiling remained of prognostic value whereas immunohistochemical assessment of $\mathrm{COO}$ according to Hans'algorithm has proved controversial. Our group recently identified a 16 gene RNA signature predicting survivl in elderly patients with DLBCL treated with R-CHOP

Purpose of the study : (1) To evaluate the prognostic value of one of these 16 genes FOXP1 and of the Hans' algorithm using immunohistochemistry on tissue microarrays of 172 patients enrolled in the GELA LNH98-5 trial that compared CHOP and R-CHOP. (2) To determine the clinical impact of BCL2, BCL6 and c-MYC gene rearrangements by interphase fluorescence in situ hybridization (FISH) using split-signal DNA probes (Dako).

Results : The GC/nGC profile did not show a significant prognostic impact on survival in R-CHOP patients . BCL2 rearrangement highly correlated with GC subtype and had a favourable impact on Overall Survival $(\mathrm{OS})(\mathrm{p}=0,05)$ in R-CHOP treated patients whereas BCL6 rearrangement tended to be associated with an unfavourable outcome $(\mathrm{p}=0.07)$. Among 150 patients with evaluable FOXP1 staining, 19 $(13 \%)$ showed strong nuclear reactivity, 86 (57\%) displayed variable expression and $45(30 \%)$ were negative. FOXP1 expression was highly correlated with $n-G C$ subtype $(p=0.0003)$. Interestingly, in a Cox Model incorporating aaIPI (age-ajusted International Prognostic Factor) and FOXP1, both significantly influenced EFS in R-CHOP patients ( $\mathrm{p}=0.0012 ; 0.0446$, respectively).

Conclusion: We showed the prognostic value of BCL2, BCL6 gene rearrangements and FOXP1 expression in DLBCL patients treated with R-CHOP. Our study underlines the major interest of combining FISH with immunohistochemistry to build an histoprognostic index on paraffin embedded tissue.
LS 162

\section{THE SUBCELLULAR SOX11 DISTRIBUTION PATTERN IDENTIFIES SUBSETS OF MANTLE CELL LYMPHOMA: CORRELATION TO OVERALL SURVIVAL.}

Wang, X., Asplund, C., Porwit, A., Flygare, J. Smith, C.I.E., Christensson, B. and Sander, B.*

Karolinska Institutet and Karolinska University Hospital Stockholm, Sweden

Background: Most mantle cell lymphomas (MCL) over-express cyclin $\mathrm{D} 1$ as a result of the $\mathrm{t}(11 ; 14)(\mathrm{q} 13 ; \mathrm{q} 33)$. However, this translocation is not sufficient to induce MCL and other, yet uncharacterized genetic events are needed in order to establish the lymphoma.

Purpose of the study: Oligonucleotide microarray expression analysis was used in order to identify genes differentially expressed in MCL compared to reactive lymphoid tissue.

Summarized description of the results : The neuronal transcription factor Sox11 was significantly overexpressed in MCL compared to reactive lymphoid tissue. By RT-PCR, Western blot and immunohistochemistry, Sox11 was not only detected in MCL, but also weakly expressed in reactive lymphoid tissue and in small lymphocytic lymphoma and follicular lymphoma. In contrast to other lymphomas and reactive lymphoid tissue, most MCL (tumor samples from 48/53 patients) expressed Sox11 in the nucleus. Therefore, nuclear Sox11 is a new tumor marker for MCL. However, in approximately $10 \%$ of MCL (5/53 tumors) Sox 11 was only expressed in the cytoplasm. MCL patients with nonnuclear expression of Sox11 in their tumors had a significantly shorter survival compared to MCL with Sox11 expression in the nucleus.

Conclusion: The results of this explorative study suggest that expression of Sox11 in MCL is not only a new tumor marker but may also carry information related to the clinical and biological behaviour of MCL.

\section{LS 163}

\section{LOSS OF T CELL IDENTITY AND BY-PASS OF TCR SIGNALLING BY ONCOGENIC TYROSINE KINASE ACTIVITY IN ANAPLASTIC LARGE CELL LYMPHOMA.}

*Ambrogio $\mathrm{C}^{1,2}$, Voena $\mathrm{C}^{1,2}$, Manazza $\mathrm{AD}^{1,2}$, Martinengo $\mathrm{C}^{1}$, Costa $\mathrm{C}^{3}$, Kirchhausen $\mathrm{T}^{4}$, Tondat $\mathrm{F}^{1}$, Hirsch $\mathrm{E}^{3}$, Inghirami $\mathrm{G}^{1,2,5}$ and Chiarle R. ${ }^{1,2}$

${ }^{1}$ Center for Experimental Research and Medical Studies (CERMS), ${ }^{2}$ Department of Biomedical Sciences and Human Oncology and ${ }^{3}$ Department of Clinical and Biological Sciences and Molecular Biotechnology Center (MBC), University of Torino, 10126 Italy, ${ }^{4}$ Department of Cell Biology, Harvard Medical School, Boston, MA 02115-5701, USA, ${ }^{5}$ Department of Pathology and New York Cancer Center, New York University School of Medicine, New York, 10016 USA.

The oncogenic tyrosine kinase Nucleophosmin (NPM)-Anaplastic Lymphoma Kinase (ALK) is expressed after the $t(2 ; 5)$ translocation in the majority of Anaplastic Large Cell Lymphomas (ALCLs), which are clinically characterized by the loss of T cell associated molecules. Here we show that NPM-ALK activity down-modulated the expres- 
sion of the T cell associated molecules ZAP70, LAT and SLP76 both in vitro and in vivo in transgenic mice. NPM-ALK suppressed ZAP70, SLP76 and LAT expression by STAT3 mediated gene methylation as showed by NPM-ALK knock-down through lentiviral inducible shRNA and by NPM-ALK activity inhibition. Despite the lack of T cell associated molecules which mediate TCR signalling, ALCL cells proliferate and display an activated phenotype similar to TCR stimulated T cells. The inhibition of NPM-ALK activity resulted in decreased $\mathrm{T}$ cell lymphoma survival, proliferation and migration together with a loss of anaplastic morphology due to a rearrangement of actin filaments. To this end, NPM-ALK specifically enhanced Rac1 and $\mathrm{Cdc} 42$ activity through the phosphorylation of VAV1, together with a concomitant inhibition of RhoA activity. In vitro, ALCL cells knocked-down for Cdc42 or VAV1 by shRNA showed decreased survival and growth rate and defective motility. Pharmacological inhibition of $\mathrm{Cdc} 42$ activity by secramine resulted in a cell-cycle arrest and apoptosis of ALCL cells. Importantly, the concomitant inhibition of Cdc42 and NPM-ALK kinase by a specific inhibitor acted synergistically to induce apoptosis of ALCL cells. Finally, ALCL cells depleted for Cdc42 by shRNA completely lost their tumorigenicity in a xenograft model in immunodeficient mice and established tumors regressed after the knocked-down of Cdc42. In conclusion, our data indicate a fundamental role of GTPase activation in $\mathrm{ALK}^{+} \mathrm{ALCL}$ growth and maintenance.

\section{LS 164}

\section{HUMAN IMMUNODEFICIENCY VIRUS-ASSOCIATED LYMPHOMAS RECLASSIFICATION WITH TMA-BASED IMMUNOHISTOCHEMISTRY AND MOLECULAR BIOLOGY, ITS ASSOCIATION TO EPSTEIN-BARR VIRUS, HUMAN HERPESVIRUS-8 INFECTION AND EXPRESSION OF NFK-B AS PROGNOSTIC FACTORS}

Montante-Montes de Oca D, Chablé-Montero F, Martínez-Benítez B, Marquina B, Lara C, Angeles-Angeles A, and Lome-Maldonado C.

Department of Pathology. Instituto Nacional de Ciencias Médicas y Nutrición, México City.

Objective: To know the clinical and pathological features of HIVassociated lymphomas, their association to EBV and HHV-8 infection and their expression of NFkB as prognostics factors.

Material and methods: HIV-associated lymphomas were selected from 1985 to 2005. All cases were reclassificated on basis of morphology, TMA-based immunohistochemistry and molecular biology. The clinical features were obtained from the clinical files.

Results: We reviewed $57 \mathrm{HIV}$-positive lymphomas. The average age was 40 years, $95 \%$ were men and $5 \%$ women, $92.9 \%$ were in $\mathrm{C} 3$ stage, and 78 had B symptoms. The most frequent localization was nodal (22 cases), oral cavity, CNS, colon and rectum (6 cases each) and pulmonary ( 5 cases). BM was involved in 13 cases; 31 cases were in clinical stage IVB. Thirty-six cases were reclassificated; 21 cases were excluded due to insufficient material. Twenty-five cases were diffuse large B-cell lymphoma, 6 Burkitt lymphoma (3 with extensive plasmacytoid differentiation), 3 plasmablastic lymphomas (PL) and 2 Hodgkin lymphoma. Twenty-one cases were EBER positive, 19 NFkB positive and 12 both positive. One PL was HHV8-positive. Figure 1 shows the overall survival based in EBV and NF-kB expression.
Conclusions: In this group of lymphomas, we find some differences in prognostic factors, according to the expression of EBER and NFkB. This finding suggests that alternative mechanism could be involved in oncogenic pathways. However, it is mandatory to study more cases to determine the possible relationship between of EBV infection and NFkB pathway as biological and prognostic factors in HIV-associated lymphomas.

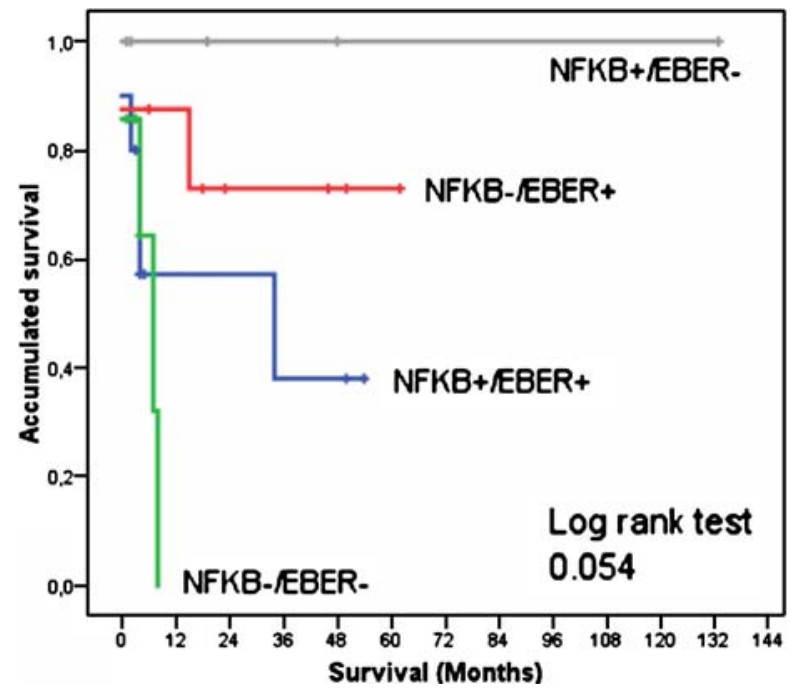

\begin{tabular}{|l|l|l|l|}
\hline $\begin{array}{l}\text { NFkB/EBER } \\
\text { expression }\end{array}$ & $\mathbf{n}$ & $\begin{array}{l}\text { Follow up } \\
\text { (months) }\end{array}$ & $\begin{array}{l}\text { DOD } \\
\text { n (\%) }\end{array}$ \\
\hline NFkB+/EBER- & 5 & $1-133$ & $0(0)$ \\
\hline NFkB-/EBER+ & 8 & $0-62$ & $2(25)$ \\
\hline NFkB+/EBER+ & 10 & $0-54$ & $5(50)$ \\
\hline NFkB-/EBER- & 7 & $0-8$ & $4(57)$ \\
\hline
\end{tabular}

Figure 1. Survival of patients with HIV-associated lymphomas according to NFkB and EBER expression. Double-negative (NFkB-/ EBER-) cases show poor survival followed up for double-positive $(\mathrm{NFkB}+/ \mathrm{EBER}+)$ cases. The cases with one positive marker show better prognosis (Kaplan-Meier curve. Long rank test $\mathrm{p}=0.054$ ). DOD: dead of disease.

\section{LS 165}

COMPARATIVE ANALYSIS OF IGVH GENE REPERTOIRE AND MUTATION PROFILE IN LYMPHOPLASMOCYTIC AND MARGINAL ZONE LYMPHOMAS FROM LYMPH NODES.

Gachard N. ${ }^{1}$, Petit B. ${ }^{2}$, Marfak A. ${ }^{1}$, Soubeyran I. ${ }^{3}$, Coste V. ${ }^{4}$, Parrens M. ${ }^{5}$, Laforet M.P. ${ }^{1}$, Troadec E. ${ }^{1}$, Bordessoule D. ${ }^{6}$, Feuillard J. ${ }^{1}$

${ }^{1}$ Laboratory of Haematology, and UMR CNRS 6101, CHU Dupuytren, Limoges; ${ }^{2}$ Department of Pathology and UMR CNRS 6101, CHU Dupuytren, Limoges; ${ }^{3}$ Department of Pathology and INSERM U916, Institute Bergonié, Bordeaux; ${ }^{4}$ Department of Pathology, Hôpital Gui de Chauliac, Montpellier; ${ }^{5}$ Department of Pathology and Molecular Biology, EA 2406 CHU of Bordeaux; ${ }^{6}$ Department of Haematology and UMR CNRS 6101, Hôpital Dupuytren, Limoges; 
Nodal Marginal Zone lymphomas (NMZL) and lymphoplasmocytic lymphomas (LPL) from lymph nodes (N-LPL) are rare lymphomas which share clinical, morphological and immunohistochemical features that make them difficult to discriminate. Immunoglobulin (Ig) variable heavy chain gene $(\mathrm{IgVH})$ usage and somatic mutation pattern were analyzed to try to clarify their relationship and their pathogenesis.

Nodal frozen samples from 22 patients, 8 N-LPL and 14 NMZL, were studied. B-cell clonality was analysed according to Biomed 2 protocol. Sequence of informative PCR products was analyzed on IMGT, V-BASE and Ig-Blast websites. Antigen driven selection was studied according to Lossos multinomial model. Plasma cell differentiation was evaluated morphologically and based on CD38, CD138 and MUM1/IRF4 expression.

There was no predominance of VH usage, notably for VH3 gene. Diversity of VDJ junction and CDR3 length were similar in N-LPL and NMZL. Although median somatic mutations rate was higher in NLPL $(8 ; 6 \%)$ than in NMZL $(2.8 \%)$, there was no significant difference between the two groups with a mutated threshold of $2 \%$ or of $5 \%$. This could be explained by the presence of two distinct subsets of $\mathrm{N}$ LPL, one highly mutated $(>7 \%)$ and the other unmutated $(<1 \%)$. Distribution of replacement and silent mutations in Complementary Determining Regions and Framework Regions argued for an antigen driven selection process in VH3 positive cases, independently of the diagnosis. Finally, the presence of plasmocytic differentiation was associated with a higher degree of mutation, again independently of the diagnosis.

Altogether, $\mathrm{IgVH}$ repertoire and mutational status suggests that $\mathrm{N}$ LPL and NMZL have a resembling relationship to antigen exposure. A mutated IgVH profile was associated with VH3 segments and/or plasma cell differentiation rather than to the diagnosis of N-MZL versus N-LPL. Our data are also in favor of an antigen driven selection for these lymphomas displaying $\mathrm{VH} 3$ gene.

\section{LS 166}

\section{SURVIVIN EXPRESSION IN AGGRESSIVE B-CELL LYMPHOMA}

Korkolopoulou $\mathrm{P}^{1}$, Angelopoulou $\mathrm{MK}^{2}$, Salpeas $\mathbf{V}^{\mathbf{1 , 2}}$, Galani $\mathrm{Z}^{2}$, Vassilakopoulos $\mathrm{TP}^{2}$, Levidou $\mathrm{G}^{1}$, Anastasopoulou $\mathrm{A}^{2}$, Sachanas $\mathrm{S}^{2}$, Lilakos $\mathrm{K}^{2}$, Papadaki $\mathrm{T}^{3}$, Androulaki $\mathrm{A}^{1}$, Patsouris $\mathrm{E}^{1}$, Pangalis $\mathrm{GA}^{2}$.

(1) $1^{\text {st }}$ Department of Pathology,National and Kapodistrian University of Athens

(2)Department of Hematology, National and Kapodistrian University of Athens

(3)Department of Hematopathology, Evangelismos Hospital, Athens

Background: Survivin, a member of the Inhibitor of Apoptosis Proteins, has recently gained attention as a possible therapeutic target in neoplasia, due to its dual role both as an antiapoptotic protein and a cell cycle regulator. Survivin has been associated with an adverse outcome in several malignancies. However its prognostic significance in diffuse large B-cell lymphoma (DLBCL) is controversial.

Purpose: To investigate Survivin expression and localization, its correlation with clinical and laboratory characteristics and its prognostic significance in DLBCL and follicular lymphoma grade III (FLGIII). Survivin was studied by immunohistochemistry in formalin-fixed paraffin-embedded tissues from patients treated and followed in our Unit.
Results: Among 66 patients [median age 63 years (17-90)], 62\% were males, 39\% had elevated LDH and $42 \%$ clinical stage III/IV. Histologic subtypes were: DLBCL $72 \%$, primary mediastinal LBCL $6 \%$, FLGIII $17 \%$ and transformed low grade lymphoma $4 \%$. The vast majority of patients received R-CHOP. Nuclear expression of Survivin was detected in all patients at a median percentage of $11 \%$ of neoplastic cells $(2-90 \%)$. Cytoplasmic Survivin expression was evident in $38 \%$ of the patients. Levels of nuclear Survivin expression were significantly lower in FLGIII compared to other subtypes $(p=$ 0.007). There was no other significant correlation between nuclear or cytoplasmic survivin expression and baseline patients' characteristics. 5 -year failure free survival was $56 \%$ for all patients. Conventional prognostic factors were valid in this patient population. When FLGIII were excluded, higher nuclear survivin expression tended to correlate with inferior FFS at various cut-offs $(25-50 \%)$, although differences were not statistically significant. Interestingly the presence of any cytoplasmic survivin was associated with superior FFS (5-year rates: 93 vs $50 \%, \mathrm{p}=0.01$ ).

Conclusion: Our preliminary data show that Survivin is expressed in the nucleus of neoplastic cells in aggressive B-cell lymphomas. Moreover its prognostic significance may vary according to its nuclear or cytoplasmic localization.

\section{LS 167}

\section{OVEREXPRESSION OF CYCLIN D1 IS RARE IN AGGRESSIVE B-CELL NON-HODGKIN LYMPHOMAS INCLUDING RICHTER'S TRANSFORMATION CASES}

T Vela-Chávez ${ }^{1,2}, \mathrm{M} \mathrm{Kremer}^{2}, \mathrm{~K} \mathrm{Bink}^{3}, \mathrm{~F} \mathrm{Fend}^{4}$, and L QuintanillaMartínez ${ }^{3}$.

${ }^{1}$ Pathology, Instituto Nacional de Cancerología, Mexico City, Mexico

${ }^{2}$ Pathology, Technical University Munich, Germany.

${ }^{3}$ Pathology, Helmholtz Zentrum Munich, Research for Environmental Health, Germany.

${ }^{4}$ Pathology. University Hospital Tuebingen Eberhard-Karls-University, Germany.

Background: Approximately 5\% of B-cell chronic lymphocytic leukemia (B-CLL) patients develop a secondary lymphoma, usually of diffuse large B-cell type (DLBCL), denominated Richter's transformation (RT). We present an index case of RT with the rare overexpression of cyclin D1 (cycD1), loss of B-cell markers and clonally related to the B-CLL component. The aim of this study was to analyze a large series of DLBCL and RT cases for the expression of cycD1 to know its frequency of expression in aggressive B-NHL other than MCL.

Material and methods: CycD1 (clone SP4, DCS) was performed in a group of 75 cases of B-NHL, including 8 cases of RT and 67 cases of de novo DLBCL. FISH analysis was performed with $t(11 ; 14)$ probe and a cyclin D1 probe. Double staining for cycD1 and CD20 was performed using FITC-labeling and IHC; the former was studied under fluorescence microscope.

Results: Immunohistochemical studies of the index case showed positivity for CD20, CD23, and CD5 in B-CLL biopsy. RT biopsy was reactive for $\mathrm{CD} 45$, Vs38c, Bcl-6, and MiB-1 60\%; B-cell markers were negative. The DLBCL component showed strong positivity for cycD1 in $70 \%$ of the cells. Molecular analysis confirmed identical sequences for FR3 of the IgH. 
Cyclin D1 was weakly positive and in less than $20 \%$ of the tumor cells in $1 / 8$ cases $(12.5 \%)$ of RT and in $10 / 67(14.9 \%)$ cases of de novo DLBCL. Scattered positive cells showed cross-linking of cycD1 and CD20 in all cases (1 RT case and 10 DLBCL). FISH analysis did not reveal amplification or translocation of cycD1 in these cases (including index case).

\section{Conclusions}

1) Overexpression of cycD1 in aggressive B-NHL other than MCL, including RT, is rare, even if the SP4 antibody is used.

2) RT cases with overexpression of cycD1 are not related with translocation or amplification of CCND1 gene, and molecular basis remains to be determined.

\section{LS 168}

\section{THE EXPRESSION OF TWO MICRORNAS DISTINGUISH B-CELL ACUTE LYMPHOBLASTIC LEUKEMIA FROM BONE MARROW CD34-POSITIVE CELLS}

Malpeli $\mathrm{G}^{* \wedge}$, Barbi $\mathrm{S}^{\wedge}$, Bertolaso $\mathrm{A}^{\wedge}$, Mosna $\mathrm{F}^{+}$, Krampera $\mathrm{M}^{+}$, Amato $\mathrm{E}^{\wedge}$, Annunziato $\mathrm{F}^{\star}$, Tabilio $\mathrm{A}^{\star}$, Croce $\mathrm{C}^{\S}$ and Scarpa $\mathrm{A}^{\wedge}$

${ }^{\wedge}$ Department of Pathology, University of Verona, Verona, Italy

${ }^{0}$ Lymphoproliferative Diseases Unit, National Institute for Cancer Research, Genova, Italy

${ }^{+}$Department of Clinical and Experimental Medicine, University of Verona, Verona, Italy

${ }^{*}$ Department of Internal Medicine, University of Firenze, Firenze, Italy

- Department of Clinical and Experimental Medicine, University of Perugia, Perugia, Italy

${ }^{\S}$ Department of Molecular Virology, Immunology and Medical Genetics and Comprehensive Cancer Center, The Ohio State University, Columbus, USA

B-cell acute lymphoblastic leukemia (B-ALL) is characterized by the uncontrolled proliferation and accumulation in the bone marrow of lymphoblasts unable to undergo physiological differentiation. Recently, it was reported that microRNA expression profiles permit to discriminate acute lymphoblastic leukemia from acute myeloid leukemia. In this study, we determined the expression profile of 353 microRNAs by microarray in five primary B-ALL cases, two CD34+ cell samples from healthy bone marrow and four CD19+ Blymphocyte samples from peripheral blood of healthy donors. Microarray expression data were validated by quantitative PCR and in situ hybridization. Our results showed that two and eight of 353 tested microRNAs were expressed with a significant difference between BALL and CD34+ cells, and between B-ALL and CD19+ B-cells, respectively. The validation of expression results by quantitative PCR included 25 additional B-ALL cases. All B-ALL cases were confirmed to show the same difference in expression. Moreover, in situ hybridization by LNA probes confirmed that the two differentially expressed microRNAs are useful markers to highlight B-ALL clones in the presence of CD34+ cells. Our findings add new insights potentially useful for the diagnosis and comprehension of the pathogenetic mechanisms leading to B-ALL.

\section{LS 169}

\section{CHROMOGENIC/FLUORESCENT IN SITU HYBRIDIZATION FOR EVALUTION OF LYMPHOMA}

Steve Chen, M.D., Ph.D. Clarient Diagnostics - Aliso Viejo, California

Dennis P. O’Malley, M.D. Clarient Diagnostics - Aliso Viejo, California

Anselm Hii, M.D. Clarient Diagnostics - Aliso Viejo, California Adam Anthony, B.S. Kaiser Permanente San Francisco

Natalia Mangubat, B.S. Clarient Diagnostics- Aliso Viejo, California Michele Hibbard, Ph.D. Clarient Diagnostics -Aliso Viejo, California Kenneth Bloom, M.D. Clarient Diagnostics - Aliso Viejo, California

Knowledge of the genetic abnormalities of lymphoma has increased and techniques for their detection have advanced. In situ hybridization techniques (ISH) to detect the presence of chromosomal translocations have become fairly routine for diagnostic purposes. Fluorescent ISH (FISH) is most commonly used but is typically performed in the cytogenetics laboratory.

We recently discovered a technique (C/FISH) that modifies a FISH slide, making the signals visible using a standard light microscope. This technique applies antibodies with an attached chromogenic substrate to the fluorophores Texas Red and Fluorescein allowing visualization by light microscopy. All currently available FISH probes using these fluorophores can be evaluated by C/FISH. We evaluated a series of lymphomas in our laboratory to determine the feasibility of this technique for clinical use.

Techniques

Materials and Methods: Individual whole slides and a tissue array composed of 25 tissue samples were evaluated using the chromogenic/ FISH (C/FISH) technique (Dako, Glostrup, Denmark). We analyzed IgH split probes on our lymphoma array. Samples were evaluated by light microscopy by two pathologists (DPO, SC). A normal signal was considered to be two fused signals per cell or split signals less than one probe size apart. All other signals were considered abnormal. Only qualitative results were evaluated, with true "abnormal" results requiring the presence of abnormal signal in the majority of cells.

Results: In our pilot evaluation, we found the following results. $6 / 25$ had no signal or were considered over-digested. $5 / 25$ had results that were equivocal. 9/25 had normal results and 5/25 had abnormal results.

Conclusions: C/FISH may be a viable method for evaluation of chromosomal translocations by light microscopy in lymphoma. We are working on optimizing protease digestion and counterstaining procedures to reduce the failure rate. It is anticipated that by the time of presentation, additional probes and results will be available.

\section{$\underline{\operatorname{LS} 170}$}

TYPE AND MATURATIONAL STATUS OF DENDRITIC CELLS IN CUTANEOUS B-CELL LYMPHOPROLIFERATIVE DISORDERS.

Lesley Christie ${ }^{1}$, Carol McKenzie ${ }^{2}$, Tim Palmer ${ }^{2}$, Lee Baker ${ }^{3}$, John R Goodlad $^{1 *}$

1 Department of Pathology and Neuroscience, University of Dundee, Scotland

2 Department of Pathology, Raigmore Hospital Inverness, Scotland.

3 Department of Surgery and Oncology, University of Dundee, Scotland 
Background: Emerging evidence suggests that cutaneous B-cell lymphoproliferations (CBCLPs) represent a spectrum of disease, with B-cell cutaneous lymphoid hyperplasia (B-CLH) at one end, and overt cutaneous marginal zone lymphoma (CMZL) at the other. Whilst a causative agent can be identified in some cases of CBCLP, the aetiology of the vast majority remains unknown.

Purpose of Study: This study aimed to explore the hypothesis that dendritic cells (DCs) play a central role in the generation and regulation of CBCLPs, by comparing the number, subtype and maturational status of DCs in biopsy specimens showing features of B-CLH $(n=14)$ and CMZL $(n=18)$.

Results: Immunohistochemistry was used to identify Langerhan's cells (Langerin), dermal DCs (DC-SIGN) and plasmacytoid DCs (BDCA2), and to differentiate between mature (DC-LAMP) and immature (CD1a) DCs. FXIIIA was used as a marker of alternatively activated dermal DCs and macrophages. The mean number of positive cells $/ \mathrm{mm}^{2}$ was calculated for each antibody in all cases. The number of plasmacytoid and dermal DCs, and the number of DC-LAMP+ cells was significantly greater in B-CLH than CMZL $(p=0.0319, p=$ $0.0101 \mathrm{p}=0.0421$ respectively). Dermal DCs were present in greater numbers than Langerhan's cells. Although there were fewer FXIIIA+ cells in CMZL than B-CLH, the difference was not significant. Moreover, the relative proportion of FXIIIA+ cells was greater in CMZL than B-CLH.

Conclusion: The results indicate that mature dermal DCs are the dominant cell type in B-CLH. Given that mature DCs function as antigen presenting cells, and are involved in T-cell priming, this finding is consistent with the hypothesis that persistent antigenic stimulation is the cause of such lesions. Conversely, the greater proportion of FXIIIA+ cells in CMZL, leads us to speculate that alternatively activated dendritic histiocytes play a more important role at this end of the CBCLP spectrum, possibly by suppressing the antitumour immune response.

\section{LS 171}

\section{PRIMARY NON-HODGKIN LYMPHOMA OF BONE -CLINICAL AND MORPHOLOGICAL CHARACTERISTICS}

V. Janevska1, , L. Spasevska1, G. Zafirovski2, Z. Trajkovski3, R. Jovanovic1, V. Filipovski1, B. Dukova1, G. Petrusevska1

1. Institute of pathology, Skopje

2. Clinic of orthopedic surgery, University Clinical center-Skopje

3. Institute of radiology, University Clinical center-Skopje, Republic of Macedonia

The aim of the study is to analyze the morphologic features of primary Non-Hodgkin lymphoma of bone.

Material and methods: 15 cases of osseous lymphomas were analyzed using 1. radiographic characteristics and radiological grade of tumor's growth, according to Lodwick; 2. localisation, 3. clinical symptoms, 3. histopathological parameters. The patients were treated with chemotherapy in 15 cases and adjuvant radiation in 9 cases.

The follow up period was 3 to 7 years.

Results: The most of the patients had solitary, permeative or/and moth-eaten metadiaphyseal lytic lesion and an extra osseous softtissue mass on plain radiographs. Six cases had periosteal reactions, and 7 cortical involvement. Femur was the most often involved bone7 cases, than humerus- 4 cases, vertebra- 2 cases and tibia 2 cases . 3 cases had moderate grade, and 20 cases had high radiographic grade of tumor's growth.

All the patients had intermittent bone pain, local swelling and weight loss.

According to the WHO classification of malignant lymphoma, 12 cases were diffuse large B-cell lymphoma, 2 lymphoblastic and 1 Burkit like type lymphoma.

Two of the patients died during the follow up period, 3 of them had recurrence of the disease.

Conclusion: The radiographic features and radiographic grade of tumor's growth were important diagnostic strong factors in primary Non-Hodgkin osseous lymphoma and the most often histological type were diffuse large cell type of lymphoma.

\section{LS 172}

\section{CHARACTERIZATION OF CD4+CD57+ TUMOR- INFILTRATING LYMPHOCYTES IN FOLLICULAR LYMPHOMA}

Thielen C.*, Goval JJ., Gofflot S., Bisig B., Boniver J. and de Leval L.

Department of Pathology, Groupe Interdisciplinaire de Génoprotéomique Appliqué (GIGA)- Research, CHU Sart Tilman, Universty of Liège, Belgium.

Background: Over-expression of the BCL-2 anti-apoptotic gene represents the primary oncogenic mechanism in most follicular lymphomas (FL). In addition, numerous data lend support to the crucial role of the microenvironment in the development and clinical course of FL, especially $\mathrm{T}$ cells and accessory cells. In reactive germinal centers, a subset of CD4 $+\mathrm{T}$ cells coexpressing CD57 $\left(\mathrm{T}_{\mathrm{FH}}\right)$ exerts critical functional help to follicular B cells. Little is known about the involvement of $\mathrm{T}_{\mathrm{FH}}$ in FL. Recent studies have suggested a correlation between higher CD57+ cells count and unfavorable clinicobiologic factors in FL.

Purpose of the study: to provide further characterization of tumorinfiltrating lymphocytes with a $\mathrm{T}_{\mathrm{FH}}$ phenotype, in FL.

Results: By immunohistochemistry on paraffin sections, CD57+ cells were detected in all FL biopsies $(n=24)$, and at a variance with reactive follicles, were distributed in both follicular and extrafollicular zones. A significantly lower density of CD57+ T cells was found in cytological higher-grade lesions (grades III versus I+II, $\mathrm{p}<0.001$ ). Immunophenotypic characterization of the CD4+CD57+ cell suspensions from $17 \mathrm{FL}$ and 6 tonsils was performed by 3 colors flow cytometry. In both tonsils and FL, most CD4+CD57+ cells coexpressed CD45RO and CD28. HLA-DR, CD25, CD26, CD62L, CCR3, CCR7, CXCR4 and CXCR5 showed a similar expression in FL and tonsils. The percentage of $\mathrm{CD} 4+\mathrm{CD} 57+$ cells positive for CD69, CXCR4 and ICOS was significantly lower in FL compared to reactive samples $(80 \%$ vs $91 \%, 51 \%$ vs $73 \%, 41 \%$ vs $68 \%$ respectively) whereas the proportion of CCR5+ cells among CD4+ CD57+ cells was higher in FL (71\% vs 53\%). There was no evident association between grade and immunophenotypic variation.

Conclusions: The present data (1) confirm the presence of CD57+ in FL with a density inversely correlated to cytological grade; (2) show aberrant distribution of CD57+ $\mathrm{T}$ cells outside neoplastic follicles in $\mathrm{FL}$; (3) reveal immunophenotypic modulation of CD4+CD57+ cells in $\mathrm{FL}$, in respect to the expression of chemokine receptors and activation markers, which may account for their altered distribution and likely 
reflect modified functional properties. Collectively these data support that CD57+ cells may be relevant to pathobiology of FL.

\section{LS 173}

\section{EXPRESSION OF SURVIVIN IN NEWLY DIAGNOSED ADVANCED HODGKIN'S LYMPHOMA}

${ }^{*}$ Ljubomir R. Jakovic ${ }^{1}$, Biljana Mihaljevic ${ }^{1}$, Maja Perunicic Jovanovic $^{1}$, Andrija Bogdanovic ${ }^{1}$, Tatjana Terzic ${ }^{1}$, Vesna Cemerikic Martinovic $^{1}$, Darinka Boskovic ${ }^{1}$, Vladimir Bumbasirevic ${ }^{2}$

Institute of Hematology ${ }^{1}$, Clinical Center of Serbia, Belgrade, Serbia; Institute of Histology and Embriology ${ }^{2}$, School of Medicine, University of Belgrade, Serbia

Background: Defective regulation of apoptosis plays important role in carcinogenesis. Survivin, an important member of the inhibitor of apoptosis (IAP) family, is over-expressed in many cancers and is suggested to be prognostic marker in monitoring these patients.

Purpose: The aim of the study was to determine the prognostic value of survivin in newly diagnosed pts with advanced Hodgkin's lymphoma (HL). Their significance was correlated with International Prognostic Score (IPS). It was evaluated regarding the response to treatment and survival period.

Summary: In a cohort of 89 pts with classical HL in advanced stage of the disease (CS IIB-IV), treated with ABVD regimen from 1997 to 2004, we analyzed prognostic relevance of survivin expression determined by immunohistochemistry, at diagnosis. Immunohistochemistry was performed on formalin fixed, paraffin embedded lymph nodes' tissue sections using an indirect immunoperoxidase method and a specific monoclonal antibody following standard procedure. The percentage of survivin positive neoplastic cells was analyzed on 10 different high power microscopy fields (HPF, $\times 400)$. The median follow-up was 7 years (yrs).

The OS rate was $67 \%$ after 7 yrs' follow-up. Survivin expression was found in $88.75 \%$ of cases. Survivin expression had no statistically significant influence on OS and EFS ( $\log$ rank $\mathrm{p}>.05$ ). There was no significant correlation between survivin expression and IPS ( $\log$ rank $p>.05)$.

Conclusion: In pts with advanced classical HL we could not prove the predictive power of survivin.

\section{LS 174}

\section{CLINICOPATHOLOGIC SIGNIFICANCE} AND PROGNOSTIC VALUE OF CHROMOSOMAL IMBALANCES IN DIFFUSE LARGE B-CELL LYMPHOMAS TREATED WITH RITUXIMAB-CONTAINING REGIMENS

Itziar Salaverria ${ }^{1}$, Cristina Royo ${ }^{1}$, Teresa Cardesa $^{1}$, Armando LópezGuillermo $^{2}$, Leonor Arenillas ${ }^{3}$, Silvia Beà ${ }^{1}$, Emili Montserrat ${ }^{2}$, Lluís Colomo $^{1}$, Elías Campo ${ }^{1}$

Departments of ${ }^{1}$ Pathology and ${ }^{2}$ Hematology, Hospital Clinic, University of Barcelona, Barcelona, Spain. ${ }^{3}$ Department of Clinical Hematology, Hospital del Mar, Barcelona, Spain.

Background: Different studies of secondary genetic alterations in diffuse large B-cell lymphomas (DLBCL) have been performed in the pre-rituximab era describing different alterations associated with poor prognosis such as gains of chromosome 3 and 18q. However, no studies are available for patients receiving rituximab-containing regimens.

Purpose of the study: To determine the clinicopathologic significance and prognostic value of chromosomal imbalances in patients with DLBCL treated with rituximab-containing regimens.

Results: We have examined the specimens of 62 patients $(34 \mathrm{M} / 28 \mathrm{~F}$; median age 61 years; range 21-91) diagnosed with DLBCL between 2002 and 2007 using comparative genomic hybridization and immunohistochemistry. All patients received rituximab-containing regimens (in most cases R-CHOP), with $66 \%$ of them achieving CR. CD10, BCL-6, MUM-1 and BCL-2 were used for the immunohistochemical study. Chromosomal imbalances gains $(n=$ $143)$ losses $(n=92)$ and amplifications $(n=6)$ were detected in fiftyfour $(86 \%)$ cases. The most recurrent alterations were gains of $7 q$ (22\%), $12(19 \%), 18 q(19 \%), 1 q(13 \%), 3 q(13 \%)$, and losses of $6 q$ $(22 \%), 1 \mathrm{p}(13 \%)$, and $17 \mathrm{p}(13 \%)$. After a median follow-up of 3.1 years, 20 patients have died, with a median overall survival of 4.5 years. Recurrent high-level DNA amplifications were detected in 18q. Tumors with $6 \mathrm{q}$ losses and high number of gains $(>2)$ were significantly associated with poor prognosis. Neither BCL-2 expression nor the groups defined by immunophenotyping (GC vs non-GC) had prognostic significance in this series.

Conclusion: These results suggest that treatment with rituximabcontaining regimens may modify the prognostic significance of chromosomal imbalances. However, additional studies are needed to assess these observations in larger series of patients.

\section{LS 175}

\section{EXPRESSION OF ANGIOGENIC GROWTH FACTOR RECEPTORS BY NEOPLASTIC CELLS OF CLASSICAL HODGKIN LYMPHOMA}

Aliste C, *Fraga M, Tojo M, ${ }^{1}$ García JF, ${ }^{1}$ Piris MA and Forteza J, for the Spanish Hodgkin Lymphoma Study Group

Complejo Hospitalario Universitario, Santiago de Compostela, ${ }^{1}$ Centro Nacional de Investigaciones Oncológicas, Madrid, Spain.

Background. The availability of antiangiogenic drugs has raised considerable interest in studying the expression of angiogenic molecules in different types of malignancies. In Hodgkin lymphoma (HL), however, only few studies have been performed in small series to address this issue. According to their results, Hodgkin and ReedSternberg (HRS) cells may express angiogenic growth factors and their receptors. Interestingly, VEGF and VEGFR2 co-expression has been reported, leading to hypothesize that these molecules could provide an autocrine pathway of growth stimulation in HRS cells. Purpose. To study the expression of angiogenic growth factor receptors (AGFR) by HRS cells in a large series of classical HL, and its possible association with the expression of cell cycle and apoptosis regulatory proteins. For this purpose, VEGFR1, VEGFR2 and PDGFR $\alpha$ immunostainings were performed in 238 classical HL biopsies using tissue microarrays previously studied for cell-cycle and apoptosis regulatory proteins.

Results. The expression of AGFR by HRS cells (more than $10 \%$ of HRS cells positive) was very frequent (86 to $97 \%$ of the cases). VEGFR1 and VEGFR2 immunostainings were usually cytoplasmic, 
while PDGFR $\alpha$ immunostaining showed also frequent nuclear localization. High expression of the receptors (more than 30\% of HRS cells positive) was found to be significantly associated with the expression of different cell-cycle and apoptosis regulatory molecules: VEGFR1 with MIB1 index and survivin, VEGFR2 with G1/S transition regulators, and PDGFR $\alpha$ with $\mathrm{G} 1 / \mathrm{S}$ and $\mathrm{G} 2 / \mathrm{M}$ transition regulatory proteins, Bcl-XL and MIB1 expression.

Conclusion. HRS cells frequently express AGFR. This is associated with the expression of cell-cycle checkpoints and apoptosis regulatory proteins characteristically altered in HL. These findings suggest that AGFR may play an important role in the proliferation and viability of HRS cells, thus opening new therapeutic pathways for HL.

\section{LS 176}

\section{IMMUNOPHENOTYPIC AND CYTOGENETIC FLUORESCENCE IN SITU HYBRIDIZATION ANALYSIS IN HIGH GRADE B-CELL LYMPHOMAS WITH BURKITT- LIKE FEATURES AND BURKITT LYMPHOMAS}

\author{
Merih Tepeoglu, *Nalan Akyurek
}

Department of Pathology, Gazi University Medical School, Ankara, TURKEY

Background: Diffuse large B-cell lymphoma (DLBCL) and Burkitt lymphoma (BL) are mature aggressive B-cell lymphomas that have heterogeneous clinical, morphological, immunophenotypical and cytogenetic features, and variable clinical course.

Design: We analyzed immunophenotypic and molecular features of 56 lymphomas obtained from patients with typical BL, atypical Burkitt/ Burkitt-like lymphoma (ABL/BLL) and DLBCL. Immunohistochemical staining was performed for CD10, BCL6, BCL2, MUM1 and Ki67. FISH was performed for BCL2 and C-MYC rearrangements, CMYC amplification and PTEN deletion.

Results: In 10 typical BLs, all cases showed an identical immunophenotype (CD10+, BCL6+, BCL2-, and Ki67>99\%), C-MYC rearrangement and no BCL2 rearrangement. C-MYC was amplified in $8(80 \%)$ cases. In $8 \mathrm{ABL} / \mathrm{BLL}$ cases, $8(100 \%)$ were $\mathrm{CD} 10+$, $8(100 \%)$ were BCL6+, $4(50 \%)$ were BCL2+ and $3(37 \%)$ were MUM1+. Ki67 proliferation index was $\sim 88 \%$. C-MYC was rearranged in $6(75 \%)$, BCL2 was rearranged $2(26 \%)$ and C-MYC was amplified in $2(25 \%)$ cases. In 38 DBBHL cases, $12(32 \%)$ were ABC and $26(68 \%)$ were GCB subtype. In eight cases with high Ki67 $(>80 \%)$, C-MYC was rearranged in $4(50 \%)$, BCL2 was rearranged 2 $(25 \%)$ and C-MYC amplified in $5(62 \%)$ cases. C-MYC amplification was found in 21 of 38 (31\%) DBBCL cases. High Ki67 proliferation index did not correlate with morphology, MYC amplification or survival. PTEN deletion was identified in 10 of 38 (26\%) DLBCL, 1 of $10(10 \%) \mathrm{BL}$ and 1 of $8(12 \%) \mathrm{ABL} / \mathrm{BLL}$.

Conclusions: The application of an immunohistochemical panel including markers of germinal-center and non-germinal-center $\mathrm{B}$ cells in combination with FISH analysis for MYC and BCL2 rearrangements might be used to distinguish BL from DLBCL in routine practice. However, many of the high grade B-cell lymphomas are phenotypically and cytogenetically heterogeneous and most of them are found in a spectrum between BL and DLBCL.
LS 177

\section{TCL1A EXPRESSION DELINEATES BIOLOGICAL AND CLINICAL VARIABILITY IN B-CELL LYMPHOMA}

Mohit Aggarwal1, Raquel Villuendas1, Gonzalo Gomez3, Socorro M Rodriguez-Pina1, Margarita Sanchez-Beato1, David Alvarez1, Nerea Martínez1, Antonia Rodriguez1, Maria E Castillo1, Francisca I. Camacho1, Jose A. Garcia-Marco2, Eva Kimby4, David G. Pisano3, Miguel A. Piris1

1Molecular Pathology Programme, Centro Nacional de Investigaciones Oncológicas (CNIO), Madrid, Spain; 2Haematology Department, Hospital Universitario Puerta de Hierro, Madrid, Spain; 3Bioinformatics Unit, Structural Biology, Spanish National Cancer Centre, Madrid, Spain; 4Division of Hematology, Department of Internal Medicine at Huddinge, Karolinska Institutet, Stockholm, Sweden.

Objectives: To explore the biological and prognostic value of TCL1A oncogene in B-cell non-Hodgkin lymphomas.

Materials: The microarray data (GSE9327) from 187 cases of all the major types of B-cell non-Hodgkin Lymphoma (B-NHL) available in the GEO public repository was studied. In addition, 98 CLL cases were studied for TCL1A expression (CLL-specific 19K Oligonucleotide Microarray from Agilent). Eighty-five patients with MCL were analysed on tissue microaarrays for TCL1 protein expression. All cases were histologically reviewed by a team of pathologists (S.M.R. P. and M.A.P.), in accordance with the WHO criteria.

Results: The assembly of a collection of gene-expression signatures of the major types of B-cell NHL identified increased TCL1 expression in multiple lymphoma types. Specifically, Burkitt Lymphoma cases show a homogeneously strong expression of TCL1, while Diffuse Large B-Cell Lymphoma, Follicular Lymphoma, Mantle Cell Lymphoma (MCL), Chronic Lymphocytic Leukemia (CLL), Nodal Marginal Zone Lymphoma and Splenic Marginal Zone Lymphoma display a striking variability in the intensity of TCL1 staining. This was validated in two independent series. A Gene Set Enrichment Analysis of the genes correlated with TCL1A expression found that variation in the level of expression of TCL1A was significantly associated with some of the most important gene signatures recognizing B-cell lymphoma pathogenesis and heterogeneity, such as Germinal center, B-Cell Receptor, NF-kB (and its target genes), Death, MAP kinases, TNFR1, TOLL, and IL1R. Additionally, TCL1 microarray expression was correlated with shorter time to treatment in CLL cases. In MCL the level of protein expression of TCL1 was correlated with overall survival. These results demonstrate the clinical and biological significance of TCL1 expression.

Conclusions: TCL1A oncogene plays a crucial role in the pathogenesis and heterogeneity observed in B-NHL. Additionally, it has the potential to be a prognostic marker in patients with CLL and MCL. 


\section{LS 178}

\section{GENE EXPRESSION PROFILING REVEALS A DISTINCT MOLECULAR SIGNATURE FOR NASAL NK/T-CELL LYMPHOMAS.}

Yenlin Huang $^{1 *}$, Aurelien de Réynies ${ }^{2}$, Laurence de Leval ${ }^{3}$, Nadine Martin-Garcia ${ }^{1}$, Jacques Bosq ${ }^{4}$, Barbara Petit ${ }^{4}$, Anne-Catherine Baglin $^{4}$, Emilie Thomas ${ }^{2}$, Francois Radvanyi ${ }^{5}$, Paul Coppo ${ }^{6}$, Christian Schmitt $^{1}$, Philippe Gaulard ${ }^{1}$.

${ }^{1}$ Pathology and Inserm U841, CHU Henri Mondor, Créteil, France ; ${ }^{2}$ Ligue Nationale contre le Cancer, Paris, France ; ${ }^{3}$ Pathology, CHU Sart-Tilman, Liège, Belgium ; ${ }^{4}$ Groupe d'étude des lymphomes de l'adulte (GELA), Paris, France; ${ }^{5}$ Institut Curie, Paris, France; ${ }^{6}$ Hematology, Hôpital Saint-Antoine, Paris, France.

Nasal NK-/T-cell lymphomas (NK/T) is a rare disease entity derived from NK or cytotoxic T cells. Besides its association with EBV, the pathogenesis and genetic alterations underlying NK/T remain poorly understood.

The aim of this study was to identify the molecular signature of this rare lymphoma and explore the molecular pathways implicated in its pathogenesis.

Total RNAs of 7 NK/T tumor samples, 2 NK/T tumor-derived cell lines, 2 normal NK cell samples together with 16 peripheral T-cell lymphoma unspecified (PTCL-U) were hybridized on Affymetrix HG-U133A Plus2.0 microarray. In parallel, array-CGH was performed using a DNA microarray comprising $4434 \mathrm{BAC}$ clones with a resolution of $600 \mathrm{~KB}$. Compared to normal NK cells, NK/T was characterized by overexpression of genes involved in cell-cell interaction (integrins, Ecadherin, VCAM1), extracellular matrix (TIMP1, TIMP2, TIMP3), innate immune responses (Toll-like receptors), genes encoding chemokines (CXCL9/Mig, CXCL10/IP-10, CXCL 12/SDF-1), oncogenes (mafb, c-myc,..), growth factors (PDGFR) and EBV-related gene (EBI3). NK/T was further compared to PTCL-U to define its specific molecular signature: it was characterized by overexpression of genes encoding NK-cell associated molecules (CD56, CD244, KIRs,..), cytotoxic proteins (Granzymes B, H, perforin,..), chemokines (CCL5/RANTES, CCL4/MIP-1-beta) and apoptosis-associated molecules such as Fas ligand. By immunohistochemistry, Granzyme H was demonstrated in all NK/T tested $(n=9)$. Apoptosis, AKT and Jak-Stat pathways were among the most differentially expressed pathways and phosphorylated Stat3 (Tyr705) was constitutively expressed in the nuclei of neoplastic cells (9/10 cases). Genomic profiles determined recurrent gains on 1q21-q44, 6p11-p25 and 7q11-q36, and losses on $6 \mathrm{q} 16-\mathrm{q} 25$ and 11q24-q25 and identified candidate genes with correlation with transcriptome.

The current study (1) verifies the cell origin of NK/T from activated NK cells, (2) identifies a distinct molecular signature for NK/T, suggesting involvement of pathways potentially implicated in their pathogenesis and showing correlation with recurrent genomic alterations.
LS 179

\section{IMAGE-GUIDED CORE-NEEDLE BIOPSY (CNB) IN THE MANAGEMENT OF PATIENTS WITH NON- HODGKIN'S LYMPHOMA AND HODGKIN'S DISEASE.}

* C Chassagne-Clément (MD), D Ranchère (MD), P Thiesse (MD), E Callet-Bauchu (MD-PHD), I Tigaud (MD), T Gargi, C Bergeron (MD), P Biron (MD), H Ghesquières (MD) and C Sebban (MD).

Centre Léon Bérard, Lyon, and Centre Hospitalier Lyon Sud, Pierre Bénite - France.

Introduction: $\mathrm{CNB}$ are useful to the tumor diagnosis. We reported our experience in evaluating the efficacy of $\mathrm{CNB}$ in the management of NHL and HD.

Methods: $251 \mathrm{CNB}$ were performed as the diagnostic procedure on 226 patients treated in our institution for a NHL or a HD between 1997 and 2006. This procedure was achieved in 107 cases without previous history of lymphoma, in 139 with suspected recurrence and in 5 as a second procedure after a first inconclusive CNB, and was performed under ultrasound, CT-scan or radioscopy guidance. Specimens were obtained from deep-seated (120) or peripheral lymph nodes (76), limbs (18), chest wall or paravertebral areas (18), head and neck areas (8), breast (7) and bone (4). Standard histology was completed by immunohistochemistry in all cases but 7 . The originality of our serie is the achievement of a genetic study in 54 cases $(9$ as a molecular genetic study on frozen samples and 45 as a cytogenetic analysis on fresh tissue samples).

Results: Diagnosis of lymphoma was achieved in all but one case with no need of surgical biopsy. In 101 patients without previous history of lymphoma, CNB provided sufficient material for the diagnosis. Cytogenetic was informative in 27 cases (conventional analysis in 16 , fluorescent in situ hybridization in 11) and permitted to change the initial diagnosis in 1 case. Treatment was initiated solely on the basis of the diagnosis obtained by the CNB as the first procedure in 245 out of 251 cases.

Conclusion: Our data suggest that $\mathrm{CNB}$ is a suitable and adequate procedure in the diagnosis of lymphomas, particularly for patients with previous history of lymphoma. However at the time of initial diagnosis we recommend the use of CNB only in the absence of peripheral lesions or for patients inappropriate for surgery.

\section{$\underline{\text { LS } 180}$}

\section{NON GERMINAL CENTER PHENOTYPE IS FREQUENTLY ASSOCIATED WITH CHRONIC HEPATITIS C INFECTION IN EGYPT}

Howayda Abd El All*(1) (2) and Racha Metwally (3)

1) Pathology Department, Faculty of Medicine, Suez Canal University, Ismailia

2) Immunohistochemistry Laboratory, Nasser Institute, MOHP, Cairo

3) Oncology Department, Nasser Institute, MOHP, Cairo, Egypt

Background: Hepatitis $\mathrm{C}$ virus (HCV) is a major health problem in Egypt responsible for liver cirrhosis and cancer. The estimated adjusted national prevalence rate of chronic hepatitis $\mathrm{C}$ infection is 
7.8\%. Aim: to assess the phenotype of diffuse large B cell lymphoma (DLBCL) associated with HCV infection in the Egyptian population. Methods: 35 cases diagnosed as DLBCL were first classified into germinal centre (GC) B cell like and non GC origin either activated $\mathrm{B}$ cells $(\mathrm{ABC})$ or type 3 based on their immunoreactivity for CD10, bcl6 and Mum-1. Bcl-2 and MIB-1 as adverse prognostic factors were also evaluated. The phenotype was further correlated with the patients' clinicopathological characteristics. HCV infection was estimated by anti-HCV antibody.

Results: twelve cases (34.3\%) positive for CD10 and/or bcl-6 were classified as GC B cell like subtype, 10 cases positive for Mum-1 as ABC subtype (28.6\%), and 13 cases double positive or negative for bcl-6 and Mum-1 were considered as type 3 (37.1\%). HCV infection was strongly associated with non GC phenotype specially type $3(\mathrm{p}=$ 0.025 for $\mathrm{ABC}$ and $\mathrm{p}<0.001$ for type 3 ). In addition, the expression of bcl-2 and MIB-1 was associated with HCV infection ( $p<0.01$ for both). Extranodal DLBCL were frequently HCV positive and the most common site was the liver $(\mathrm{p}<0.01)$.

Conclusion: DLBCL HCV+ was almost of post GC phenotype specially type 3 , had high proliferation fraction, aggressive phenotype an extranodal presentation.

\section{LS 181}

\section{ECA39/BCAT1, A GERMINAL CENTRE-ASSOCIATED MARKER EXPRESSED IN B-CELL AND T-CELL LYMPHOMAS}

TI George, ${ }^{1}$ E Ballabio, ${ }^{2}$ JC Paterson, ${ }^{2}$ SA Pileri, ${ }^{3}$ M-L Hansmann, ${ }^{4}$ W Klapper, ${ }^{5} \mathrm{~T}$ Longacre, ${ }^{1} \mathrm{RB}$ West, ${ }^{1} \mathrm{M}$ van de Rijn, ${ }^{1} \mathrm{~J}$ Boultwood, ${ }^{2}$ $\mathrm{J}_{\text {Wainscoat }}{ }^{2}$ and $\mathrm{T}$ Marafioti ${ }^{2}$

${ }^{1}$ Department of Pathology, Stanford University School of Medicine, Stanford, California, USA

${ }^{2}$ Leukaemia Research Fund Immunodiagnostics Unit, Nuffield Department of Clinical Laboratory Sciences, John Radcliffe Hospital, University of Oxford, UK

${ }^{3}$ Department of Pathology and Unit of Haematopathology, 'L\&A Seragnoli' Institute of Haematology, Univeristy of Bologna, Bologna, Italy

${ }^{4}$ Senckenberg Pathology Institute, Johann Wolfgang Goethe-University Clinic, Frankfurt am Main, Frankfurt am Main, Germany

${ }^{5}$ Department of Hematopathology and Lymph Node Registry, Kiel, Schleswig-Holstein University Hospitals, Kiel, Germany
ECA39, also known as branched chain aminotransferase 1 (BCAT1), is regulated by $c-M y c$ in humans with increased RNA expression found in Burkitt lymphoma. We document ECA39/BCAT1 expression at the protein level in human tissues by immunohistochemistry and western blotting analysis using an antibody reactive with paraffinembedded tissues. ECA39/BCAT1 was highly expressed in germinal centre B-cells, in monocytoid B-cells, in splenic venous endothelium and in CD34-positive marrow blasts. It was absent in immature and mature thymocytes, with only a few scattered positive cells coexpressing $\mathrm{CD} 20$ in the cortical medulla. Rare follicular $\mathrm{T}$ cells also coexpressed ECA39/BCAT1. Among lymphoid neoplasms, ECA39 was found in Burkitt lymphoma, diffuse large B-cell lymphoma, classical Hodgkin lymphoma and anaplastic large cell lymphoma. Expression in follicular lymphoma was heterogeneous with greater expression seen in higher grade tumors. Approximately one-half of chronic lymphocytic leukaemias were positive with strong staining observed in larger lymphocytes while small lymphocytes showed weak to negative staining. Expression was generally not seen in mantle cell lymphoma, hairy cell leukaemia, lymphoplasmacytic lymphoma and myeloma/plasmacytoma. Precursor B and $\mathrm{T}$ cell lymphoblastic lymphoma/leukaemia, peripheral T-cell lymphoma, angioimmunoblastic T-cell lymphoma, lymphocyte predominant Hodgkin lymphoma, and acute myelogenous leukaemia also showed expression in a subset of cases. In non-haematopoietic neoplasms, ECA39 was present in testicular embryonal carcinoma and ovarian sex cord stromal tumours, with expression seen in a subset of cases of colon carcinoma, ovarian endometrioid carcinoma, and endometrial carcinoma of the uterus. ECA39 shows an expression pattern similar to that of Jaw1/LRMP, HGAL and PAG in human B-lymphoid malignancies; in T-lymphoid malignancies, ECA39 expression is similar to that of PAG. The expression of ECA39/BCAT1 may be of interest in the differential diagnosis and research of haematolymphoid and non-haematopoietic tumours neoplasms. 


\begin{tabular}{|c|c|c|c|c|c|}
\hline Abad, M. & LS 110 & Bağır, E. & LS 104 & Bonetti, F. & LS 50 \\
\hline Abd El All, H. & LS 180 & Baglin, A. C. & LS 178 & Bonino, L. D. & BMS 6 \\
\hline Abraham, E. & BMS 26 & Bagnara, G. P. & LS 54 & Boniver, J. & LS 172 \\
\hline Abruzzo, L. V. & LS 49 & Bahler, D. W. & LS 49 & Booman, M. & LS 98 \\
\hline Acevedo, A. & BMS 15, LS 61 & Baia, M. & LS 161 & Bordessoule, D. & LS 130, LS 131, LS 165 \\
\hline Achille, P. & BMS 27 & Balagué, O. & LS 39 & Borisch, B. & LS 27 \\
\hline Adam, P. & LS 40, LS 74 & Balhárek, T. & LS 42, LS 126, LS 154 & Borrebaeck, C. & LS 26 \\
\hline Agan, M. & LS 97 & Ballabio, E. & LS 72, LS 181 & Bosari, S. & BMS 22 \\
\hline Aggarwal, M. & LS 177 & Balogh, Z. & LS 8, LS 111 & Bosch, F. & LS 18 \\
\hline \multirow[t]{2}{*}{ Agostinelli, C. } & BMS 15, LS 15, & Banerjee, D. & LS 25 & Boskovic, D. & BMS 23, LS 173 \\
\hline & LS 18, LS 54 & Barbi, S. & LS 22, LS 152, LS 168 & Bosq, J. & LS 178 \\
\hline Ajdukovic, R. & LS 113 & Barrionuevo, $\mathrm{C}$. & LS 110 & Bossard, C. & LS 107 \\
\hline Akalin, T. & LS 160 & Barry, T. & LS 133 & Bossi, F. & BMS 10 \\
\hline Akkaya, B. K. & LS 29 & Barthova, M. & LS 42, LS 126, LS 154 & Bot, F. & BMS 29 \\
\hline Akyurek, N. & LS 176 & Basic-Kinda, S. & LS 113 & Bouabdallah, R. & LS 108, LS 129 \\
\hline Algara, $\mathrm{P}$. & LS 56 & Basrur, V. & LS 80 & Boudjemaa, S. & LS 77 \\
\hline Aliste, $\mathrm{C}$. & LS 175 & Bassarova, A. & LS 69 & Boufama, M. & LS 102 \\
\hline Alkan, G. & LS 29 & Basta-Jovanović, & BMS 7 & Boultwood, J. & LS 181 \\
\hline Allmer, C. & LS 132 & Baudis, M. & LS 141 & Bousquet, M. & LS 1 \\
\hline Alonso, R. & LS 149 & Baumforth, K. R & LS 68 & Bouzid-Bendisari, K. & LS 102 \\
\hline Alvarez, D. & LS 177 & Beà, S. & LS 18, LS 19, LS 43 & Boveri, E. & BMS 5, BMS 13 \\
\hline Amato, E. & LS 152, LS 168 & & LS 44, LS 174 & Bower, M. & LS 125, LS 134 \\
\hline Ambrogio, C. & LS 163 & Beaugerie, L. & LS 156 & Brais, R. & LS 158 \\
\hline Anagnostopoulos, A. & BMS 16, BMS 19 & Belbachir, A. & LS 115 & Bräuninger, A. & LS 14, LS 41 \\
\hline Anastasopoulou, A. & LS 166 & Belessi, C. & BMS 14 & Braza, M. & LS 67 \\
\hline Androulaki, A. & BMS 32, LS 166 & Bellan, C. & S 70, LS 78, LS 79, LS 118 & Braziel, R. & LS 24, LS 43, LS 44 \\
\hline Angeles-Angeles, A. & LS 164 & Bellas, C. & LS 56, LS 114 & Briere, J. & LS 161 \\
\hline \multirow[t]{2}{*}{ Angelopoulou, M. K. } & BMS 32, LS 153, & Benayad, S. & LS 117, LS 150 & Broccardo, C. & LS 1 \\
\hline & LS 166 & Benedetti, D. & BMS 10 & Bronz, L. & LS 118 \\
\hline Annunziato, F. & LS 168 & Benhamou, E. M & LS 148 & Brousse, N. & LS 142, LS 156 \\
\hline Anthony, A. & LS 169 & Benkirane, A. & LS 115, LS 117, LS 120, & Brousset, P. & LS 1 \\
\hline Antonicelli, G. & LS 71 & & LS 121, LS 150 & Bruneau, J. & LS 142 \\
\hline Arai, E. & LS 90 & Bentink, S. & LS 116 & Bueso-Ramos, C. E. & LS 147 \\
\hline Aralica, M. & BMS 31 & Berger, F. & LS 161 & Buglioni, S. & LS 145, LS 146 \\
\hline Arbor, A. & LS 133 & Bergeron, $\mathrm{C}$. & LS 179 & Bulian, P. & LS 31 \\
\hline Arcaini, L. & BMS 5 & Bernardo, A. D. & BMS 13 & Bumbasirevic, V. & LS 173 \\
\hline Arenillas, L. & LS 174 & Bertolaso, A. & LS 106, LS 152, LS 168 & Burger, J. A. & BMS 28 \\
\hline Arzu Saglam, E. & LS 28 & Bertucci, F. & LS 129 & Burjanivova, $\mathrm{T}$. & LS 126 \\
\hline Asami, E. & LS 90 & Bessie, B. & LS 78 & Burroni, B. & BMS 5 \\
\hline Asnafi, V. & LS 142 & Bianchi, P. & BMS 22 & Butsch, R. & LS 82 \\
\hline Asplund, C. & LS 162 & Bianchi, E. & LS 22, LS 36 & & \\
\hline Assaf, C. & LS 148 & Billings, $\mathrm{T}$. & LS 23 & Caggiari, L. & LS 31, LS 33 \\
\hline Atayar, C. & LS 94, LS 137 & Bink, K. & LS 167 & Cagirgan, $\mathrm{S}$. & LS 144 \\
\hline \multirow[t]{2}{*}{ Athanasiadou, I. } & BMS 16, BMS 18 , & Birgersdotter, A. & LS 68 & Cairo, M. S. & LS 80 \\
\hline & BMS 19 & Biron, P. & LS 179 & Callet-Bauchu, E. & LS 179 \\
\hline Atsaves, V. & LS 96 & Bisig, B. & LS 172 & Camacho, F. I. & LS 177 \\
\hline Attygalle, A. D. & LS 122 & Björkholm, M. & LS 68 & Camarero, C. & LS 53 \\
\hline Auer, I. & BMS 20, BMS 30 & Blokzijl, T. & LS 93, LS 94, LS 98 & Campidelli, C. & BMS 15, LS 13 \\
\hline Aumueller, C. & BMS 8 & Bloom, K. & LS 169 & Campo, E. BMS 15 & $15, \operatorname{LS} 7, \operatorname{LS} 18, \operatorname{LS} 19$, \\
\hline Aurer, I. & LS 113 & Bödör, C. & LS 8, LS 111 & LS 39, LS 43, L & LS 44, LS 100, LS 149, \\
\hline Avet-Loiseau, H. & LS 107 & Boerma, E.-J. & LS 99, LS 139 & & LS 174, \\
\hline \multirow[t]{2}{*}{ Aymerich, M. } & LS 18 & Boerma, E. G. & LS 141 & Canales, M. & LS 60 \\
\hline & & Bogdanovic, A. & LS 173 & Canalini, P. & LS 146 \\
\hline Bacon, C. M. & LS 40, LS 158, LS 159 & Boiocchi, L. & BMS 22 & Cañamero, $\mathrm{M}$. & LS 157 \\
\hline Baena, S. & LS 157 & Bomben, R. & BMS 10 & Cangi, M. G. & LS 50 \\
\hline
\end{tabular}


Canioni, D.

Cannizzaro, R.

Cánovas, A.

Cantisani, R.

Canzonieri, V. LS 31, LS 32, LS 33, LS 34

Cardesa, T.

Carreras, J.

Carro, A.

Carugi, A.

Casanova, L.

Casasnovas, O.

Casavilca, S.

Castello, A.

Castillo, I.

Castillo, M. E.

Castro, Y.

Catherwood, M. A.

Cazzola, M.

Cecconi, D.

Čemerikić-Martinović, V. BMS 7, BMS 23,

LS 173

LS 123

LS 132

Cerf-Bensussan, N.

Cerhan, J.

Cerino, G.

Čermák, M.

Chablé-Montero, F.

Chadburn, A.

Chan, W.-C.

Chanock, S.

Chanudet, E.

Chapman, J.

Chassagne-Clément, C.

Chen, D.

Chen, H.-K.

Chen, S.

Cherry, D.

Chetaille, B.

Chiarle, R.

Chilosi, M.

Chiodera, C.

Chisesi, T.

Chiu, A.

Chiu, L. L.

Chott, A.

Christensson, B.

Chuah, C. T. H.

Chuang, S.-S.

Cigudosa, J. C.

Claret, F.X.

LS 7, LS 123, LS 158

LS 37, LS 55

LS 70, LS 71

LS 110

LS 129

LS 110

BMS 5

LS 56

LS 57, LS 177

LS 57

LS 48

BMS 5

LS 22, LS 36

Cocco, M.

LS 70, LS 71, LS 78, LS 79,

LS 118

Coggi, G.

Coiffier, B.

Colarossi§, C.

Colleoni, G. W. B.

LS 17, LS 79

LS 42

LS 164

BMS 21

LS 2, LS 43, LS 44

LS 132

LS 122

BMS 11

LS 179

LS 47

LS 4

LS 133, LS 169

LS 89

LS 108, LS 129

LS 163

LS 22, LS 36, LS 106

LS 38

LS 15

BMS 21

LS 9

LS 14, LS 72

LS 119, LS 162

LS 10

LS 3, LS 4, LS 6, LS 123

LS 37, LS 55, LS 57

LS 96

Colomer, D. LS 18, LS 19, LS 100, LS 149

Colomo, L.

LS 7, LS 39, LS 174

Concepción, R.

Conde, E.

Conlon, K. P.

Connors, J. M.

LS 80

LS 63
Conti, S.

Copie-Bergman, C.

Copie, C.

Copin, M. C.

Coppo, P.

Cordone, I.

Corine, J.

Corinne, $\mathrm{H}$.

Cornillet-Lefebvre, P.

Corti, D.

Costa, C.

Costanzo, V.

Coste-Martineau, V.

Coste, V.

Costes, V.

Cotta, C. V.

Coyaud, E.

Cozen, W.

Cristóbal, E.

Croce, $\mathrm{C}$.

Cruz, $\mathrm{M}$.

Csernus, B.

Csomor, J.

Cuillière-Dartigues, $\mathrm{P}$.

Currie, $\mathrm{T}$.

D'Amore, F.

D'Andrea, M.

Dal Bo, M.

Dal Cin, E.

Dastugue, N.

de Dios Soler, M.

De Falco, G. LS 17, LS 70, LS 71, LS 78 ,

de Jong, D.

de Leval, L.

de Mascarel, A.

de Nully Brown, P.

de Prenter, M.

de Raeve, $\mathrm{H}$.

De Re, V.

de Réynies, A.

Deaglio, S.

Deák, L.

Degan, M.

Del Poeta, G.

Delabesse, E.

Deliliers, G. L.

Della Porta, M. G.

Delsol, G.

Desouki, M. M.

Dessanti, L.

di Celle Paola, F.

Di Lisio, L.

Di Raimondo, F.

Diaz de Otazu, R.

Dictor, M.

Dimosthenous, K.

Ding, $\mathrm{H}$.

Dirnhofer, S.

Dobrea, C.

LS 146

LS 161

LS 77

LS 129

LS 178

LS 146

LS 73

LS 161

LS 148

LS 118

LS 163

LS 79

LS 131

LS 165

LS 67

BMS 11, LS 91

LS 1

LS 132

LS 127, LS 128

LS 152, LS 155, LS 168

LS 60

LS 8

LS 8, LS 111

LS 156

LS 76

LS 15

LS 146

BMS 10

LS 50

LS 1

LS 135

LS 79

LS 98

LS 122, LS 172, LS 178

LS 131

LS 66

LS 20, LS 73

BMS 29

LS 31, LS 32, LS 33, LS 34

LS 178

BMS 10

LS 8, LS 111

BMS 10

BMS 10

LS 1

BMS 22

BMS 5

LS 1

LS 89

LS 146

BMS 27

LS 37, LS 55, LS 58

LS 15

LS 56

LS 26

BMS 18

LS 27

Dogan, A. LS 35, LS 47, LS 123, LS 132,

LS 97

Doglioni, C.

LS 50

Doig, T. N.

LS 151

Dolcetti, R.

LS 50

Domínguez-Franco, P. LS 61

Dotlic, S. LS 113

Doussis-Anagnostopoulou, I. LS 153

Drakos, E. LS 62, LS 91, LS 96

Drakos, I.

LS 147

Dreyling, M.

LS 64

Du, M.-Q. LS 40, LS 122, LS 123, LS 158,

LS 159

$\begin{array}{ll}\text { Duchez, S. } & \text { LS } 130 \\ \text { Dukova, B. } & \text { LS } 171\end{array}$

Dunbar, D. LS 151

Dündar, E. LS 105

Duval, A. LS 156

Ehlers, A.

LS 116

Eickhoff, J. C.

LS 2

Ek, S.

LS 26

El Sheikh, S.

Elenitoba-Johnson, K. S. J.

LS 5

Emara, K. A.

LS 80

Erber, W.

BMS 2

Ergin, M.

LS 109

LS 104

Erickson, L. A.

LS 35

Eriksson, M.

LS 119

Ernberg, I.

Espinet, B.

Esposito, A.

Estelle, Ch.

Esterni, B.

LS 68

BMS 15, LS 18

LS 54

Fabiani, B.

Fabrizio, T.

Facchetti, F. LS 38, LS 50, LS 84, LS 86

Facciolo, F.

Fadlelmola, F.

LS 145

Fameli, M.

LS 25

Familiades, J.

BMS 16

Fan, G.

LS 1

Farinha, P.

LS 24

Fassas, A. 
Feuillard, J.

Filipovski, V.

Finetti, P.

Finn, W. J.

Fisogni, S.

Fléjou, J. F.

Florena, A. M.

Flygare, J.

Ford, R. J.

Forestier, L.

Forteza, J.

Fosså, A.

Fraga, M.

Francesca, R.

Francesca, S.

Francesco, A.

Francia di Celle, P.

Franco, V.

Fresno, M. F.

LS 130, LS 131, LS 165

LS 171

LS 129

LS 133

LS 38

LS 156

BMS 13

LS 119, LS 162

LS 62

LS 130

LS 175

LS 69

LS 175

LS 54

BMS 27

LS 54

BMS 6

BMS 10, BMS 13

LS 53

Gachard, N.

Gagyi, É.

Gaidano, G.

Gaillard, F.

Galani, Z.

Gallamini, A.

Gallo, E.

Gao, Z.

García-Cosío, M.

García-Laraña, J.

Garcia-Marco, J. A.

García, J. F.

Gargi, T.

Gariphalia, K.

Gascoyne, R. D.

Gattei, V.

Gatter, K.

Gatter, K. M.

Gaulard, P.

Gazzola, A.

George, T. I.

Georgiadi, E.

Gernert, C.

Gesk, S.

Ghaleb, F. M.

Gheorghe, G.

Ghesquières, $\mathrm{H}$.

Gianelli, U.

Gibcus, J.

Giefing, M.

Giger, S.

Giordano, A.

Gjerdrum, L. M.

Godio, L.

Gofflot, S.

Gomez, G.

Gomez-Abad, C. I.

Gomez-Lozano, N.

Gómez, $\mathrm{H}$.

Gong, L.

Gönlüșen, G.

LS 107

LS 166

LS 15

LS 145

LS 112

LS 127, LS 128

LS 127, LS 128

LS 177

LS 52, LS 53, LS 175

LS 179

BMS 17, BMS 18

LS 43, LS 44, LS 63

BMS 10, LS 31

LS 51

LS 24

LS 72, LS 161, LS 178

LS 54

BMS 4, LS 181

LS 153

LS 74

LS 14

BMS 2

BMS 21

LS 179

BMS 13, BMS 22

LS 93

LS 14

LS 83, LS 85

LS 70

LS 66, LS 87

BMS 6

LS 172

LS 37, LS 55, LS 177

LS 37, LS 55

LS 114

LS 110

LS 112

LS 104
Gonzalez-Medina, A. R.

Goodlad, J. R.

Gorgoulis, V.

Gotic, M.

Goval, J. J.

Grados, O.

Grande, C.

Grange, F.

Grant, J. W.

Gratwohl, A.

Graziani, D.

Green, T. M.

Gregory, C.

Greiner, T. C.

Griskevicius, L.

Groenen, P. J. T. A. LS 20, LS 21, LS 73,

Grogg, K. L.

Guarnotta, C.

Guillaume, Y.

Guinebretière, J. M.

Gulbas, Z.

Gunn, S. R.

Gustafsson, K.
Habermann, T.

Hager, I.

Haholu, A.

Hamoudi, R.

Hamoudi, R. A. LS 7, LS 40, LS 123, LS 159

Han, X.

Hansmann Martin, L.

Hansmann, M.-L.

Hanson, C. A.

Haralambieva, E.

Harald, S.

\author{
E.
}

Harms, G.

LS 93 , LS 94, LS 98, LS 99

Hartge, $P$.

LS 93, LS 94, LS 98, LS 99

Hartmann, E.

Hartmann, E. M.

LS 132

Hartmann, E. M.

Hartmut, B.

Hartmut, M.

Hasserjian, R. P.

, LS 54

BMS 28

LS 143

LS 133

Hazar, V.

LS 29

Hazem, A.H.I. LS 134, LS 124, LS 125

Hebeda, K. BMS 29, LS 20, LS 73, LS 75,

Hebeda, K. M.

LS 103

Heegaard, S.

Heinz-Wolfram, B.

Hekimgil, M.

Hentschel, U.

Hernández, L.

Herreros, B.

Hibbard, M.

Hii, A.

Hirose, $\mathrm{T}$.

Hirsch, E.

Hirschmann, P.

Hoefig, K. P.

LS 138, LS 143

LS 144, LS 160

LS 74

LS 19, LS 39

LS 56

LS 169

LS 169

LS 90

LS 163

LS 46

LS 143
LS 22, LS 43

Hoessli, D. C.

LS 27

Hoevenaars, B.

LS 103

Höller, S. LS 45, LS 46, LS 59, LS 64

Horwitz, J.

BMS 11

Howat, W.

Hsieh, P.-P.

LS 7

Huang, J. Z.

LS 3, LS 4

LS 24

Huang, W.-T.

LS 3, LS 4

Huang, $X$.

LS 112

Huang, Y. LS 112, LS 122, LS 158, LS 159

LS 178

Hull, D.

LS 48

Hummel, M.

Hüsken, J.

LS 116, LS 155

LS 14

Hutter, G.

LS 64

Ib Jarle, C.

Ibrahim, S.

LS 66

BMS 2

LS 40

Ichimura, $\mathrm{K}$.

LS 69

Ida Munster, I.

LS 42

Ilenčíková, D.

BMS 9

Ilham, Y.

LS 113

Iman, M. M.

BMS 9

Iman, $\mathrm{T}$.

LS 5

Imperatore, F. LS 17

Incardona, P. LS 38

Incili, N. LS 144

Inga, $\mathrm{N}$.

LS 14

Inghirami, G. LS 163

Ingrao, S. BMS 13

Invernizzi, R. BMS 5

Ionescu, B. LS 140

Iriso, R. LS 79

Isaacson, P. G. LS 4, LS 40, LS 122, LS 123,

LS 158

Ishizawa, $\mathrm{K}$

LS 90

Isiksoy, S. LS 105

Iurlo, A.

BMS 22

Jabri, L. LS 115, LS 117, LS 120, LS 121,

Jaffe, E. S.

LS 150

Jaković, L.

LS 43, LS 44

Jakovic, L. J.

BMS 7, LS 173

BMS 23

Jan-Lukas, R.

LS 98, LS 99

Jan, D.

Janevska, V.

LS 43, LS 44, LS 69

LS 171

LS 20

LS 92 Jansen, P. LS 148

LS 43

LS 18

LS 57

LS 58

LS 161

LS 161

Jean-François, E.

Jean-Philippe, J.

Jean-Philippe, M.

Jeffrey Medeiros, L.

LS 14, LS 131 
Jöhrens, K.

Jones, D.

Jones, M.

Jonjić, N.

Jovanovic, M. P.

Jovanovic, R.

Julien, R.

Juskevicius, R.

Juszczynski, P.

Kalagiakou, E.

Kalla, J.

Kamper, P.

Kandiloglu, G.

Kanellis, G.

Karakosta, A.

Karayannis, M.

Karkouri, M.

Katzenberger, T.

Kavantzas, N.

Kayano, H.

Kaygusuz, G.

Kelemen, K.

Kew, A.

Kholousy, N.

Khorshed, A. M.

Kimby, E.

Kinney, M.

Kirchhausen, T.

Kittas, C.

Klapper, W.

Kloboves Prevodnik, V.

Kluin, M.

Kluin, $\mathrm{P}$.

Kluin, P. M.

Ko, Y.-H.

Koay, E. S. C.

Koch, I.

Koerbel, L.

Koković, I.

Koopmans, S.

Kopper, L.

Korkolopoulou, P.

Korol, D.

Krampera, M.

Kremer, M.

Kroesen, B. J.

Ksenija, L.

Küppers, R.

Kurrer, M.

Kurtin, P. J.

Kutok, J. L.

Kuzu, I.

Kviatkovská, Z.

Kyoung-Mee, K.

Kyrtsonis, M. C.

Labar, B.

Labrousse, F.

Laforet, M. P.

Lam, K.
LS 155 Langerak, A. W.

LS 96 Lanzavecchia, A.

LS 109 Laoutaris, N.

BMS 31 Lara, C.

BMS 7, LS 173 Lasabova, Z.

LS 171 Laura, G.

LS 130 Laurent, C.

BMS 24 Lavergne-Slove, A.

LS 76 Lavrenčak, J.

Law, K.

BMS 14 Law, M. E.

LS 45, LS 64 Lazarchick, J.

LS 15 Lazzarino, M.

LS 160 Lazzi, S.

BMS 17 Leclair, F.

LS 65 Lee, A. K. W.

BMS 14 Lee, B.

LS 117, LS 150 Lee, E.-S.

LS 45, LS 64 Legrenzi, L.

BMS 32 Leich, E.

LS 90 Lekakis, L.

LS 97 Lenze, D.

LS 24

BMS 24

BMS 2

BMS 2

Leoncini, L.

Leroy, K.

Lesley, C.

LS 119, LS 177 Leucci, E.

LS 133 Leventaki, V.

LS 163 Levi, E.

LS 153 Levidou, G.

LS 14, LS 143, LS 181 Levis, A.

LS 101 Levy, R.

LS 141 Lhermitte, L.

LS 98, LS 99, LS 137

Li, J.

LS 139 Li, M.

LS 30 Liakou, Ch.

LS 9, LS 10 Lian-Hua, K.

BMS 8 Liang, M.

LS 18 Liao, Y.-L.

LS 101 Lilakos, K.

BMS 29 Lim, M.

LS 111 Lim, S. T.

BMS 32, LS 166 Lin, F.

LS 82, LS 83, LS 85

LS 152, LS 168

BMS 8, BMS 25, LS 167

LS 93

BMS 31

LS 14, LS 93

LS 82, LS 83, LS 85

LS 35, LS 47

LS 76

LS 97, LS 105

LS 42, LS 154

LS 30

BMS 32, LS 153

LS 113

LS 131

LS 165

BMS 29
Lin, $\mathrm{O}$.

Lister, A.

Liu, C.

Liu, C. G.

Liu, $\mathrm{H}$.

LS 4, LS 122, LS 123, LS 158 ,

Liu, Z.

Liwski, R.

Lloveras, N.

Loeffler, M.

Lohr, A.

Lome-Maldonado, C.

Longacre, $\mathrm{T}$.

López-Guerra, M.

López-Guillermo, A.

Lopez, A.

LS 100, LS 149

LS 19, LS 39, LS 174
Lonardi, S.
LS 116, LS 155

LS 21 Lorenzo, L.

LS 118 Lorenzon, D.

BMS 14, LS 65

LS 164

LS 126

BMS 27

LS 131

LS 123, LS 158

LS 101

LS 76

LS 35, LS 47

LS 89

BMS 5

LS 78, LS 79, LS 118

LS 107

LS 9

LS 170

LS 11

LS 38

LS 44

LS 96

Lossos, I.

Lossos, I. S.

Lozano, M.

Lucas, S.

Lucioni, M.

Ludovica, R.

Luebbert, M.

Luider, J.

Luigi, T. P.

Luigi, Z. P.

Luzzi, A.

Lydataki, E.

Lynch, C.

M'Hidi, H.

Ma, Y.

Macak, J.

Macintyre, E.

Macon, W. R.

Madani, A.

Mäder, U.

Madsen, J.

Maestre, L.

Magrini, U.

Malavasi, F.

Malcovati, L.

Malpeli, G.

Malter, J. S.

Manazza, A. D.

Mancini, F.

Mangubat, N.

Manioudaki, E.

Mannucci, S.

Mansoor, A.

Mansour, T.

Manucci, S.

Manz, M. G.

Marafioti, T.

Marcinek, J.

Marconi, D.

Marfak, A.

Marijić, B.

Marin, D.

Marino, M.

Marnissi, F.

Marquard, L.

Marquina, B.

Marrella, V.

Martin-Garcia, N.

Martin-Leo H.

Martín-Pérez, D.

Martin-Subero, J. I.

Martin, C. A.

Martin, P.

Martinengo, C.

BMS 15, LS 7

Martínez-Benítez, B

Martinez, A.

Martinez, D.

Martinez, M. A.
LS 78

LS 31

LS 132

LS 136

LS 114

LS 51

BMS 5

BMS 27

BMS 28

BMS 20, BMS 30

LS 54

LS 54

LS 17, LS 70, LS 71

LS 65

LS 132

LS 108

LS 94

LS 64

LS 142

LS 35

LS 115, LS 117, LS 121

LS 45, LS 64

LS 92

LS 58, LS 81, LS 157

BMS 5

BMS 10

BMS 5

LS 152, LS 168

LS 2

LS 163

LS 106

LS 169

BMS 14

LS 71, LS 118

BMS 20, BMS 30

BMS 2

LS 79

LS 118

LS 72, LS 109, LS 181

LS 42, LS 126, LS 154

BMS 10

LS 165

BMS 31

LS 32

LS 145, LS 146

LS 120, LS 121

LS 66

LS 164

LS 86

LS 178

LS 14, LS 16, LS 41

LS 58

LS 14

LS 135

LS 114

LS 163

LS 164

BMS 15, LS 7, LS 39

LS 39

LS 37, LS 55, LS 60 
Martínez, N. $\quad$ LS 37, LS 55, LS 58, LS 60,

Martucci, R.

LS 177

Mas, L.

Masahiro, $\mathrm{H}$.

Mashali, N.

Mason, D.

Mason, D. Y.

Mate, J. L.

Matolcsy, A.

Mattsson, G.

Matutes, E.

Maubec, E.

Maura, R.

Maurer, M.

Maurer, R.

Mazorra, $\mathrm{P}$.

McKenzie, C.

Medeiros, L. J.

Medinger, $\mathrm{M}$.

Meijer, J.

Mekhelef- Itatahine, K.

LS 145, LS 146

LS 110

LS 12

LS 5

LS 109

LS 72

LS 39

LS 8, LS 111, LS 143

LS 109

BMS 15

LS 148

LS 54

LS 132

LS 83

LS 60

LS 170

LS 96, LS 147

BMS 12

LS 103

LS 102

LS 49

Mellink, C. H. M.

Menasce, L.

Menestrina, F.

LS 124, LS 125, LS 134

LS 106

LS 15

LS 119

LS 77

LS 53

LS 56

LS 180

BMS 28

BMS 8

LS 31

BMS 29

LS 88

Mickys,

Mihaljevic, B.

BMS 23, LS 173

Milli, A.

Milpied, P.

Mitrovic, Z.

Moch, $\mathrm{H}$.

Mokhtar, D.

Molina, T.

Molina, T. J.

Mollejo, M.

Mollejo, Ma.

Möller, P.

Montalbán, C.

Montante-Montes, de O. D.

LS 82, LS 83, LS 85

LS 100

LS 113

BMS 9

LS 129

LS 161

LS 60

LS 37, LS 55

LS 93

LS 53, LS 60

LS 164

Montes-Moreno, S. $\quad$ LS 52, LS 56, LS 57 , LS 60, LS 61, LS 81, LS 157

Montes, J.

LS 110

Montes, $\mathrm{S}$.

LS 114

Montresor, M.

LS 106

Montserrat, E. LS 7, LS 100, LS 149, LS 174

Montserrat, E.

LS 18

Moreau, A.

Morente, M. M.

Morettini, F.

Morice, W. G.

Moro, A.

LS 53

LS 17

LS 47

BMS 22
Morris, T. C. M.

Morschhauser, F.

Morton, L.

Mosna, F.

Mozos, A.

Møller, M. B.

Mrhalova, M.

Muenst, S.

Müller-Hermelink, H.-K.

, LS 43,

Murray, P. G.

LS 44, LS 45, LS 64, LS 74

Mwanda, W.

LS 68

LS 79

Načinović-Duletić, A.

BMS 31

Nadarjan, V.

Nagai, $T$.

Nair, R.

Nair, $\mathrm{S}$.

Nam-Cha, S. H.

Nanaji, N.

Narbaitz, M. I.

Naresh, K. N.

Natkunam, Y.

Navarro, A.

Nayak, N.

Nesland, J. M.

Neuberg, D. S.

Nguyen, M.

Nicholson, A. G.

Niculescu Mizil, E.

Nikulshin, S.

Nola, M.

Norton, A. J.

Notarangelo, L. D.

Novaković, S.

Nyagol, J.

LS 99

LS 90

BMS 26

BMS 26

LS 52, LS 61

BMS 21

LS 135

LS 124, LS 125, LS 134

LS 132, LS 136

LS 19

BMS 26

LS 69

LS 76

LS 147

LS 40

LS 140

LS 88

LS 113

LS 44

LS 84, LS 86

LS 101

LS 78, LS 79

O'Malley, D. P.

Oberley, T. D.

Obermann, E. C.

Odum, N.

Oker, E.

Olive, D.

Onnis, A.

LS 70, LS 78, LS 79, LS 155

Orazi, A.

LS 70, LS 78, LS 79, LS 155
BMS 21

Ott, G. BMS 25, LS 43, LS 44, LS 45, LS 64,

Ouyang, J.

LS 74

Ozsan, N

LS 76

Palmer, T.

Pals, S.

Palummo, N.

Panayotidis, $\mathrm{P}$.

Pangalis, G. A.

Pantoula, P.

Papadaki, E.

Papadaki, H.
Papadaki, T.

Papadantonakis, A.
LS 144, LS 160
Papanikolaou, A.

Parasi-Ismailou, A.

Parisi, A.

Park, S.

Parolini, C.

Parrens, M.

Pascual, A.

Pascutto, C.

Pasini, E.

Pasquarella, A.

Passamont, F.

Patel, R.

Paterakis, G.

Paterson, J.

Paterson, J. C.

Patsouris, E.

Patti, C.

Paulli, M.

Pavan, A.

Payne, K.

Pedersen, N. T.

Perea, G.

Pérez Guillermo, M.

Pérez-Galán, P.

Perin, T.

LS 31, LS 32

Perker, M.

Perkins, S. L.

Perrone- Donnorso, R.

Perunicic Jovanovic, M.

Peter Collins, V.

Petersen, J. H.

Peterson, P.

BMS 18

BMS 14, LS 65

LS 22, LS 36

LS 30

LS 36

LS 14, LS 131, LS 165

LS 127, LS 128

BMS 5

LS 50

LS 17

BMS 5

BMS 20, BMS 30

BMS 19

LS 72

LS 181

BMS 32, LS 96, LS 166

LS 15

BMS 5

LS 33, LS 34

LS 158 , LS 159

LS 92

BMS 15

LS 56

Petit, B LS 130, LS 131, LS 165, LS 178

Petrella, T.

Petrusevska, G.

Pfreundschuh, M.

Philip, G.

Philip, W.

Pich, A.

Picquenot, J. M.

BMS 8

LS 133

Pier Paolo, P.

Piero, T.

Piler, S. BMS 15, LS 15, LS 18, LS 54

Pileri, S. A. $\quad$ LS 13, LS 40, LS 46, LS 181

Pillai, N.

LS 109

Piris, M. A. LS 37, LS 52, LS 53, LS 55, LS 57, LS 58, LS 61, LS 81,

LS 175, LS 177

Pisani, F.

LS 145

Pisano, D. G.

Plank, L.

Poliani, L.

Poliani, P. L.

Poncini, D.

Pontikoglou, C.

Ponzoni, M.

Poppema, S.

Porcasi, R.

Porwit, A.

Post, G. R.

LS 37, LS 55, LS 177

LS 42, LS 126, LS 154

LS 50

LS 84, LS 86

LS 83

BMS 19 
Pouliou, E.

Poulsen, C. B.

Prause, J. U.

Prieto, M.

Probst-Hensch, N.

Provencio, M.

Puig, X.

Purdy, K.

Pyrovolaki, K.

Quachouh, M.

Quelen, C.

Quessar, A. LS 115, LS 117, LS 120, LS 121

Quintanilla-Martinez, L.

BMS 8, LS 167

Rabinovich, G. A.

Racanelli, V.

Rachid, M.

Radford, J.

Radman, I.

Radvanyi, F.

Rainey, A.

Ralfkiaer, E.

Ramos, R.

Ranchère, D.

Rashed, R.

Rassidakis, G. Z.

Ravanini, M.

Raymakers, R.

Reijmers, R.

Reiniger, L.

Remstein, E. D.

Renné, C.

Renner, C.

Repsilber, D.

Riemke, J.

Riera, L.

Rigacci, L.

Righi, S.

Rimsza, L. M.

Rinalducci, S.

Rizk, S. H.

Robetorye, R. S.

Robson, A.

Rodig, S. J.

Rodriguez-Pina, S. M.

Rodriguez-Pinilla, S.-M.

Rodriguez, A.

Rodriguez, E.

Rodriguez, $\mathrm{H}$.

Rodriguez, J.

Roehle, A.

Roifman, C. M.

Rombout, P.

LS 76

LS 34

LS 120

LS 40

LS 113

LS 178

LS 51

LS 66, LS 87, LS 92

LS 53, LS 157

LS 179

BMS 2

LS 62, LS 91, LS 96 ,

LS 147

LS 84, LS 86

LS 20, LS 73

LS 93, LS 99

LS 8, LS 111, LS 143

LS 35, LS 47

LS 41

LS 82, LS 83, LS 85

LS 143

LS 14

BMS 6

LS 15

LS 13

LS 43, LS 44

LS 22

BMS 9

LS 49

LS 148

LS 76

LS 177

LS 56, LS 81

LS 177

LS 37, LS 55

LS 60

LS 53

LS 143

LS 84

LS 20, LS 75

Roncador, G. LS 7, LS 61, LS 63, LS 157

Rosati, S. $\quad$ LS 98, LS 99, LS 137, LS 139

Rosenwald, A. BMS 25, LS 18, LS 19,

Rossi, G. LS 22, LS 43, LS 44, LS 45, LS 64

Rossi, J. F.
Rothman, N.

Roué, G.

Rousselet, M. C.

Rousset, T.

Royo, C.

Rozman, M.

Ruiz-Marcellan, M. C.

Rumi, E.

Sachanas, S.

Sachdev, R.

Saez, A.

Safali, M.

Said, J.

Saifi, M.

Salamero, O.

Salar, A.

Salas, C.

Salaverria, I. LS 19, LS 43, LS 44, LS 174

Salcedo, M. T.

Salles, G. A.

Salpeas, V.

Sanchez-Beato, M.

Sánchez-Espiridion, B.

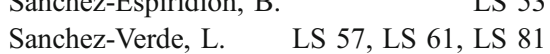

Sánchez, E.

Sanchez, L.

Sander, B.

Sansonno, S.

Santón, A.

Sapia, S.

Sapru, K.

Save, V.

Saydam, G.

Scardoni, M.

Scarpa, A.

Schaerer, S.

Schakel, R. N.

Schlette, E. J.

Schmitt-Graeff, A. H.

Schmitt, C.

Schmitt, S.

Schraders, M.

Schurfeld, K.

Schuuring, Ed.

Sebban, C.

Sehested, M.

Sehn, L.

Seiler, C.

Seili-Bekafigo, I.

Serour, A.

Serrano, S.

Severa, D.

Severson, R.

Shabani-Rad, M.-T.

Shafi, N.

Shi, J.

Shimada, S.

Shipp, M. A.
BMS 32, LS 166

LS 2, LS 37, LS 55,

LS 58, LS 177

LS 132
LS 100, LS 149
LS 107
LS 67
LS 174
BMS 15, LS 18
LS 60
BMS 5

LS 166

BMS 4

LS 60

LS 105

LS 133

LS 67

LS 18

LS 53

LS 114

LS 52

LS 161

LS 58

LS 60

LS 119, LS 162

LS 33, LS 34

LS 127, LS 128

LS 135

BMS 24

LS 158

LS 144

LS 106

LS 106, LS 152, LS 168

LS 82

LS 93

LS 62

BMS 28

LS 178

LS 74

LS 20

LS 79

LS 98, LS 99

LS 179

LS 66

LS 63

LS 80

BMS 31

LS 5

LS 18, LS 53

LS 119

LS 132

BMS 30

BMS 3

BMS 1

LS 90

LS 76

LS 14, LS 141
LS 67 Siebert, R.

Simona, R.

LS 54

Simons, A.

Simula, M. P.

Sismondi, F.

Sista, M. T.

Sjö, L. D.

Sjöberg, J.

Skoda, R.

Slager, S.

Smardova, J.

Smith, C. I. E.

Snyder, M.

Soilleux, E. J.

Solé, F.

Soliman, A. H.

Soubeyran, I.

Soutar, D.

Spasevska, L.

LS 73

LS 32, LS 33

BMS 6

LS 15

LS 92

LS 68

BMS 12

LS 132

LS 64

LS 162

LS 133

LS 51

LS 18

BMS 2

Spina, M.

Spithoven, E.

Stamatopoulos, K.

Stamatoullas, A.

Staudt, L. M.

Stavroyianni, N.

Stelitano, C.

Stephanoudaki, E.

Stevens-Kroef, M.

Stifter, S.

Stöcklein, H.

Stoia, R.

Stucki, V.

Suat-Cheng, P.

Sundram, U. N.

Swerdlow, S.

Szepe, P.

Szepesi, Á.

Tabilio, A.

Talmant, P.

Tamvakos, $\mathrm{H}$.

Tan, L. H. C.

Tan, L.P.

Tao, M.

Tatic, A.

Tauzin, S.

Tedesco, F.

Temmins, C.

Tepeoglu, M.

Ternullo, M. P.

LS 107, LS 131, LS 165

LS 114

LS 171

LS 31

LS 137

BMS 16, BMS 19

LS 129

LS 44

BMS 16, BMS 19

LS 15

BMS 17, BMS 18

LS 73

BMS 31

LS 64

LS 140 
Timár, B.

Timperio, A. M.

Tojo, M.

Tombuloglu, M.

Tondat, F.

Tongson-Ignacio, J.

Tooze, R.

Torchio, $\mathrm{P}$

Tosi, G. M.

Tosi, P.

Traggiai, E.

Trajkovski, Z.

Trassard, M.

Travaglino, E.

Trentin, L.

Tripodo, C.

Troadec, E.

Trøen, G.

Tsao, L.

Tumwine, L. K.

Turner, S.

Tussiwand, R.

Twohig, M.

Tzankov, A.

Tzenou, $\mathrm{T}$.

Uner, A.

Ursuleac, I.

Valdivieso, N.

Valković, $\mathrm{T}$.

van Altena, M. C.

van Cleef, P.

van de Rijn, M.

van den Berg, A.

van den Bosch, C. A.

van Dongen, J. J. M.

van Imhoff, G.

van Krieken, $\mathrm{H}$.
LS 8

LS 36

LS 175

LS 144

LS 163

LS 23

LS 157

LS 15

LS 70

LS 17, LS 118

LS 118

LS 171

LS 77

BMS 5

LS 15

BMS 10, BMS 13

LS 165

LS 69

BMS 11

LS 13

LS 109

LS 118

LS 2

BMS 12, LS 46, LS 59

BMS 32

LS 28, LS 97, LS 105

LS 140

LS 110

BMS 31

LS 75

LS 78

LS 181

LS 93, LS 94, LS 98,

LS 99

LS 71

LS 21

LS 98, LS 99

LS 78 van Krieken, J. H. J. M.

LS 20, LS 21,

LS 73, LS 75, LS 103

Van Marion, A.

BMS 29

van Nierop, S.

van Raaij, A. W. M.

van Rijk, A.

Vassilakopoulos, T.

Vassilakopoulos, T. P.

Vega, F.

Vekhoff, A.

Vela-Chávez, T.

Vener, C.

LS 48, LS 123, LS 158

Vico, E.

Viejo, A.

Villa, A.

LS 139

LS 106

LS 78

BMS 32, LS 166

LS 153

LS 91

LS 129

LS 167

BMS 22

Villamor, N.

Villuendas, R.

LS 146

LS 133, LS 133

LS 84, LS 86

Visser, L.

Vitolo, U.

Voena, C.

Volavšek, M.

Volinia, S.

Vornanen, M.

Vos, H.

Wahab, N. A.

Wainscoat, J.

Wang, $M$.

Wang, S.

Wang, $\mathrm{X}$.

Wartenberg, M.

Watkins, A. J.

Waxman, I.

Weisenburger, D. D.

Wel-Masry, M.

Wendelboe, H. G.

Went, $\mathrm{P}$.

LS 149

LS 15

LS 163

LS 95

LS 155

LS 16

LS 137

BMS 2

LS 181

LS 99, LS 137

LS 119, LS 162

BMS 25

LS 122
Wessendorf, S.

West, R. B.

Willemze, R.

LS 64

Willenbrock, $\mathrm{K}$.

LS 181

LS 148

LS 41

Woetmann, A.

Woodward, J.

LS 87

Wotherspoon, A

Wotherspoon, A. C.

Wright, P.

Xerri, L.

Xyristaki, S.

LS 108, LS 129

BMS 14

Yang, S.-F.

Ye, $\mathrm{H}$.

LS 3

Ye, H. T.

LS 40, LS 158

LS 122

LS 2

Young, A. H.

LS 2

Zafirovski, G.

LS 171

Žákovičová, A.

LS 42

Zamiati, S. LS 115, LS 117, LS 120, LS 121,

LS 150

Zamò, A. LS 22, LS 36, LS 106, LS 152

Zanella, A.

BMS 22

Zanetto, U.

LS 135

Zeng, Q.

LS 158

Zettl, A.

Zevallos-Giampietri, E.

LS 43, LS 44

LS 110

BMS 1

LS 136

LS 23

LS 25

Zhou, M.

Ziepert, M.

LS 80 Zihler, D.

LS 2, LS 43, Zinzani, P. L.

LS 44 Zolla, L.

BMS 9 Zucchetto, A.

LS 109 Zum Bueschenfelde, C. M.

LS 45 Zupo, S.
LS 143

LS 46

LS 15

LS 22, LS 36

BMS 10

BMS 25

LS 152 